RAINFALL OBSERVATIONS USING

DUAL-POLARIZATION RADAR

COUPLED WITH A DROP MOTION

AND EVAPORATION MODEL

\author{
A Thesis \\ presented to
}

the Faculty of the Graduate School

at the University of Missouri-Columbia

In Partial Fulfillment

of the Requirements for the Degree

Master of Science

by

QUINN PALLARDY

Dr. Neil Fox, Thesis Supervisor

DECEMBER 2013 
The undersigned, appointed by the dean of the Graduate School, have examined the thesis entitled

\section{RAINFALL OBSERVATIONS USING \\ DUAL-POLARIZATION RADAR \\ COUPLED WITH A DROP MOTION \\ AND EVAPORATION MODEL}

presented by Quinn Pallardy,

a candidate for the degree of master of science,

and hereby certify that, in their opinion, it is worthy of acceptance.

Professor Neil Fox

Professor Thomas R. Marrero

Professor Anthony Lupo 


\section{ACKNOWLEDGEMENTS}

I would like to thank Dr. Fox, Dr. Marrero, and Dr. Lupo for all the assistance they have given me over the years as both an undergraduate and graduate student.

I also would like to thank Dr. Hubbart for providing much of the rain gauge data, which was essential for the completion of this project. 


\section{TABLE OF CONTENTS}

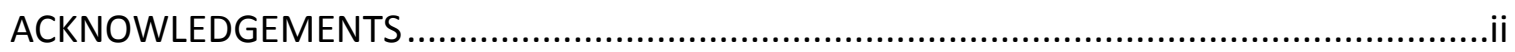

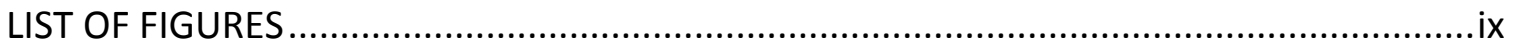

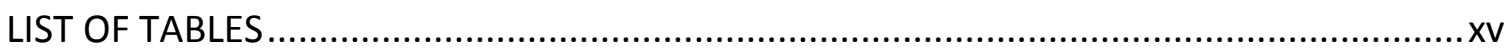

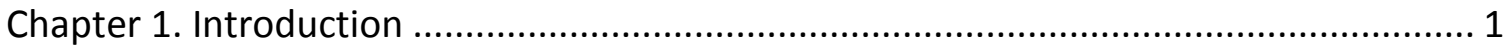

1.1 Importance of Radar Estimation of Rainfall .................................................. 1

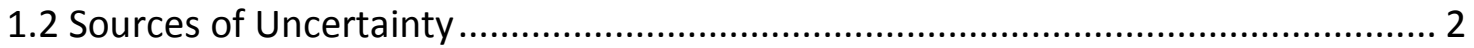

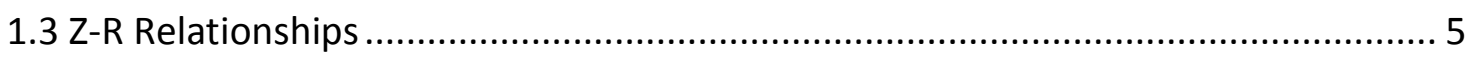

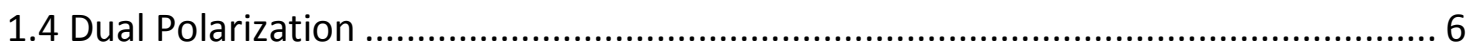

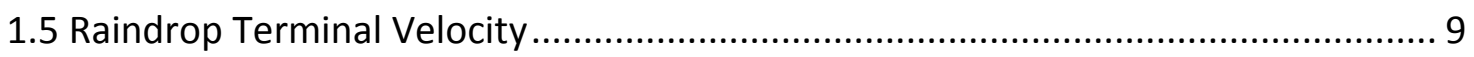

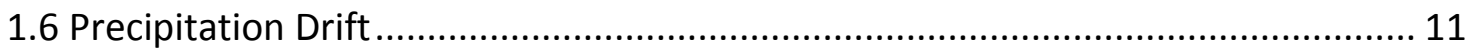

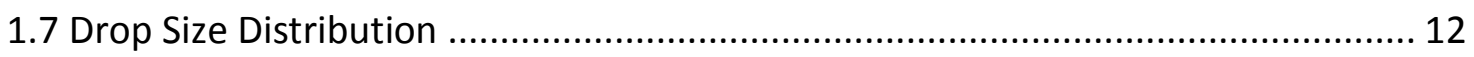

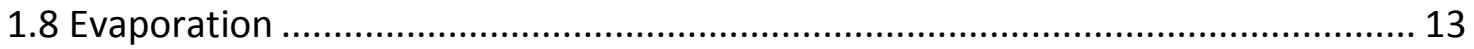

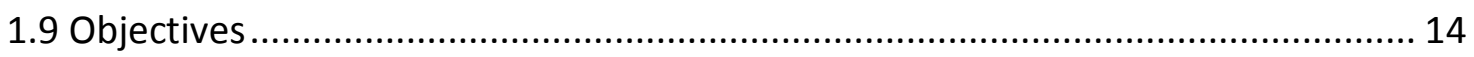

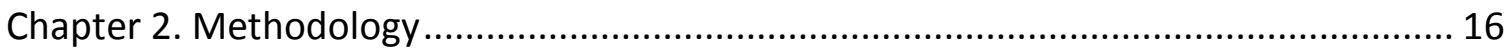

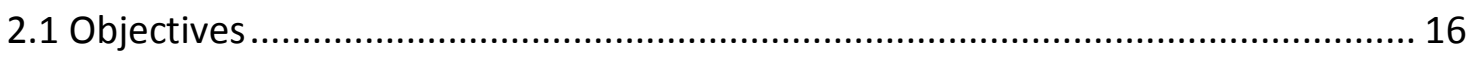

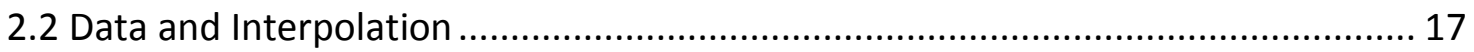

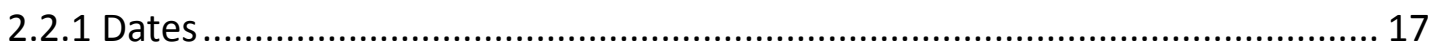

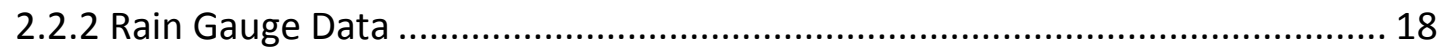


2.2.4 Timing

2.2.5 Conventional Formula Interpolation

2.3 High Resolution Model Data and the Trace Method

2.3.1 Drift and Evaporation

2.3.2 Model Data

2.3.3 Trace Method

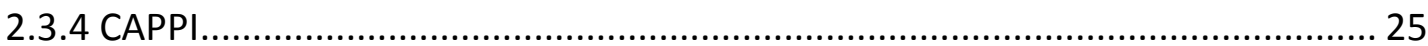

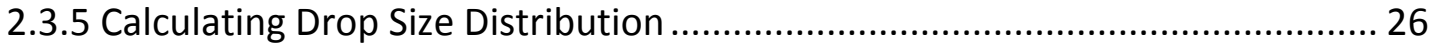

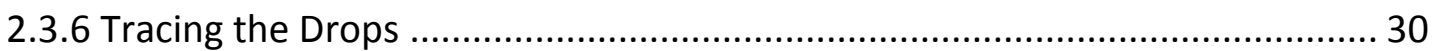

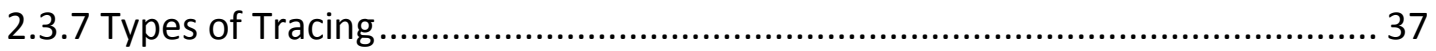

2.3.8 Estimating Rainfall with the Trace Method ................................................ 37

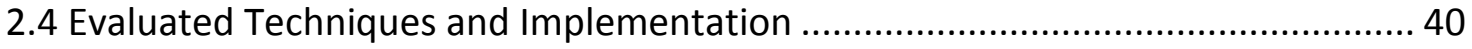

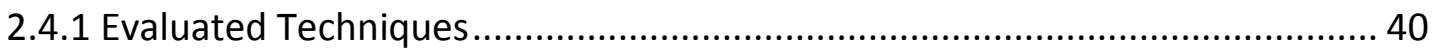

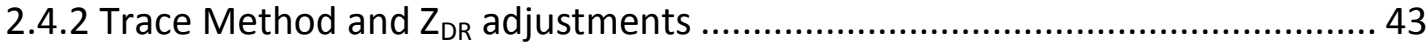

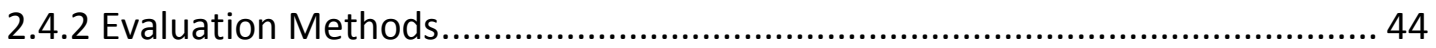

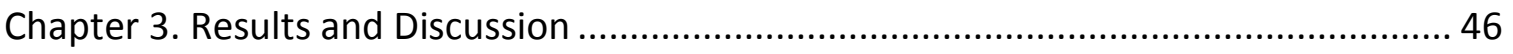

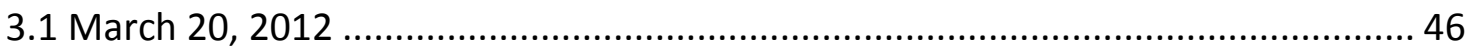

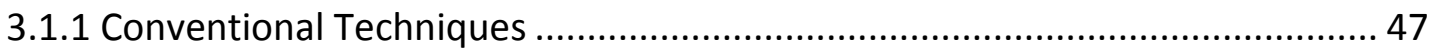




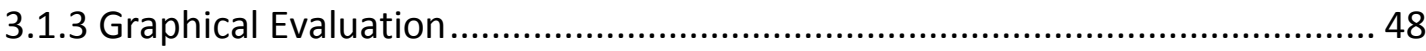

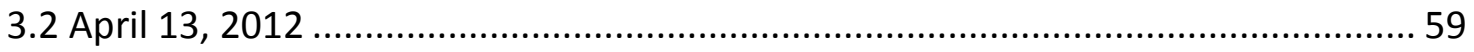

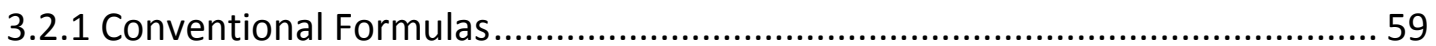

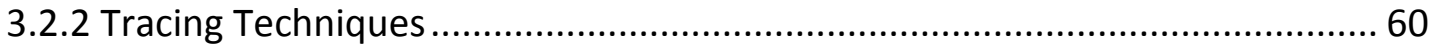

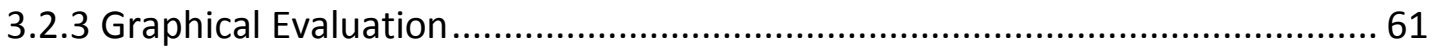

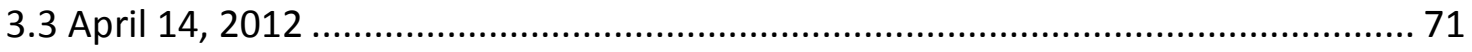

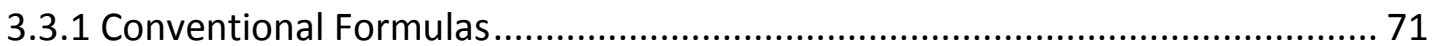

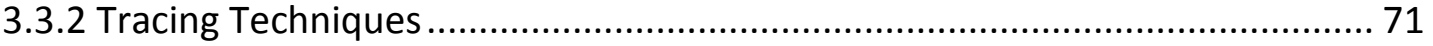

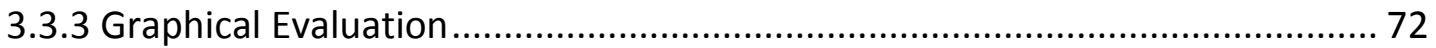

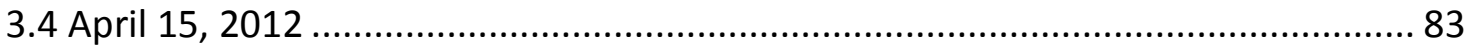

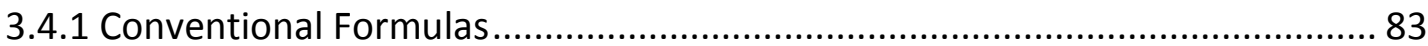

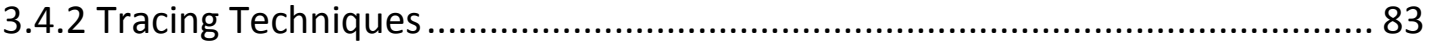

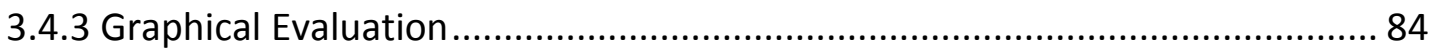

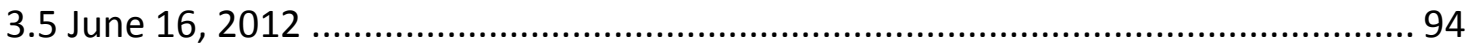

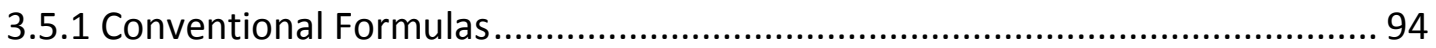

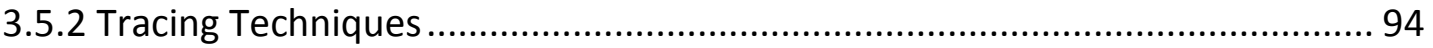

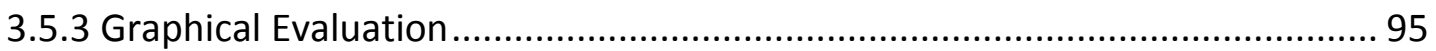

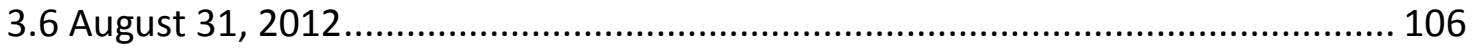


3.6.1 Conventional Formulas.

3.6.2 Tracing Techniques

3.6.3 Graphical Evaluation

3.7 September 7, 2012

3.7.1 Conventional Formulas

3.7.2 Tracing Techniques

3.5.3 Graphical Evaluation

3.8 October 13, 2012 130

3.8.1 Conventional Formulas 130

3.8.2 Tracing Techniques 130

3.5.3 Graphical Evaluation 131

3.9 March 10, 2013 141

3.9.1 Conventional Formulas. 141

3.9.2 Tracing Techniques 141

3.5.3 Graphical Evaluation. 142

3.10 April 10, 2013 153

3.10.1 Conventional Formulas. 153

3.10.2 Tracing Techniques 153

3.5.3 Graphical Evaluation.... 154 
3.11 April 17, 2013 165

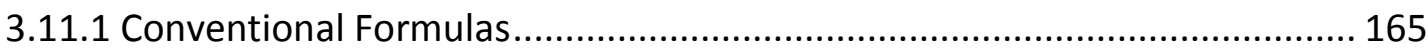

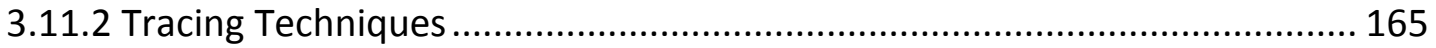

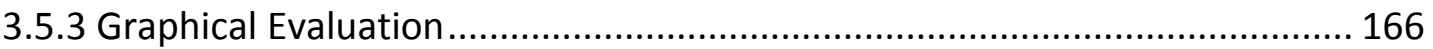

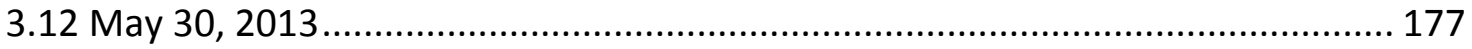

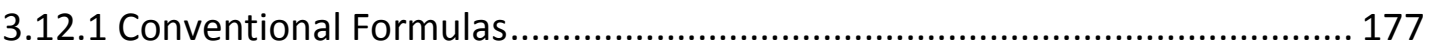

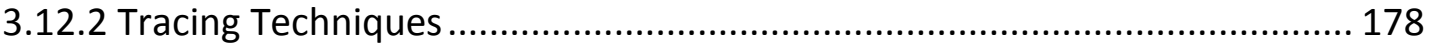

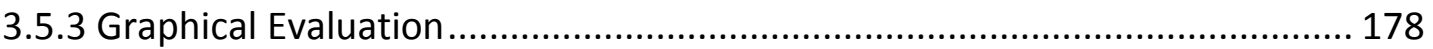

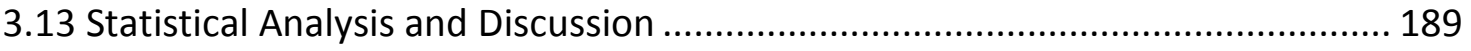

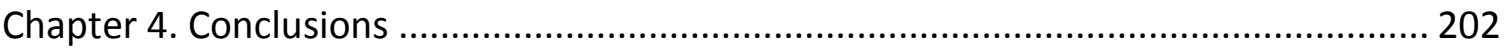

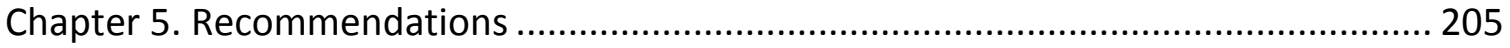

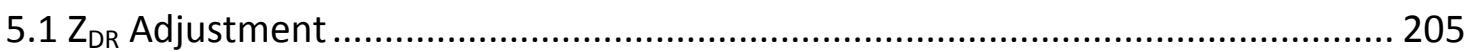

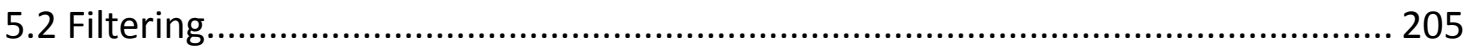

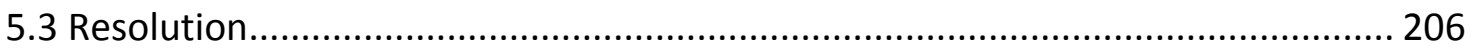

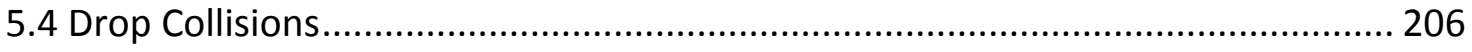

5.5 Evaporation Energy and Feedback into High Resolutions Models ...................... 206

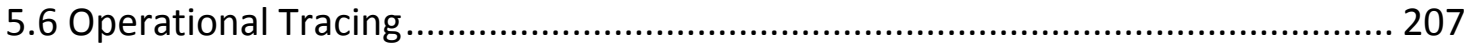

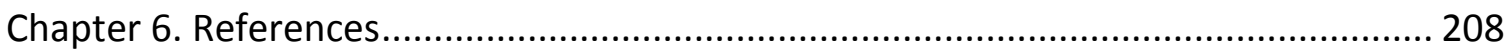

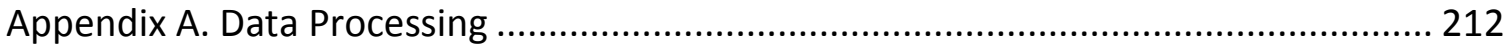


A.1 Radar Data.

Appendix B. Program Information 216

B.1 Program Description 216

B. 2 Hail Adjustment 216

Appendix C. Trace Information 218

C.1 Trace VBA Script 218

C.2 findinfo Script 224 


\section{LIST OF FIGURES}

Figure

Page

2.2. Site Locations Relative to KLSX (sky blue marker). 19

2.1. Site Locations in and around Columbia, Missouri. 19

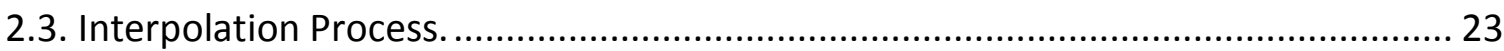

2.4. Theoretical Rainfall Rates with varying $Z$ and ZDR (mm/hour)............................ 29

2.5. Change in Theoretical Rainfall Rate (\%) with Varying $Z_{D R}$ and $\mu^{2}$ Coefficient........... 29

2.6. Change in Theoretical Rainfall Rate (\%) with Varying $Z_{D R}$ and $\mu$ Coefficient............ 30

2.7. Change in Theoretical Rainfall rate (\%) with Varying $Z_{D R}$ and $\mu^{0}$ Coefficient. ........... 30

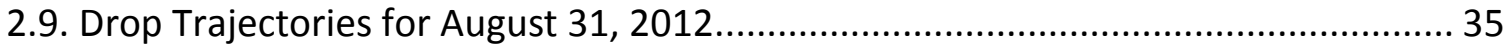

2.8. Drop Diameter as a Function of Time for August 31, 2012................................. 35

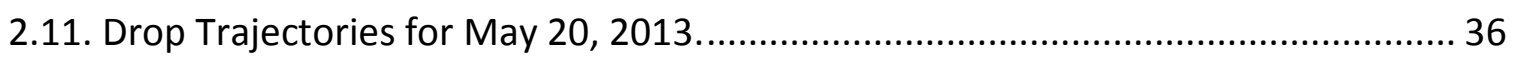

2.10. Drop Diameter as a Function of Time for May 20, 2013.................................. 36

2.12. Illustration of the Drop Size Interpolation Process........................................... 39

2.13. Sample X-Y Scatter plot of Radar Estimated Rainfall vs. Gauge Rainfall. ............... 45

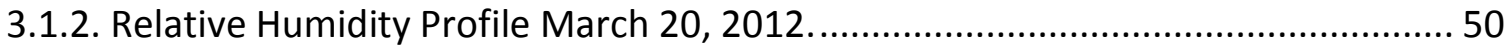

3.1.1. Surface Analysis 0300 UTC March 21, 2012................................................. 50

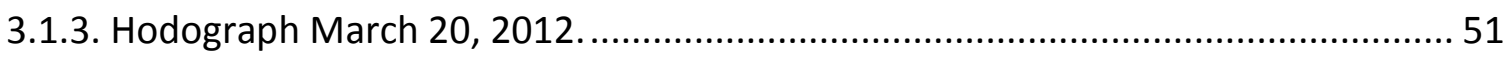

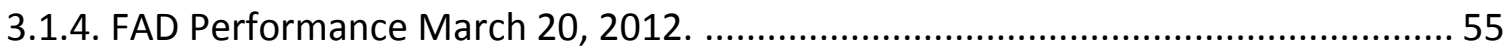

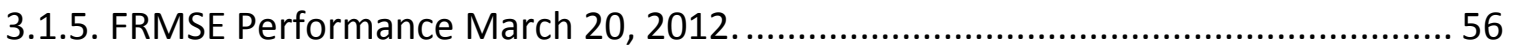

3.1.6. Radar Estimated Rainfall vs. Gauge Rainfall Scatterplots March 20, 2012. .......... 57 
3.1.7. Radar Estimated Rainfall vs. Gauge Rainfall Scatterplots March 20, 2012. 58

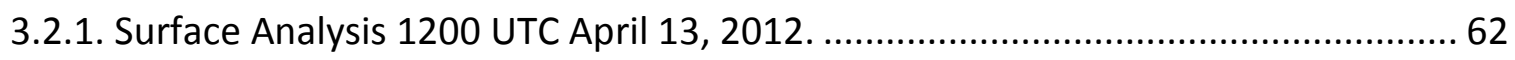

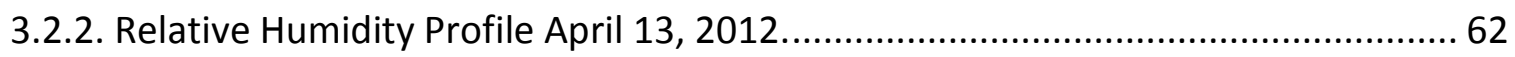

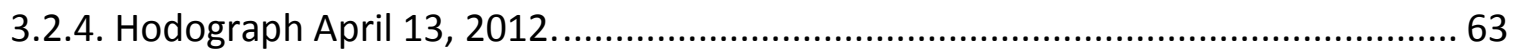

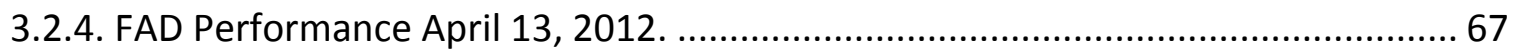

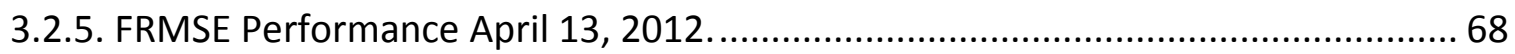

3.2.4. Radar Estimated Rainfall vs. Gauge Rainfall Scatterplots April 13, 2012. ............ 69

3.2.5. Radar Estimated Rainfall vs. Gauge Rainfall Scatterplots April 13, 2012. ............. 70

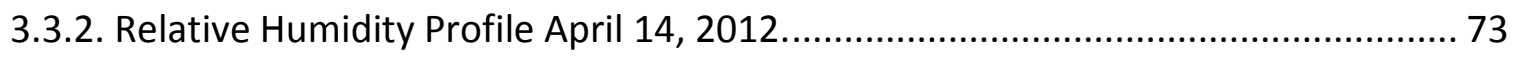

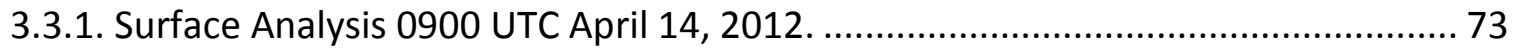

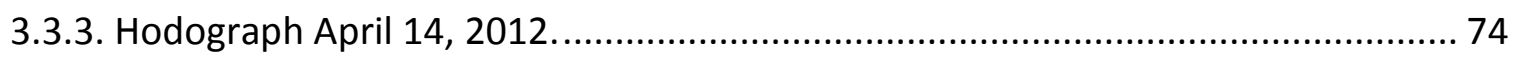

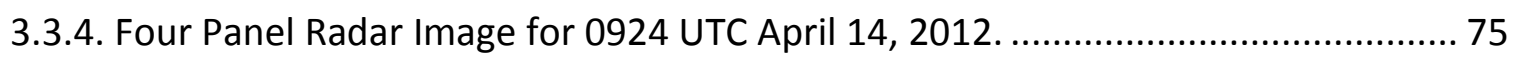

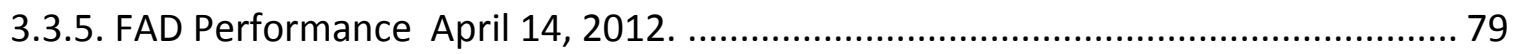

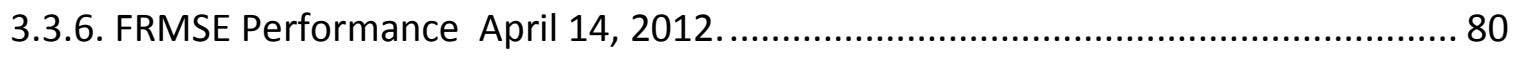

3.3.7. Radar Estimated Rainfall vs. Gauge Rainfall Scatterplots April 14, 2012. ............. 81

3.3.8. Radar Estimated Rainfall vs. Gauge Rainfall Scatterplots April 14, 2012. ............. 82

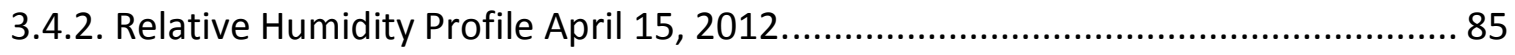

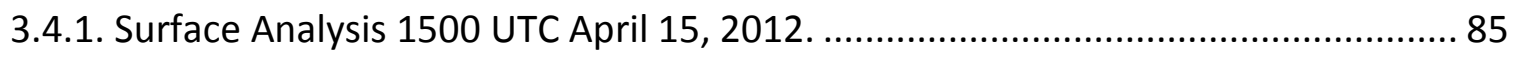

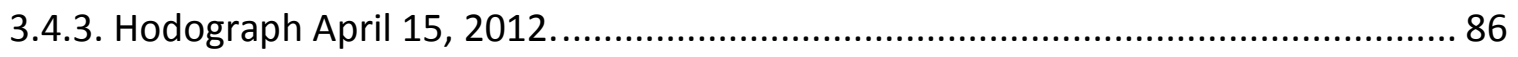

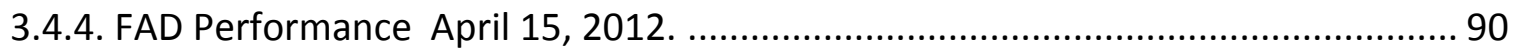

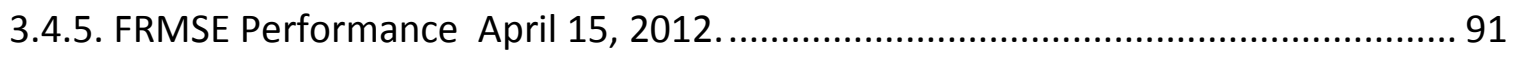

3.4.6. Radar Estimated Rainfall vs. Gauge Rainfall Scatterplots April 15, 2012. ............ 92 
3.4.7. Radar Estimated Rainfall vs. Gauge Rainfall Scatterplots April 15, 2012. 93

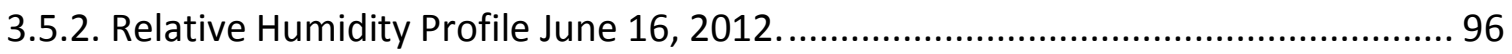

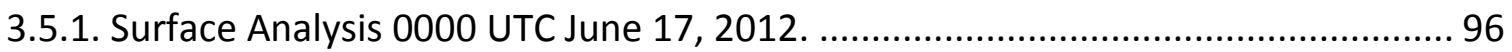

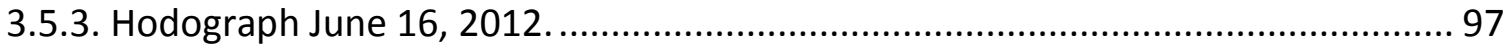

3.5.4. Four Panel Radar Image for 0021 UTC June 17, 2012.................................... 98

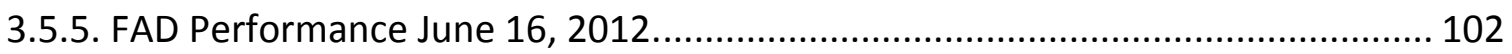

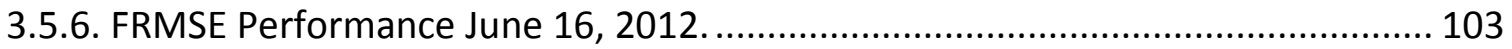

3.5.7. Radar Estimated Rainfall vs. Gauge Rainfall Scatterplots June 16, 2012. ........... 104

3.5.8. Radar Estimated Rainfall vs. Gauge Rainfall Scatterplots June 16, 2012. ........... 105

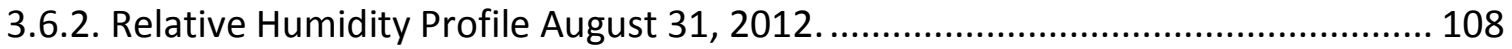

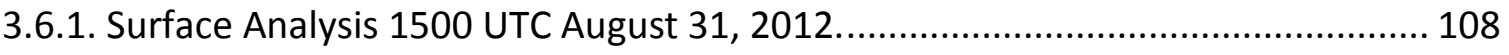

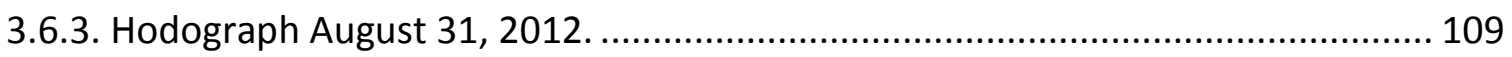

3.6.4. Four Panel Radar Image for 1537 UTC August 31, 2012. ............................... 110

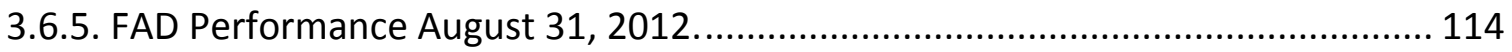

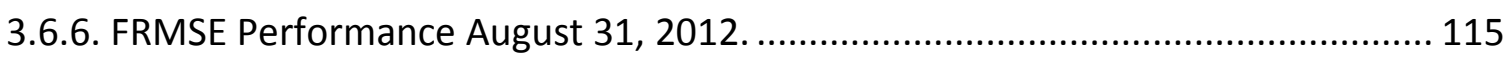

3.6.7. Radar Estimated Rainfall vs. Gauge Rainfall Scatterplots August 31, 2012........ 116

3.6.8. Radar Estimated Rainfall vs. Gauge Rainfall Scatterplots August 31, 2012......... 117

3.7.2. Relative Humidity Profile September 7, 2012.............................................. 120

3.7.1. Surface Analysis 2100 UTC September 7, 2012............................................ 120

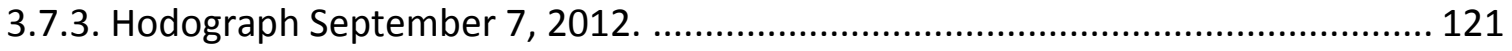

3.7.4. Four Panel Radar Image for 2107 UTC September 7, 2012............................. 122

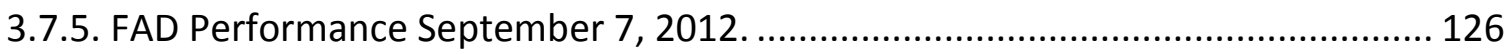


3.7.6. FRMSE Performance September 7, 2012 .

3.7.7. Radar Estimated Rainfall vs. Gauge Rainfall Scatterplots September 7, 2012.... 128

3.7.8. Radar Estimated Rainfall vs. Gauge Rainfall Scatterplots September 7, 2012.... 129

3.8.1. Surface Analysis 0300 UTC October 14, 2012 …........................................... 132

3.8.2. Relative Humidity Profile October 13, 2012 .................................................. 132

3.8.3. Hodograph October 13, 2012 ..................................................................... 133

3.8.4. FAD Performance October 13, 2012............................................................... 137

3.8.5. FRMSE Performance October 13, 2012....................................................... 138

3.8.6. Radar Estimated Rainfall vs. Gauge Rainfall Scatterplots October 13, 2012....... 139

3.8.7. Radar Estimated Rainfall vs. Gauge Rainfall Scatterplots October 13, 2012....... 140

3.9.2. Relative Humidity Profile March 10, 2013....................................................... 143

3.9.1. Surface Analysis 0600 UTC March 10, 2013................................................. 143

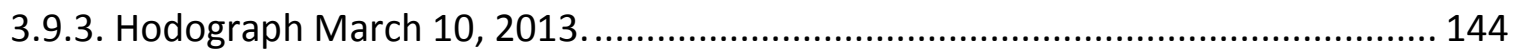

3.9.4. Four Panel Radar Image for 0631 UTC March 10, 2013................................... 145

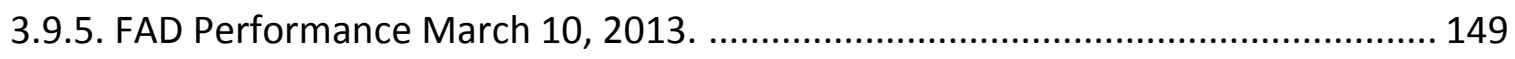

3.9.6. FRMSE Performance March 10, 2013.......................................................... 150

3.9.7. Radar Estimated Rainfall vs. Gauge Rainfall Scatterplots March 10, 2013......... 151

3.9.8. Radar Estimated Rainfall vs. Gauge Rainfall Scatterplots March 10, 2013. ......... 152

3.10.2. Relative Humidity Profile April 10, 2013...................................................... 155

3.10.1. Surface Analysis 2100 UTC April 10, 2013..................................................... 155

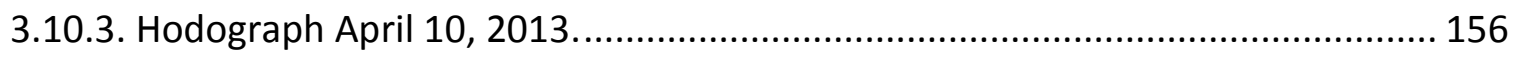

3.10.4. Four Panel Radar Image for 2154 UTC April 10, 2013.................................... 157 
3.10.5. FAD Performance April 10, 2013.

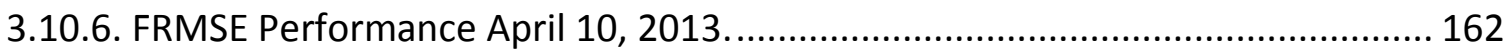

3.10.7. Radar Estimated Rainfall vs. Gauge Rainfall Scatterplots April 10, 2013.......... 163

3.10.8. Radar Estimated Rainfall vs. Gauge Rainfall Scatterplots April 10, 2013.......... 164

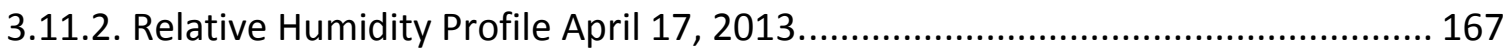

3.11.1. Surface Analysis 0000 UTC April 18, 2013................................................ 167

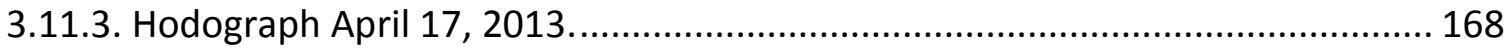

3.11.4. Four Panel Radar Image for 0743 UTC April 18, 2013.................................. 169

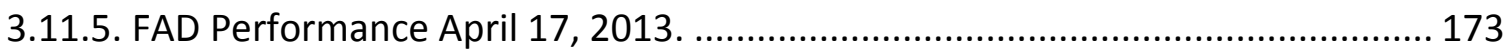

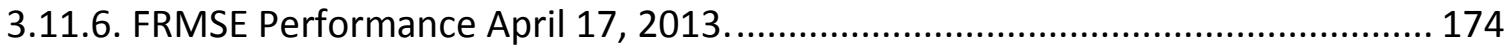

3.11.7. Radar Estimated Rainfall vs. Gauge Rainfall Scatterplots April 17, 2013.......... 175

3.12.2. Relative Humidity Profile May 30, 2013.................................................... 179

3.12.1. Surface Analysis 2100 UTC May 30, 2013................................................. 179

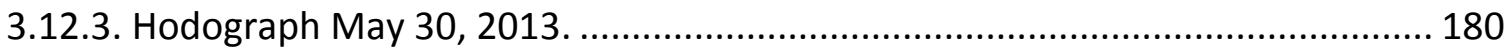

3.12.4. Four Panel Radar Image for 2044 UTC May 30, 2013.................................... 181

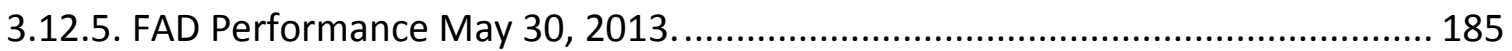

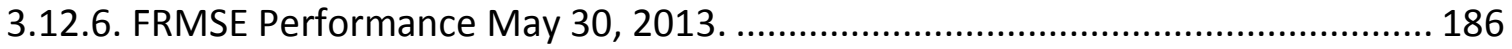

3.12.7. Radar Estimated Rainfall vs. Gauge Rainfall Scatterplots May 30, 2013........... 187

3.12.8. Radar Estimated Rainfall vs. Gauge Rainfall Scatterplots May 30, 2013........... 188

3.13.2. Median $F A D$ for the five trace techniques as a function of $Z_{D R}$ adjustment. ..... 199

3.13.1. Mean $F A D$ for the five trace techniques as a function of $Z_{D R}$ adjustment........ 199

3.13.3. Mean FRMSE for the five trace techniques as a function of $Z_{D R}$ adjustment.... 200 
3.13.4. Median FRMSE for the five trace techniques as a function of $Z_{D R}$ adjustment. . 200

3.13.6. Median bias for the five trace techniques as a function of $Z_{D R}$ adjustment. .... 201

3.13.5. Mean bias for the five trace techniques as a function of $Z_{D R}$ adjustment. ........ 201

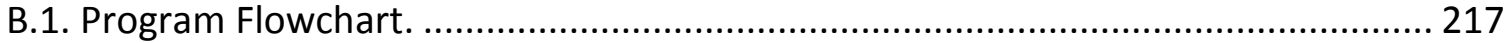




\section{LIST OF TABLES}

Table

Page

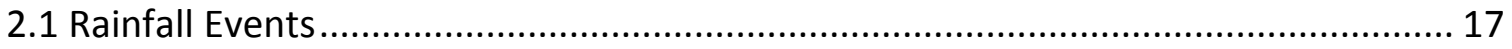

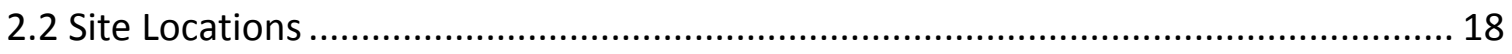

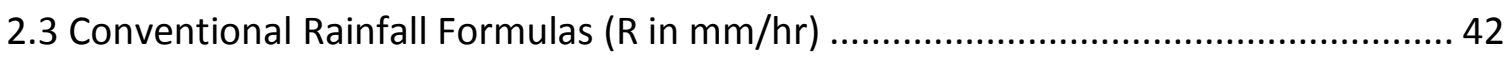

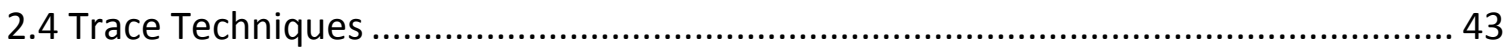

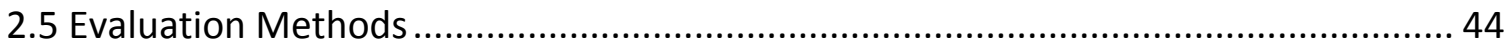

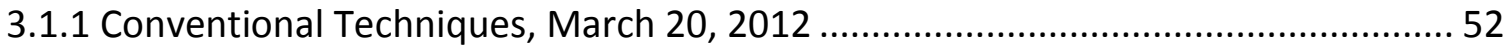

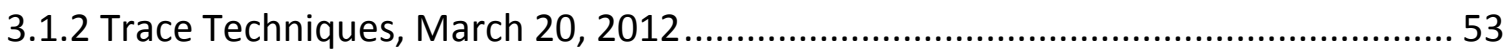

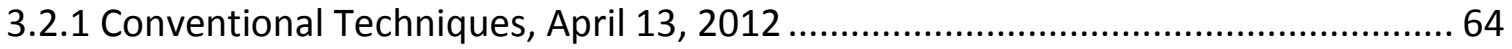

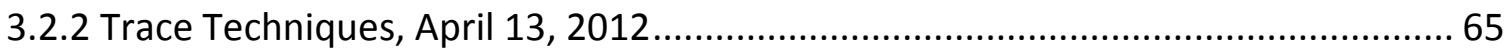

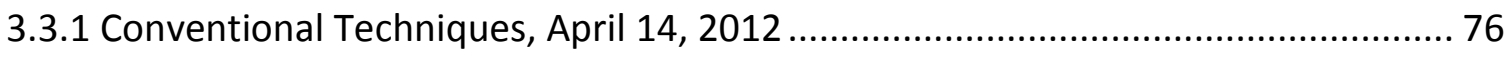

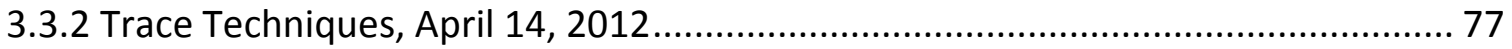

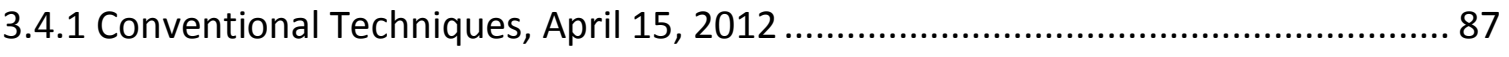

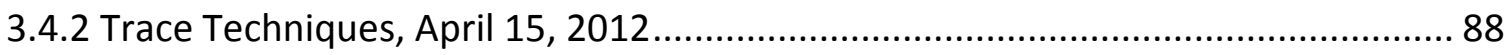

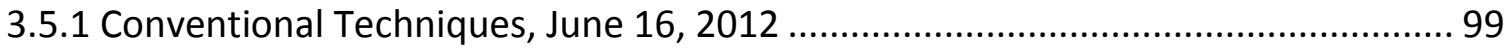

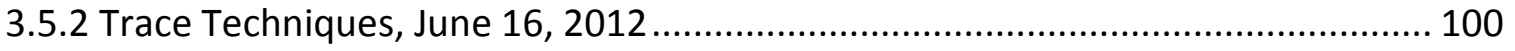

3.6.1 Conventional Techniques, August 31, 2012 ............................................... 111

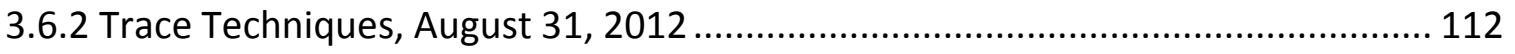

3.7.1 Conventional Techniques, September 7, 2012 ............................................ 123

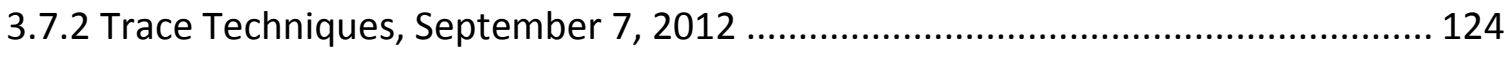


3.8.1 Conventional Techniques, October 13, 2012

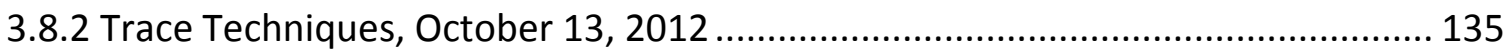

3.9.1 Conventional Techniques, March 10, 2013 ................................................... 146

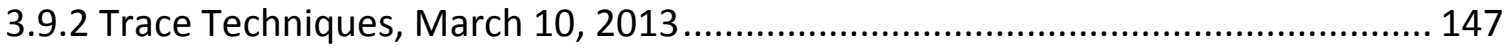

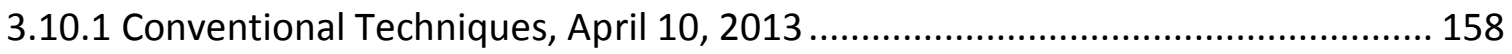

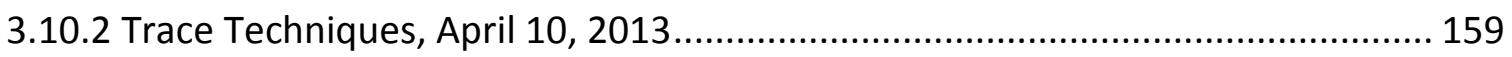

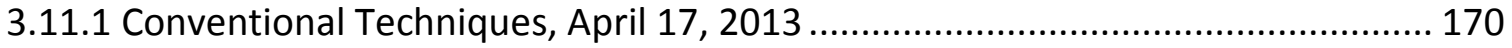

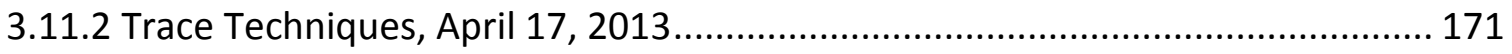

3.12.1 Conventional Techniques, May 30, 2013 ................................................. 182

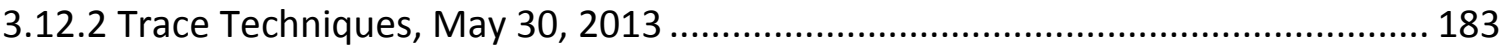

3.13.1 Conventional Formulas, FAD (\%) by Percentile ............................................. 193

3.13.2 Trace Techniques, FAD (\%) by Percentile ..................................................... 194

3.14.1 Conventional Formulas, FRMSE (\%) by Percentile.......................................... 196

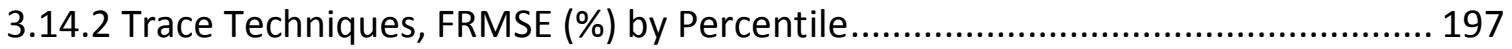




\section{Chapter 1. Introduction}

\subsection{Importance of Radar Estimation of Rainfall}

Knowledge of rainfall amounts is very useful information for several disciplines. With respect to environment, heavy rainfall can lead to chemical leaching of soils, causing toxic algal blooms and hypoxic conditions in nearby rivers. Agriculturally, Irrigation requirements and expected crop yields are also dependent on rainfall knowledge.

Immediate physical hazards are another area where rainfall information is important. Flash flooding and landslides are very dangerous to those caught unaware. River level forecasting is dependent on rainfall across very large areas. The level, location, and time of the crest can be estimated if enough data is available.

Evaluation of NWP (numerical weather prediction) models is another important function of rainfall information. These NWP model produce QPFs (quantitative precipitation forecasts). To evaluate the effectiveness of these models, a "truth" field is necessary. Surface rainfall estimations serve as this truth field. Model biases for location, intensity, and total rainfall can be quantified in this manner.

Two sources of rainfall data are generally available: rain gauge data and radar estimated rainfall. The in situ source, rain gauge data, is very limited in spatial coverage, especially in rural areas where agriculture is important. Radar estimated rainfall is remote sensing 
based, and can fill in gaps where no rain gauges are present. Areas surrounding

Columbia, Missouri, fit this description. Columbia is located in Central Missouri, an area far from the nearest radar location. This introduces several problems with radar rainfall estimation; many of these issues are examined in the following section.

\subsection{Sources of Uncertainty}

In order to improve rainfall estimation with radar, it is necessary to identify the difficulties associated with it. These challenges were aggregated by Villarini and Krajewski (Villarini and Krajewski 2010), referred to as VK10.

The first four error sources reviewed by VK10 were radar miscalibration, attenuation, ground clutter and anomalous propagation, and beam blockage. The first three of these sources were ignored in this study. They were ignored based on the assumption that either corrections had already been made by the weather service, or that the error caused by the problem was insignificant. Due to the relatively flat topography of the studied location, beam blockage was not expected to be an issue.

The fifth source of uncertainty was variability in the Z-R relation. Reflectivity $(Z)$ and rain rates (R) are dependent on drop size distribution (DSD), which the Z-R relationship depends on. In VK10 it was noted that numerous studies have been performed that match rain types with corresponding Z-R relations. Also noted were papers describing DSD as a function of geographic location and rainfall intensity (Tokay et al. 2001; Willis and Tattelman 1989). More than a dozen studies showing storm to storm and same storm changes in DSD were listed in VK10. Since VK10 dealt with single polarization 
radar, the effects of polarimetric parameters were not discussed. A more in-depth discussion of various Z-R relationships is found in section 1.3.

The sixth source of uncertainty was range degradation. Range degradation was described as a significant cause of underestimation of rainfall amounts at locations far from the radar in several studies. Range degradation was split into two categories: overshooting and beam broadening. Overshooting occurs when the lowest radar elevation angle is too high to capture information about the precipitation. The further one gets from the radar, the higher this lowest elevation angle is. Beam broadening is the reason for the lower resolution data generated for locations far from the radar. This low resolution at far ranges has two immediate consequences; small but intense features are averaged over large areas in all three dimensions, and different precipitation types with different reflectivity signatures can be lumped together. VK10 noted that studies have been performed quantifying bias in measured reflectivity due to beam broadening (Sánchez-Diezma et al. 2000). Little can be done about these two sources for uncertainty with current technology. Due to its location far from the nearest radar, Central Missouri is greatly affected by these issues. Deconvolution of overlapping beams provides limited improvement in these situations.

The seventh source was vertical variability of the precipitation system. The focus of this section was the transition of liquid precipitation types to frozen types higher up in the atmosphere. This transition results in changing reflectivity values due to the differences in shape, size, and physical properties of frozen precipitation. The layer just below the 
zero degree isotherm is known as the "bright band", due to the enhanced reflectivity within the layer. For this project, the events chosen took place in warm enough atmospheres that this problem was not an issue.

Also discussed in the vertical variability section was hail contamination. Due to the dependency of reflectivity on the sixth power of drop diameter, medium and large hailstones can cause extreme increases in reflectivity. When applied to simple Z-R relationships, this causes unrealistically high instantaneous rain rates. VK10 noted that improvements were found when special Z-R formulas optimized for hail were combined with hail detection schemes (Auer 1994). A form of hail detection/adjustment was utilized for this project.

The eighth source for uncertainty was vertical air motion and precipitation drift. Vertical air motion was a factor that went unaccounted for in this project. Vertical air motion has the biggest effect on smaller drops, as they have the smallest terminal velocity. The areas where vertical air motion has the greatest effect would theoretically be updrafts within thunderstorms. This would be a highly localized phenomenon.

Precipitation drift was a factor considered for this project. In VK10 it was noted that proposals have been made to account for horizontal displacement of drops due to wind drift (Dalezios and Kouwen 1990). Dalezios and Kouwen did not find any significant impact of wind drift on hourly time scales. Further discussion of precipitation drift is conducted in section 1.6 . 
Temporal sampling error was the final source for uncertainty described by VK10.

Unfortunately, little can be done about this without technological upgrades to current radar systems. Finding the optimal interpolation method is the best that can be hoped for.

\subsection{Z-R Relationships}

In their 1948 paper, Marshall and Palmer postulated that the DSDs are exponential in nature (Marshall and Palmer 1948). The exponential DSD was used to derive a relationship between $\mathrm{Z}$ and $\mathrm{R}$. The revised Marshall-Palmer Z-R relationship is given by

$$
Z=200 R^{1.6}
$$

For Equation 1.1, $\mathrm{Z}$ has units of $\mathrm{mm}^{6} / \mathrm{m}^{3}$ and $\mathrm{R}$ has units of $\mathrm{mm} /$ hour.

More recently, the WSR-88D rainfall algorithm was developed (Fulton et al. 1998). The WSR-88D algorithm has several quality control features to account for many of the sources of uncertainty listed earlier. These include corrections for partial beam blockage, ground clutter, and anomalous propagation.

The default formula used for the WSR-88D algorithm is shown in Equation 1.2.

$$
Z=300 R^{1.4}
$$

In some tropical locations the formula used is Equation 1.3 (Rosenfeld et al. 1993).

$$
Z=250 R^{1.2}
$$

For the WSR-88D rainfall algorithms the rain rates are computed on a $1^{\circ} \times 1 \mathrm{~km}$ grid, and averaged in pairs along the radials. The WSR-88D rainfall algorithm also utilizes a 
hail cap. The maximum reflectivity is capped at a value of 51 to $55 \mathrm{dBZ}$, changing based on the location. This cap excludes extreme reflectivity values that can only be caused by hail.

\subsection{Dual Polarization}

Dual polarization utilizes horizontally and vertically polarized radiation to obtain information about the hydrometeors within an atmospheric column that would otherwise be unavailable. The non-spherical shape of raindrops larger than roughly one millimeter causes the drops to interact differently with the horizontally and vertically polarized radiation. This difference in interaction can be used to calculate the differential reflectivity, specific phase differential, and cross-correlation coefficient. These parameters are dependent on the DSD, and knowledge of them allows for estimates of the DSD to be obtained.

The effectiveness of the polarimetric rainfall algorithms has been evaluated in many studies (Ryzhkov et al. 2005), referred to as RGS05, (Ryzhkov and Zrnić 1996). These formulas have been found to be more effective than the simple Z-R formulas. Most of the rainfall relationships tested by RGS05 were also evaluated for this project. In addition, the three evaluation parameters from RGS05, fractional bias, fractional root mean square error, and fractional standard deviation, were calculated for this project.

RGS05 aggregated several drop shape formulas, and utilized them to generate the tested rainfall relations. Drop shape formulas are given as the raindrop axis ratio as a 
function of drop diameter. Larger drops tend to have lower axis ratios, meaning they are elongated in the horizontal direction.

The first drop shape formula listed in RGS05 was based on the equilibrium drop shapes from Beard and Chuang (Beard and Chuang 1987). This formula was

$$
\begin{gathered}
a / b=1.0048+0.00057 * D-0.02628 * D^{2}+0.003682 * D^{3}- \\
0.0001677 * D^{4} .
\end{gathered}
$$

Here $a / b$ is the axis ratio and $D$ is the equivolume diameter. The second drop shape formula listed accounted for drop oscillations, which in unsteady flow have the potential to disrupt the equilibrium drop shape (Andsager et al. 1999). The formula was given by

$$
a / b=1.012-0.01445 * D-0.01028 * D^{2} .
$$

The third drop shape formula came from (Brandes et al. 2002). This formula was suited for a more tropical environment and was given by

$$
\begin{gathered}
a / b=0.9951+0.02510 * D-0.03644 * D^{2}+0.005303 * D^{3}- \\
0.0002492 * D^{4} .
\end{gathered}
$$

The final drop shape formula listed in RGS05 was the Goddard's axis ratio (Goddard et al. 1995).

$$
a / b=1.075-0.065 * D-0.036 * D^{2}+0.0004 * D^{3} .
$$

Over the range of expected raindrop sizes (zero to six millimeters in diameter), the difference in axis ratios between the first three formulas was not significant. The axis ratio variation at its maximum barely exceeded $3 \%$. 
Two types of evaluations were done in RGS05, point evaluations and areal evaluations. The point evaluations were made by comparing radar rainfall estimation with gauge results for a $1^{\circ} \mathrm{X} 1 \mathrm{~km}$ region centered on the rain gauge. Areas evaluations were done by comparing average total radar rainfall estimation with average total gage accumulation across all of the rain gauges. The time scale where evaluations were made was one hour. The gauge network consisted of 42 rain gauges located between 85 and $50 \mathrm{~km}$ to the southwest of the radar.

The polarimetric formulas tested in RGS05 were split into two groups. The first group consisted of formulas derived from statistics of DSD measurements in central Oklahoma, and varying drop shape formulas. The second group was made up of the most recent polarimetric formulas from the literature at the time (Brandes et al. 2002; Bringi and Chandrasekar 2001; Illingworth and Blackman 2002).

Another type of formula was introduced in RGS05: the synthetic formula. The synthetic formula was designed to handle rainfall situations where the intensity of the rain varies significantly. Three separate equations were utilized; the reflectivity value determined which equation was used. It was found in RGS05 that the synthetic formula performed the best out of all the polarimetric formulas, and significantly better than the simple Z-R relations. A version of the synthetic formula was tested for this project, and more details on the implementation are presented in the methodology section. 


\subsection{Raindrop Terminal Velocity}

One fundamental problem with generating rainfall estimations from radar data is that it provides information about the atmospheric concentration of hydrometeors at a level high above the surface. Many things can happen to the drops while they fall through the atmosphere. They can translate horizontally, collide with other drops, and split into smaller drops. Evaporation reduces the size of the drops as they fall through the atmosphere.

High resolution model data provides the possibility of accounting for some of these factors. To quantify some of the factors affecting the trajectory and size of raindrops, knowledge of several atmospheric variables is required. These include horizontal wind, environmental temperature, and environmental humidity.

The terminal velocity of raindrops is a key component for estimating raindrop trajectories. In 1949, Gunn and Kinzer published a paper showing experimental terminal velocities of drops ranging in size from 0.1 millimeters in diameter up to 5.8 millimeters in diameter (Gunn and Kinzer 1949). Also shown were calculated Reynolds Numbers and drag coefficients. Gunn and Kinzer's measurements were made at the surface; subsequent papers dealt with the velocities of drops aloft, where the air is less dense (Foote and Du Toit 1969). Foote and Du Toit describe the correction factor shown in Equation 1.8.

$$
V(D)=V_{0}(D)\left(\frac{\rho_{0}}{\rho}\right)^{0.4}
$$


In Equation 1.8, $V_{0}(D)$ is the terminal velocity at air density $\rho_{0}$ and $\rho$ is the air density at the point where $V(D)$ is being calculated. This equation is actually a simpler, slightly less accurate, version of the more complex equation shown in Foote and Du Toit's 1969 study.

Numerous equations fitted to match the empirical fall velocities have been described (Best 1950; Boxel 1997; Gossard et al. 1990; Gossard et al. 1992; Uplinger 1981). These equations generally have an exponential term. The Gossard equation for drops falling faster than 2.75 meters per second (1990) is shown in Equation 1.9.

$$
V_{0}=9.65-10.3 * \exp (-0.6 D)
$$

Equation 1.9 is relevant for drops falling near the surface. $D$ is the drop diameter in millimeters and $V_{t}$ is the terminal velocity in meters per second. Boxel makes a slight modification to prevent the smallest drops from having negative terminal velocities in Equation 1.10.

$$
V_{0}=9.65 *(1-\exp (-0.53 * D))
$$

Equation 1.10 ended up being chosen for this project due to its simplicity and the problematic implication of allowing negative terminal velocities. Terminal velocity values from Equation 1.10 differ from their empirical counterparts from Gunn and Kinzer by roughly $5 \%$ for three millimeter diameter drops, $3 \%$ for two millimeter diameter drops, and $1.5 \%$ for one millimeter diameter drops. 


\subsection{Precipitation Drift}

Accounting for horizontal hydrometeor movement has been the subject of several studies (Lack 2004; Lauri et al. 2011; Mittermaier et al. 2004). Lack 2004 utilized radar derived velocity data procured from the Sydney 2000 World Weather Research Programme's (WWRP) Forecast Demonstration Project (FDP) (Keenan et al. 2003). Results from Lack indicated that precipitation drift had the greatest potential for effect when using high resolution data and for situations where low-level directional wind shear was present. Using humidity information to account for drop evaporation was one of the items listed for future work within Lack. The other two studies made no mention of evaporation.

Lauri et al. 2011 and Mittermaier et al. 2004 were more focused on the drift of frozen precipitation above the melting layer. Snowflakes generally fall slower than raindrops, and are thus more affected by wind drift. It was noted in Mittermaier that numerically modeled wind speeds were of sufficient accuracy to yield improvements with the drift scheme they devised. A simple hit, miss, and false alarm evaluation method was used to determine effectiveness in Mittermaier.

Lauri's focus was also on the potential for wind drift to affect frozen hydrometeors. Included in Lauri were radar-to-gauge comparisons illustrating a significant improvement when hydrometeor advection was accounted for. Both Lauri and Mittermaier worked at higher latitudes, where the radar beam was more likely to hit frozen precipitation. 


\subsection{Drop Size Distribution}

Any radar-derived rainfall estimation scheme must take into account the varying size and concentration of raindrops. The purely exponential DSD devised by Marshall and Palmer has largely been replaced by the gamma DSD (Ulbrich 1983). The gamma DSD is given by

$$
n(D)=n_{0} * D^{\mu} * \exp (-\Lambda D)
$$

For the gamma distribution, $n_{0}$ is the intercept parameter, $\mu$ is the shape parameter, and $\Lambda$ is the slope parameter. $n(D)$ is the number of drops of diameter $D$. The shape parameter determines the magnitude of deviation from the exponential distribution. A $\mu$ value of zero makes the gamma distribution equal to the exponential distribution (Tokay et al. 2001).

A method for using the three radar parameters, $Z, Z_{D R}$, and $K_{D P}$, to obtain the necessary constants within the gamma distribution has been developed (Gorgucci et al. 2002). $K_{D P}$ values, however, are extremely noisy. To eliminate dependence on $\mathrm{K}_{\mathrm{DP}}$, Brandes et al. introduced a "constrained-gamma method" (Brandes et al. 2004). This constrainedgamma method made use of empirical dependencies of $\Lambda$ on $\mu$. With only two unknowns to be found, the constrained-gamma method only requires two radar parameters to estimate the expected DSD. 


\subsection{Evaporation}

Literature dealing with raindrop evaporation calculations is relatively limited in nature.

In 1951 Gunn and Kinser published a paper detailing an analytic solution to evaporation for a falling drop (Kinzer and Gunn 1951). The rate of mass change per unit time was given by

$$
\frac{d m}{d t}=4 \pi r D_{v} \Delta \rho_{v} *\left(1+\frac{F r}{s^{\prime}}\right)
$$

In Equation 1.12, $\mathrm{m}$ was the mass of the raindrop, $r$ was the drop radius, $D_{v}$ was the diffusivity of water vapor in air, $\Delta \rho_{v}$ was the vapor density difference, and $1+\frac{F a}{s^{\prime}}$ was the ventilation coefficient term.

Recent updates to this formula have been made (Li and Srivastava 2001). The rate of evaporation in the Li and Srivastava study was calculated as

$$
\frac{d m}{d t}=4 \pi r D_{v} \Delta \rho_{v} f_{v}
$$

The ventilation coefficient, $f_{v}$, was calculated as

$$
f_{v}=0.78+0.308\left(\frac{v}{D_{v}}\right)^{\frac{1}{3}}\left(\frac{V D}{v}\right)^{\frac{1}{2}}
$$

In these equations, $\mathrm{m}$ is the mass of the rain drop, $\mathrm{V}$ is the terminal velocity of the drop, $v$ is the kinematic viscosity of air, $D_{v}$ is the diffusivity of water vapor in air, $r$ is the drop radius, $\mathrm{D}$ is the drop diameter, and $\Delta \rho_{v}$ is the difference in vapor density between the drop surface and the environment. 
The Li and Srivastava study established a parameter, $D^{*}$, that was defined as the diameter of raindrop that would just completely evaporate after traveling through a layer of the atmosphere with specified qualities. This parameter essentially corresponded to the "drying power" of the layer. The study goes on to detail the relationship between $D^{*}$ and several other parameters, including evaporation induced changes in DSDs and evaporation-related errors in rainfall estimation with Z-R and ZDRZ-R relations.

A more focused examination of the effect of evaporation on polarimetric radar parameters was conducted in 2010 (Kumjian and Ryzhkov 2010). The theoretical effects of evaporation on $\mathrm{Z}, \mathrm{Z}_{\mathrm{DR}}$, and $\mathrm{K}_{\mathrm{DP}}$ were discussed in this study. Evaporation was calculated to cause substantial alterations in each of the three parameters. $Z$ and $K_{D P}$ were found to be reduced by evaporation, while ZDR was increased. Since conventional formulas for rainfall estimation are directly proportion to powers of $Z$ and KDP, but inversely proportional to powers of $Z_{D R}$, each of these three changes from evaporation was expected to result in rainfall overestimation. The study noted that the effects of evaporation were generally reduced as the rainfall rate increased, and that $K_{D P}$ was the parameter most affected.

\subsection{Objectives}

There were two primary objectives for this project. The first was to determine the best method for radar rainfall estimation over Central Missouri. The second objective was to 
evaluate whether the introduction of high resolution model data has the potential to improve the radar rainfall estimation process.

A number of different conventional formulas were considered for the first objective. The conventional formulas were simple functions of the $Z, Z_{D R}$, and $K_{D P}$ parameters.

The "trace" method was introduced as a way for high resolution model data to potentially improve radar rainfall estimation. The trace method relied on tracing all raindrops hitting the surface at a point back to the locations in space and time at which the radar detected them. Evaporation and horizontal drift were both utilized in the tracing process.

Variations on the trace method were used along with the conventional formulas when the determination of best method was in question. These variations consisted of different combinations of evaporation and horizontal drift. Also included in the trace method was a version where raindrops were assumed to instantaneously reach the ground. Testing each of these variations allowed isolation of the effects a particular parameter had on the effectiveness of the method.

To the author's knowledge, all estimation of raindrop evaporation to date has been theoretical in nature. Evaluations of the trace techniques designed for this project provided a physical test of raindrop evaporation theory. This area of study is at the very least extremely limited in nature. 


\section{Chapter 2. Methodology}

\subsection{Objectives}

There were two primary objectives for the study. The first was to determine the best method for radar derived rainfall estimation in Central Missouri. The second objective was to evaluate whether high resolution model data could be utilized to improve radar derived rainfall estimation. In order to complete the objectives required for the study, two things were necessary: a place to store data and the ability to perform computations on the data. Visual Basic for Applications (VBA) and Microsoft Excel were chosen to perform these functions. VBA is a powerful tool for data manipulation when combined with spreadsheet software like Excel. The VBA program created for this project was designed with three requirements in mind. The first requirement was speed; the program would have to perform its calculations in a reasonable amount of time. The second requirement was flexibility. To fulfill the flexibility requirement, the program would have to be able to easily switch between rainfall estimation formulas, events, and locations for evaluation. The final requirement fell upon the results generated by the program. The results the program produced needed to be both graphical and numeric, and easy to interpret. 


\subsection{Data and Interpolation}

\subsubsection{Dates}

Evaluation of the effectiveness of radar-related rainfall estimation techniques first requires rain gauge and radar data. The dates used for the study were focused on major rainfall events over the years 2012 and 2013 in Central Missouri. Criteria for selection included availability of radar data and rain gauge data, as well as storm type. A wide variety of storm types was necessary to effectively evaluate the estimation methods.

The final dates chosen are listed in Table 2.1.

Table 2.1 Rainfall Events

\begin{tabular}{|c|c|c|c|c|c|}
\hline Event & Start Time & End Time & $\begin{array}{c}\text { Duration } \\
\text { (hours) }\end{array}$ & $\begin{array}{l}\text { Mean Site } \\
\text { Precipitation } \\
(\mathrm{mm})\end{array}$ & Notes \\
\hline March 20, 2012 & $3 / 2021$ UTC & $3 / 2121$ UTC & 24 & 36.91 & - \\
\hline April 13, 2012 & 4/13 05 UTC & 4/13 13 UTC & 8 & 6.24 & - \\
\hline April 14, 2012 & 4/14 02 UTC & 4/14 10 UTC & 8 & 17.58 & - \\
\hline April 15, 2012 & 4/15 11 UTC & 4/15 19 UTC & 8 & 4.85 & - \\
\hline June 16, 2012 & 6/16 19 UTC & 6/17 03 UTC & 8 & 20.73 & - \\
\hline August 31, 2012 & 8/31 12 UTC & 8/31 22 UTC & 10 & 34.66 & Isaac \\
\hline September 7, 2012 & 9/7 20 UTC & 9/8 01 UTC & 5 & 12.36 & Large Hail \\
\hline October 13, 2012 & 10/13 19 UTC & 10/14 05 UTC & 10 & 25.48 & - \\
\hline March 10, 2013 & 3/10 02 UTC & 3/10 10 UTC & 8 & 11.23 & - \\
\hline April 10, 2013 & 4/10 20 UTC & 4/11 4 UTC & 8 & 27.83 & - \\
\hline April 17, 2013 & 4/17 20 UTC & 4/18 11 UTC & 15 & 36.52 & Large Hail \\
\hline May 30, 2013 & 5/30 09 UTC & 5/31 17 UTC & 32 & 106.81 & - \\
\hline
\end{tabular}




\subsubsection{Rain Gauge Data}

Rain gauge data was obtained from nine locations around Central Missouri. Figure 2.1 and Figure 2.2 show the spatial distribution of these locations. Table 2.2 shows the coordinates of these sites as well as their distance from the radar (KLSX). These sites used tipping bucket rain gauges to measure rainfall at five minute intervals. Rainfall measurements were recorded in inches and converted to millimeters. Rain gauge data were assumed to be the "ground truth" for the purpose of this study. The rain gauge data were obtained from the Commercial Agriculture Automated Weather Station Network managed by University of Missouri Extension and Interdisciplinary Laboratory. All rain gauge data was stored within the Excel file where the main program was executed from.

Table 2.2 Site Locations

\begin{tabular}{lllll} 
Site & Latitude & Longitude & $\begin{array}{l}\text { Radar Distance } \\
(\mathrm{km})\end{array}$ & Elevation $(\mathrm{m})$ \\
\hline 1 & 39.023633 & -92.246017 & 140.1 & 225.6 \\
2 & 38.982733 & -92.279300 & 141.8 & 213.8 \\
3 & 38.948183 & -92.305350 & 143.3 & 195.8 \\
4 & 38.927750 & -92.342444 & 146.0 & 186.8 \\
5 & 38.914117 & -92.400183 & 150.7 & 171.9 \\
6 & 38.897236 & -92.218070 & 134.9 & 268.8 \\
7 & 38.929237 & -92.321297 & 144.3 & 186.2 \\
8 & 38.906992 & -92.269976 & 139.5 & 251.8 \\
9 & 38.942301 & -92.320395 & 144.4 & 235.0 \\
\hline
\end{tabular}




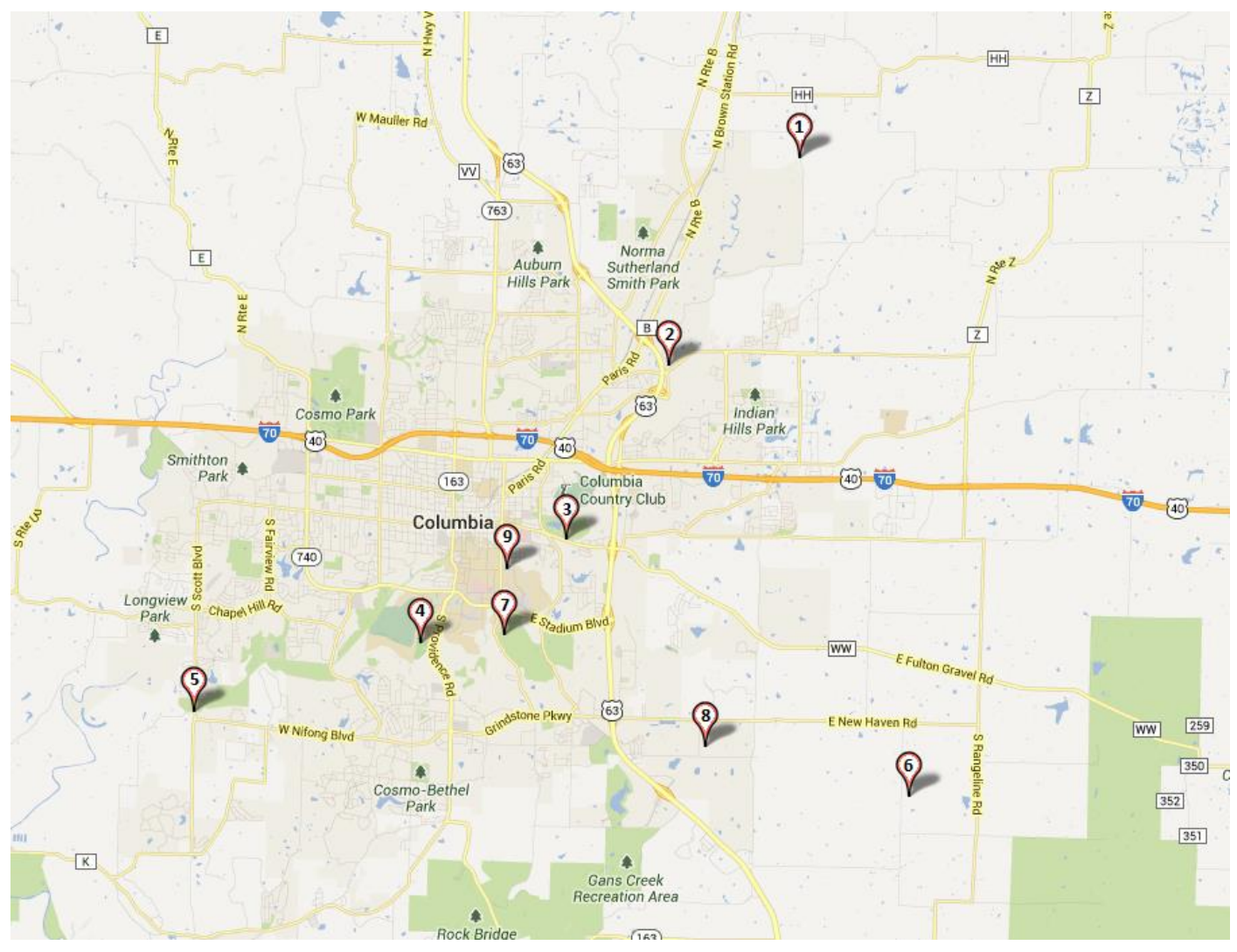

Figure 2.1. Site Locations in and around Columbia, Missouri.

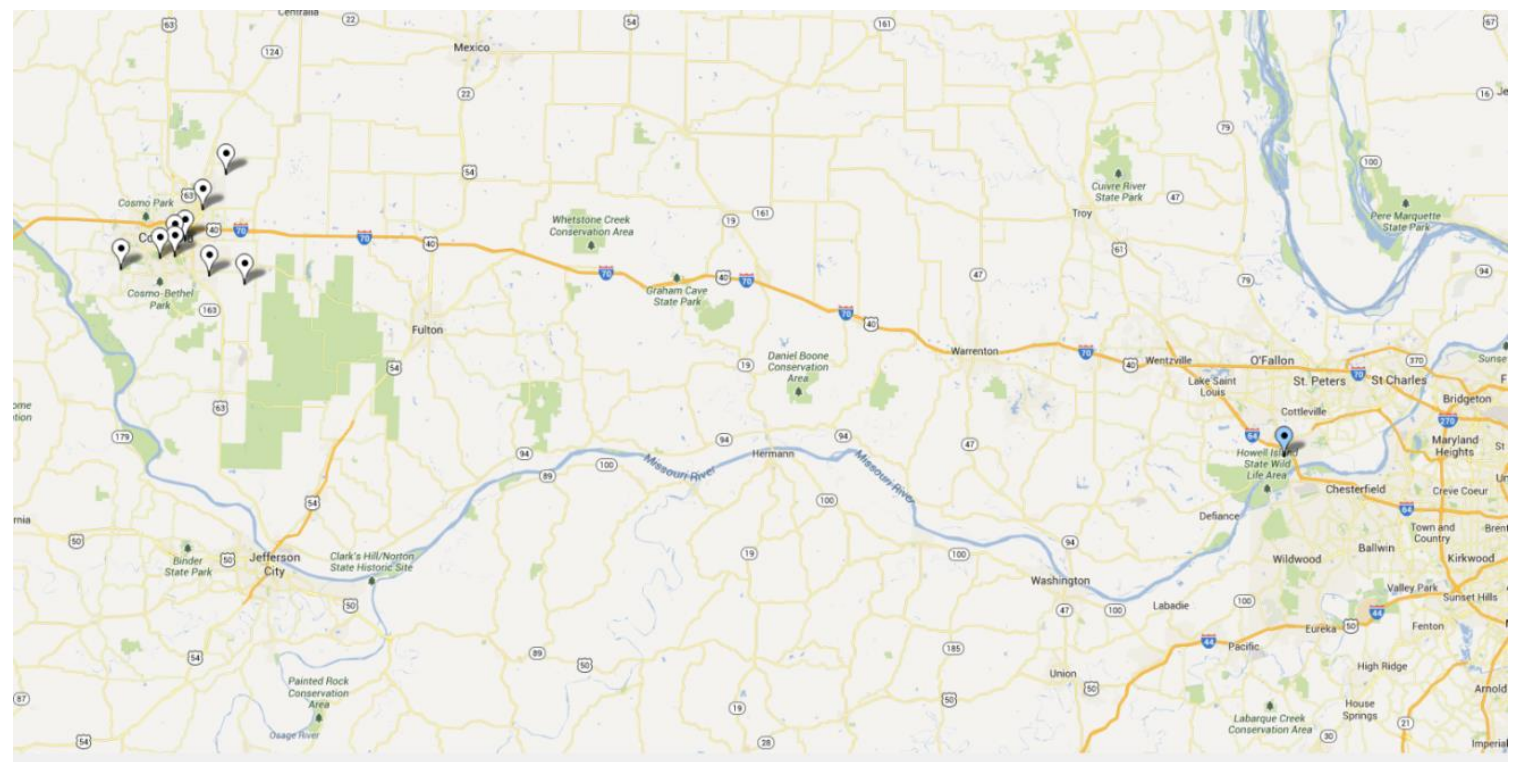

Figure 2.2. Site Locations Relative to KLSX (sky blue marker). 


\subsubsection{Radar Data}

Radar data was Level 2 WRS-88D for KLSX downloaded from NCDC. The data was processed using the Warning Decision Support System (Lakshmanan et al. 2007). The source radar data was converted to a Cartesian CAPPI grid using the w2merger process (Lakshmanan et al. 2006) to produce $1 \mathrm{~km} \mathrm{X} 1 \mathrm{~km}$ grids at nominal five minute intervals (not coincident with scan frequency). Radar files for $Z$ and $Z_{D R}$ were obtained, while $K_{D P}$ was obtained through calculation of the gradient of $\phi_{D P}$. This was done using a fixed kernel size resulting in noisy and inconsistent values; the kernel should be adjusted depending on the radar pulse repetition frequency (PRF), which changes with the volume cover pattern (VCP).

The timestamps for these files were unevenly spaced on roughly five minute intervals. For some dates an unequal number of files were generated for the different parameters. For instance, on January 29, 2013, the process produced 133 files for Z, 11 files for $Z_{D R}$, and 26 files for $K_{D P}$. This limited the methods available for evaluation on dates where it occurred. The discrepancy was caused by variations in the VCP during the course of the event.

The radar files were zipped NetCDF files. Once unzipped, a regional subsection of the file was written in text format using the ncks tool in the NCO toolbox. Details on how this process was conducted are found in Appendix A. The subsection was chosen to completely encompass the locations of the rain gauges, as well as much of the surrounding area, to enable the trace calculations. The final subsection consisted of 44 
grid squares in latitude by 36 grid squares in longitude. Through a VBA script, the data found in the text files were transported to their final storage location within the Excel file where the main program was to be executed from.

\subsubsection{Timing}

All times either originated in UTC or were converted to UTC. Time calculations were all based on hours since start of event. Hour 0 for each event was assumed to be 00 UTC on the starting day. For example, hour 0 for the April 13 through April 15, 2012, event would be equivalent to 00 UTC on April 13. Hour 30.5 for the same event would be equivalent to 0630 UTC on April 14.

All rainfall estimation techniques were evaluated based on the five minute intervals the rain gauge data was recorded in. Through simple addition, 10 minute, 20 minute, 30 minute, and 60 minute intervals were also calculated. This made possible an estimation of effectiveness based on interval length, although only the 60 minute data would be analyzed in the study.

\subsubsection{Conventional Formula Interpolation}

Since the radar data were obtained at roughly (uneven) five minute intervals, it was necessary to interpolate values in between those times. Procedures for rainfall calculation depended on the technique used. For every technique, each time and site location was given a rainfall rate. To calculate rainfall for the five minute interval, the initial and final rainfall rates were averaged. For techniques only dependent on $\mathrm{Z}$ and/or $Z_{D R}$, or just $K_{D P}$, rain rates were calculated at the eight surrounding points (the two 
horizontal dimensions and time). The rainfall rate at the desired location and time was then found using linear interpolation. Figure 2.3 illustrates this process.

For techniques dependent on combinations of $\mathrm{K}_{D P}$ and $\mathrm{Z}_{\mathrm{DR}}, \mathrm{K}_{\mathrm{DP}}$ and $\mathrm{Z}$, or $\mathrm{K}_{\mathrm{DP}}, \mathrm{Z}$, and $\mathrm{Z}_{\mathrm{DR}}$, alterations in the calculations were necessary. This was due to the timing differences between the $\mathrm{Z}$ and $\mathrm{Z}_{\mathrm{DR}}$ radar data and the $\mathrm{K}_{\mathrm{DP}}$ data. For these combinations, $\mathrm{Z}$ and/or $\mathrm{Z}_{\mathrm{DR}}$ values at the eight surrounding points were converted to linear units, and values at the desired time were found via linear interpolation ( $\mathrm{K}_{\mathrm{DP}}$ values were also interpolated this way, although without conversion to linear units). Rainfall rates were then calculated at the resulting four points. Finally, the rainfall rate at the desired location was found through further linear interpolation.

The trace techniques dependent on model data were conducted slightly differently than the conventional methods. A detailed explanation is given in the Trace Method section. 

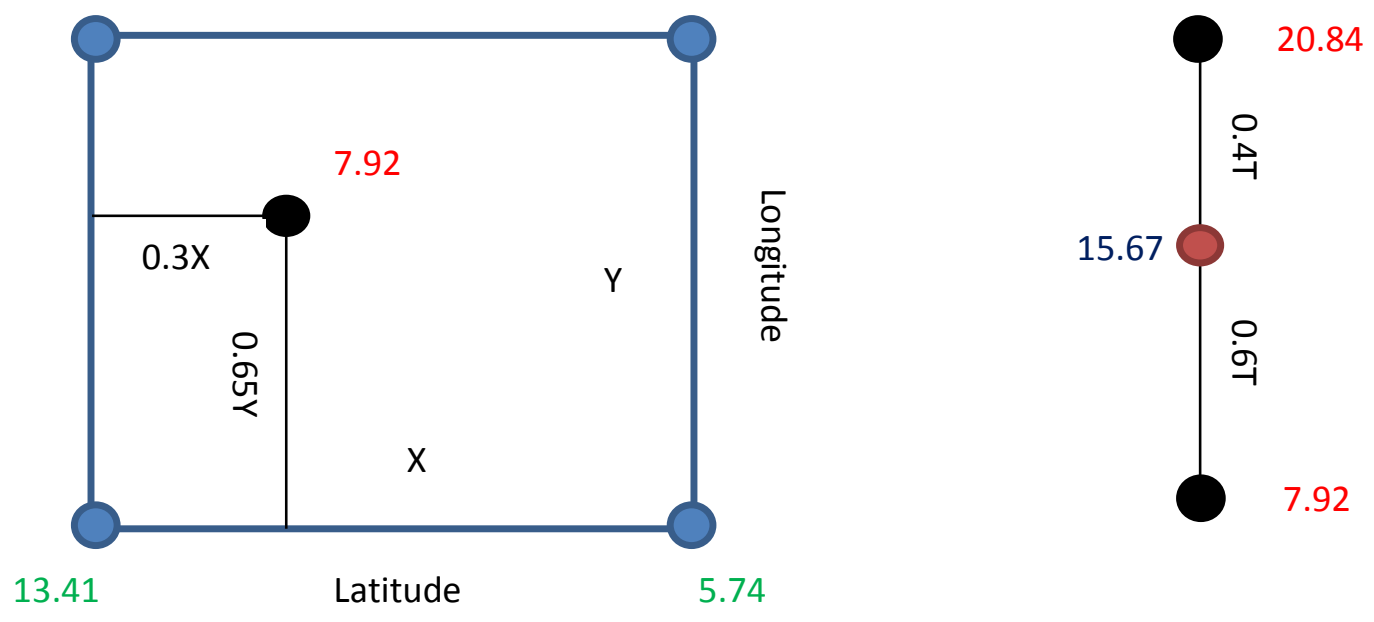

Figure 2.3. Interpolation Process for Formulas not Dependent on KDP and Another Parameter. In the top left image, the blue circles correspond to the surrounding four points where radar data is available. The black circle is the location of the point of interest. The green numbers are the rainfall rate calculations at the surrounding points in millimeters per hour. The red number is the rainfall rate (in $\mathrm{mm} /$ hour) at the point of interest, linearly interpolated from the four surrounding points.

The process shown in the top left image is performed twice, once for the radar image preceding the time of interest, and once for the radar image following the time of interest. In the top right image, the two red numbers correspond to these two calculated rainfall rates. The time of interest is marked by the red circle, and the blue number to the left is the linear interpolation of the rainfall rate calculated for the time of interest. 


\subsection{High Resolution Model Data and the Trace Method}

\subsubsection{Drift and Evaporation}

Traditional techniques for radar rainfall estimation assume the rain drops generating the observed reflectivity are instantly hitting the ground. As the radar beam rises with height, the assumption made in these techniques becomes worse as the point of interest increases in distance from the radar. Central Missouri is one such location, with the nearest radar being KLSX, located in Saint Louis.

Several factors affect where and at what size rain drops hit the surface. Given the necessary information horizontal drift and evaporation can be calculated. This is where high resolution model data becomes useful.

\subsubsection{Model Data}

The incorporation of high resolution model data into the rainfall estimation program required several steps. The data was obtained from NCDC. 13 kilometer resolution model data were obtained at hourly intervals. Data for the events preceding May of 2012 were from the Rapid Update Cycle (RUC) numerical weather model, which was replaced on May 1, 2012, by the Rapid Refresh (RAP). Both the RUC and the RAP issue hourly forecasts for a multitude of parameters across much of North America. This project used the initialization data for these models (forecast for hour zero). Height, temperature, relative humidity, $u$ wind, and $v$ wind were the necessary fields. Data was obtained at pressure levels ranging from 1000 millibars to 700 millibars (25 millibar intervals). 
The model data available from NCDC was in .grb2 format. The wgrib2 tool was used to convert the .grb2 files into csv files (comma separated variables). Detailed information on the use of the wgrib2 tool is available in Appendix A. The csv files were subsequently imported into Excel. The grid the model data came formatted in differed significantly from the grid the radar merging process produced. To map the model grid into a usable grid format several algorithms were employed. The resulting grid contained model data at a resolution of 0.1 degrees longitude by 0.1 degrees latitude.

\subsubsection{Trace Method}

The theory behind the trace method is that with the right information, a rain drop reaching the surface can be traced backwards in time to its origin. In this case the drop's "origin" refers the time and location at which the drop interacted with the radar beam. Knowledge of atmospheric temperature, relative humidity, wind speed, and wind direction can be used to calculate where the drop's origin is, as well as how much it has evaporated during its time spent in motion.

\subsubsection{CAPPI}

The CAPPI, or Constant Altitude Plan Position Indicator, was the format used for radar data. With dual polarized radar, the CAPPI provides information about drop size distribution at a constant height in the atmosphere. The CAPPI height used for this project was two kilometers above radar level. The CAPPI was chosen to be at two kilometers due to the relative distance between Central Missouri and the radar in Saint 
Louis. Two kilometers above the radar level lies within the lower portion of the $0.5^{\circ}$ elevation beam at the KLSX-Columbia distance, which is roughly $140 \mathrm{~km}$.

\subsubsection{Calculating Drop Size Distribution}

Rain drops reaching the surface range in size from roughly 0 to $6.0 \mathrm{~mm}$ in diameter. This is usually given in equivalent spherical drop diameter, as the drops themselves become less spherical as they grow larger. The ratio of vertical diameter to horizontal diameter is called the axis ratio. The axis ratio shrinks as the equivalent spherical drop diameter increases. The axis ratio formula used for this study comes from Beard and Chuang's 1987 study, and is shown in Equation 2.1 (Beard and Chuang 1987).

$$
\begin{gathered}
a / b=1.0048+0.00057 * D-0.02628 * D^{2}+0.003682 * D^{3}- \\
0.0001677 * D^{4} .
\end{gathered}
$$

In Equation 2.1, D is the drop diameter in millimeters. Other drop shape formulas are available, but as mentioned in Chapter 1 no significant variation is found in the drop diameter range of interest.

Differentially reflectivity, or $Z_{D R}$, is a value provided by dually polarized radars. The differential reflectivity is defined by

$$
Z_{D R}=10 * \log _{10}\left(\frac{Z_{H}}{Z_{V}}\right)
$$

where $Z_{H}$ is the horizontal reflectivity and $Z_{V}$ is the vertical reflectivity.

From Equation 2.1, a drop size distribution resulting in an equivalent differential reflectivity can be estimated. As mentioned in Chapter 1, the gamma drop size 
distribution has been shown to correspond best to real world situations. This drop size distribution is given by

$$
n(D)=n_{0} * D^{\mu} * \exp (-\Lambda D)
$$

where $n(D)$ is the number of drops of diameter $D$ per meter cubed per drop diameter interval.

To obtain a drop size distribution from just two parameters, reflectivity and differential reflectivity, a relationship between $\mu$ and $\Lambda$ must be assumed. In their 2004 paper Brandes et al. found that the relationship shown in Equation 2.4 worked best (Brandes et al. 2004).

$$
\Lambda=0.0365 * \mu^{2}+0.735 * \mu+1.935
$$

Drop concentrations (per $\mathrm{m}^{3}$ ) at (discrete) $0.01 \mathrm{~mm}$ intervals were calculated based on the drop size distribution from Equation 2.4, assuming an $n_{0}$ value of 1000 . From these concentrations predicted $Z$ and $Z_{D R}$ values were obtained. The $Z_{D R}$ values were dependent only on $\mu$ (and in this case unique); as a consequence corresponding $Z_{D R}$ and $\mu$ values were mapped out. Since adjusting the value of $n_{0}$ altered the reflectivity without changing the differential reflectivity, a value of $n_{0}$ and $\mu$ could be found to match up with every possible $Z$ and $Z_{D R}$ combination. Figure 2.4 shows expected rainfall rate based on different combinations of $Z$ and $Z_{D R}$, given the assumptions made about $\mu$ and $\Lambda$. As a side exercise the coefficients from Equation 2.4 were allowed to vary and the effects on expected rainfall rate are shown in Figure 2.5, Figure 2.6, and Figure 2.7. The assumption was made in these calculations that the reflectivity was at a constant 40 
dBZ. However, the percent change in rainfall rate was unaffected by the reflectivity value assumed. 


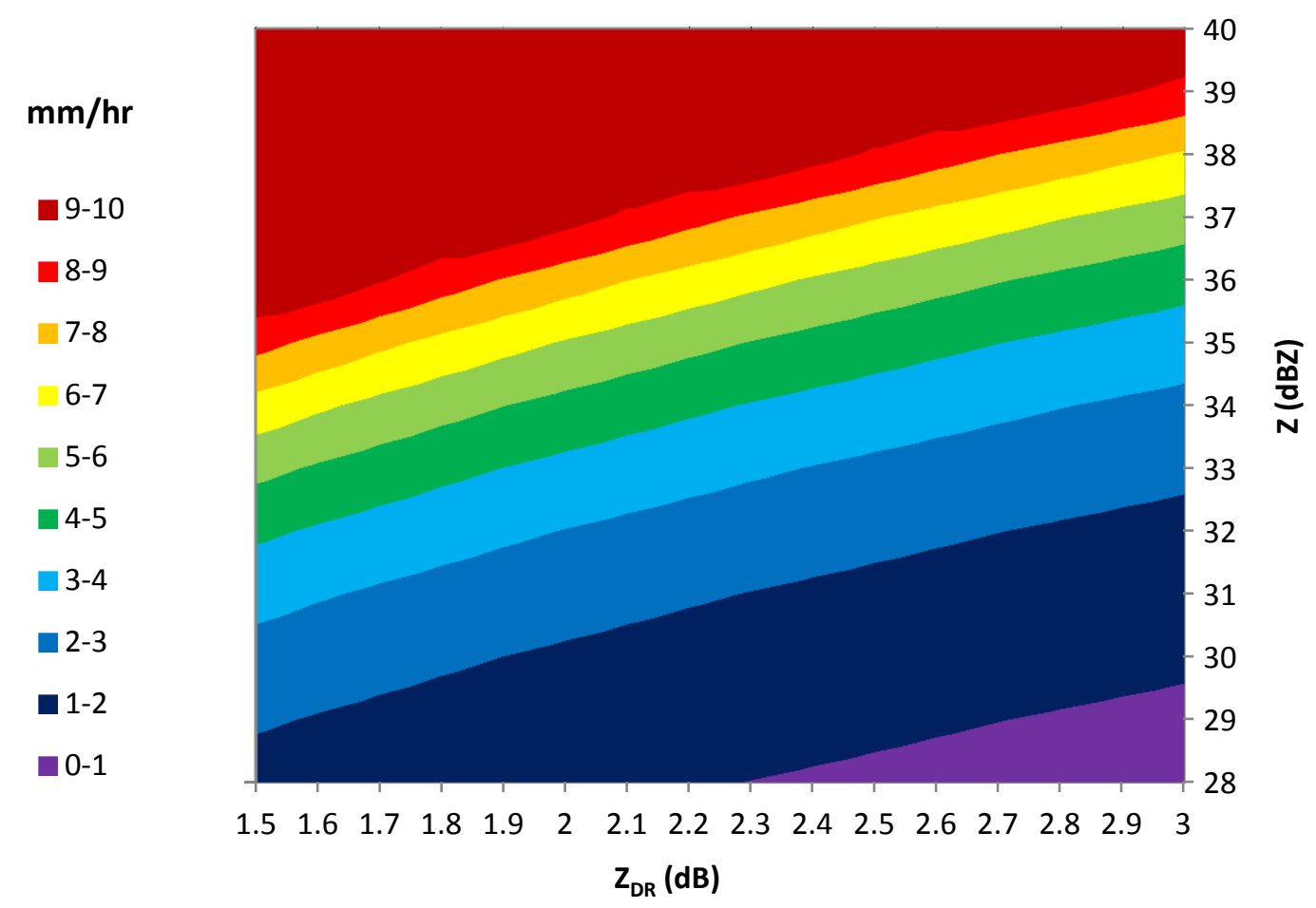

Figure 2.4. Theoretical Rainfall Rates with varying Z and ZDR ( $\mathrm{mm} /$ hour).

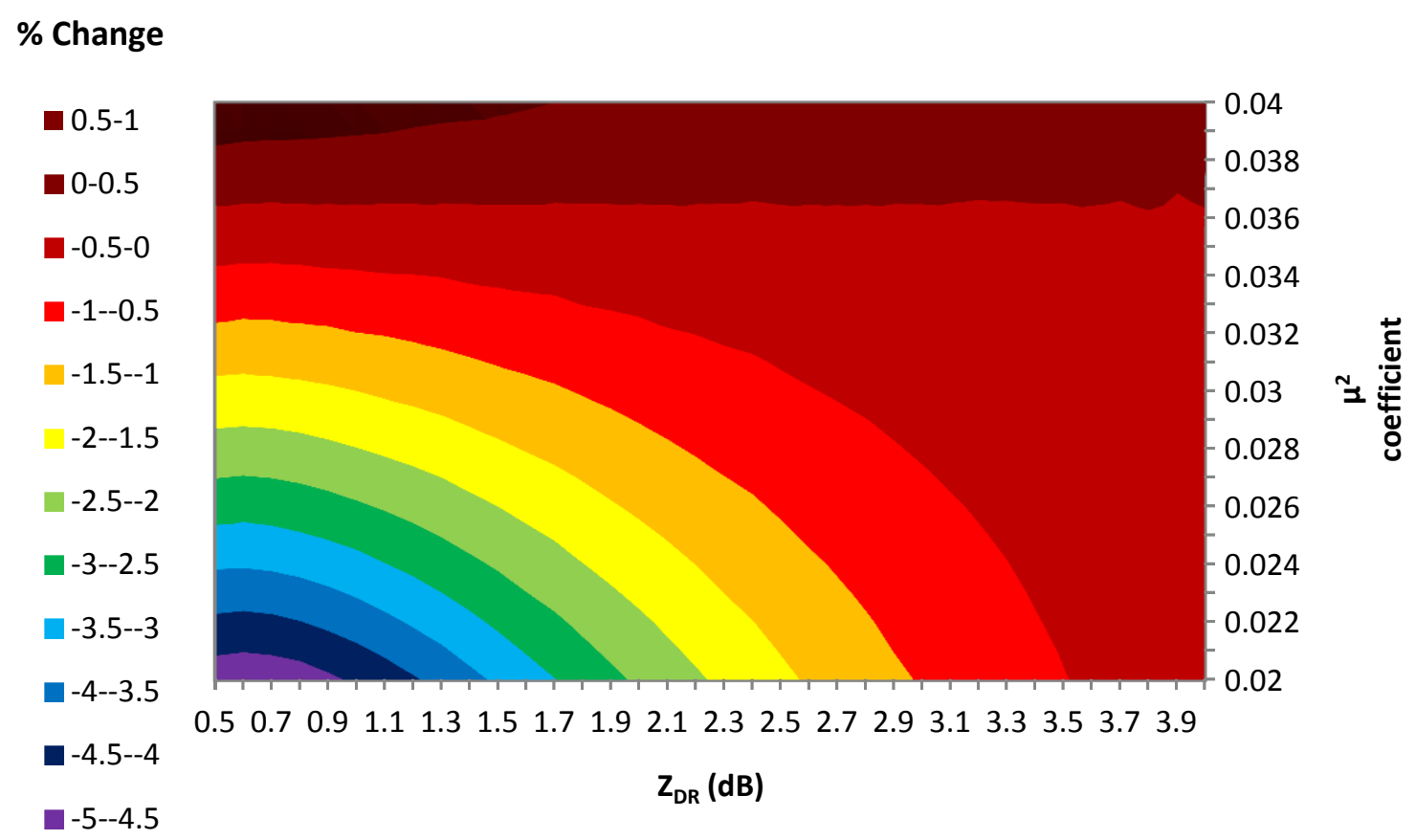

Figure 2.5. Change in Theoretical Rainfall Rate (\%) with Varying $Z_{D R}$ and $\mu^{2}$ Coefficient. 


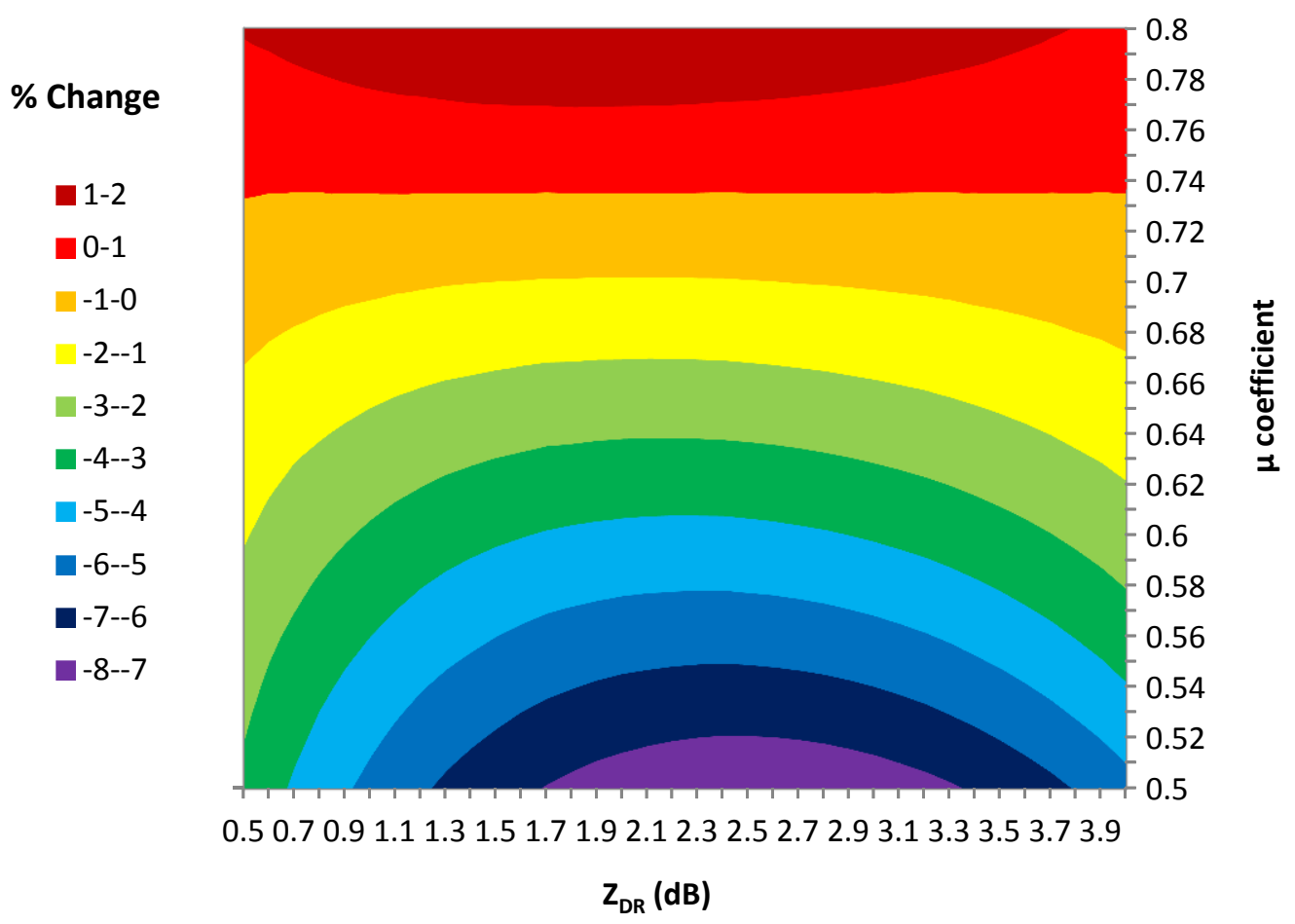

Figure 2.6. Change in Theoretical Rainfall Rate (\%) with Varying $Z_{D R}$ and $\mu$ Coefficient.

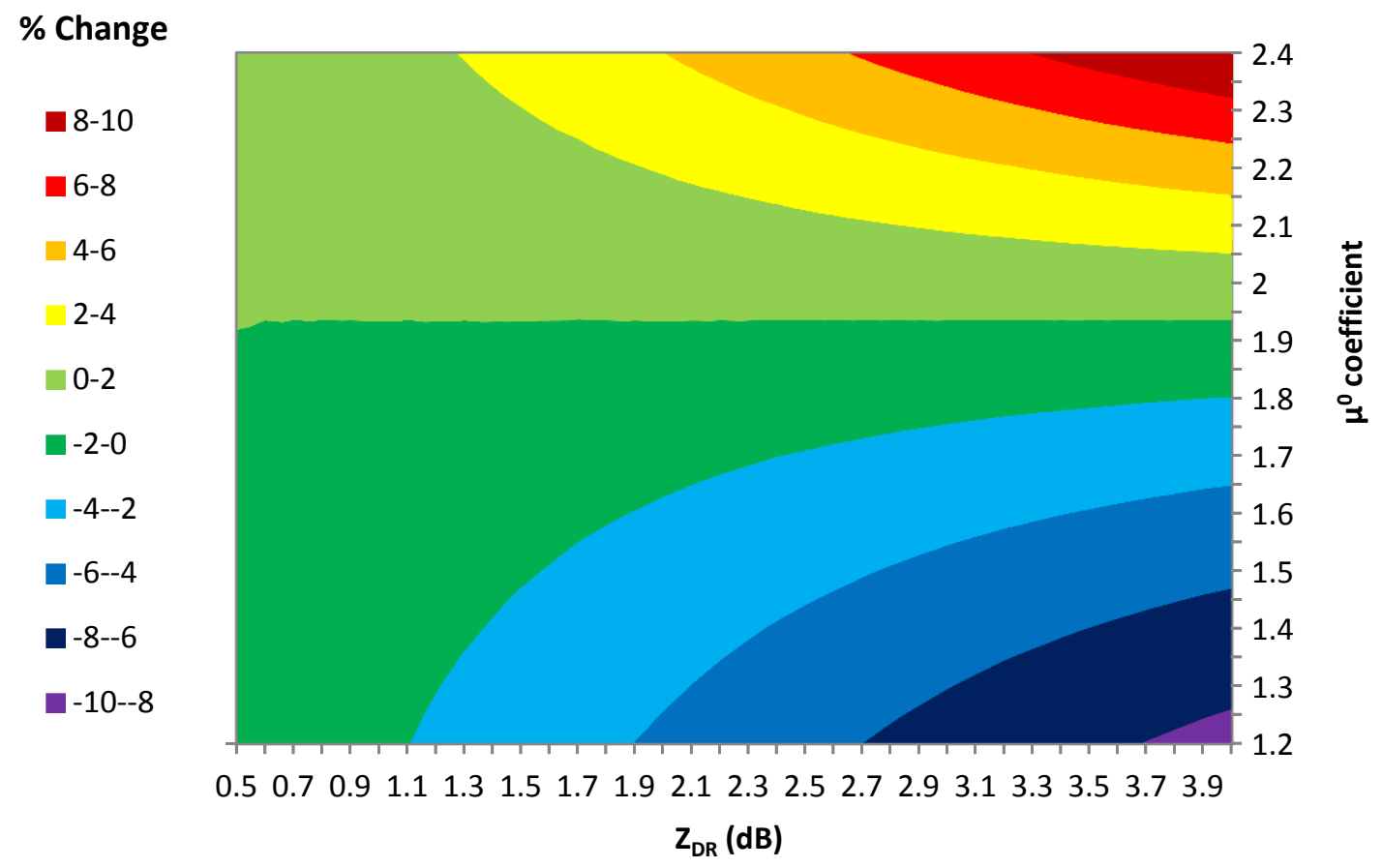

Figure 2.7. Change in Theoretical Rainfall rate (\%) with Varying $Z_{D R}$ and $\mu^{0}$ Coefficient. 


\subsubsection{Tracing the Drops}

At the surface, one might expect a certain concentration of drops of a particular size. To calculate a rainfall rate, a concentration for drops of all sizes is needed. The tracing process therefore attempts to trace drops of all sizes to their respective points of origin. However, at their origins these drops will be different sizes due to the evaporation process. As described in the literature review, drop evaporation is a process dependent on many factors, including drop size, environmental humidity, and environmental temperature. The equation used to calculate drop evaporation comes from $\mathrm{Li}$ and Srivastava's 2001 study and is shown in Equation 2.5 (Li and Srivastava 2001).

$$
\frac{d m}{d t}=4 \pi r D_{v} f_{v} \Delta \rho_{v}
$$

The ventilation coefficient, $f_{v}$, is calculated as

$$
f_{v}=0.78+0.308\left(\frac{v}{D_{v}}\right)^{\frac{1}{3}}\left(\frac{V D}{v}\right)^{\frac{1}{2}}
$$

In these equations, $m$ is the mass of the rain drop, $v$ is the kinematic viscosity of air, $V$ is the terminal velocity, $D_{v}$ is the diffusivity of water vapor in air, $r$ is the drop radius, $\mathrm{D}$ is the drop diameter, and $\Delta \rho_{v}$ is the difference in vapor density between the drop surface and the environment.

Along with evaporation, calculating drop terminal velocity is also necessary for tracing drops to their origin. Drop terminal velocity is also dependent on many factors, the most important being size and air density. The equation used for drop terminal velocity for this study was from Boxel's 1997 paper and is shown in Equation 2.7 (Boxel 1997). 


$$
V_{t}=\left(\frac{\rho_{b}}{\rho}\right)^{0.4} 9.65 *(1-\exp (-0.53 * D))
$$

For Equation $2.7 \rho_{b}$ is the standard atmosphere air density, $\rho$ is the air density at point of calculation, and $\mathrm{D}$ is the drop diameter in millimeters.

For each site, each day, and each five minute time step, 60 drop sizes were traced to their origins. The tracing was done in one second intervals. Every second the following parameters were recalculated for each individual drop.

- Latitude

- Longitude

- Height

- Pressure

- Relative humidity

- Temperature

- U wind

- $\quad V$ wind

- Terminal velocity

- Evaporation rate (mass loss per second)

- Virtual temperature

The three motion vectors given to each drop were used to calculate a position one second in the past. The evaporation rate was used to calculate a drop size for that position. New motion vectors and an evaporation rate were then calculated for the new 
position; these new values were averaged with the original values and used to calculate the drop's "official" position one second before. Calculation of the evaporation rate required estimation of the wet bulb temperature; this was done using an iterative process. Estimation of the pressure, which was needed for air density calculations, was done using the hypsometric equation and several assumptions about the temperature lapse rate. As described earlier, model data was obtained at 25 millibar intervals. The average virtual temperature of a layer is necessary to estimate its thickness. Given the virtual temperature at the top and bottom of the 25 millibar layer, an average lapse rate can be calculated. However, the average virtual temperature given by this average lapse rate may not necessarily correspond to the thickness of the layer given by the model data. To maintain the model estimated thickness of the layer, as well as the apparent average lapse rate in the model, the virtual temperatures at the top and bottom of the layer were adjusted. These virtual temperature assumptions, the predicted height of the drop, and the hypsometric equation were then used to calculate the pressure. A detailed explanation of this process is found in Appendix C.

A sample tracing was done for six rain drop sizes on August 31, 2012, and May 20, 2013. The drop diameters at the surface were $0.1,0.4,1.0,1.5,3.0$, and 6.0 millimeters. These drops reached the surface at site 1 .

On August 31, 2012, each drop was assumed to have reached the surface a few minutes after 12 UTC. Figure 2.8 shows the diameter of the four smallest drops as they are traced back to their points of origin, while Figure 2.9 shows the horizontal trajectories of 
all six drops. Figure 2.10 and Figure 2.11 show the same statistics for hypothetical drops on May 20, 2013. 


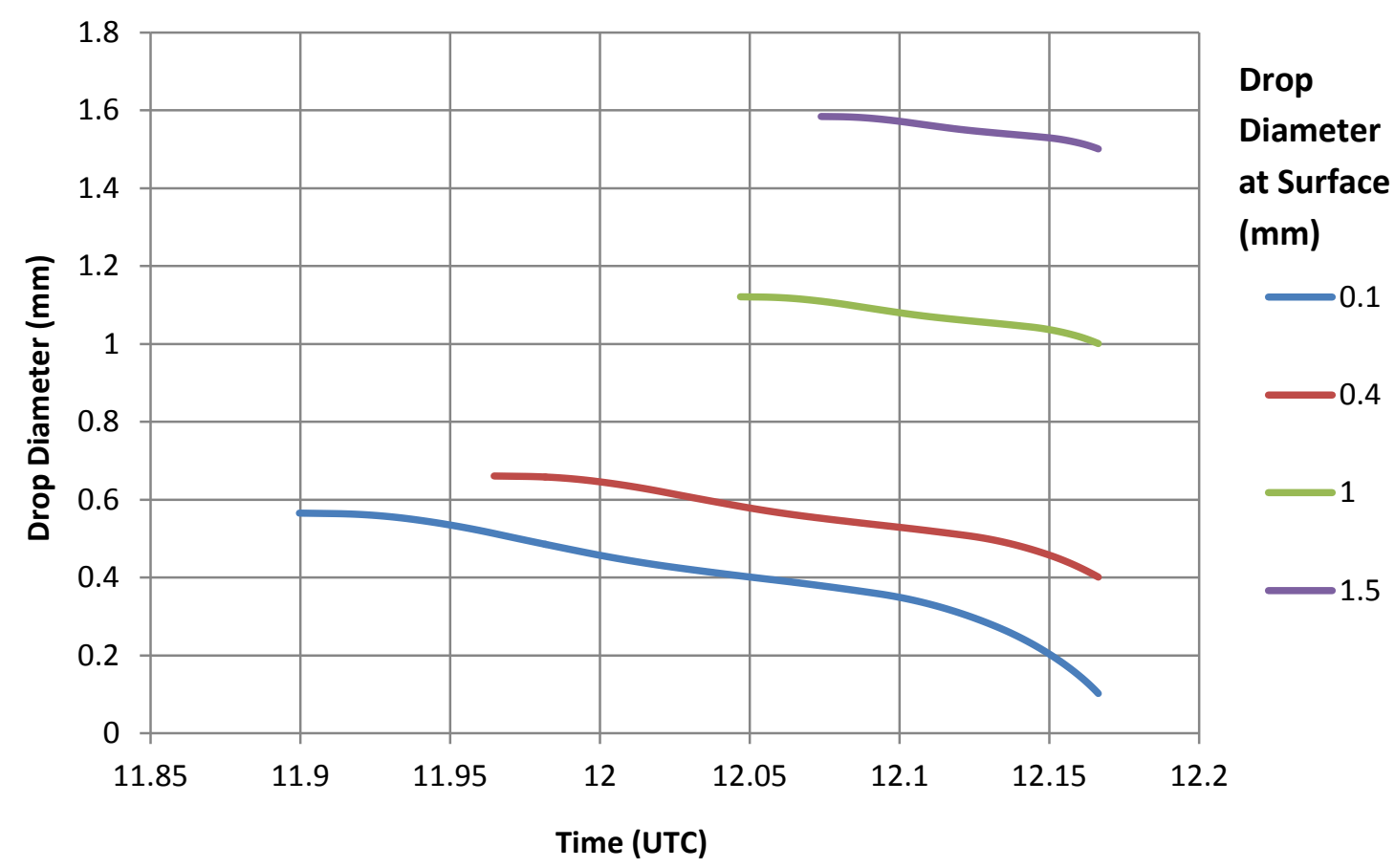

Figure 2.8. Drop Diameter as a Function of Time for August 31, 2012. Drop diameters at the surface are $0.1,0.4,1.0$, and $1.5 \mathrm{~mm}$. Drops reached the surface at roughly 1210 UTC.

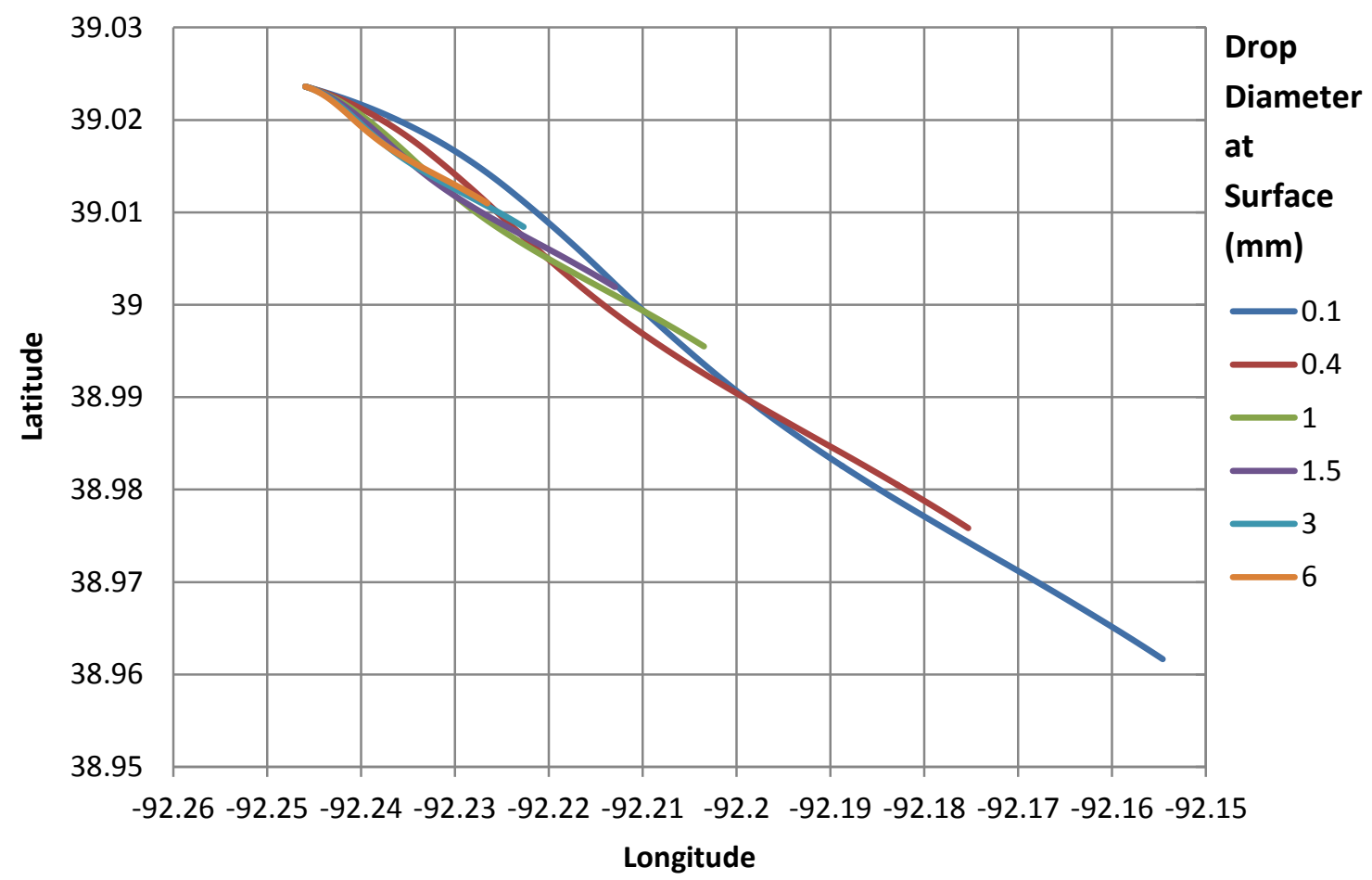

Figure 2.9. Drop Trajectories for August 31, 2012. Drop diameters at the surface are 0.1, 0.4, $1.0,1.5,3.0$, and $6.0 \mathrm{~mm}$. Drops reached the surface at $39.024^{\circ} \mathrm{N}, 92.246^{\circ} \mathrm{W}$. 


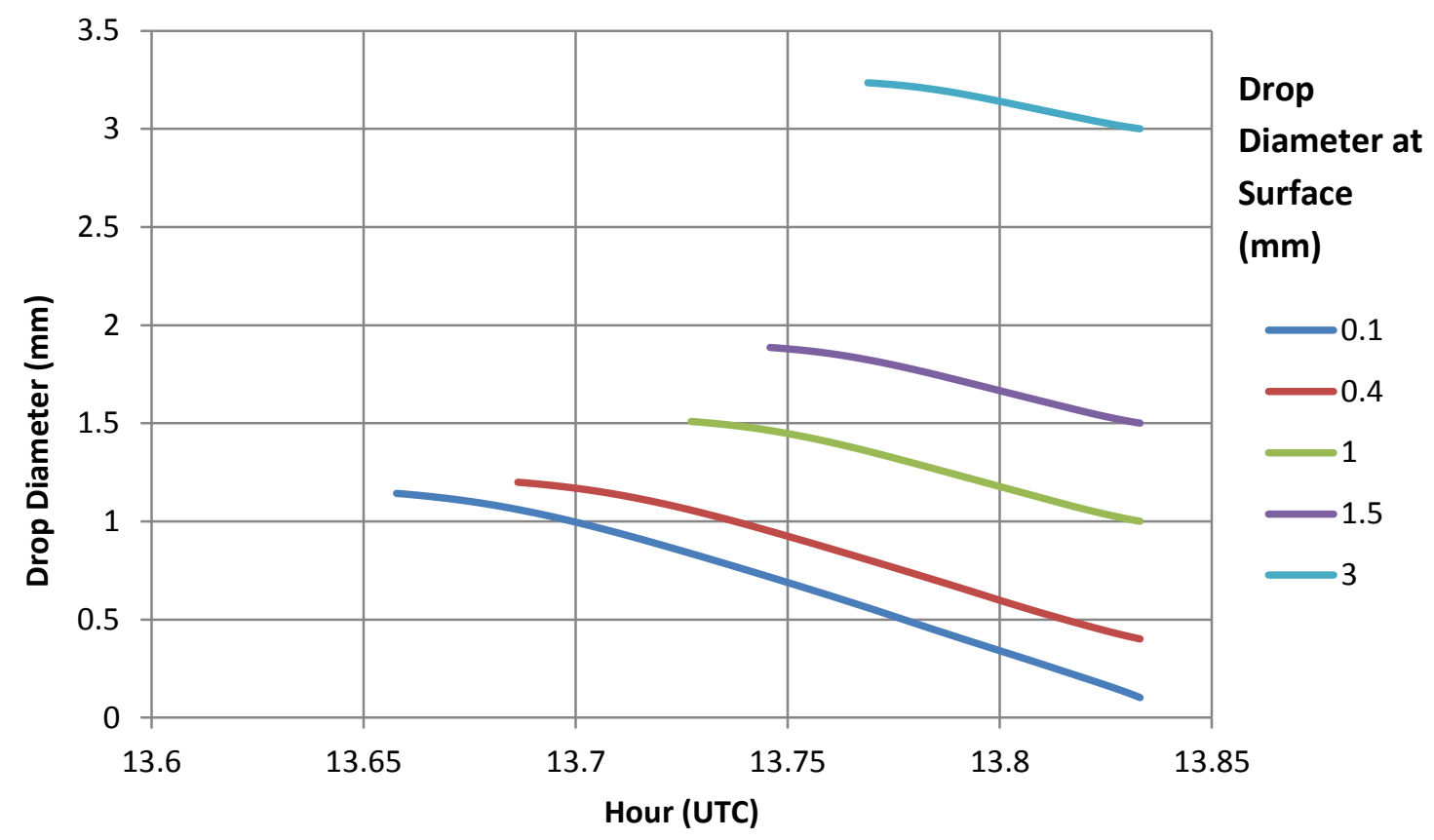

Figure 2.10. Drop Diameter as a Function of Time for May 20, 2013. Drop diameters at the surface are $0.1,0.4,1.0,1.5$, and $3.0 \mathrm{~mm}$. Drops reached the surface at roughly 1350 UTC.

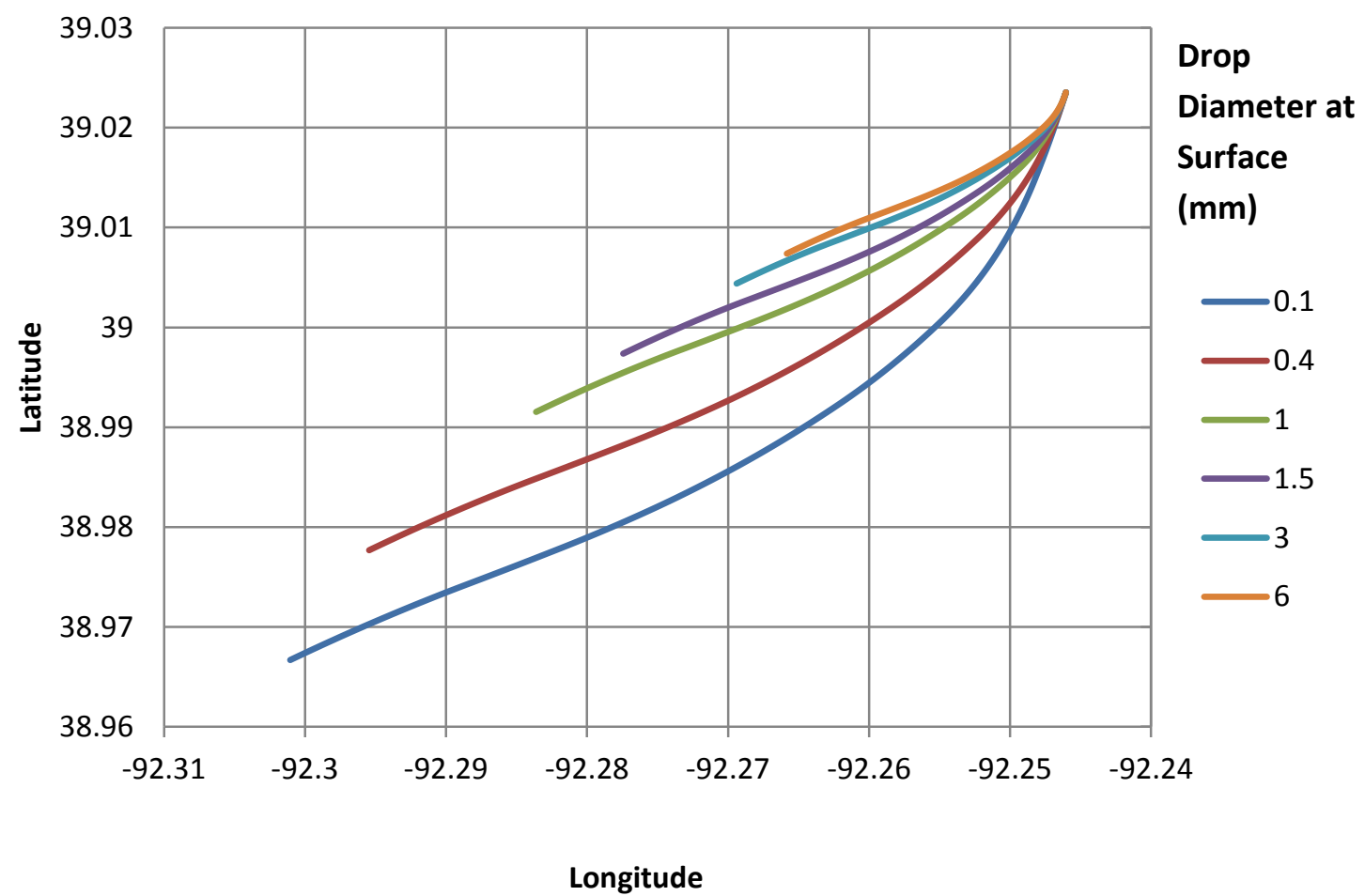

Figure 2.11. Drop Trajectories for May 20, 2013. Drop diameters at the surface are 0.1, 0.4, $1.0,1.5,3.0$, and $6.0 \mathrm{~mm}$. Drops reached the surface at $39.024^{\circ} \mathrm{N}, 92.246^{\circ} \mathrm{W}$. 


\subsubsection{Types of Tracing}

In addition to the tracing done to account for drift and evaporation, four other versions of drop tracing were also calculated for control purposes. The first was to simply assume the drops aloft instantly reached the ground with no drift or evaporation. The second was calculated under the assumption that the drops took time to reach the ground, but remained unaffected by drift or evaporation. The third and fourth versions took into account either drift or evaporation, but not both.

\subsubsection{Estimating Rainfall with the Trace Method}

For each five minute time step, the trace calculations yielded data on the origins of 60 drop sizes ranging from $0.1 \mathrm{~mm}$ in diameter to $6.0 \mathrm{~mm}$ in diameter (at $0.1 \mathrm{~mm}$ intervals). These drops were sorted by their size at the surface, as their size at point of origin varied considerably. Estimated points of origin were defined by three parameters: drop size at point of origin, time, and location.

For calculation of rainfall, a higher resolution of drop size was used. The drop sizes were further split into 591 categories, still ranging from $0.10 \mathrm{~mm}$ in diameter to $6.00 \mathrm{~mm}$ in diameter, but at $0.01 \mathrm{~mm}$ intervals. The points of origin for the drop sizes at $0.01 \mathrm{~mm}$ intervals were found through linear interpolation of the lower resolution points of origin. Figure 2.12 illustrates this process.

Once a time, size, size interval, and location of origin for a drop reaching the surface were obtained, the concentration of drops fitting those parameters was estimated using the drop size distribution derived from the corresponding radar data. Similar to the 
process shown in Figure 2.4, these concentrations were found at each of the eight surrounding points and interpolated linearly. The concentration of drops for each of the 591 drop sizes was obtained in this fashion, and their contribution to the overall rainfall rate was estimated and summed to generate the final rain rate at the time step in question. The rainfall rates at the beginning and end of each five minute interval were then averaged to determine the total rainfall during that period. 


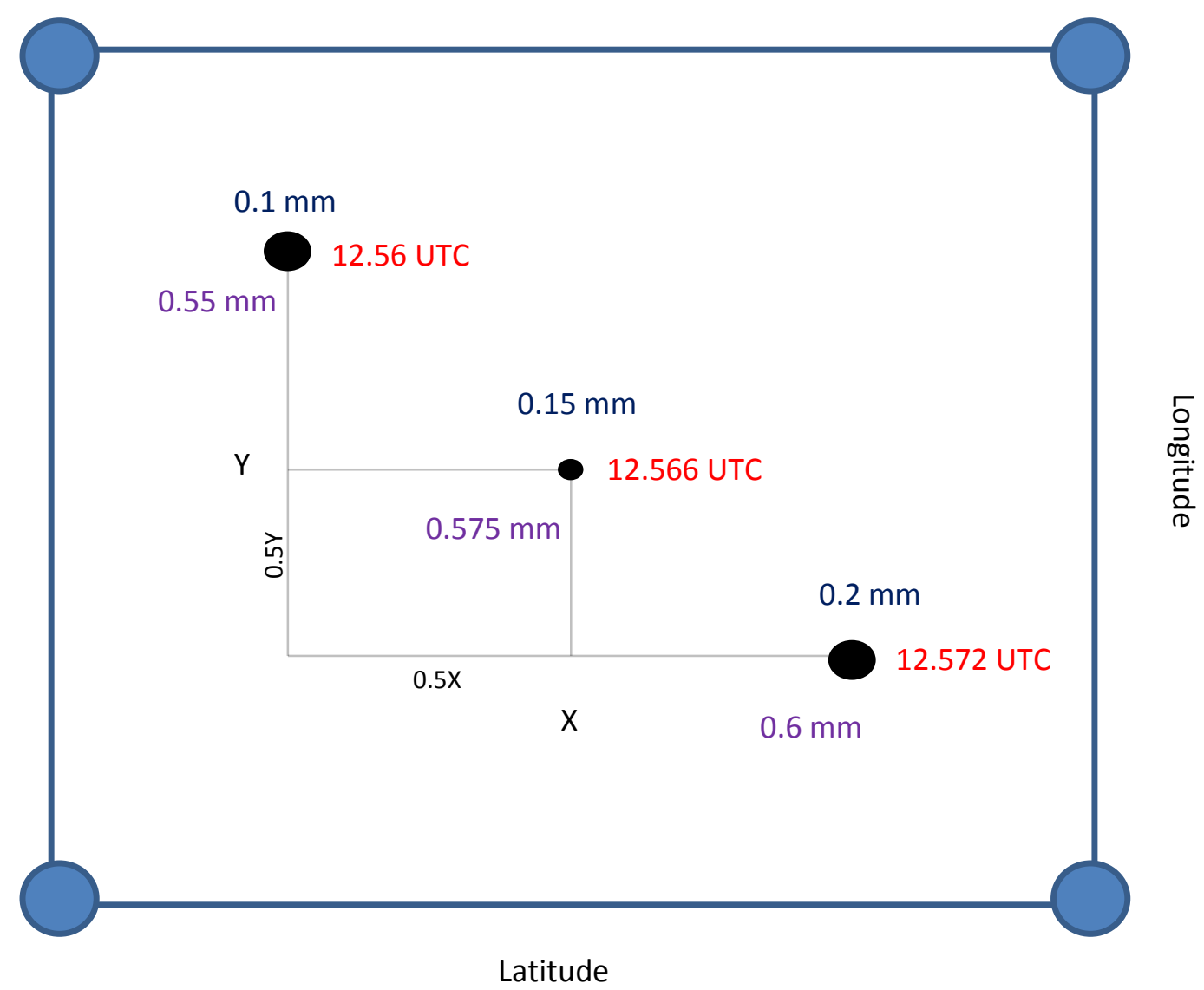

Figure 2.12. Illustration of the Drop Size Interpolation Process. The two larger black circles represent two hypothetical locations of origin for drops of $0.1 \mathrm{~mm}$ and $0.2 \mathrm{~mm}$ in diameter at the surface. Also shown for each drop is the time of origin in red and the drop size at point of origin in purple. $X$ is the longitudinal distance between the larger black circles and $Y$ is the latitudinal distance. The smaller black circle represents the interpolated location of origin for a drop of $0.15 \mathrm{~mm}$ in diameter at the surface; also present for the smaller black circle are the interpolated time and size information. The size interval for the $0.15 \mathrm{~mm}$ drop in this case would be $0.005 \mathrm{~mm}$. This means that for calculation purposes, drops between $0.5725 \mathrm{~mm}$ and $0.5775 \mathrm{~mm}$ in diameter at the point of origin are considered to all be $0.15 \mathrm{~mm}$ in diameter when they reach the surface. 


\subsection{Evaluated Techniques and Implementation}

\subsubsection{Evaluated Techniques}

Table 2.3 shows the conventional rainfall estimation techniques tested. Most of these techniques were also evaluated in RGS05. In addition to these formulas, the "synthetic" algorithm from RGS05 was also tested, albeit with slight alterations. The synthetic algorithm calculates rainfall rates using different formulas for different rain intensities. Equations 2.8-2.12 describe the synthetic algorithm.

$$
\begin{gathered}
R=\frac{\overline{R(Z)}}{f_{1}\left(\overline{Z_{d r}}\right)} \text { if } \overline{R(Z)}<6 m m r^{-1} \\
R=\frac{\overline{R\left(K_{D P}\right)}}{f_{2}\left(\overline{Z_{d r}}\right)} \text { if } 6<\overline{R(Z)}<50 m m r^{-1} \\
R=\overline{R\left(K_{D P}\right)} \text { if } 6<\overline{R(Z)}<50 m m h^{-1} \\
f_{1}\left(\left\langle Z_{d r}\right\rangle\right)=0.4+5.0\left|\left\langle Z_{d r}\right\rangle-1\right|^{1.3} \\
f_{2}\left(\left\langle Z_{d r}\right\rangle\right)=0.4+3.5\left|\left\langle Z_{d r}\right\rangle-1\right|^{1.7}
\end{gathered}
$$


In this case, $\left\langle Z_{d r}\right\rangle$ is the differential reflectivity in linear units. $\overline{R(Z)}$ is (2) from Table 1 and $\overline{R\left(K_{D P}\right)}$ is (9) from Table 2.3. The bar denotes mean values, using an averaging scheme described in RGS05. For this study, the only manipulation of $Z$ and $Z_{D R}$ was done in the merging process mentioned earlier. $K_{D P}$ values were smoothed using a simple filter that consisted of averaging the immediate surrounding cells with the cell in question.

As mentioned in Chapter 1, hail causes many problems when it comes to rainfall estimation using radar. A simple hail adjustment algorithm was implemented to reduce the effects of hail on the results, similar to the one described in the WSR-88D paper (Fulton et al. 1998). In this algorithm, reflectivity values were capped at $52 \mathrm{dBZ}$. A fuzzy logic scheme was implemented for reflectivity values between 45 and $52 \mathrm{dBZ}$. The fuzzy logic was designed to reduce reflectivity values for points where hail was more likely to be present, which in this case corresponded to $Z_{D R}$ values below the threshold of $3 \mathrm{~dB}$. $A$ detailed description of the fuzzy logic scheme is given in Appendix B.

Two other algorithms were used to improve the quality of the results. For the trace techniques, the minimum value allowed for $Z_{D R}$ was $0.5 \mathrm{~dB}$. This was implemented due to the range of $\mu$ values (Equation 2.4) utilized within the program. $\mu$ was allowed to vary between -5 and 16 , resulting in a $Z_{D R}$ range of 9.33 to 0.47 .

The last algorithm dealt with conventional formulas dependent on $\mathrm{K}_{\mathrm{DP}}$. This algorithm assumed a rain rate of zero for any location where reflectivity was below $20 \mathrm{dBZ}$, regardless of the $K_{D P}$ value. Implementation of this algorithm helped reduce noise within 
the $\mathrm{K}_{\mathrm{DP}}$-reliant formulas; applying the $20 \mathrm{dBZ}$ minimum provided assurance that the radar was actually picking up a significant signal at that location.

Table 2.3 Conventional Rainfall Formulas ( $\mathrm{R}$ in $\mathrm{mm} / \mathrm{hr}$ )

\begin{tabular}{|c|c|c|}
\hline$Z=a R^{b}$ & a & $b$ \\
\hline 1 & 200 & 1.6 \\
\hline 2 & 300 & 1.4 \\
\hline 3 & 250 & 1.2 \\
\hline 4 & 303 & 1.44 \\
\hline 5 & 527 & 1.41 \\
\hline$R\left(K_{D P}\right)=a\left|K_{D P}\right|^{b} \operatorname{sign}\left(K_{D P}\right)$ & a & b \\
\hline 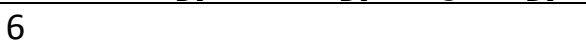 & 50.7 & 0.85 \\
\hline 7 & 54.3 & 0.806 \\
\hline 8 & 51.6 & 0.71 \\
\hline 9 & 44.0 & 0.822 \\
\hline 10 & 50.3 & 0.812 \\
\hline 11 & 47.3 & 0.791 \\
\hline
\end{tabular}

\begin{tabular}{lcccc} 
& $R\left(Z, Z_{D R}\right)=a Z^{b} Z_{d r}{ }^{c}$ & $\mathrm{a}$ & $\mathrm{b}$ & $\mathrm{c}$ \\
\hline 12 & $6.7 * 10^{-3}$ & 0.927 & -3.43 \\
13 & $7.46 * 10^{-3}$ & 0.945 & -4.76 \\
14 & $1.42 * 10^{-2}$ & 0.770 & -1.67 \\
15 & $1.59 * 10^{-2}$ & 0.737 & -1.03 \\
16 & $1.44 * 10^{-2}$ & 0.761 & -1.51 \\
\hline
\end{tabular}

\begin{tabular}{lccc}
$R\left(K_{D P}, Z_{D R}\right)=a\left|K_{D P}\right|^{b} Z_{d r}{ }^{c} \operatorname{sign}\left(K_{D P}\right)$ & $\mathrm{a}$ & $\mathrm{b}$ & $\mathrm{c}$ \\
\hline 17 & 90.8 & 0.93 & -1.69 \\
18 & 136 & 0.968 & -2.86 \\
19 & 52.9 & 0.852 & -0.53 \\
20 & 63.6 & 0.851 & -0.72 \\
\hline
\end{tabular}




\subsubsection{Trace Method and $Z_{D R}$ adjustments}

The five trace techniques evaluated are shown in Table 2.4. Also shown is the adjustments to $Z_{D R}$ that were tested. The reason for these adjustments was possible bias in $Z_{D R}$ values noted by the NWS (Hoban et al. 2014).

Table 2.4 Trace Techniques

\begin{tabular}{|c|c|c|}
\hline Description & Label & ZDR Adjustments \\
\hline Drift, Evaporation & T1 & $\begin{array}{l}+0,0.2,0.3,0.4,0.5,0.6,0.7 \\
0.8,0.9,1.0,1.1,1.5,2.0,2.6\end{array}$ \\
\hline $\begin{array}{l}\text { No Drift, No Evaporation, } \\
\text { Instant }\end{array}$ & $\mathrm{T} 2$ & $\begin{array}{l}+0.4,0.6,0.8,1.0,1.2,1.4,1.6 \\
1.8,2.0,2.2,2.4,2.6\end{array}$ \\
\hline No Drift, Evaporation & T3 & $\begin{array}{l}+0,0.2,0.3,0.4,0.5,0.6,0.7 \\
0.8,0.9,1.0,1.1,1.5,2.0,2.6\end{array}$ \\
\hline No Drift, No Evaporation & T4 & $\begin{array}{l}+0.4,0.6,0.8,1.0,1.2,1.4,1.6 \\
1.8,2.0,2.2,2.4,2.6\end{array}$ \\
\hline Drift, No Evaporation & T5 & $\begin{array}{l}+0.4,0.6,0.8,1.0,1.2,1.4,1.6 \\
1.8,2.0,2.2,2.4,2.6\end{array}$ \\
\hline
\end{tabular}




\subsubsection{Evaluation Methods}

The initial phase of program development focused on implementing conventional rainfall estimation techniques. These included simple Z-R relationships, and more complicated techniques that used combinations of $Z, Z_{D R}$, and $K_{D P}$. The next step involved implementing the calculation of parameters describing method effectiveness. The parameters chosen are listed in Table 2.5.

\section{Table 2.5 Evaluation Methods}

Evaluation Method Formula

Fractional Bias $\quad F B=\frac{\left\langle T_{R}-T_{G}\right\rangle}{\left\langle T_{G}\right\rangle}$

Fractional Absolute

Difference

$$
F A D=\frac{\left\langle\left|T_{R}-T_{G}\right|\right\rangle}{\left\langle T_{G}\right\rangle}
$$

Fractional Root Mean

Square Error

$$
F R M S E=\frac{\left\langle\left(T_{R}-T_{G}\right)^{2}\right\rangle^{1 / 2}}{\left\langle T_{G}\right\rangle}
$$

Fractional Standard

Deviation

$$
F S D=\left(F R M S E^{2}-F B^{2}\right)^{1 / 2}
$$

In Table $2.5 T_{R}$ is the total radar estimated rainfall, $T_{G}$ is the total gauge rainfall, and the brackets indicate averaging over all values. The pairs of corresponding rain gauge and radar estimated rainfall data had to fulfill two criteria before inclusion in the method effectiveness calculations. Double zero values were disqualified, as well as pairs where the rain gauge recorded zero accumulation and the radar estimated rainfall was at or 
below half the minimum possible rain gauge accumulation. For these rain gauges the minimum possible accumulation was 0.01 inches, or 0.254 millimeters.

The simplest graphical depiction of method effectiveness was an XY scatterplot with radar estimated rainfall on the $x$-axis and gauge rainfall on the $y$-axis. Figure 2.13 shows an example of this type of graphic.

In order to fulfill the "Flexibility" criterion, it was necessary to develop a control program. This program would take inputs like which formulas to use (and which constants to use with those formulas) and generate understandable results. A more detailed description of how this program functions is found in Appendix B.

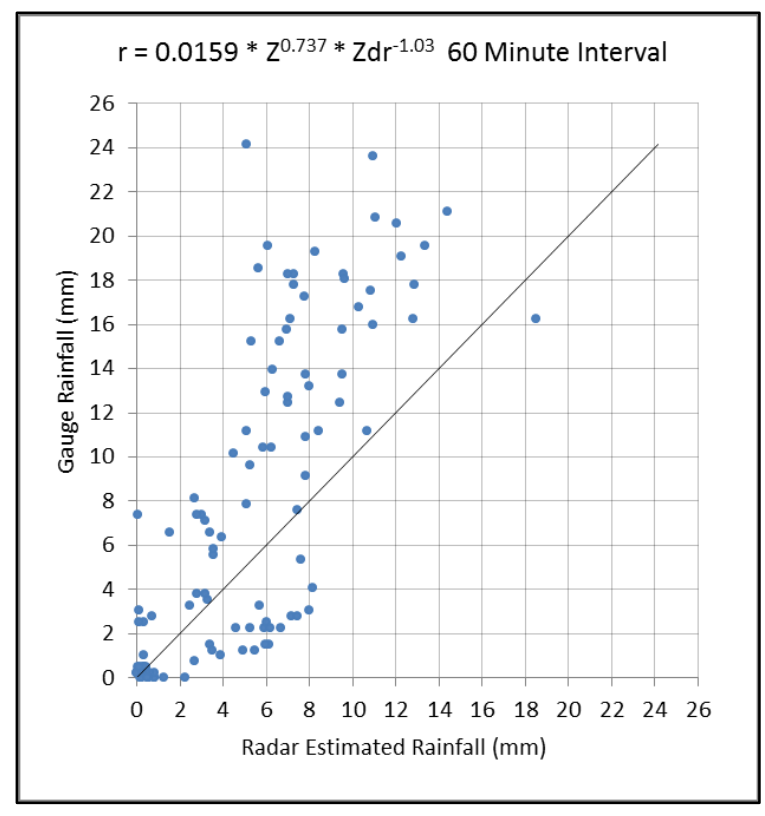

Figure 2.13. Sample X-Y Scatter plot of Radar Estimated Rainfall vs. Gauge Rainfall. The formula tested is shown in the top left corner. 


\section{Chapter 3. Results and Discussion}

\subsection{March 20, 2012}

Several competing air masses and at least two low pressure centers set the stage for widespread precipitation over the central United States. Figure 3.1.1 is an HPC surface analysis for 03 UTC of March 21, 2012. The precipitation occurred in several waves, beginning the evening of March 20 and continuing through the early morning hours.

Figure 3.1.2 shows humidity versus height in percentile format for 20 UTC of March 20 through 22 UTC of March 21; the data used for this figure came from one of the high resolution models. The percentiles are references to the amount of time spent at a particular relative humidity level. If the $50^{\text {th }}$ percentile showed relative humidity at $76 \%$, that would mean half of the event duration was spent above $76 \%$ relative humidity and half below it. A clear dry layer between 500 and 1000 meters above sea level is readily visible from Figure 3.1.2. Figure 3.1.3 is a hodograph also based on high resolution model data; the $U$ and $V$ values in Figure 3.1.3 are based on averages throughout the time period. The point nearest to the origin shows the $U$ and $V$ wind values at the 1000 millibar level; each subsequent point represents the $U$ and $V$ wind values at a pressure level 25 millibars above. On March 20, a persistent southeasterly wind was present from 1000 to 700 millibars. The direction changed to a more southerly orientation higher up 
in the atmosphere. The assumptions made in Figure 3.1.2 and Figure 3.1.3 were also made for the corresponding figures in other events.

\subsubsection{Conventional Techniques}

Table 3.1.1 shows the performance of the conventional techniques for this event. Of the conventional techniques, the most basic Z-R relationship, (1), proved to be the most effective. The parameters most used to describe method effectiveness were FAD and FRMSE. The lower these values were, the better the method performed. The formulas based on solely $Z$ were roughly equal to those based on $Z$ and $Z_{D R}$ for these parameters. A general negative bias was present for these types of formulas.

The $K_{D P}$ based formulas performed very poorly, as they did for most of the events. Some of this error is undoubtedly due to the general noisiness of $K_{D P}$ data. The method used to calculate $K_{D P}$ from $\phi_{D P}$ is another probable source of error. All the formulas using $K_{D P}$ exhibited a positive bias, with the Synthetic formula being the most accurate for every parameter.

\subsubsection{Tracing Techniques}

Table 3.1.2 shows the performance of the various trace techniques and their permutations for this event. The general pattern for the tracing techniques was that an increase in $Z_{D R}$ values resulted in reduced bias values. This makes sense due to the larger drops implied by larger $Z_{D R}$ values. These larger drops produce higher reflectivity values for the same quantity of water in the atmosphere, and as a consequence reduce the total estimated rainfall. 
Techniques T1 and T3 accounted for evaporation in their trace calculations. These techniques performed best in the +0.5 to $+0.8 \mathrm{Z}_{\mathrm{DR}}$ range. The other techniques performed best in the +1.2 to $+1.4 Z_{D R}$ range. It can be reasonably implied that for this date the evaporation that took place was roughly equivalent to $a+0.6$ to +0.7 increase in $Z_{D R}$.

If the events studied have a significant variation in the amount of evaporation occurring, the overall performance of the T2, T4, and T5 techniques at each individual category of $Z_{D R}$ adjustment will be reduced.

Out of all the techniques evaluated, the effectiveness of the $\mathrm{T} 3+0.6 \mathrm{Z}_{\mathrm{DR}}$ was best for the FAD and FRMSE parameters.

\subsubsection{Graphical Evaluation}

Figure 3.1.4 is a histogram showing the FAD performance of each evaluated technique for this event. Figure 3.1.5 is the equivalent histogram showing FRMSE performance. For these and subsequent histograms, the techniques are grouped into six sections. The conventional formulas are the first group, and make up the 21 separate techniques on the left edge of each histogram. The number of the technique corresponds to the number given in Table 2.3. These conventional formulas are further sorted by color. The green bars represent the simple Z-R relations, the red bars are the $K_{D P}-R$ relations, the dark blue bars are the $Z, Z_{D R}-R$ relations, the purple bars are the $K_{D P}, Z_{D R}-R$ relations, and the brown bar is the synthetic formula. The five other sections of techniques are the 
trace techniques, all light blue in color. Within each trace section are the varying ZDR adjustments used for that particular technique from Table 2.4.

Figure 3.1.6 shows a combination of scatterplots of radar-derived rainfall versus rain gauge rainfall for the conventional formulas. The techniques illustrated were the top performers in each category. Figure 3.1.7 is identical to 3.1.6, except it depicts the best performers of the trace techniques. These types of charts can be used to make a qualitative assessment of method performance, rather than quantitative. 


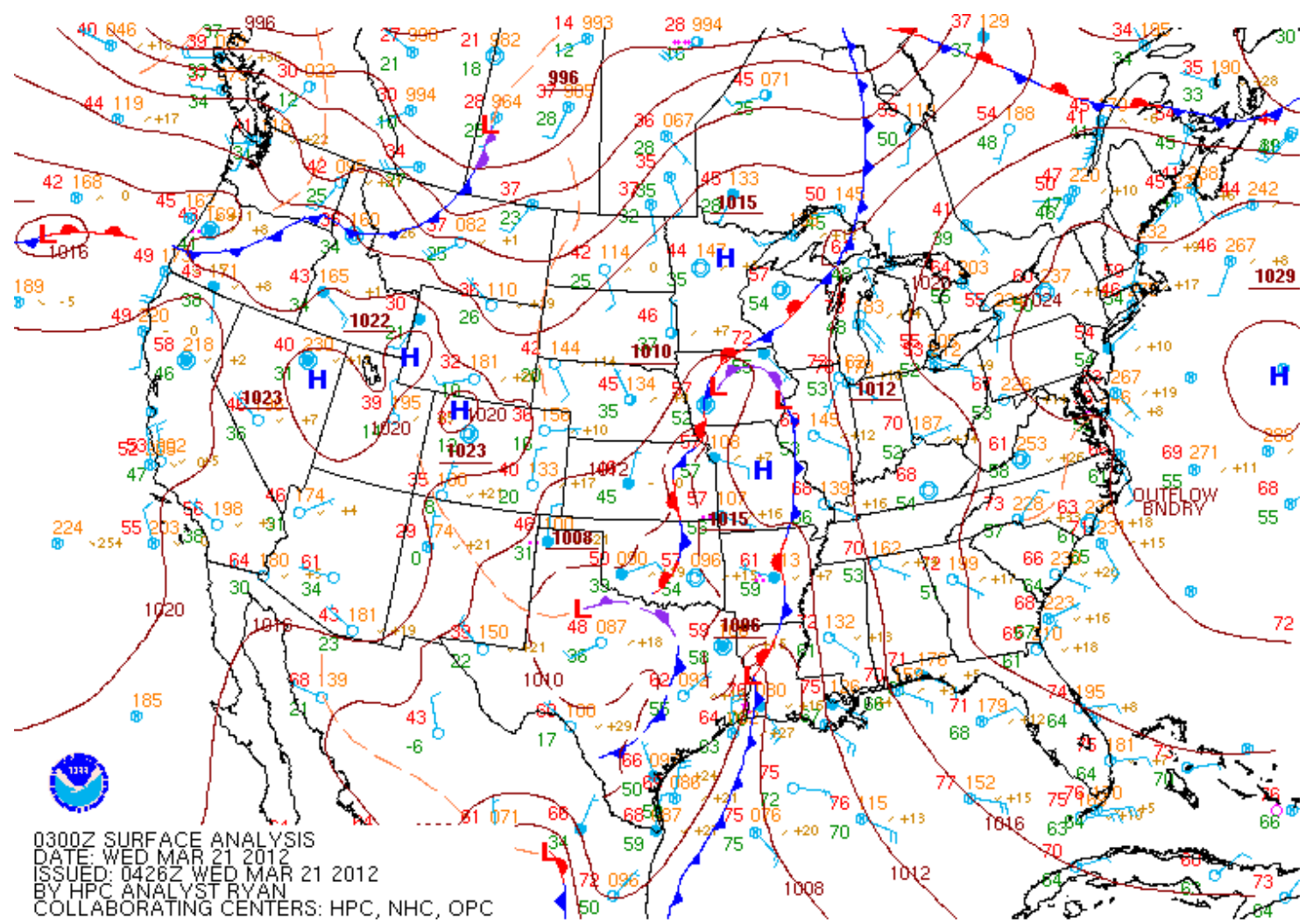

Figure 3.1.1. Surface Analysis 0300 UTC March 21, 2012.

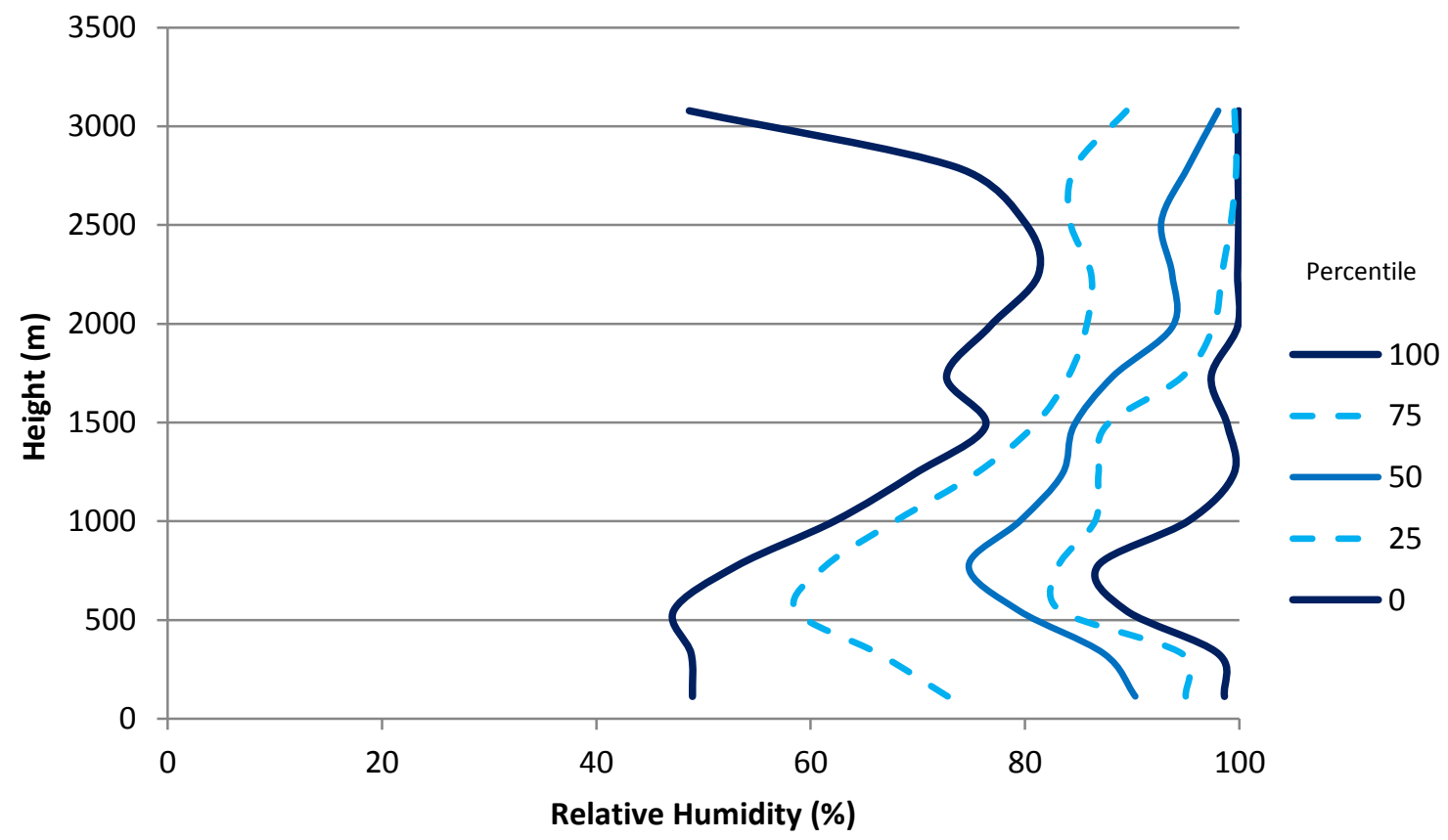

Figure 3.1.2. Relative Humidity Profile March 20, 2012. 


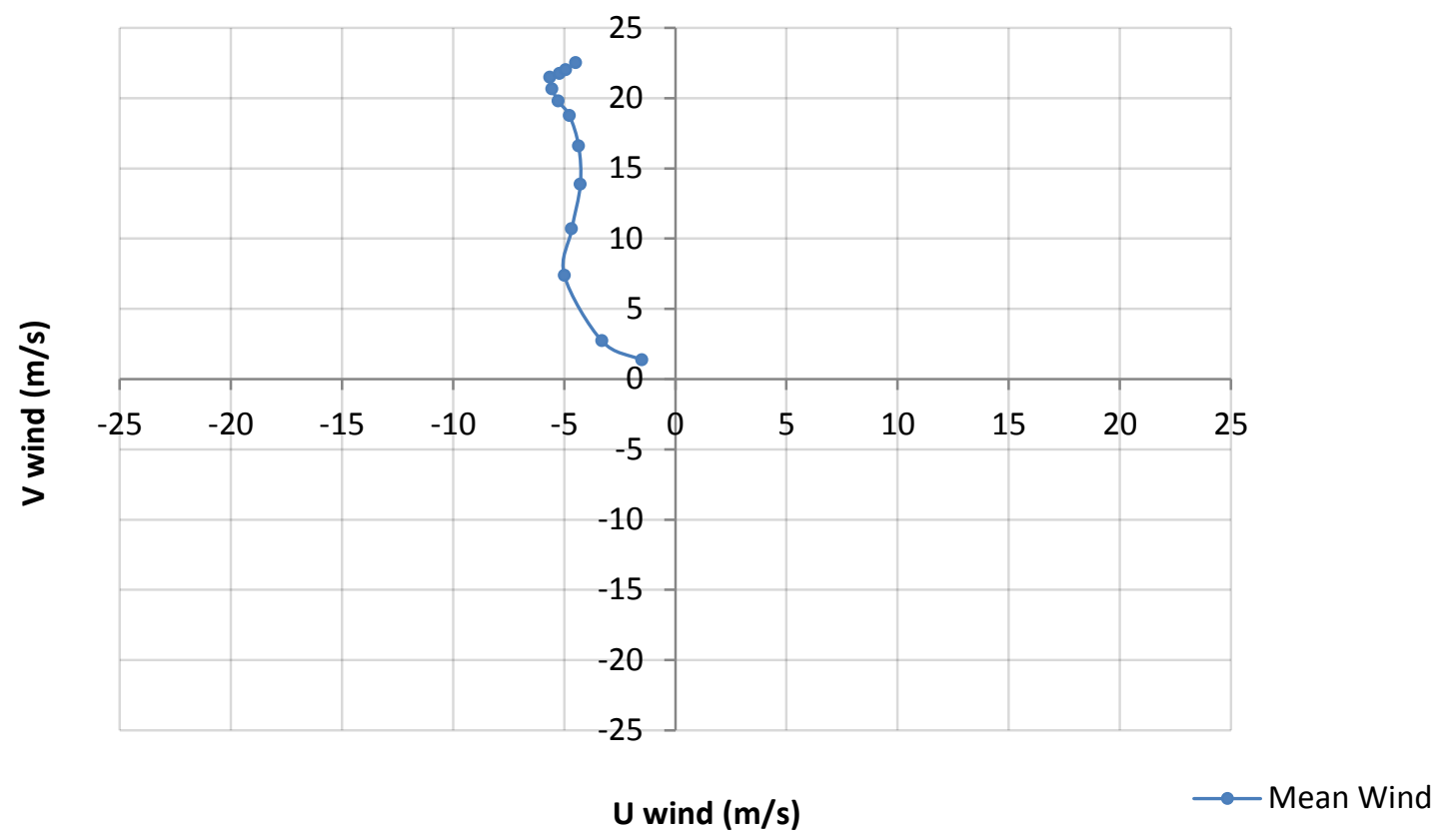

Figure 3.1.3. Hodograph March 20, 2012. 
Table 3.1.1 Conventional Techniques, March 20, 2012

\begin{tabular}{|c|c|c|c|c|c|}
\hline$Z=a R^{b}$ & $\#$ & $\mathrm{FB}(\%)$ & $\mathrm{FAD}(\%)$ & FRMSE(\%) & FSD(\%) \\
\hline 1 & 168 & -10.42 & 34.77 & 48.18 & 47.04 \\
\hline 2 & 166 & -19.69 & 35.65 & 50.89 & 46.93 \\
\hline 3 & 165 & 15.90 & 39.30 & 56.43 & 54.14 \\
\hline 4 & 166 & -22.87 & 37.47 & 53.87 & 48.78 \\
\hline 5 & 157 & -46.88 & 51.83 & 75.06 & 58.62 \\
\hline \multicolumn{6}{|c|}{$R\left(K_{D P}\right)=a\left|K_{D P}\right|^{b} \operatorname{sign}\left(K_{D P}\right)$} \\
\hline 6 & 175 & 63.67 & 148.58 & 203.00 & 192.75 \\
\hline 7 & 175 & 80.92 & 167.02 & 227.79 & 212.93 \\
\hline 8 & 175 & 84.56 & 172.29 & 232.90 & 217.01 \\
\hline 9 & 175 & 44.91 & 130.70 & 176.78 & 170.98 \\
\hline 10 & 175 & 66.86 & 152.08 & 207.31 & 196.23 \\
\hline 11 & 175 & 59.32 & 144.76 & 196.46 & 187.29 \\
\hline \multicolumn{6}{|c|}{$R\left(Z, Z_{D R}\right)=a Z^{b} Z_{d r}{ }^{c}$} \\
\hline 12 & 160 & -2.92 & 35.61 & 49.22 & 49.14 \\
\hline 13 & 162 & 7.33 & 39.40 & 57.55 & 57.08 \\
\hline 14 & 163 & -20.91 & 35.57 & 50.99 & 46.51 \\
\hline 15 & 164 & -24.11 & 37.44 & 53.92 & 48.23 \\
\hline 16 & 163 & -23.32 & 36.79 & 52.85 & 47.42 \\
\hline \multicolumn{6}{|c|}{$R\left(K_{D P}, Z_{D R}\right)=a\left|K_{D P}\right|^{b} Z_{d r}^{c} \operatorname{sign}\left(K_{D P}\right)$} \\
\hline 17 & 176 & 123.15 & 213.31 & 296.49 & 269.70 \\
\hline 18 & 176 & 178.68 & 277.75 & 390.90 & 347.67 \\
\hline 19 & 175 & 64.00 & 150.49 & 204.69 & 194.42 \\
\hline 20 & 175 & 92.19 & 180.03 & 246.18 & 228.27 \\
\hline \multicolumn{6}{|l|}{ Synthetic } \\
\hline 21 & 174 & 19.94 & 51.71 & 89.29 & 87.04 \\
\hline
\end{tabular}


Table 3.1.2 Trace Techniques, March 20, 2012

\begin{tabular}{|c|c|c|c|c|c|c|}
\hline & $Z_{D R}+$ & $\#$ & $\mathrm{FB}(\%)$ & FAD(\%) & FRMSE(\%) & $\mathrm{FSD}(\%)$ \\
\hline \multirow[t]{14}{*}{$\mathrm{T} 1$} & 0 & 151 & 17.25 & 43.85 & 77.94 & 76.01 \\
\hline & 0.2 & 152 & 8.51 & 39.63 & 67.03 & 66.49 \\
\hline & 0.3 & 152 & 3.72 & 38.17 & 62.25 & 62.14 \\
\hline & 0.4 & 152 & -1.10 & 37.77 & 58.35 & 58.34 \\
\hline & 0.5 & 151 & -5.93 & 37.75 & 55.30 & 54.98 \\
\hline & 0.6 & 151 & -10.58 & 38.12 & 53.63 & 52.57 \\
\hline & 0.7 & 150 & -15.26 & 38.32 & 52.40 & 50.13 \\
\hline & 0.8 & 150 & -19.77 & 38.84 & 52.22 & 48.34 \\
\hline & 0.9 & 149 & -24.13 & 39.50 & 52.61 & 46.75 \\
\hline & 1 & 149 & -28.24 & 40.64 & 53.82 & 45.81 \\
\hline & 1.1 & 149 & -32.17 & 41.98 & 55.59 & 45.33 \\
\hline & 1.5 & 149 & -45.39 & 48.61 & 65.62 & 47.39 \\
\hline & 2 & 149 & -57.26 & 58.63 & 78.36 & 53.49 \\
\hline & 2.6 & 149 & -67.19 & 67.74 & 90.55 & 60.70 \\
\hline \multirow[t]{12}{*}{ T2 } & 0.4 & 160 & 75.64 & 85.83 & 146.09 & 124.99 \\
\hline & 0.6 & 159 & 46.82 & 62.81 & 104.52 & 93.44 \\
\hline & 0.8 & 156 & 23.22 & 48.25 & 74.43 & 70.72 \\
\hline & 1 & 154 & 4.37 & 39.41 & 55.95 & 55.78 \\
\hline & 1.2 & 154 & -10.30 & 36.32 & 48.76 & 47.66 \\
\hline & 1.4 & 151 & -21.99 & 37.53 & 49.01 & 43.80 \\
\hline & 1.6 & 149 & -31.38 & 39.93 & 53.38 & 43.18 \\
\hline & 1.8 & 149 & -39.01 & 44.12 & 59.36 & 44.74 \\
\hline & 2 & 149 & -45.38 & 48.96 & 65.45 & 47.15 \\
\hline & 2.2 & 149 & -50.80 & 53.47 & 71.19 & 49.88 \\
\hline & 2.4 & 149 & -55.46 & 57.38 & 76.48 & 52.66 \\
\hline & 2.6 & 149 & -59.53 & 60.90 & 81.30 & 55.37 \\
\hline \multirow[t]{14}{*}{ T3 } & 0 & 150 & 10.88 & 41.28 & 63.57 & 62.63 \\
\hline & 0.2 & 150 & 2.69 & 37.49 & 54.80 & 54.73 \\
\hline & 0.3 & 150 & -1.85 & 36.08 & 50.84 & 50.80 \\
\hline & 0.4 & 150 & -6.52 & 35.03 & 47.89 & 47.44 \\
\hline & 0.5 & 150 & -11.19 & 34.41 & 46.14 & 44.77 \\
\hline & 0.6 & 150 & -15.78 & 34.38 & 45.53 & 42.71 \\
\hline & 0.7 & 150 & -20.28 & 34.60 & 46.02 & 41.31 \\
\hline & 0.8 & 149 & -24.63 & 35.51 & 47.29 & 40.37 \\
\hline & 0.9 & 149 & -28.71 & 37.00 & 49.43 & 40.24 \\
\hline & 1 & 149 & -32.56 & 38.72 & 52.06 & 40.62 \\
\hline & 1.1 & 149 & -36.17 & 41.02 & 54.97 & 41.39 \\
\hline & 1.5 & 149 & -48.36 & 51.04 & 67.09 & 46.51 \\
\hline & 2 & 149 & -59.46 & 60.68 & 80.21 & 53.83 \\
\hline & 2.6 & 149 & -68.86 & 69.28 & 92.22 & 61.34 \\
\hline
\end{tabular}


Table 3.1.2 Trace Techniques, March 20, 2012 (Continued)

\begin{tabular}{|c|c|c|c|c|c|c|}
\hline & $Z_{D R}+$ & $\#$ & $\mathrm{FB}(\%)$ & $\mathrm{FAD}(\%)$ & FRMSE(\%) & FSD(\%) \\
\hline \multirow[t]{12}{*}{ T4 } & 0.4 & 163 & 75.79 & 83.90 & 146.58 & 125.47 \\
\hline & 0.6 & 160 & 46.88 & 60.36 & 103.89 & 92.71 \\
\hline & 0.8 & 156 & 23.23 & 45.77 & 72.77 & 68.96 \\
\hline & 1 & 156 & 4.44 & 38.03 & 54.23 & 54.05 \\
\hline & 1.2 & 155 & -10.28 & 35.12 & 46.76 & 45.62 \\
\hline & 1.4 & 151 & -22.01 & 35.46 & 46.93 & 41.45 \\
\hline & 1.6 & 149 & -31.39 & 38.19 & 51.59 & 40.94 \\
\hline & 1.8 & 149 & -39.02 & 43.23 & 57.88 & 42.75 \\
\hline & 2 & 149 & -45.40 & 48.60 & 64.22 & 45.42 \\
\hline & 2.2 & 149 & -50.81 & 53.21 & 70.16 & 48.39 \\
\hline & 2.4 & 149 & -55.47 & 57.22 & 75.60 & 51.37 \\
\hline & 2.6 & 149 & -59.53 & 60.77 & 80.54 & 54.25 \\
\hline \multirow[t]{12}{*}{ T5 } & 0.4 & 158 & 86.88 & 94.92 & 170.38 & 146.57 \\
\hline & 0.6 & 156 & 57.17 & 71.01 & 127.77 & 114.27 \\
\hline & 0.8 & 156 & 32.26 & 53.83 & 94.91 & 89.26 \\
\hline & 1 & 155 & 12.26 & 43.89 & 71.06 & 70.00 \\
\hline & 1.2 & 152 & -3.87 & 39.19 & 56.41 & 56.28 \\
\hline & 1.4 & 152 & -16.48 & 38.52 & 51.78 & 49.09 \\
\hline & 1.6 & 151 & -26.64 & 39.84 & 53.09 & 45.92 \\
\hline & 1.8 & 151 & -34.91 & 42.91 & 57.53 & 45.74 \\
\hline & 2 & 151 & -41.76 & 46.37 & 62.99 & 47.16 \\
\hline & 2.2 & 151 & -47.58 & 50.52 & 68.58 & 49.38 \\
\hline & 2.4 & 149 & -52.65 & 54.64 & 73.41 & 51.16 \\
\hline & 2.6 & 149 & -56.98 & 58.41 & 78.33 & 53.75 \\
\hline
\end{tabular}




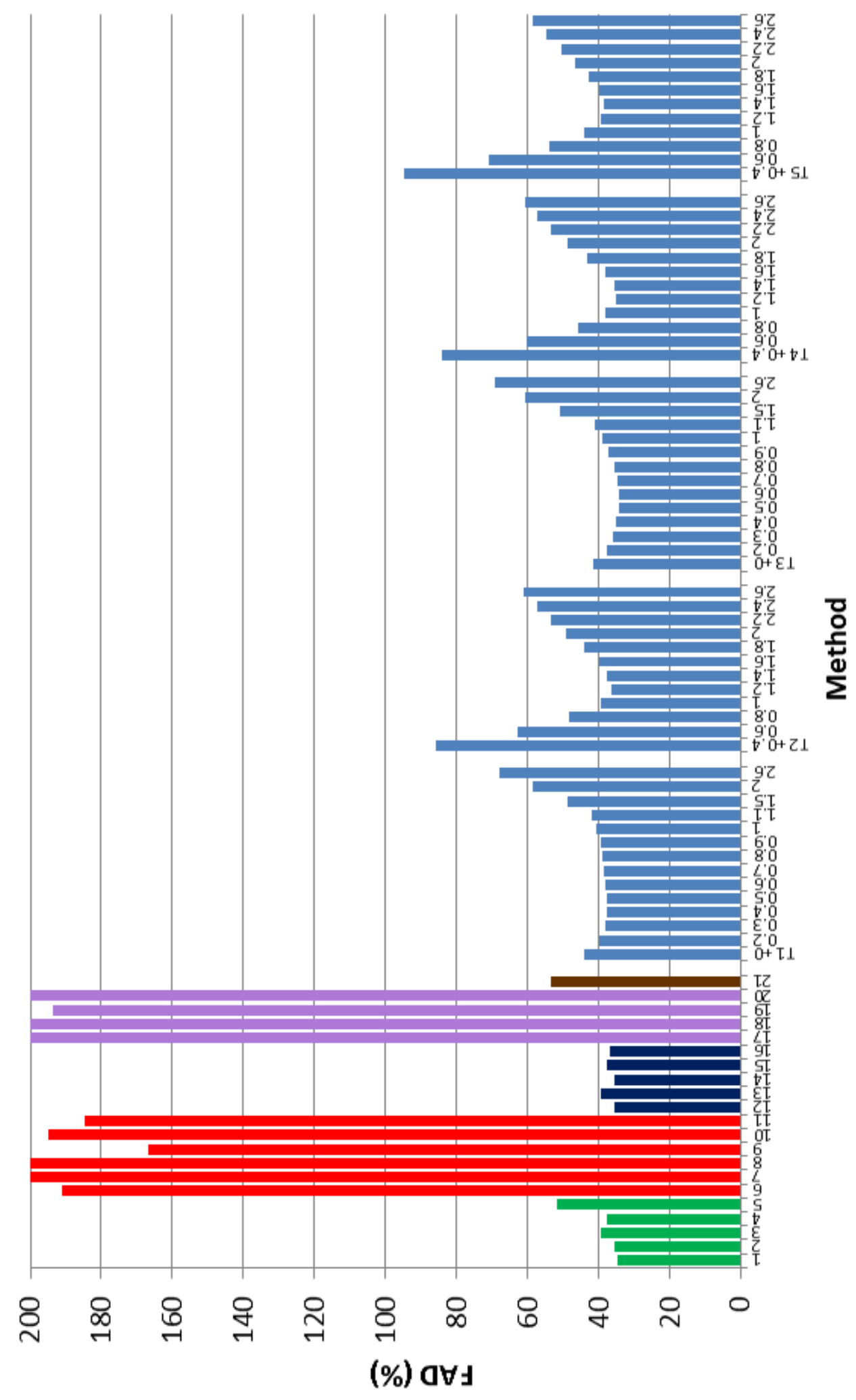

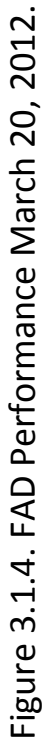




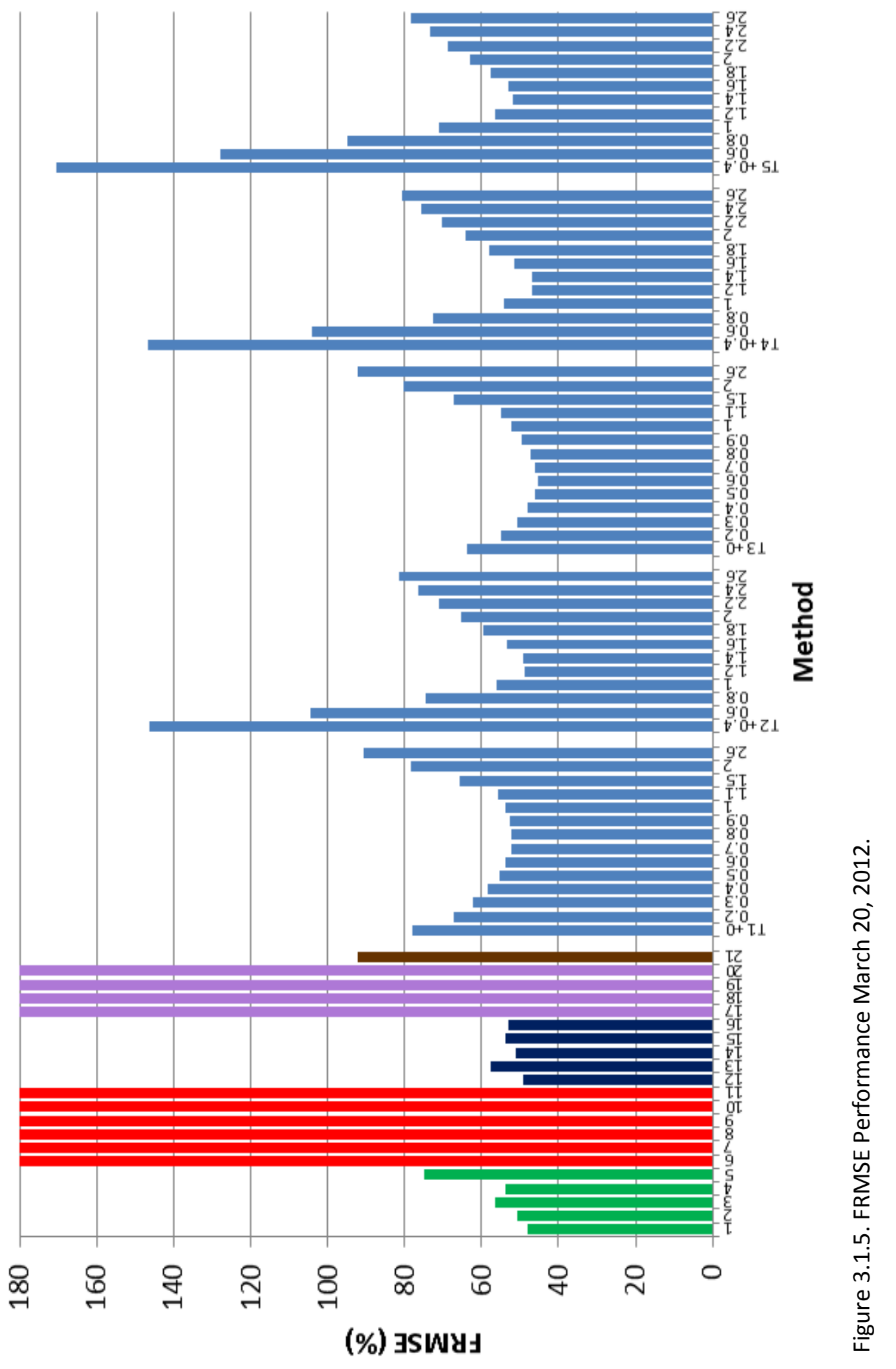




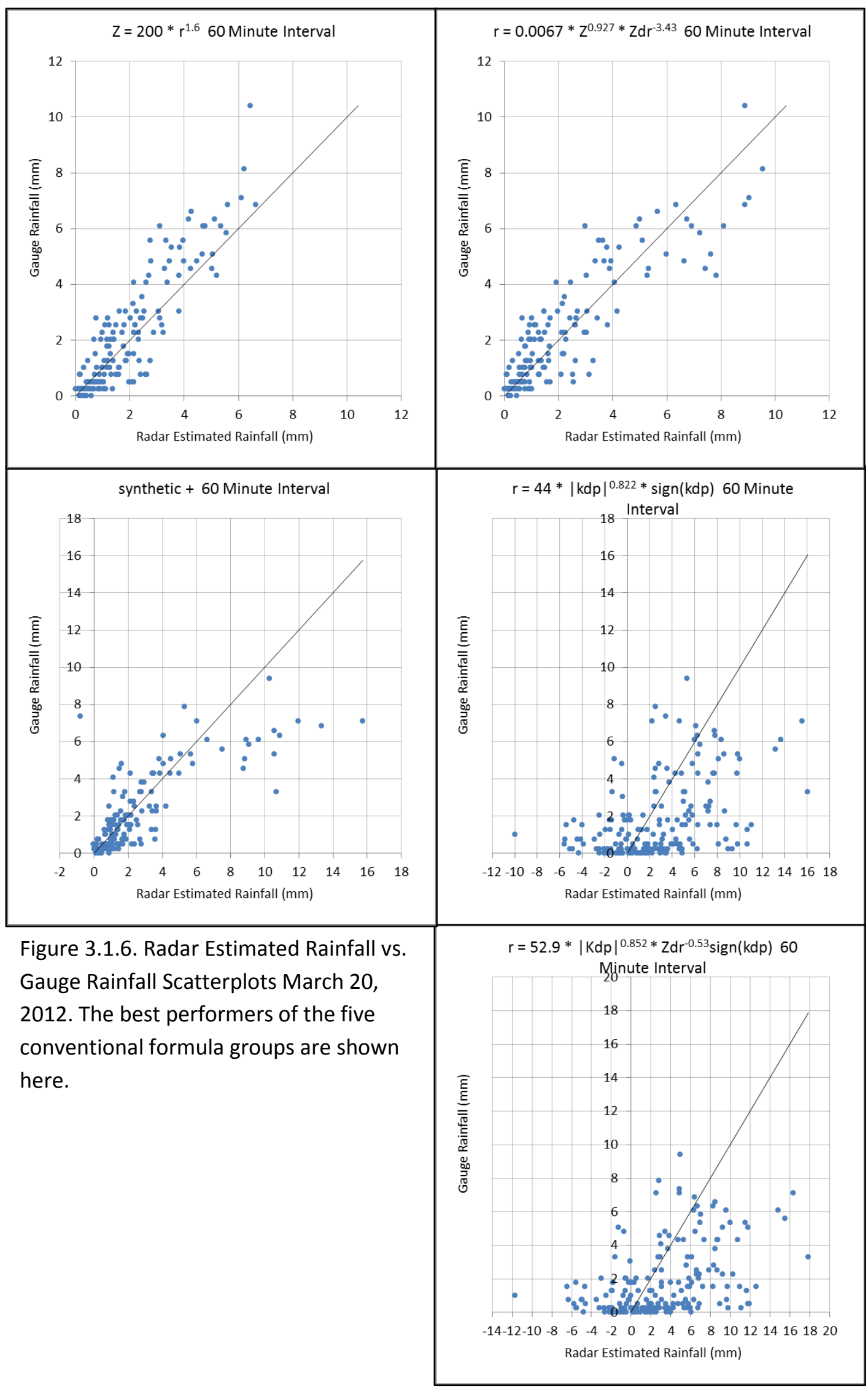



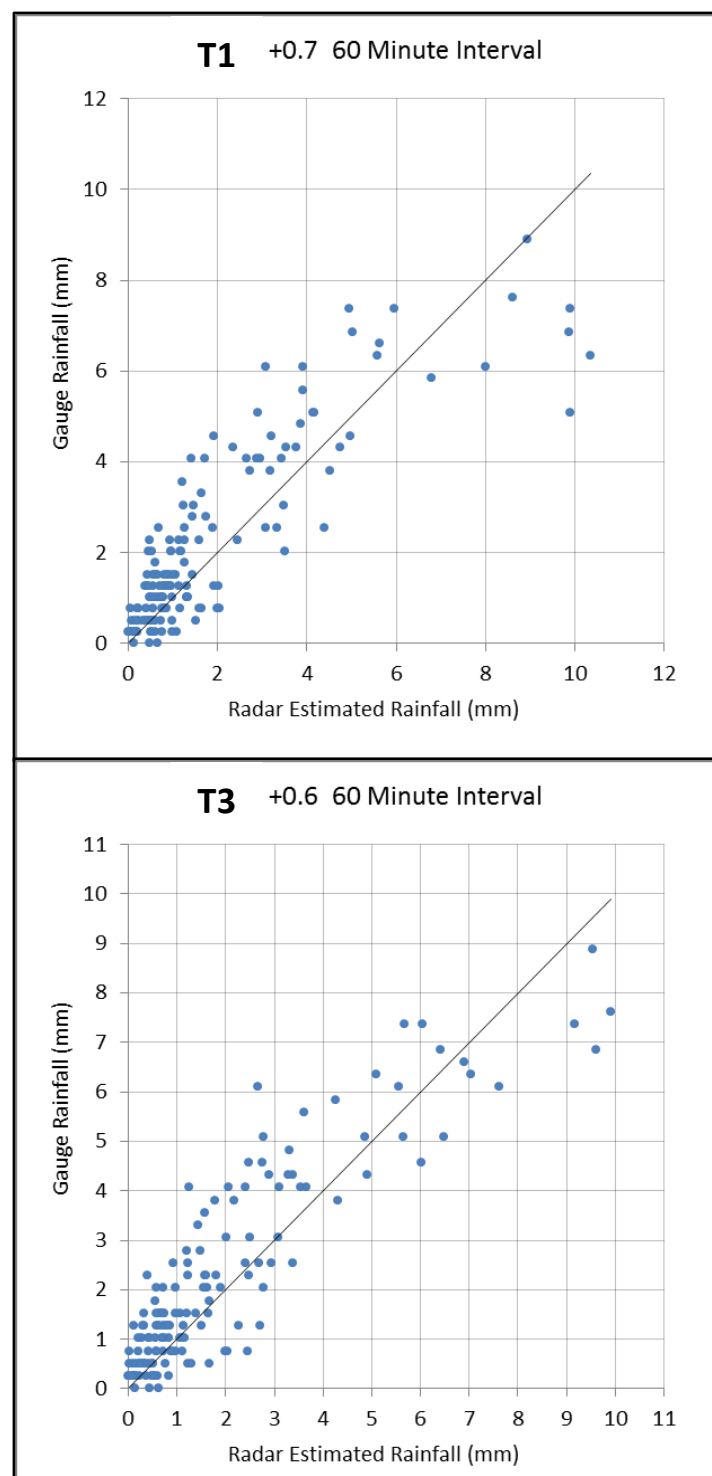

T5 +1.4 60 Minute Interval

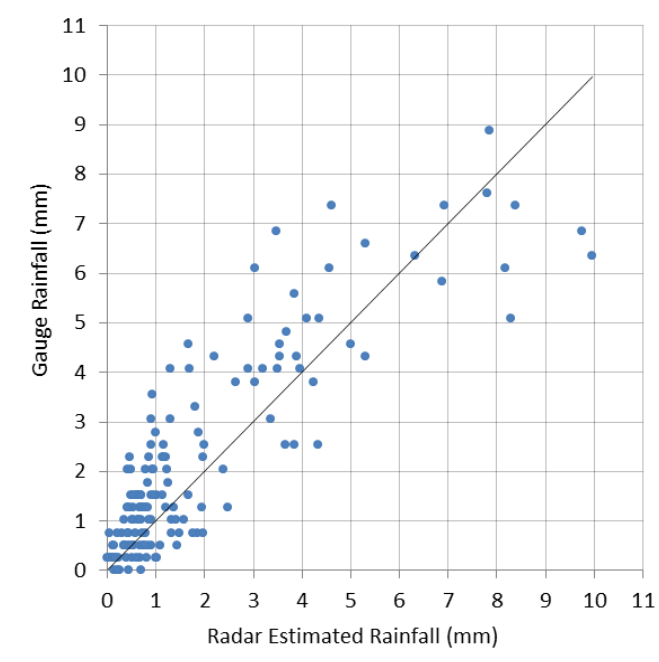

T2 +1.2 60 Minute Interval

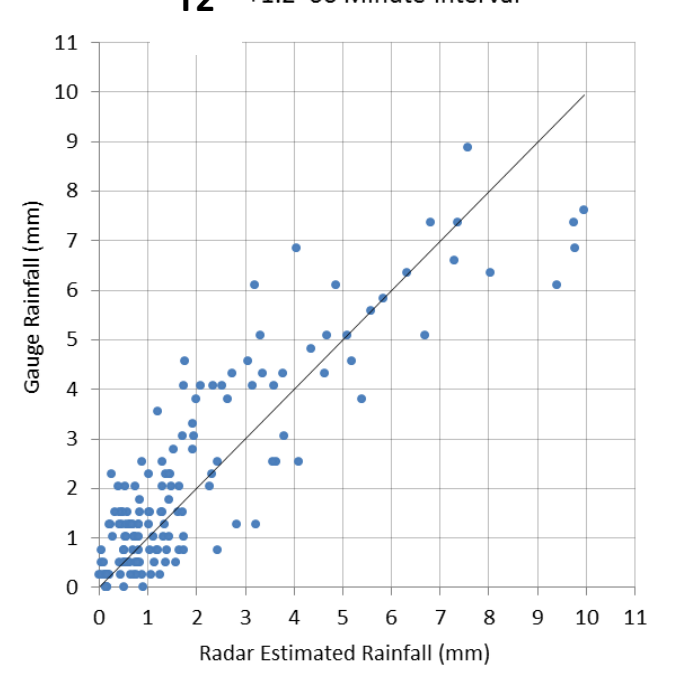

T4 +1.2 60 Minute Interval

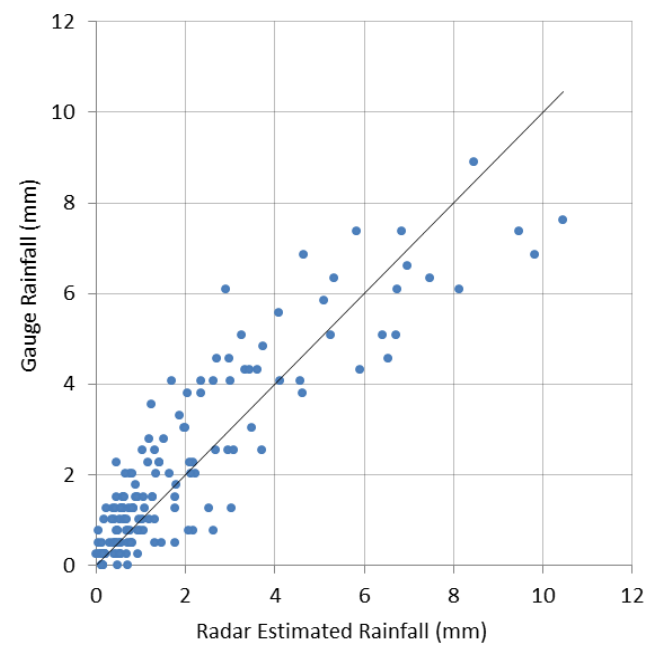

Figure 3.1.7. Radar Estimated Rainfall vs. Gauge Rainfall Scatterplots March 20, 2012. The best performers of the five trace techniques are shown here. 


\subsection{April 13, 2012}

The morning hours of April 13, 2012, saw precipitation overrun a warm front in the central United States. Figure 3.2.1 is an HPC surface analysis for 12 UTC of April 13, 2012. Figure 3.2.2 shows humidity versus height in percentile format for 04 UTC through 13 UTC of April 13. An exceptionally dry layer was present at about 500 meters above sea level throughout the examined period. Figure 3.2.3 is a hodograph for April 13. In the hodograph, a southeasterly wind at the surface transitions to southerly at 900 millibars, and southwesterly above 900.

\subsubsection{Conventional Formulas}

Due to extreme noise in the $K_{D P}$ data, formulas involving $K_{D P}$ were not evaluated for the precipitation events of April 13, April 14, and April 15 of 2012.

Table 3.2.1 shows the performance of the conventional techniques for this event. Of the conventional formulas evaluated, (5), was most effective. Three outliers are present in formulas (3), (12), and (13). (3) is the formula associated with tropical rain, so it is not unexpected that it would perform poorly in a frontal situation like this. (12) and (13) maybe have been affected by the $Z_{D R}$ bias mentioned in Chapter 2. A general positive bias was present for the conventional formulas tested in this event. Overall the conventional formulas performed worse on the FAD and FRMSE parameters when compared to March 20 of 2012. 


\subsubsection{Tracing Techniques}

Table 3.2.2 shows the performance of the various trace techniques and their permutations for this event. Techniques T2, T4, and T5 were most effective at the highest range of $+Z_{D R}$ values (2.6). Even then the associated values for FAD and FRMSE were higher for these than for techniques T1 and T3. The extremely dry layer at 500 meters is likely the cause of this. The techniques that take into account evaporation were much better suited to handle the precipitation reaching the ground.

The T4 technique performed the best out of the techniques that did not take into account evaporation. It is worth noting that the change from $+2.4 Z_{D R}$ to $+2.6 Z_{D R}$ resulted in improvements for the FAD and FRMSE values for the T2, T4, and T5 techniques. However, at this point the biases switched sign, so any significant further improvements from additional increases in $Z_{D R}$ were unlikely. $T 1$ values for $F A D$ and FRMSE were smaller than those for T3 but not by a great margin.

A significant decrease in FAD and FRMSE occurred with T1 and T3 at their best ZDR adjustment compared to the conventional techniques for this event. 


\subsubsection{Graphical Evaluation}

Figure 3.2.4 is a histogram showing the FAD performance of each evaluated technique for this event. Figure 3.2.5 is the equivalent histogram showing FRMSE performance.

Figure 3.2.6 shows a combination of scatterplots of radar-derived rainfall versus rain gauge rainfall for the conventional formulas. The techniques illustrated were the top performers in each category. Figure 3.2.7 is identical to 3.2.6, except it depicts the best performers of the trace techniques. 


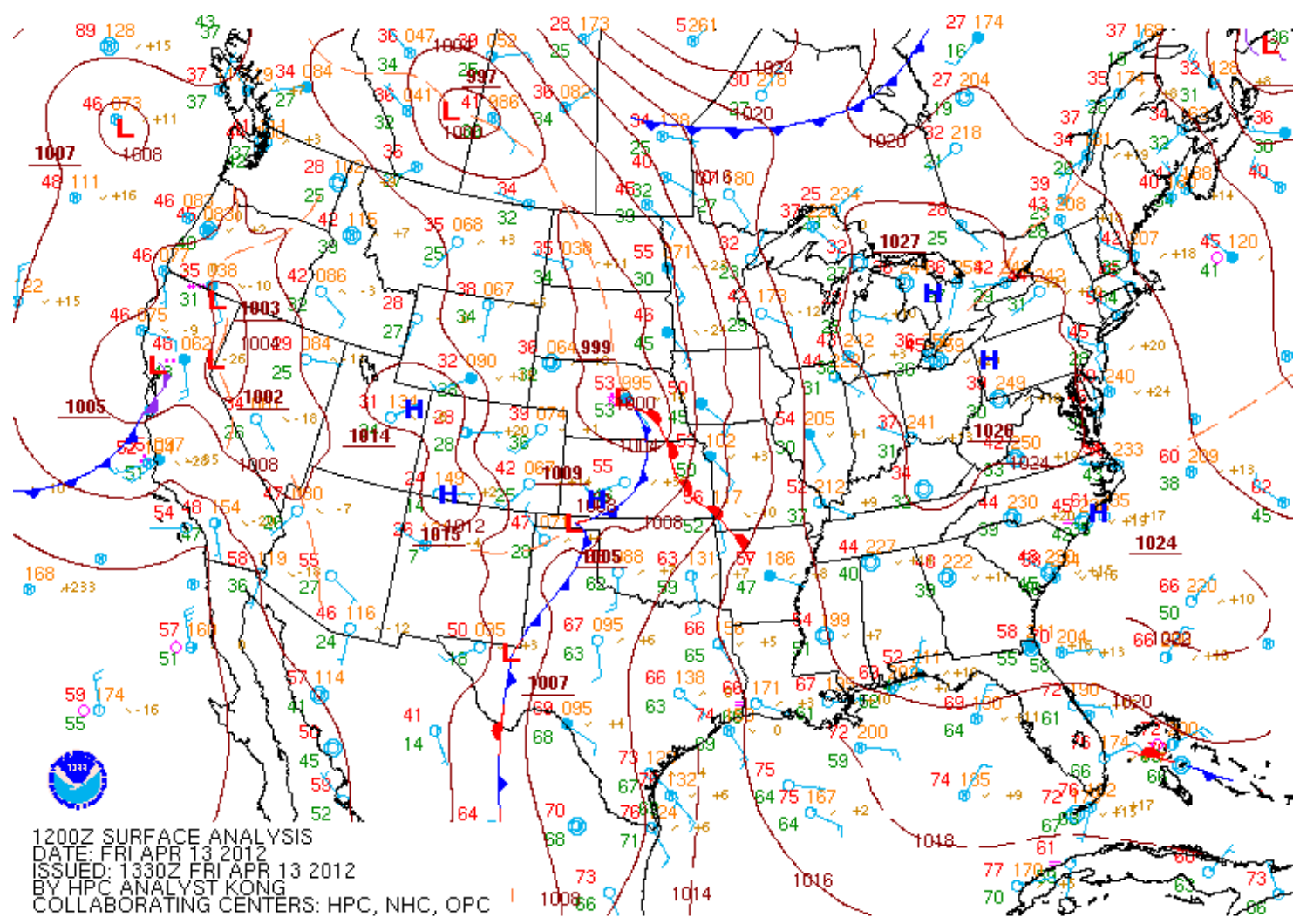

Figure 3.2.1. Surface Analysis 1200 UTC April 13, 2012.

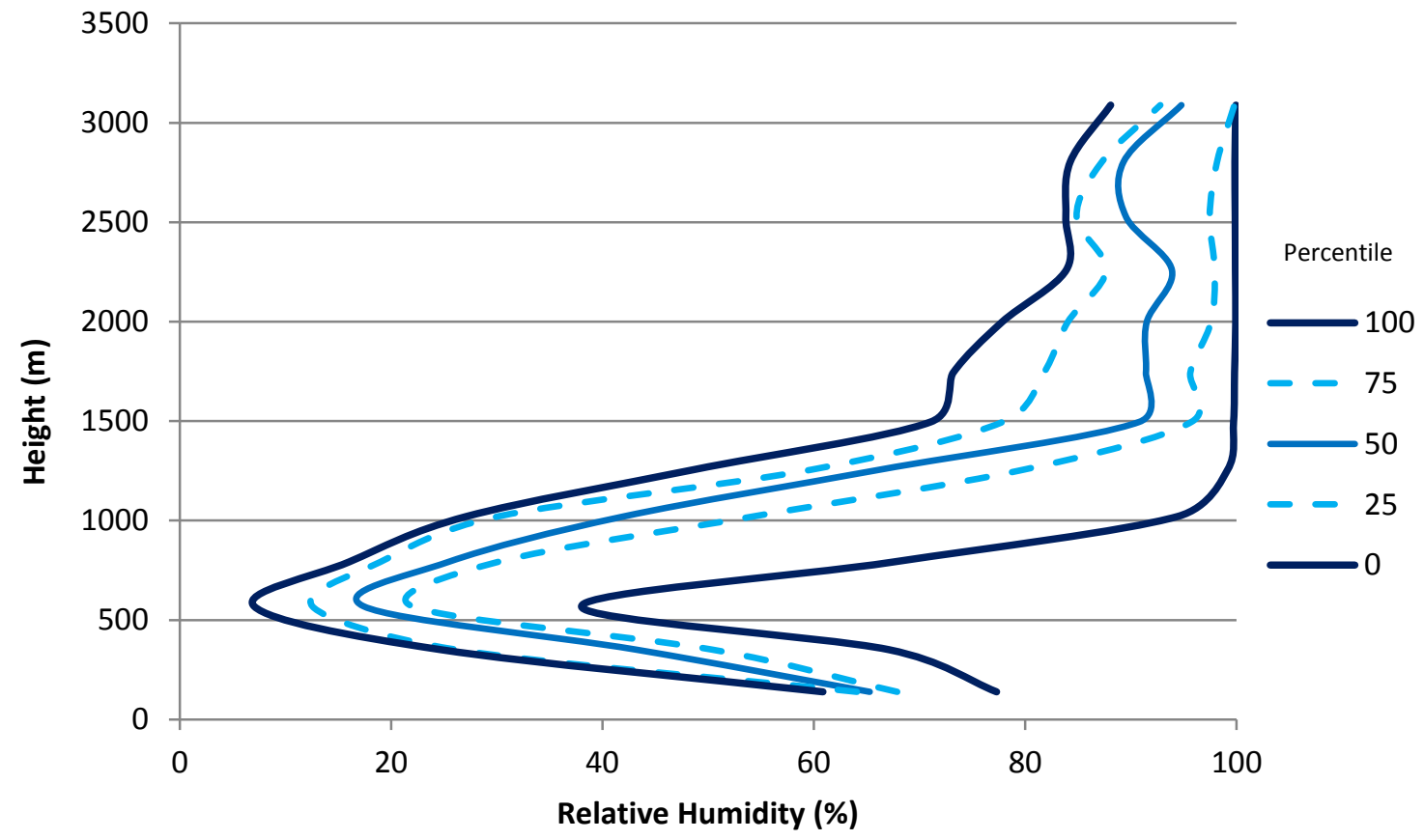

Figure 3.2.2. Relative Humidity Profile April 13, 2012. 


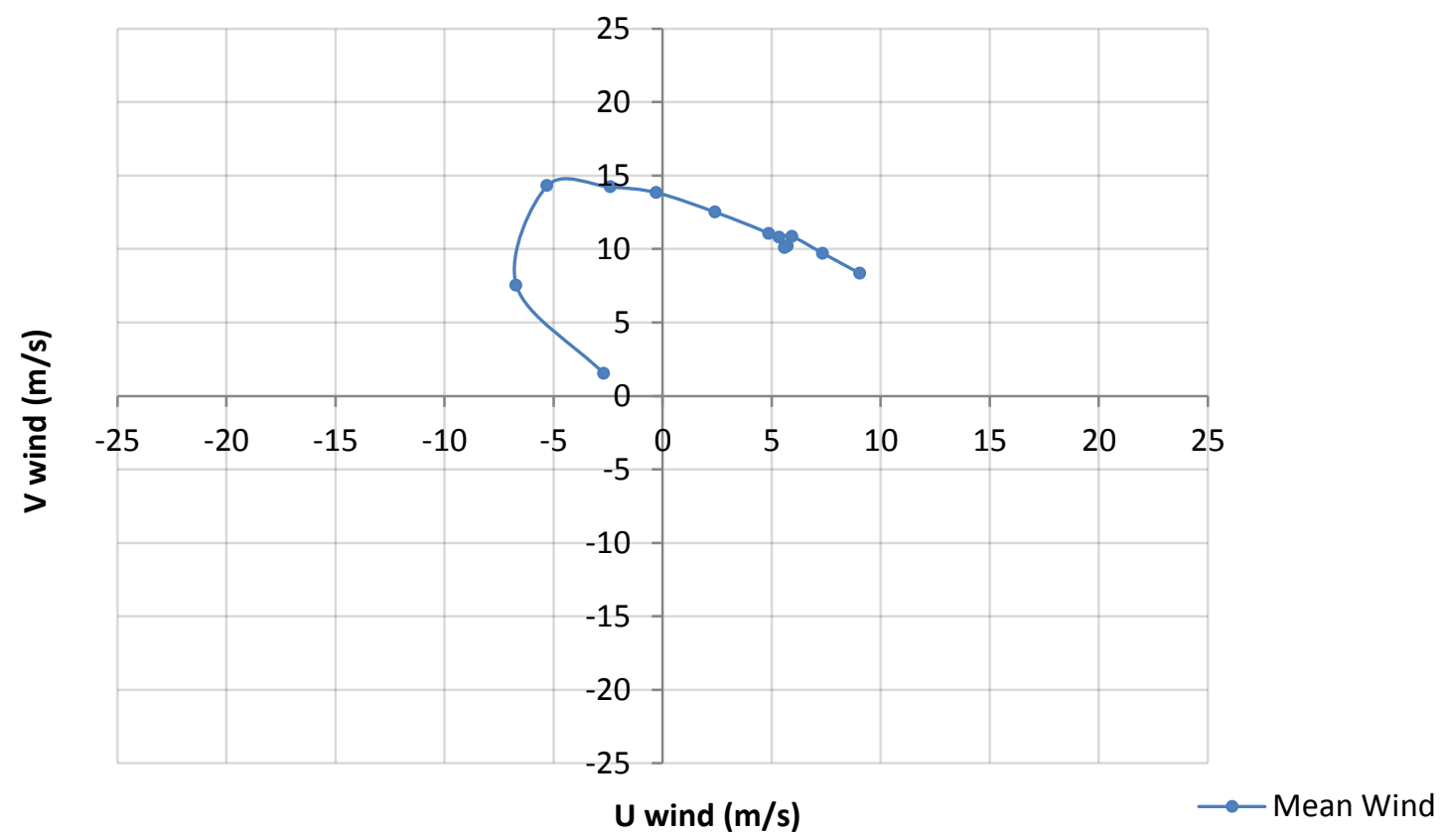

Figure 3.2.4. Hodograph April 13, 2012. 
Table 3.2.1 Conventional Techniques, April 13, 2012

\begin{tabular}{lccccc}
\hline$Z=a R^{b}$ & $\#$ & $\mathrm{FB}(\%)$ & $\mathrm{FAD}(\%)$ & $\mathrm{FRMSE}(\%)$ & $\mathrm{FSD}(\%)$ \\
\hline 1 & 24 & 45.79 & 63.72 & 84.15 & 70.60 \\
2 & 22 & 50.36 & 63.52 & 81.19 & 63.68 \\
3 & 22 & 164.17 & 164.17 & 217.20 & 142.21 \\
4 & 22 & 39.83 & 58.05 & 73.85 & 62.18 \\
5 & 20 & -1.28 & 47.50 & 58.89 & 58.88 \\
\hline$R\left(Z, Z_{D R}\right)=a Z^{b} Z_{d r}{ }^{c}$ & & & & & \\
\hline 12 & 22 & 55.18 & 75.15 & 106.58 & 91.18 \\
13 & 24 & 60.32 & 86.69 & 132.68 & 118.17 \\
14 & 22 & 23.85 & 58.35 & 77.07 & 73.29 \\
15 & 22 & 22.46 & 54.66 & 72.10 & 68.52 \\
16 & 22 & 20.82 & 57.35 & 75.12 & 72.17 \\
\hline
\end{tabular}


Table 3.2.2 Trace Techniques, April 13, 2012

\begin{tabular}{|c|c|c|c|c|c|c|}
\hline & $Z_{D R}+$ & $\#$ & $\mathrm{FB}(\%)$ & $\mathrm{FAD}(\%)$ & FRMSE(\%) & $\mathrm{FSD}(\%)$ \\
\hline \multirow[t]{14}{*}{ T1 } & 0 & 19 & -10.07 & 20.41 & 26.55 & 24.57 \\
\hline & 0.2 & 19 & -7.57 & 19.48 & 26.04 & 24.92 \\
\hline & 0.3 & 19 & -6.70 & 19.28 & 26.08 & 25.20 \\
\hline & 0.4 & 19 & -6.01 & 18.99 & 26.24 & 25.55 \\
\hline & 0.5 & 19 & -5.61 & 18.89 & 26.66 & 26.07 \\
\hline & 0.6 & 19 & -5.45 & 19.64 & 27.47 & 26.92 \\
\hline & 0.7 & 19 & -5.55 & 20.91 & 28.64 & 28.10 \\
\hline & 0.8 & 20 & -5.59 & 22.52 & 31.05 & 30.54 \\
\hline & 0.9 & 20 & -6.18 & 23.99 & 33.26 & 32.68 \\
\hline & 1 & 20 & -7.00 & 25.76 & 35.70 & 35.01 \\
\hline & 1.1 & 20 & -8.65 & 26.75 & 36.99 & 35.96 \\
\hline & 1.5 & 19 & -17.15 & 29.92 & 40.57 & 36.77 \\
\hline & 2 & 19 & -29.37 & 36.32 & 49.31 & 39.60 \\
\hline & 2.6 & 19 & -42.67 & 46.60 & 62.30 & 45.39 \\
\hline \multirow[t]{12}{*}{$\mathrm{T} 2$} & 0.4 & 27 & 183.59 & 183.59 & 243.33 & 159.70 \\
\hline & 0.6 & 27 & 148.50 & 148.50 & 200.95 & 135.37 \\
\hline & 0.8 & 26 & 120.03 & 120.03 & 165.76 & 114.31 \\
\hline & 1 & 25 & 96.73 & 98.64 & 138.47 & 99.09 \\
\hline & 1.2 & 25 & 77.98 & 82.13 & 120.32 & 91.63 \\
\hline & 1.4 & 25 & 61.55 & 69.04 & 102.93 & 82.49 \\
\hline & 1.6 & 25 & 46.95 & 58.15 & 88.13 & 74.59 \\
\hline & 1.8 & 25 & 33.58 & 51.28 & 75.78 & 67.94 \\
\hline & 2 & 23 & 21.52 & 46.14 & 64.53 & 60.84 \\
\hline & 2.2 & 23 & 10.97 & 41.97 & 58.85 & 57.82 \\
\hline & 2.4 & 23 & 1.64 & 39.71 & 56.11 & 56.08 \\
\hline & 2.6 & 23 & -7.08 & 39.36 & 55.49 & 55.03 \\
\hline \multirow[t]{14}{*}{ T3 } & 0 & 19 & -5.64 & 21.69 & 28.56 & 28.00 \\
\hline & 0.2 & 19 & -5.37 & 22.15 & 29.87 & 29.38 \\
\hline & 0.3 & 20 & -5.26 & 22.44 & 31.34 & 30.90 \\
\hline & 0.4 & 21 & -5.56 & 23.09 & 33.18 & 32.71 \\
\hline & 0.5 & 21 & -6.35 & 24.08 & 34.41 & 33.82 \\
\hline & 0.6 & 21 & -7.39 & 25.07 & 35.78 & 35.01 \\
\hline & 0.7 & 22 & -8.35 & 26.25 & 38.12 & 37.19 \\
\hline & 0.8 & 22 & -9.80 & 26.94 & 39.43 & 38.19 \\
\hline & 0.9 & 22 & -11.45 & 27.53 & 40.76 & 39.12 \\
\hline & 1 & 22 & -13.10 & 28.58 & 42.23 & 40.15 \\
\hline & 1.1 & 22 & -14.77 & 29.73 & 43.78 & 41.21 \\
\hline & 1.5 & 22 & -21.50 & 34.25 & 50.66 & 45.87 \\
\hline & 2 & 20 & -32.46 & 39.42 & 57.16 & 47.05 \\
\hline & 2.6 & 19 & -44.93 & 49.09 & 66.86 & 49.51 \\
\hline
\end{tabular}


Table 3.2.2 Trace Techniques, April 13, 2012 (Continued)

\begin{tabular}{ccccccc}
\hline & $Z_{D R}+$ & $\#$ & $\mathrm{FB}(\%)$ & $\mathrm{FAD}(\%)$ & $\mathrm{FRMSE}(\%)$ & $\mathrm{FSD}(\%)$ \\
\hline $\mathrm{T} 4$ & 0.4 & 22 & 179.80 & 179.80 & 222.23 & 130.61 \\
& 0.6 & 22 & 145.17 & 145.17 & 183.62 & 112.44 \\
0.8 & 22 & 117.32 & 117.32 & 154.41 & 100.40 \\
1 & 22 & 94.54 & 98.09 & 131.62 & 91.57 \\
& 22 & 76.01 & 83.31 & 114.42 & 85.53 \\
& 22 & 59.78 & 70.27 & 98.04 & 77.71 \\
& 1.4 & 45.34 & 58.84 & 84.21 & 70.96 \\
1.6 & 22 & 32.11 & 51.47 & 72.71 & 65.23 \\
1.8 & 22 & 20.61 & 46.47 & 64.81 & 61.44 \\
& 22 & 10.14 & 41.97 & 59.28 & 58.40 \\
& 22 & 0.87 & 39.27 & 56.56 & 56.55 \\
& 22 & -7.79 & 38.46 & 55.86 & 55.32 \\
\hline 2.4 & 22 & 188.04 & 188.04 & 231.25 & 134.61 \\
& 2.6 & 153.76 & 153.76 & 193.41 & 117.32 \\
& 22 & 126.82 & 126.82 & 162.63 & 101.81 \\
& 0.4 & 25 & 105.51 & 105.51 & 142.80 & 96.23 \\
0.6 & 25 & 85.62 & 85.76 & 120.03 & 84.11 \\
0.8 & 24 & 68.40 & 71.40 & 102.38 & 76.18 \\
1 & 24 & 52.70 & 60.04 & 85.83 & 67.75 \\
1.2 & 23 & 39.10 & 51.85 & 72.66 & 61.25 \\
1.4 & 23 & 26.57 & 44.85 & 61.45 & 55.41 \\
1.6 & 23 & 15.07 & 40.50 & 52.29 & 50.07 \\
1.8 & 23 & 5.06 & 37.00 & 48.20 & 47.93 \\
2 & 23 & -4.35 & 35.20 & 45.27 & 45.06 \\
\hline 2.2 & 22 & & & &
\end{tabular}




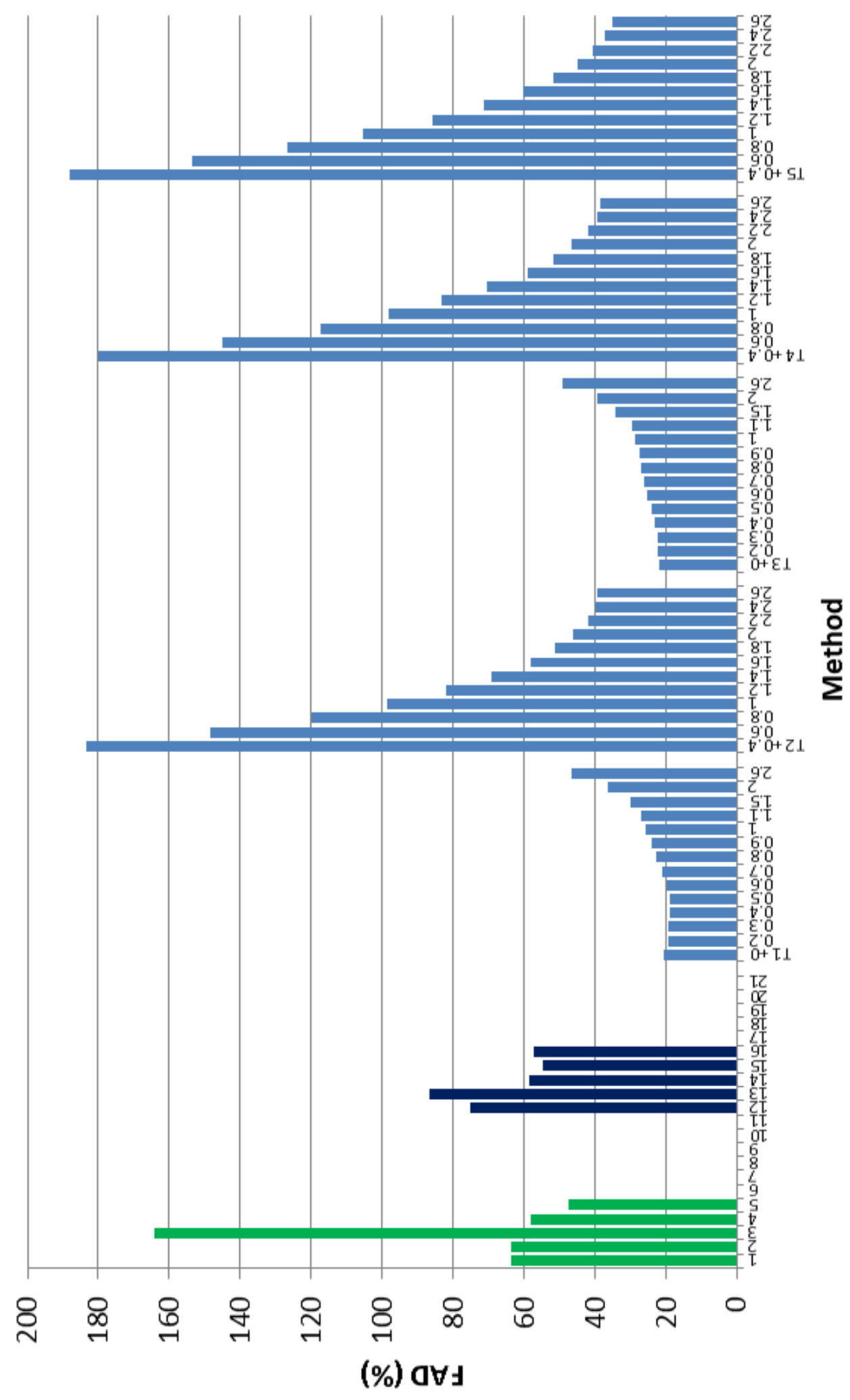

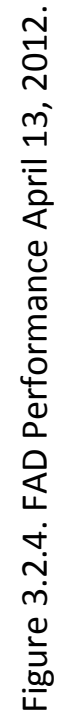




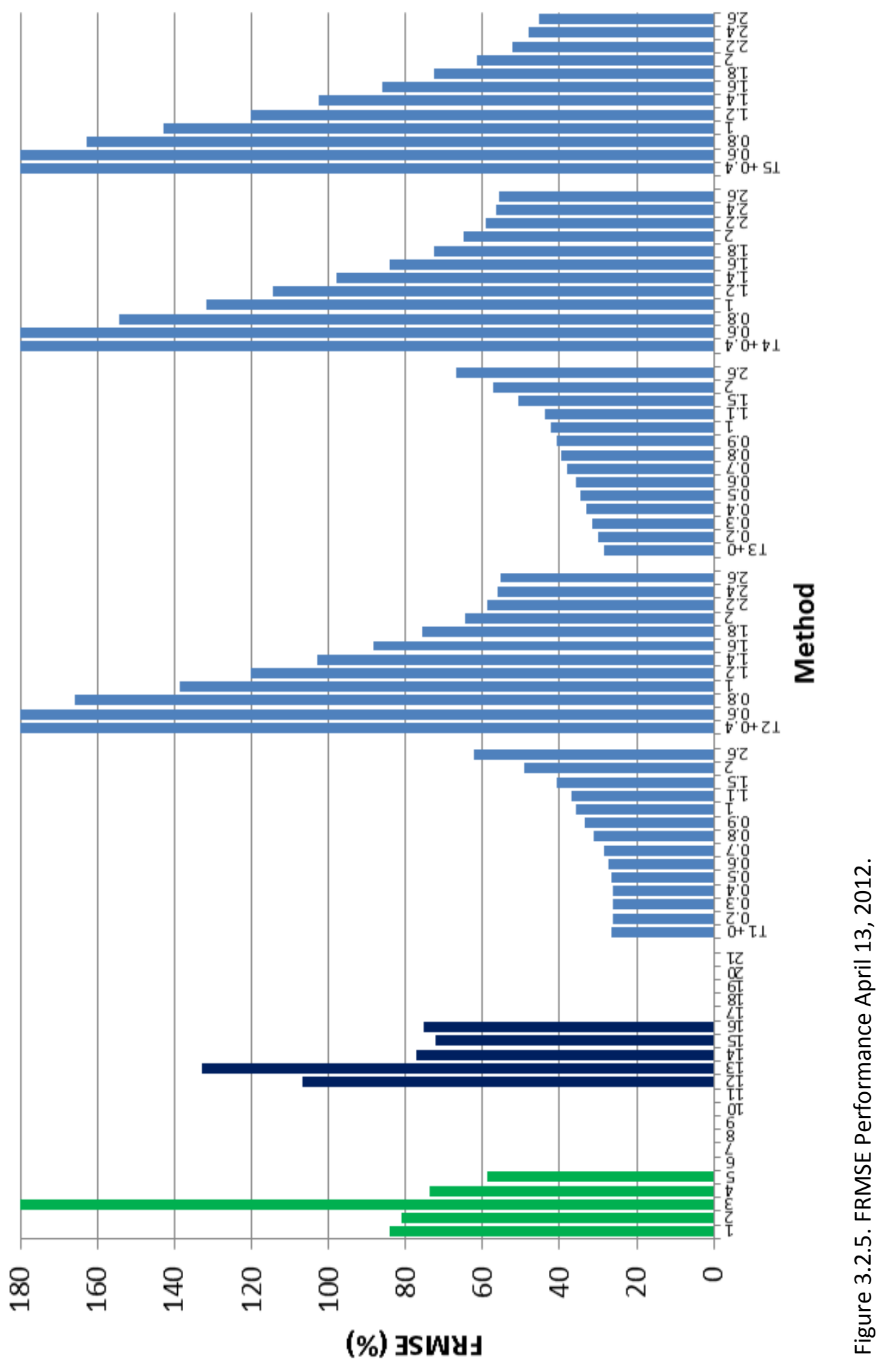




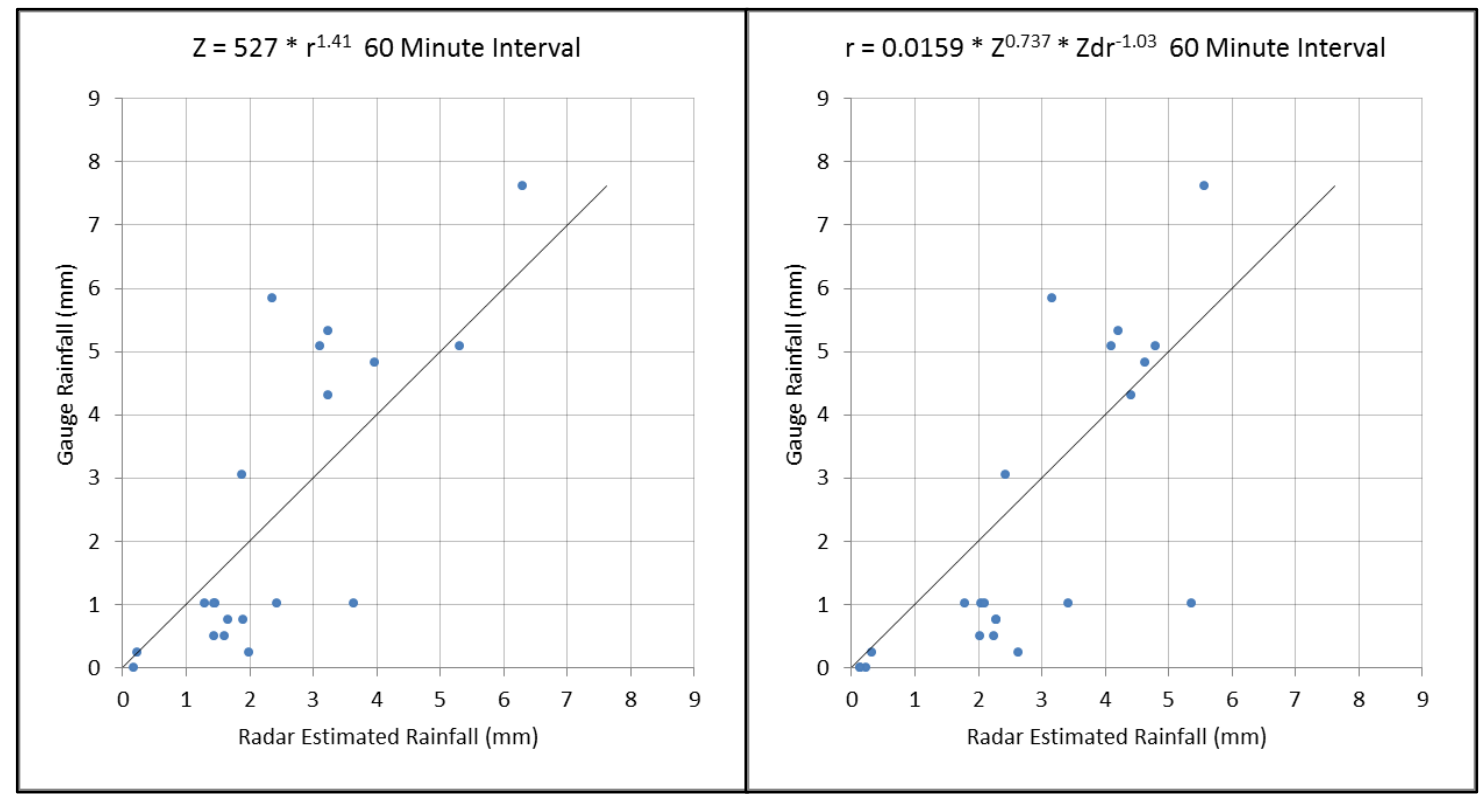

Figure 3.2.4. Radar Estimated Rainfall vs. Gauge Rainfall Scatterplots April 13, 2012. The best performers of the two evaluated conventional formula groups are shown here. 


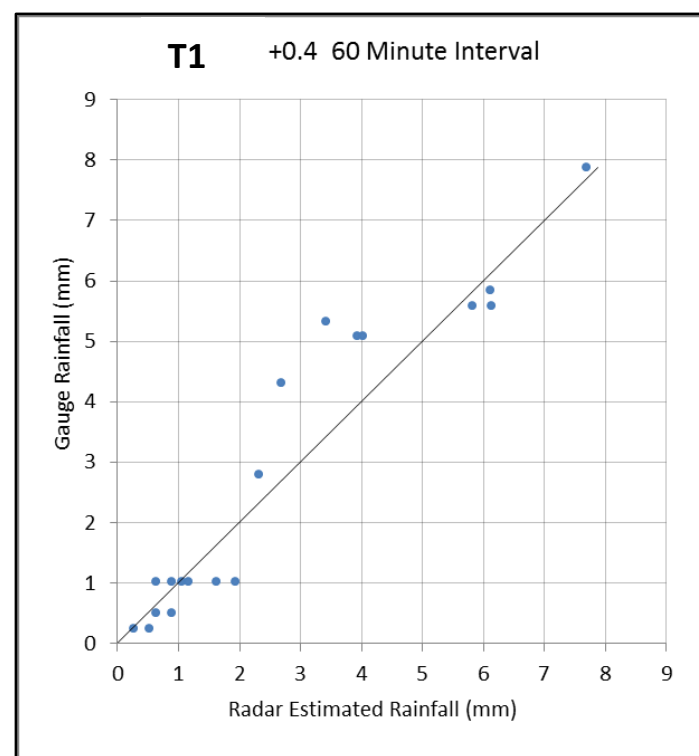

T3 +0 60 Minute Interval

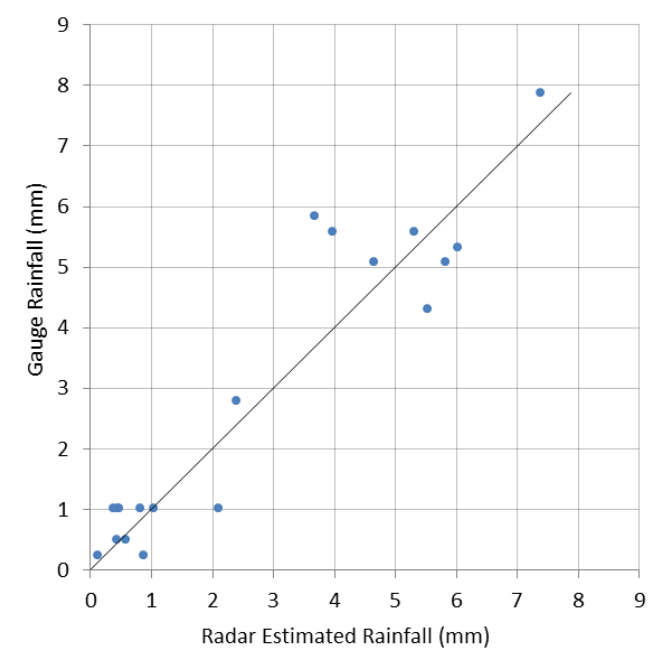

T5 +2.6 60 Minute Interval

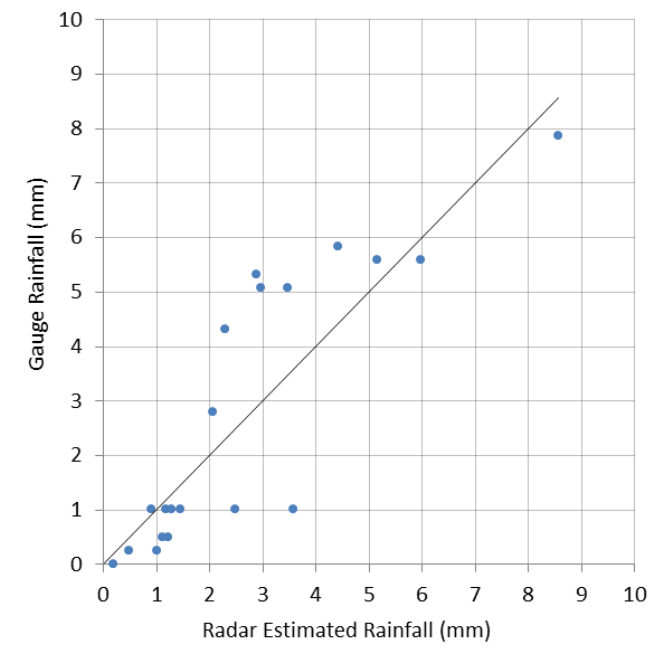

T2 +2.6 60 Minute Interval

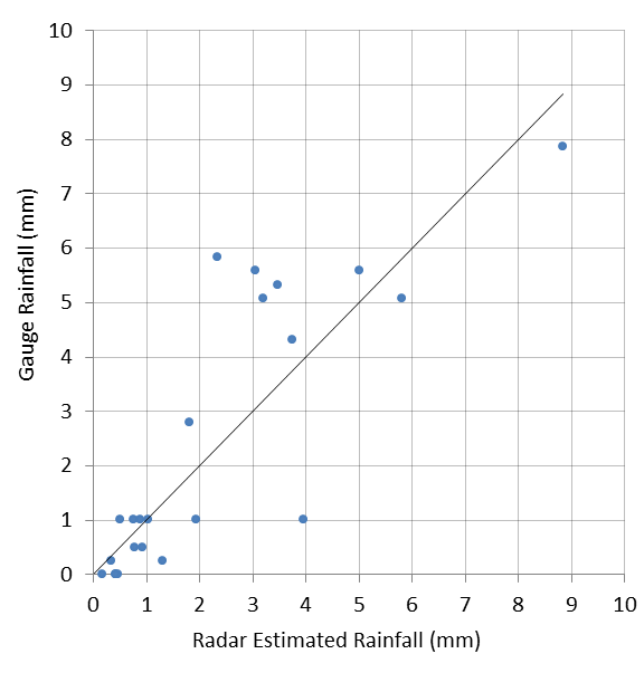

T4 +2.6 60 Minute Interval

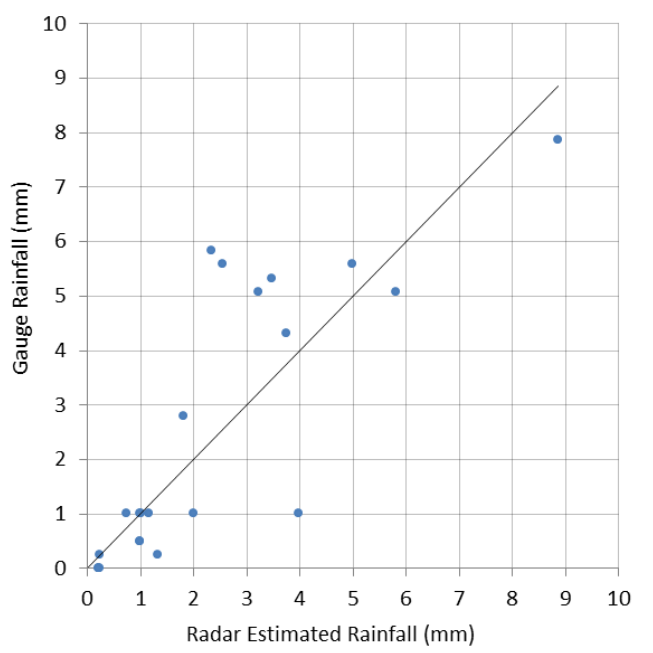

Figure 3.2.5. Radar Estimated Rainfall vs. Gauge Rainfall Scatterplots April 13, 2012. The best performers of the five trace techniques are shown here. 


\subsection{April 14, 2012}

The same system that brought precipitation to central Missouri on April 13 continued to affect the area on the $14^{\text {th }}$. Figure 3.3.1 is an HPC surface analysis for 09 UTC of April 14, 2012. Figure 3.3.2 shows humidity versus height in percentile format for 01 UTC through 10 UTC of April 14. The dry layer at 500 meters was still present, but was much weaker in magnitude. Figure 3.3.3 is a hodograph for the $14^{\text {th }}$. The hodograph indicates the wind direction on the $14^{\text {th }}$ became more westerly (or less easterly) at all levels shown. Figure 3.3.4 is a four panel radar image from KLSX for 0924 UTC of April 14.

\subsubsection{Conventional Formulas}

Due to extreme noise in the $K_{D P}$ data, formulas involving $K_{D P}$ were not evaluated for the precipitation events of April 13, April 14, and April 15 of 2012.

Table 3.3.1 shows the performance of the conventional techniques for this event. (2), the standard WSR-88D formula, was most effective of all the conventional formulas. Only one outlier, (3), was present, likely for the same reasons as it was an outlier on the $13^{\text {th }}$. There was a mix of positive and negative biases for the conventional formulas on this date.

\subsubsection{Tracing Techniques}

Table 3.3.2 shows the performance of the various trace techniques and their permutations for this event. Not many patterns were present for the tracing technique results on the $14^{\text {th }}$ of April, 2012. The optimal ZDR adjustment varied considerably 
across the tracing techniques. Several anomalously low FAD were present, suggesting outlying data points.

\subsubsection{Graphical Evaluation}

Figure 3.3.5 is a histogram showing the FAD performance of each evaluated technique for this event. Figure 3.3.6 is the equivalent histogram showing FRMSE performance.

Figure 3.3.7 shows a combination of scatterplots of radar-derived rainfall versus rain gauge rainfall for the conventional formulas. The techniques illustrated were the top performers in each category. Figure 3.3.8 is identical to 3.3.7, except it depicts the best performers of the trace techniques. 


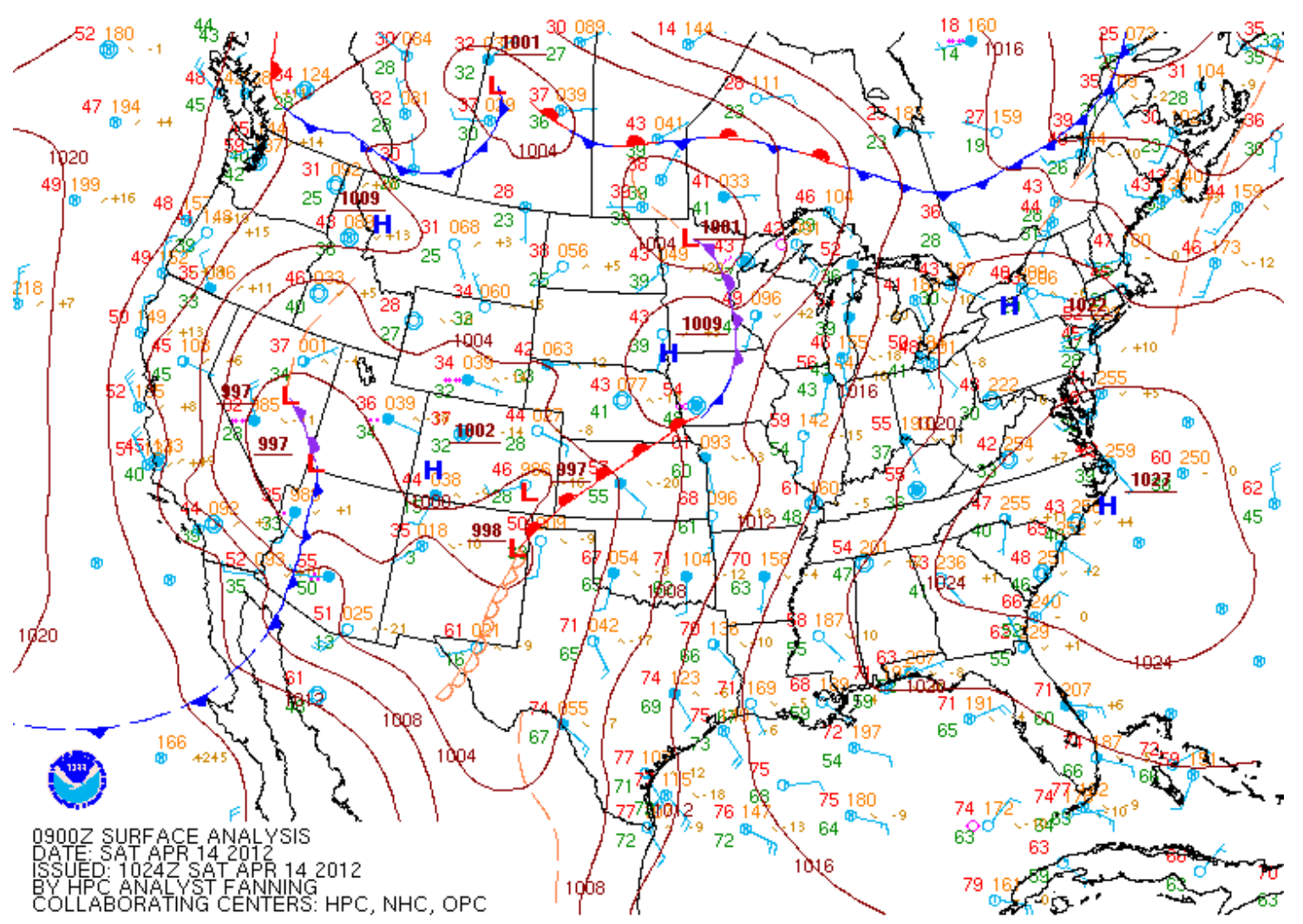

Figure 3.3.1. Surface Analysis 0900 UTC April 14, 2012.

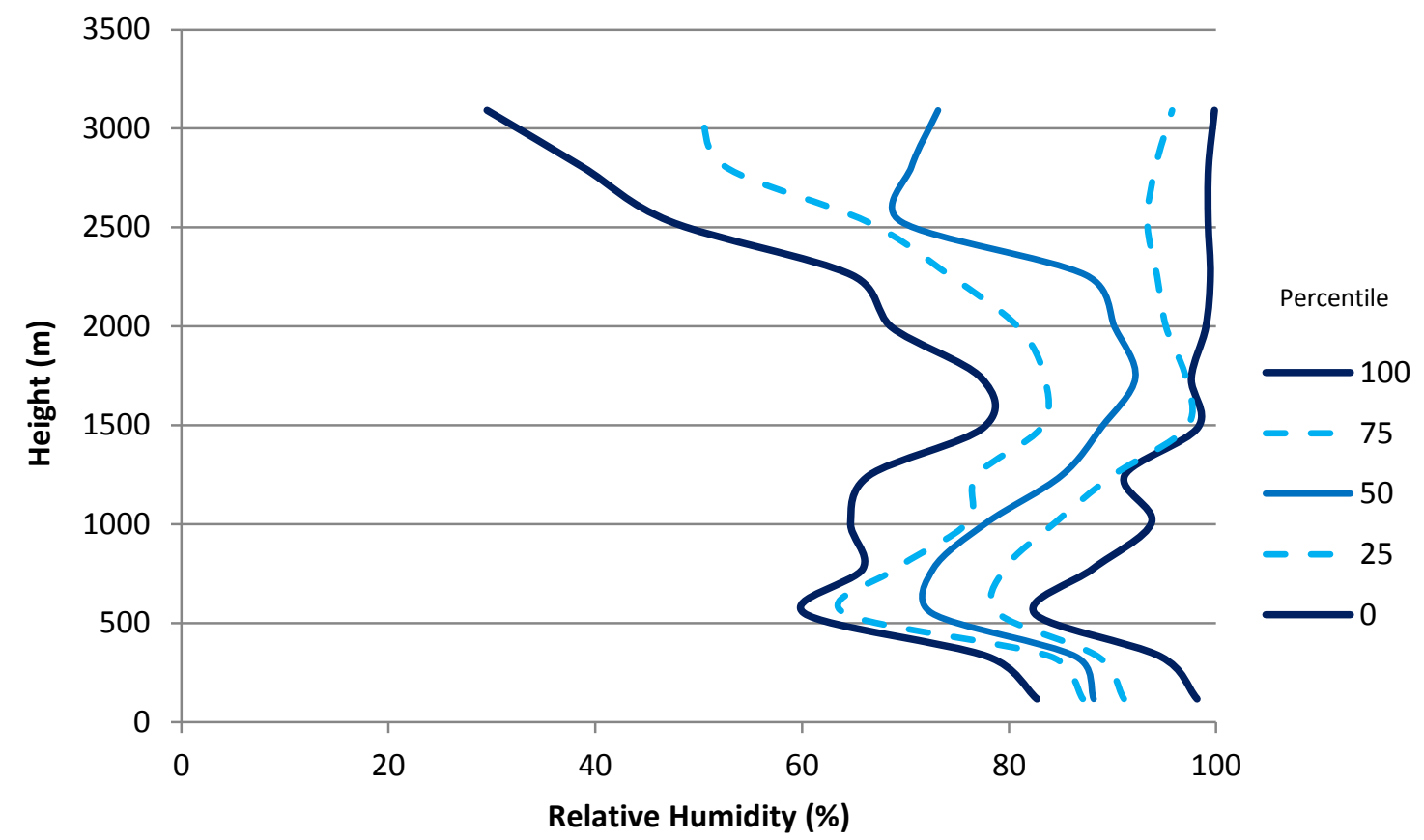

Figure 3.3.2. Relative Humidity Profile April 14, 2012. 


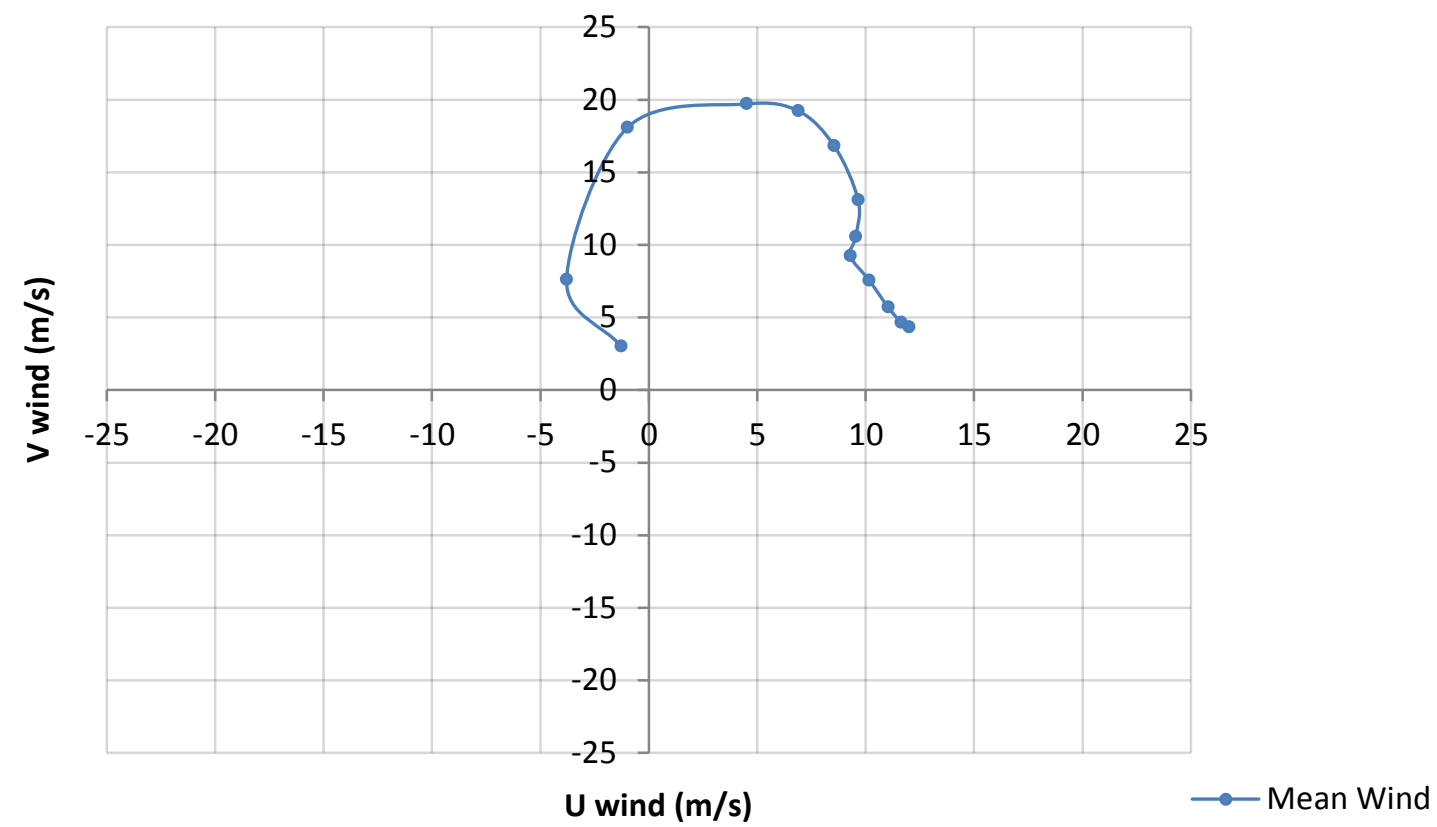

Figure 3.3.3. Hodograph April 14, 2012. 


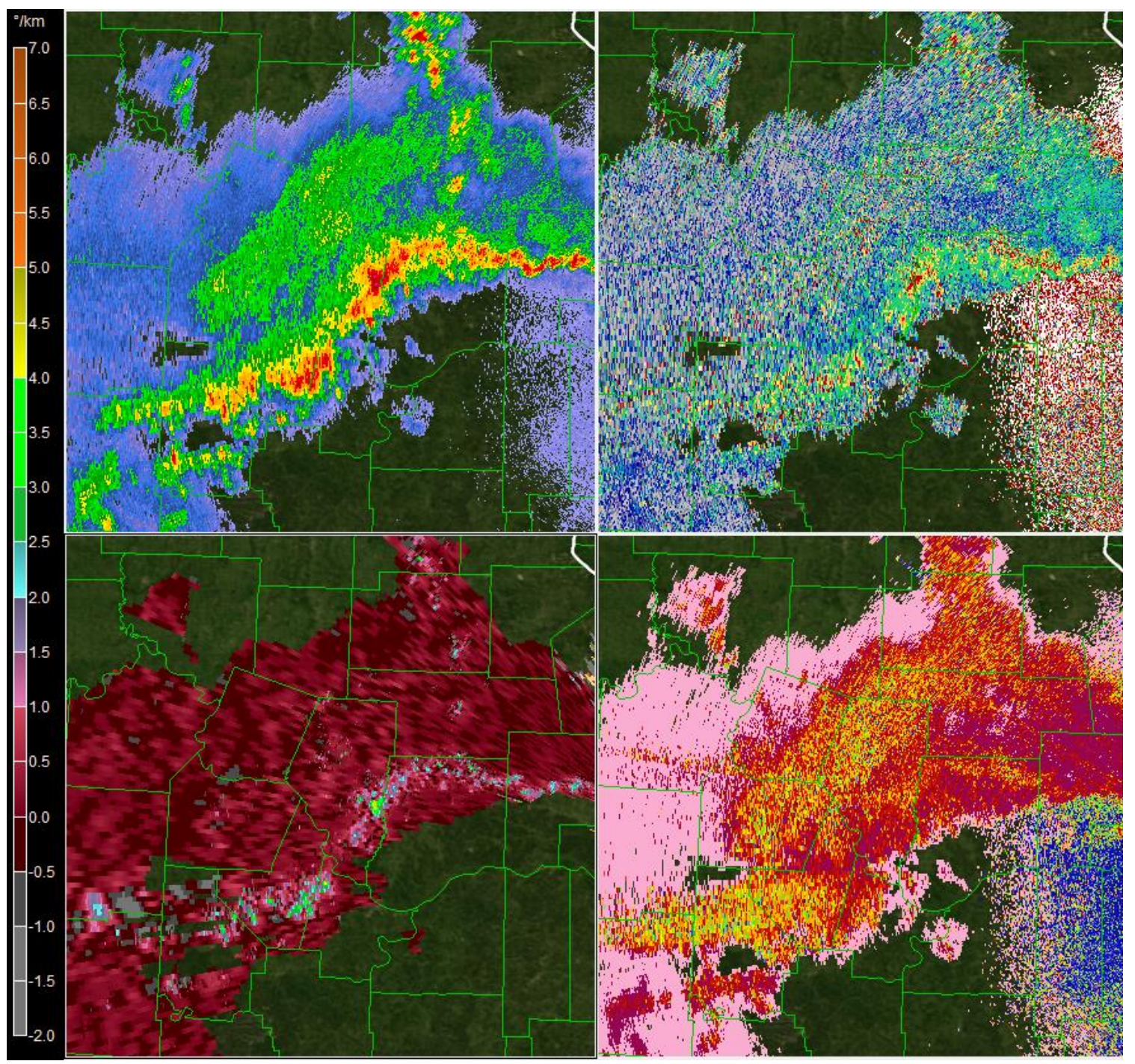

Figure 3.3.4. Four Panel Radar Image for 0924 UTC April 14, 2012. The top left is reflectivity $(Z)$, top right is differential reflectivity $\left(Z_{D R}\right)$, bottom left is specific phase differential $\left(K_{D P}\right)$, and bottom right is correlation coefficient. The scale on the left corresponds to the bottom left image $\left(K_{D P}\right)$. The elevation angle used here was $0.5^{\circ}$. 
Table 3.3.1 Conventional Techniques, April 14, 2012

\begin{tabular}{lccccc}
\hline$Z=a R^{b}$ & $\#$ & $\mathrm{FB}(\%)$ & $\mathrm{FAD}(\%)$ & $\mathrm{FRMSE}(\%)$ & $\mathrm{FSD}(\%)$ \\
\hline 1 & 29 & -10.95 & 41.62 & 68.85 & 67.97 \\
2 & 28 & -3.97 & 36.03 & 62.18 & 62.05 \\
3 & 29 & 79.68 & 82.23 & 156.05 & 134.17 \\
4 & 28 & -11.58 & 39.31 & 66.10 & 65.08 \\
5 & 24 & -37.45 & 49.69 & 83.86 & 75.03 \\
\hline$R\left(Z, Z_{D R}\right)=a Z^{b} Z_{d r}^{c}$ & & & & \\
\hline 12 & 26 & -3.77 & 33.85 & 66.38 & 66.27 \\
13 & 26 & -5.16 & 36.62 & 69.18 & 68.99 \\
14 & 26 & -25.02 & 46.29 & 76.40 & 72.19 \\
15 & 28 & -25.50 & 46.81 & 79.85 & 75.66 \\
16 & 26 & -26.87 & 47.22 & 78.14 & 73.37 \\
\hline
\end{tabular}


Table 3.3.2 Trace Techniques, April 14, 2012

\begin{tabular}{|c|c|c|c|c|c|c|}
\hline & $Z_{D R}+$ & $\#$ & $\mathrm{FB}(\%)$ & FAD(\%) & FRMSE(\%) & $\mathrm{FSD}(\%)$ \\
\hline \multirow[t]{14}{*}{ T1 } & 0 & 31 & 56.17 & 64.08 & 126.38 & 113.21 \\
\hline & 0.2 & 28 & 48.06 & 56.55 & 108.84 & 97.65 \\
\hline & 0.3 & 28 & 44.39 & 53.14 & 103.57 & 93.58 \\
\hline & 0.4 & 28 & 40.89 & 50.12 & 99.08 & 90.25 \\
\hline & 0.5 & 27 & 37.04 & 46.70 & 92.74 & 85.02 \\
\hline & 0.6 & 25 & 33.06 & 43.06 & 85.03 & 78.34 \\
\hline & 0.7 & 25 & 29.43 & 39.67 & 81.18 & 75.66 \\
\hline & 0.8 & 25 & 25.74 & 36.41 & 77.93 & 73.55 \\
\hline & 0.9 & 25 & 22.07 & 33.69 & 74.76 & 71.43 \\
\hline & 1 & 25 & 18.51 & 31.43 & 72.14 & 69.73 \\
\hline & 1.1 & 25 & 14.92 & 29.21 & 69.93 & 68.32 \\
\hline & 1.5 & 25 & 0.65 & 30.57 & 65.81 & 65.81 \\
\hline & 2 & 24 & -15.99 & 35.51 & 67.17 & 65.24 \\
\hline & 2.6 & 23 & -34.30 & 45.74 & 79.10 & 71.27 \\
\hline \multirow[t]{12}{*}{$\mathrm{T} 2$} & 0.4 & 26 & 101.06 & 103.62 & 186.65 & 156.92 \\
\hline & 0.6 & 25 & 77.65 & 80.97 & 145.45 & 122.99 \\
\hline & 0.8 & 25 & 59.51 & 63.56 & 117.34 & 101.13 \\
\hline & 1 & 25 & 43.42 & 48.05 & 94.19 & 83.58 \\
\hline & 1.2 & 24 & 29.59 & 36.36 & 75.15 & 69.08 \\
\hline & 1.4 & 24 & 18.26 & 31.27 & 63.73 & 61.06 \\
\hline & 1.6 & 24 & 7.88 & 26.32 & 56.61 & 56.06 \\
\hline & 1.8 & 23 & -1.38 & 24.44 & 52.87 & 52.85 \\
\hline & 2 & 23 & -10.06 & 27.56 & 53.94 & 52.99 \\
\hline & 2.2 & 23 & -17.94 & 32.82 & 57.74 & 54.89 \\
\hline & 2.4 & 23 & -24.99 & 37.54 & 63.20 & 58.05 \\
\hline & 2.6 & 23 & -31.30 & 41.89 & 69.36 & 61.89 \\
\hline \multirow[t]{14}{*}{ T3 } & 0 & 23 & 12.25 & 28.02 & 67.28 & 66.16 \\
\hline & 0.2 & 23 & 6.22 & 26.30 & 61.97 & 61.65 \\
\hline & 0.3 & 23 & 2.96 & 25.88 & 59.76 & 59.69 \\
\hline & 0.4 & 23 & -0.22 & 26.38 & 58.07 & 58.07 \\
\hline & 0.5 & 23 & -3.25 & 27.30 & 56.93 & 56.84 \\
\hline & 0.6 & 23 & -6.10 & 28.08 & 56.29 & 55.96 \\
\hline & 0.7 & 23 & -8.87 & 28.76 & 56.16 & 55.46 \\
\hline & 0.8 & 23 & -11.51 & 29.73 & 56.52 & 55.33 \\
\hline & 0.9 & 23 & -14.68 & 31.55 & 57.50 & 55.59 \\
\hline & 1 & 23 & -17.64 & 33.25 & 58.86 & 56.15 \\
\hline & 1.1 & 23 & -20.47 & 34.89 & 60.52 & 56.95 \\
\hline & 1.5 & 23 & -30.62 & 40.96 & 68.97 & 61.81 \\
\hline & 2 & 23 & -42.47 & 49.49 & 82.23 & 70.41 \\
\hline & 2.6 & 23 & -54.46 & 58.62 & 97.98 & 81.45 \\
\hline
\end{tabular}


Table 3.3.2 Trace Techniques, April 14, 2012 (Continued)

\begin{tabular}{ccccccc}
\hline & $Z_{D R}+$ & $\#$ & $\mathrm{FB}(\%)$ & $\mathrm{FAD}(\%)$ & $\mathrm{FRMSE}(\%)$ & $\mathrm{FSD}(\%)$ \\
\hline $\mathrm{T} 4$ & 0.4 & 27 & 72.63 & 75.13 & 145.58 & 126.17 \\
& 0.6 & 26 & 53.29 & 56.54 & 112.71 & 99.32 \\
0.8 & 26 & 38.19 & 45.60 & 91.16 & 82.78 \\
& 26 & 24.44 & 36.27 & 74.28 & 70.14 \\
& 1.2 & 25 & 12.73 & 31.50 & 62.35 & 61.04 \\
1.4 & 25 & 3.19 & 28.45 & 57.44 & 57.35 \\
1.6 & 24 & -5.79 & 26.15 & 55.28 & 54.98 \\
& 23 & -13.75 & 28.95 & 56.23 & 54.52 \\
& 23 & -21.24 & 34.01 & 60.74 & 56.91 \\
& 23 & -28.04 & 38.64 & 66.52 & 60.32 \\
& 23 & -34.14 & 43.07 & 72.76 & 64.25 \\
& 23 & -39.62 & 47.21 & 79.03 & 68.38 \\
\hline T5 & 23 & 148.52 & 148.74 & 283.99 & 242.06 \\
& 23 & 121.55 & 121.83 & 228.09 & 193.01 \\
& 0.4 & 37 & 100.26 & 100.63 & 188.50 & 159.63 \\
0.6 & 34 & 82.33 & 82.92 & 154.28 & 130.47 \\
0.8 & 32 & 67.03 & 67.81 & 133.36 & 115.29 \\
1 & 29 & 53.44 & 57.19 & 116.11 & 103.08 \\
1.2 & 29 & 40.81 & 47.56 & 97.36 & 88.40 \\
1.4 & 29 & 29.41 & 38.96 & 83.74 & 78.41 \\
1.6 & 27 & 18.38 & 30.46 & 72.53 & 70.16 \\
1.8 & 26 & 7.70 & 26.85 & 64.89 & 64.43 \\
2 & 25 & -1.74 & 29.11 & 62.79 & 62.77 \\
2.2 & 24 & -10.18 & 32.18 & 63.96 & 63.14 \\
\hline 2.4 & 24 & & & & \\
2.6 & 24 & & & &
\end{tabular}




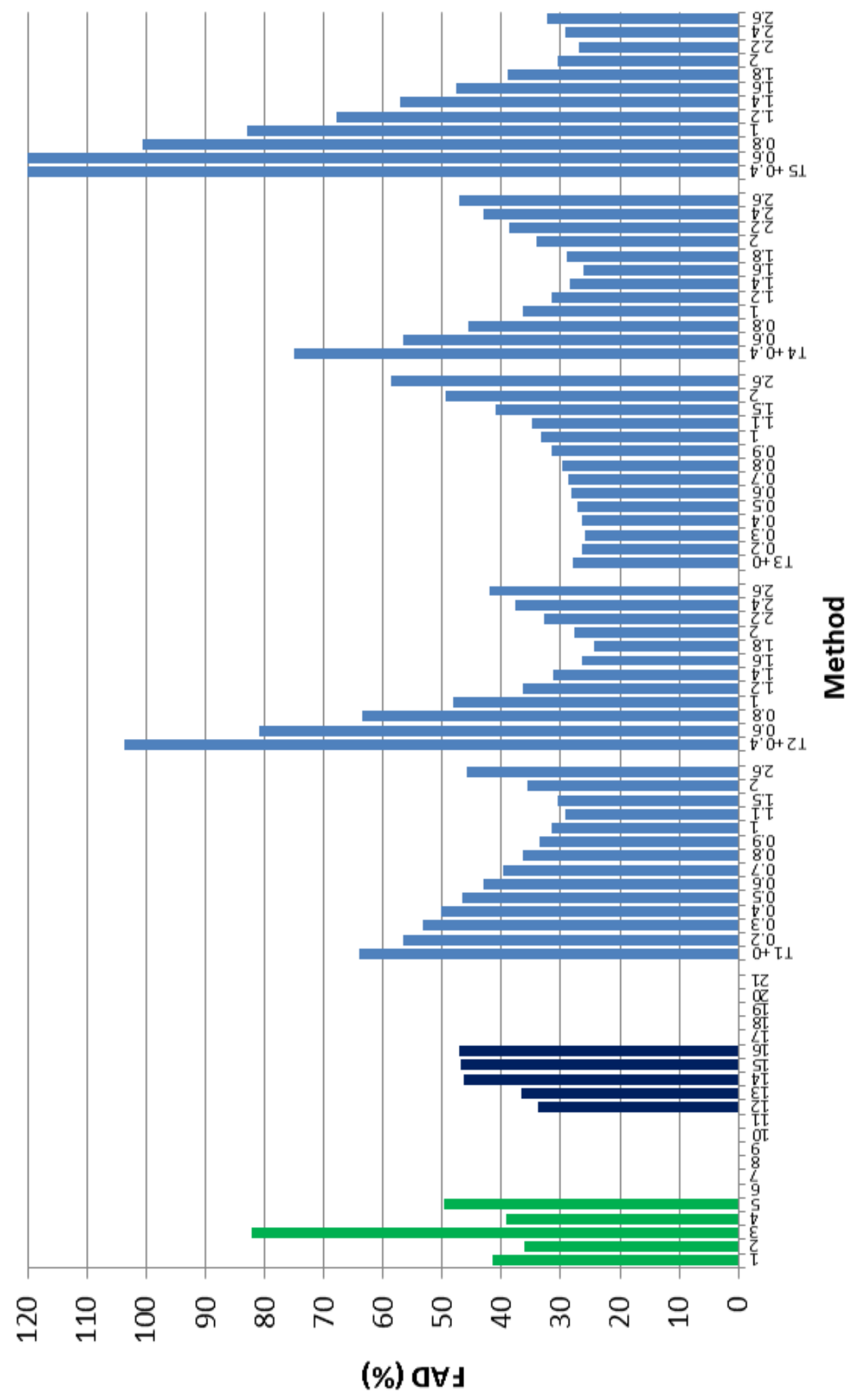

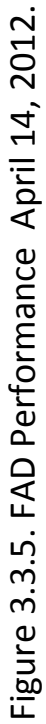




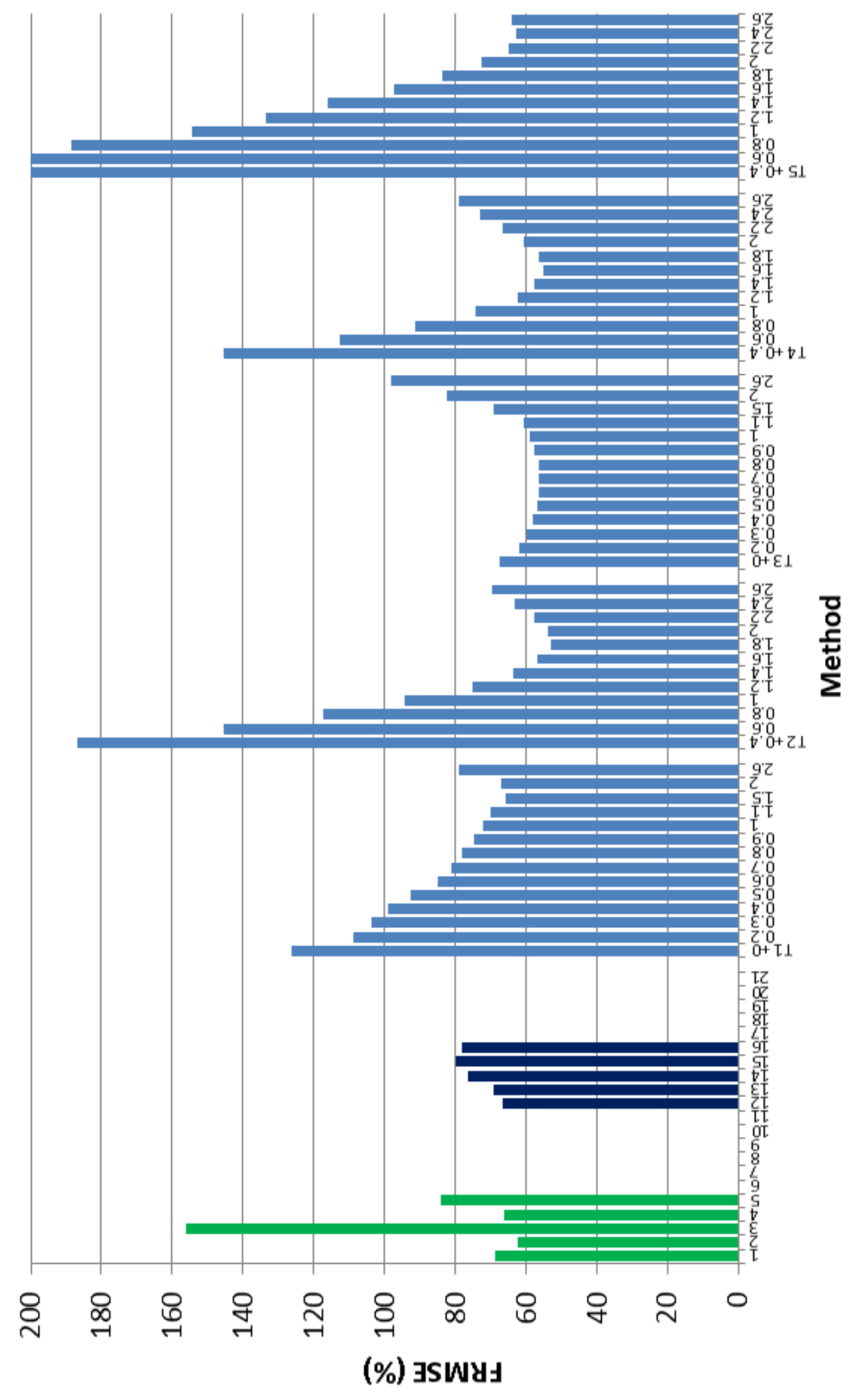

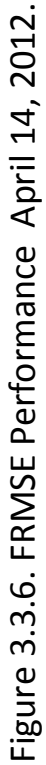




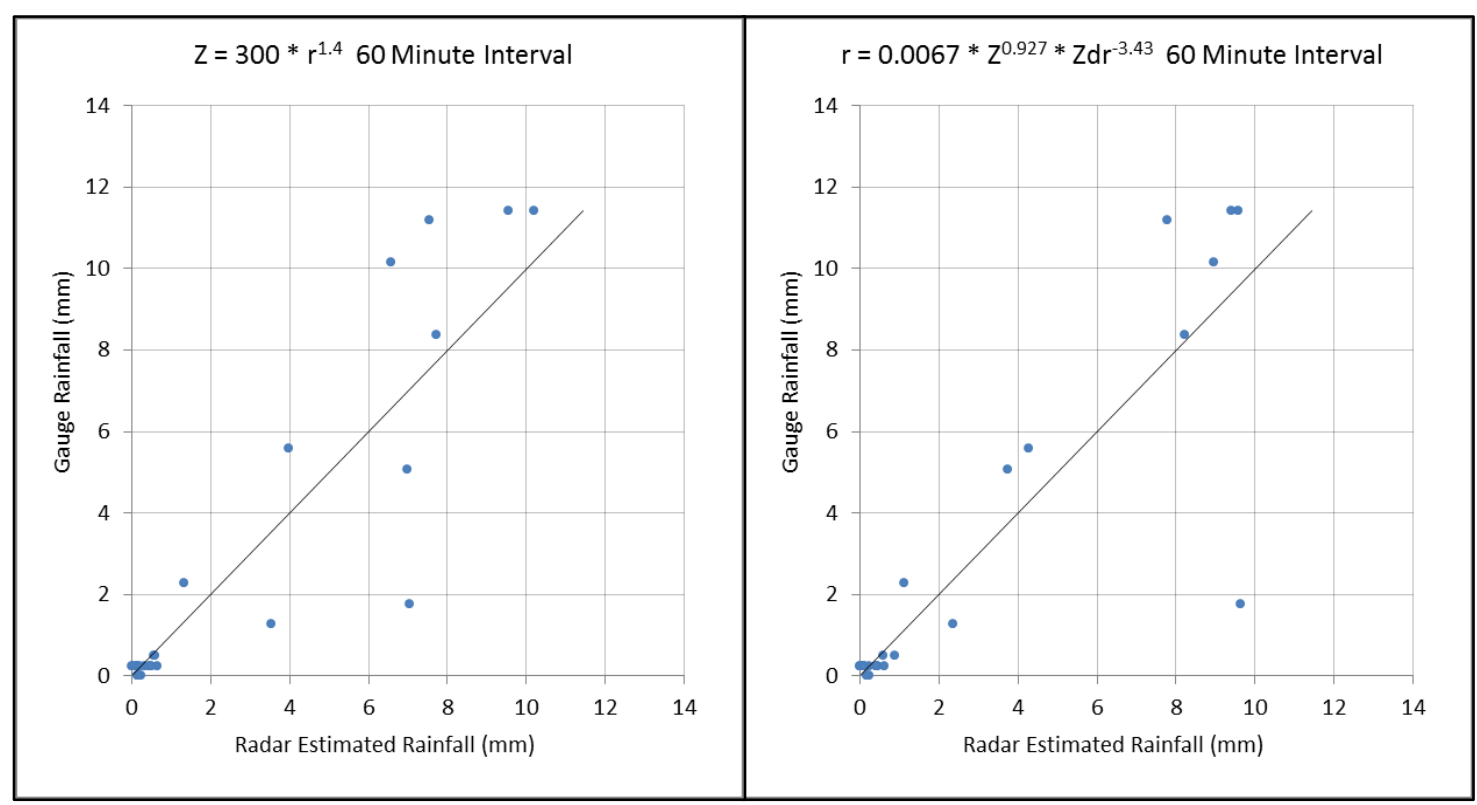

Figure 3.3.7. Radar Estimated Rainfall vs. Gauge Rainfall Scatterplots April 14, 2012. The best performers of the five conventional formula groups are shown here. 

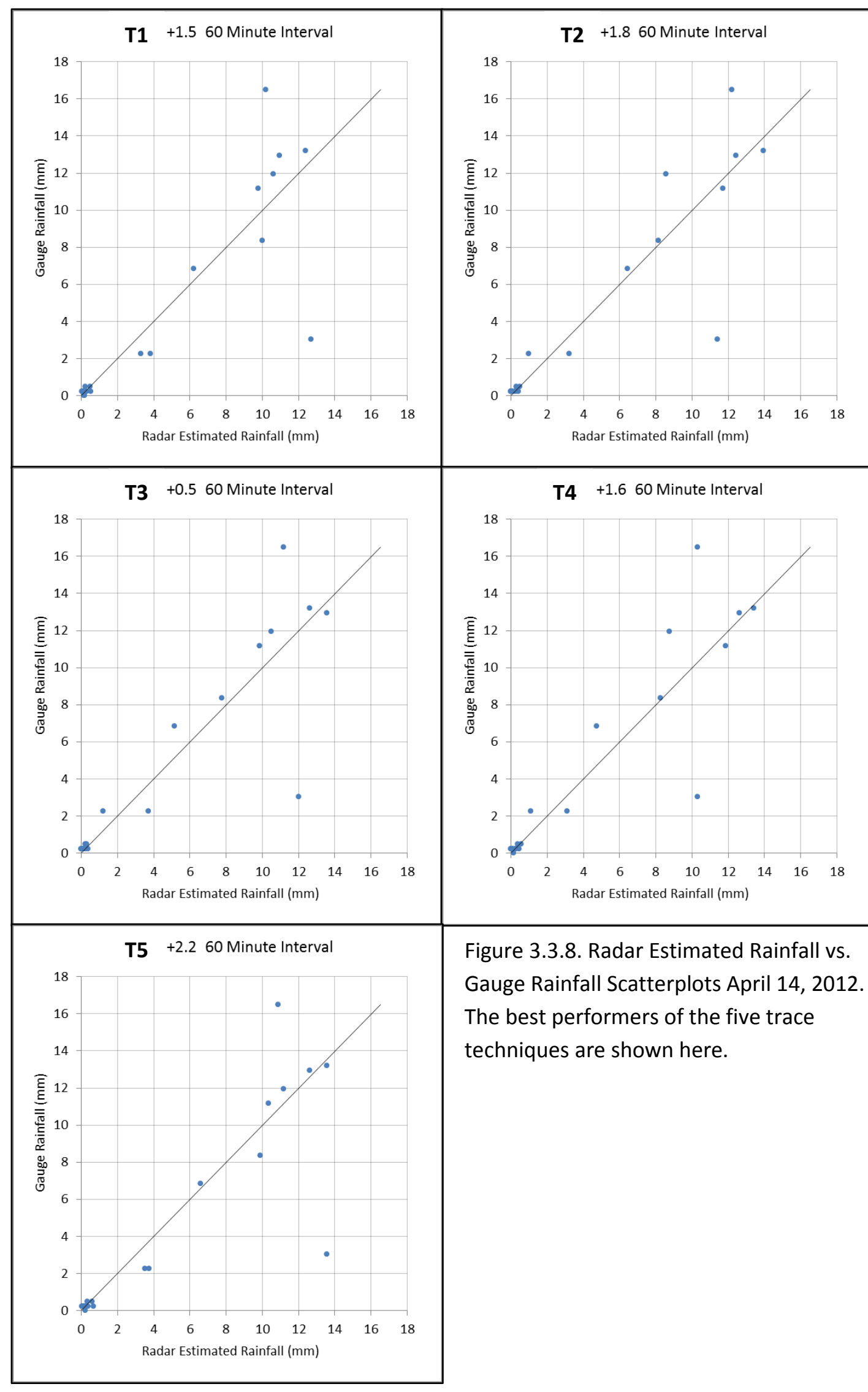

Figure 3.3.8. Radar Estimated Rainfall vs. Gauge Rainfall Scatterplots April 14, 2012. The best performers of the five trace techniques are shown here. 


\subsection{April 15, 2012}

On the $15^{\text {th }}$ of April, the system affecting the area finally began to move. Figure 3.4 .1 is an HPC surface analysis for 15 UTC of April 15, 2012. The precipitation on the $15^{\text {th }}$ occurred ahead of the cold front that would move through late in the evening of the $15^{\text {th }}$. Figure 3.4 .2 shows humidity versus height in percentile format for 10 UTC through 20 UTC of April 15. The humidity profile was not drastically different from the $14^{\text {th }}$. Figure 3.4.3 is a hodograph for the $15^{\text {th }}$ of April. The hodograph shows that a very strong southwesterly wind was present from 900 millibars and up.

\subsubsection{Conventional Formulas}

Due to extreme noise in the $K_{D P}$ data, formulas involving $K_{D P}$ were not evaluated for the precipitation events of April 13, April 14, and April 15 of 2012.

Table 3.4.1 shows the performance of the conventional techniques for this event. (5) was most effective of the formulas evaluated. Three outliers were present, the same ones from April $13(3,12$, and 13). Biases for the conventional techniques were mostly positive for this date.

\subsubsection{Tracing Techniques}

Table 3.4.2 shows the performance of the various trace techniques and their permutations for this event. Techniques T2, T4, and T5 were most effective at the $+Z_{D R}$ values of around 2 , while $\mathrm{T} 1$ and $\mathrm{T} 3$ were most effective at $+\mathrm{Z}_{\mathrm{DR}}$ values near 1 .

The overall effectiveness of the tracing techniques was similar to the conventional formulas on the $15^{\text {th }}$. 


\subsubsection{Graphical Evaluation}

Figure 3.4.4 is a histogram showing the FAD performance of each evaluated technique for this event. Figure 3.4.5 is the equivalent histogram showing FRMSE performance.

Figure 3.4.6 shows a combination of scatterplots of radar-derived rainfall versus rain gauge rainfall for the conventional formulas. The techniques illustrated were the top performers in each category. Figure 3.4.7 is identical to 3.4.6, except it depicts the best performers of the trace techniques. 


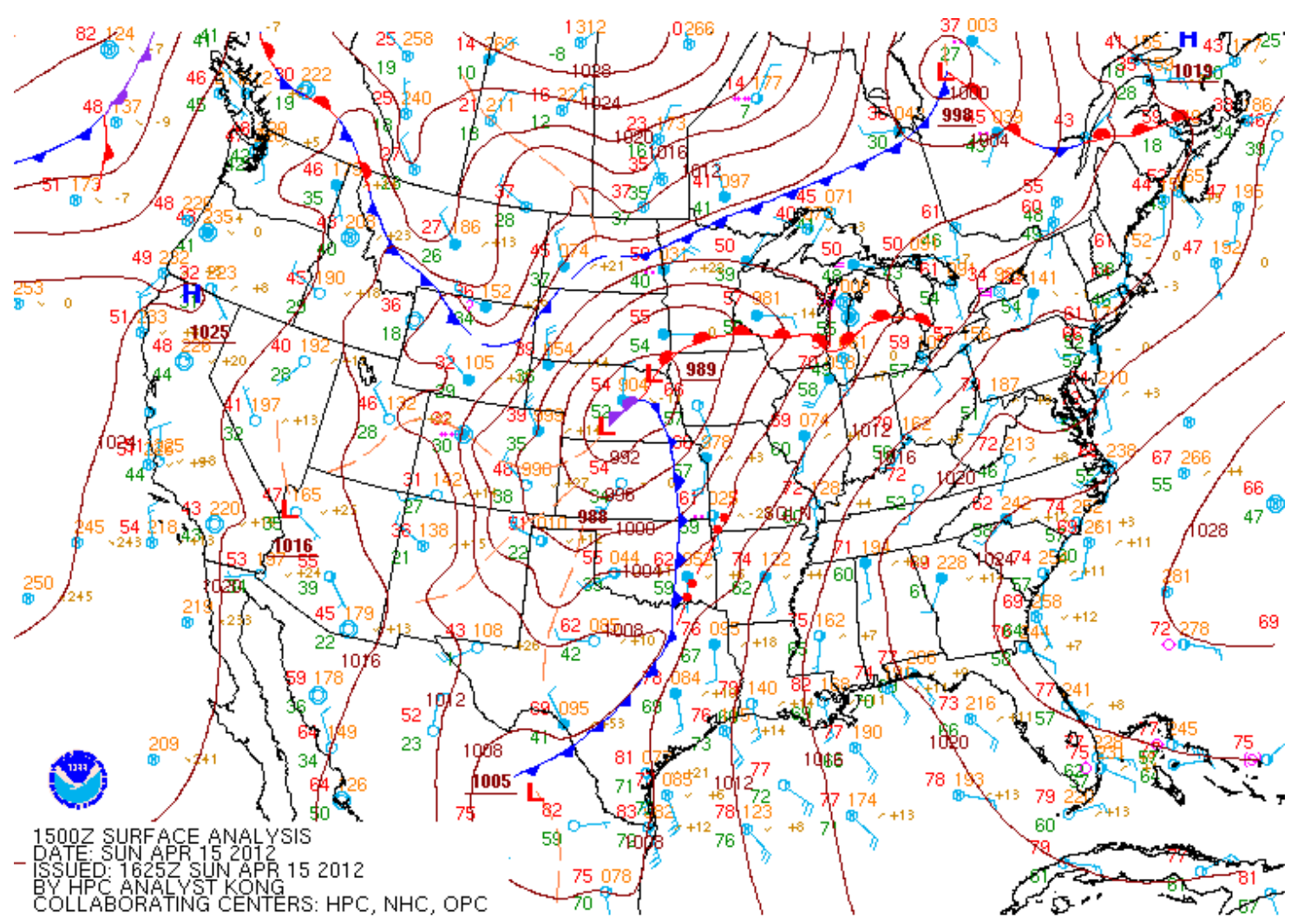

Figure 3.4.1. Surface Analysis 1500 UTC April 15, 2012.

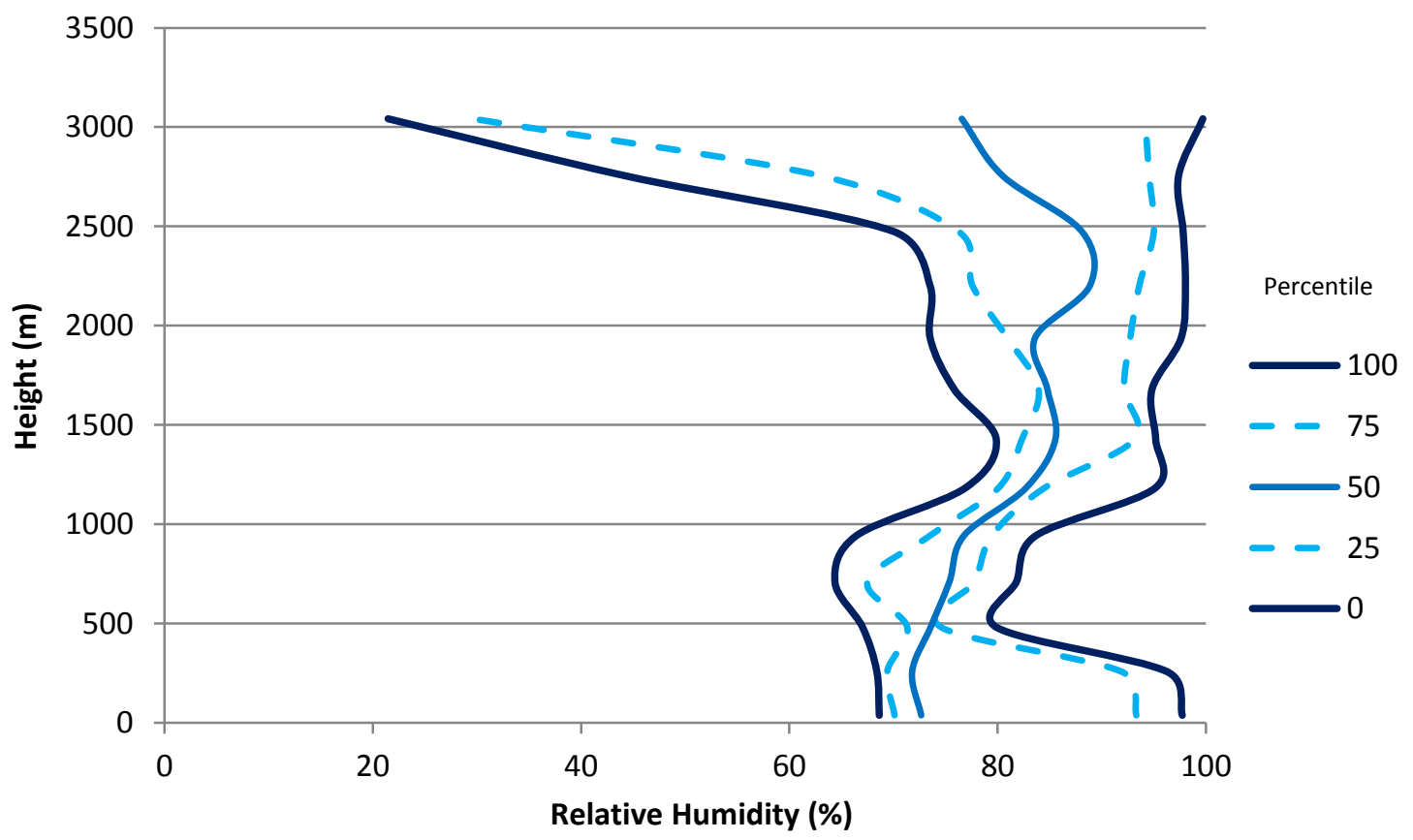

Figure 3.4.2. Relative Humidity Profile April 15, 2012. 


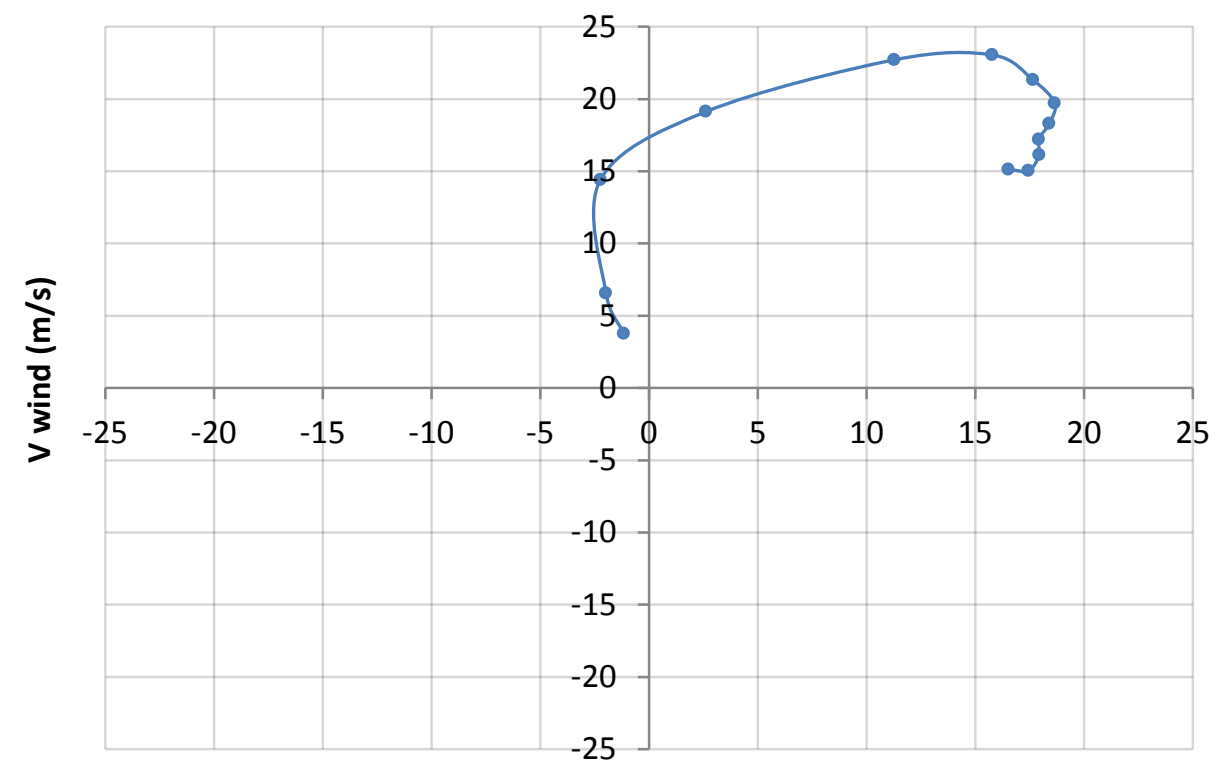

$U$ wind $(\mathrm{m} / \mathrm{s})$

$\longrightarrow$ Mean Wind

Figure 3.4.3. Hodograph April 15, 2012. 
Table 3.4.1 Conventional Techniques, April 15, 2012

\begin{tabular}{lccccc}
\hline$Z=a R^{b}$ & $\#$ & $\mathrm{FB}(\%)$ & $\mathrm{FAD}(\%)$ & $\mathrm{FRMSE}(\%)$ & $\mathrm{FSD}(\%)$ \\
\hline 1 & 27 & 27.31 & 40.66 & 50.70 & 42.72 \\
2 & 25 & 23.05 & 37.82 & 46.98 & 40.94 \\
3 & 25 & 96.74 & 105.06 & 132.01 & 89.83 \\
4 & 25 & 16.12 & 31.96 & 40.91 & 37.60 \\
5 & 24 & -18.78 & 24.57 & 35.82 & 30.50 \\
\hline$R\left(Z, Z_{D R}\right)=a Z^{b} Z_{d r}^{c}$ & & & & \\
\hline 12 & 25 & 58.55 & 73.54 & 94.92 & 74.71 \\
13 & 25 & 71.58 & 87.34 & 113.66 & 88.30 \\
14 & 25 & 20.33 & 37.57 & 48.01 & 43.49 \\
15 & 25 & 14.36 & 31.53 & 41.55 & 38.99 \\
16 & 25 & 16.34 & 34.12 & 44.31 & 41.19 \\
\hline
\end{tabular}


Table 3.4.2 Trace Techniques, April 15, 2012

\begin{tabular}{|c|c|c|c|c|c|c|}
\hline & $Z_{D R}+$ & $\#$ & $\mathrm{FB}(\%)$ & $\mathrm{FAD}(\%)$ & FRMSE(\%) & $\mathrm{FSD}(\%)$ \\
\hline \multirow[t]{14}{*}{ T1 } & 0 & 24 & 41.06 & 52.72 & 68.34 & 54.63 \\
\hline & 0.2 & 24 & 34.83 & 47.65 & 60.77 & 49.80 \\
\hline & 0.3 & 24 & 31.10 & 44.50 & 56.65 & 47.35 \\
\hline & 0.4 & 24 & 27.00 & 41.58 & 52.81 & 45.39 \\
\hline & 0.5 & 24 & 22.69 & 38.73 & 49.31 & 43.78 \\
\hline & 0.6 & 24 & 18.34 & 35.92 & 46.44 & 42.66 \\
\hline & 0.7 & 24 & 13.77 & 33.54 & 44.26 & 42.07 \\
\hline & 0.8 & 24 & 9.05 & 32.12 & 42.35 & 41.37 \\
\hline & 0.9 & 24 & 4.45 & 31.46 & 41.03 & 40.79 \\
\hline & 1 & 24 & -0.03 & 31.16 & 40.45 & 40.45 \\
\hline & 1.1 & 24 & -4.26 & 31.57 & 40.76 & 40.54 \\
\hline & 1.5 & 24 & -19.74 & 34.07 & 45.61 & 41.11 \\
\hline & 2 & 24 & -35.79 & 39.06 & 55.97 & 43.03 \\
\hline & 2.6 & 24 & -49.99 & 49.99 & 69.75 & 48.63 \\
\hline \multirow[t]{12}{*}{$\mathrm{T} 2$} & 0.4 & 27 & 206.38 & 210.00 & 313.31 & 235.74 \\
\hline & 0.6 & 26 & 158.24 & 162.52 & 240.54 & 181.16 \\
\hline & 0.8 & 26 & 118.02 & 125.62 & 183.49 & 140.51 \\
\hline & 1 & 24 & 85.05 & 95.47 & 133.13 & 102.42 \\
\hline & 1.2 & 24 & 60.59 & 74.01 & 101.54 & 81.48 \\
\hline & 1.4 & 24 & 41.42 & 57.49 & 78.12 & 66.23 \\
\hline & 1.6 & 24 & 25.89 & 44.65 & 60.95 & 55.18 \\
\hline & 1.8 & 24 & 12.71 & 34.54 & 48.80 & 47.11 \\
\hline & 2 & 24 & 1.28 & 29.07 & 41.45 & 41.43 \\
\hline & 2.2 & 24 & -8.50 & 25.57 & 39.10 & 38.17 \\
\hline & 2.4 & 24 & -16.99 & 25.77 & 40.60 & 36.87 \\
\hline & 2.6 & 24 & -24.41 & 28.05 & 44.35 & 37.03 \\
\hline \multirow[t]{14}{*}{ T3 } & 0 & 24 & 58.34 & 71.54 & 90.62 & 69.34 \\
\hline & 0.2 & 24 & 49.51 & 63.66 & 81.00 & 64.10 \\
\hline & 0.3 & 24 & 44.75 & 59.30 & 75.76 & 61.13 \\
\hline & 0.4 & 24 & 39.78 & 54.76 & 70.43 & 58.13 \\
\hline & 0.5 & 24 & 34.60 & 50.04 & 65.12 & 55.17 \\
\hline & 0.6 & 24 & 29.29 & 45.43 & 59.94 & 52.29 \\
\hline & 0.7 & 24 & 23.96 & 41.23 & 55.11 & 49.63 \\
\hline & 0.8 & 24 & 18.69 & 37.58 & 50.77 & 47.21 \\
\hline & 0.9 & 24 & 13.50 & 34.68 & 47.12 & 45.14 \\
\hline & 1 & 24 & 8.45 & 32.65 & 44.29 & 43.48 \\
\hline & 1.1 & 24 & 3.58 & 30.77 & 42.37 & 42.22 \\
\hline & 1.5 & 24 & -13.44 & 27.58 & 42.92 & 40.77 \\
\hline & 2 & 24 & -30.35 & 35.36 & 53.12 & 43.60 \\
\hline & 2.6 & 24 & -45.69 & 47.25 & 67.04 & 49.05 \\
\hline
\end{tabular}


Table 3.4.2 Trace Techniques, April 15, 2012 (Continued)

\begin{tabular}{|c|c|c|c|c|c|c|}
\hline & $Z_{D R}+$ & $\#$ & $\mathrm{FB}(\%)$ & $\mathrm{FAD}(\%)$ & FRMSE(\%) & $\mathrm{FSD}(\%)$ \\
\hline \multirow[t]{12}{*}{ T4 } & 0.4 & 24 & 202.72 & 206.41 & 265.93 & 172.12 \\
\hline & 0.6 & 24 & 155.47 & 161.45 & 207.18 & 136.93 \\
\hline & 0.8 & 24 & 115.78 & 124.93 & 157.46 & 106.72 \\
\hline & 1 & 24 & 83.82 & 95.44 & 118.39 & 83.62 \\
\hline & 1.2 & 24 & 59.60 & 73.20 & 90.06 & 67.52 \\
\hline & 1.4 & 24 & 40.61 & 55.82 & 69.53 & 56.44 \\
\hline & 1.6 & 24 & 25.21 & 42.64 & 55.31 & 49.23 \\
\hline & 1.8 & 24 & 12.14 & 34.58 & 46.29 & 44.67 \\
\hline & 2 & 24 & 0.78 & 30.05 & 42.06 & 42.05 \\
\hline & 2.2 & 24 & -8.95 & 27.82 & 42.13 & 41.17 \\
\hline & 2.4 & 24 & -17.38 & 28.59 & 45.00 & 41.50 \\
\hline & 2.6 & 24 & -24.76 & 31.76 & 49.29 & 42.62 \\
\hline \multirow[t]{12}{*}{ T5 } & 0.4 & 26 & 180.34 & 183.01 & 232.50 & 146.75 \\
\hline & 0.6 & 26 & 139.84 & 143.02 & 184.00 & 119.60 \\
\hline & 0.8 & 26 & 103.41 & 107.37 & 142.10 & 97.46 \\
\hline & 1 & 24 & 73.46 & 78.89 & 104.34 & 74.10 \\
\hline & 1.2 & 24 & 50.51 & 58.38 & 80.66 & 62.89 \\
\hline & 1.4 & 24 & 32.08 & 44.14 & 63.34 & 54.62 \\
\hline & 1.6 & 24 & 16.53 & 34.48 & 49.34 & 46.49 \\
\hline & 1.8 & 24 & 3.85 & 31.07 & 41.83 & 41.65 \\
\hline & 2 & 24 & -6.83 & 30.24 & 39.87 & 39.28 \\
\hline & 2.2 & 24 & -15.94 & 32.05 & 41.93 & 38.79 \\
\hline & 2.4 & 24 & -23.82 & 34.16 & 46.17 & 39.54 \\
\hline & 2.6 & 24 & -30.71 & 36.44 & 51.28 & 41.06 \\
\hline
\end{tabular}




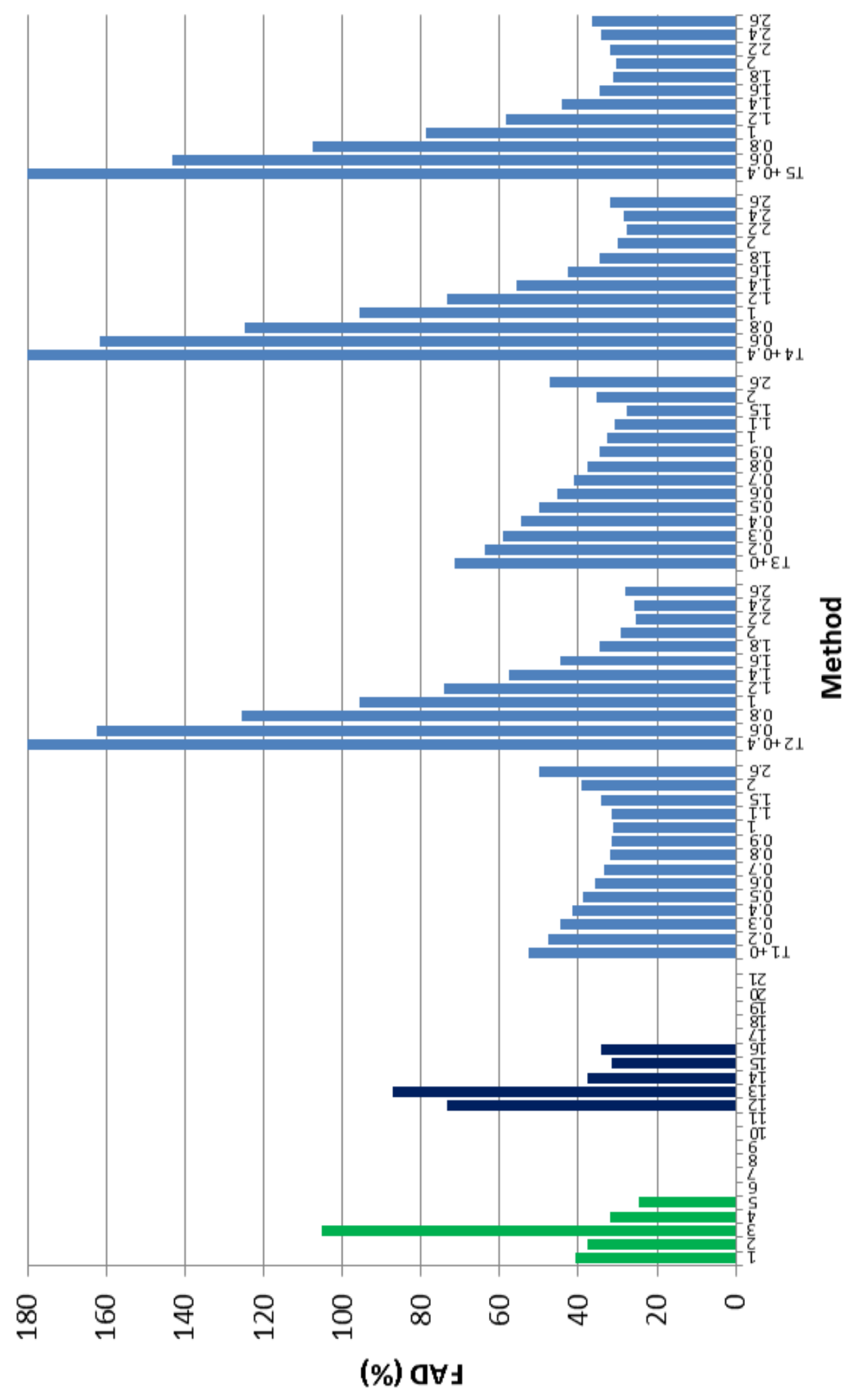

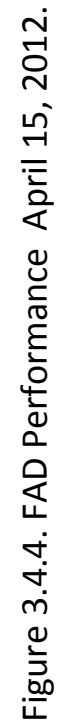




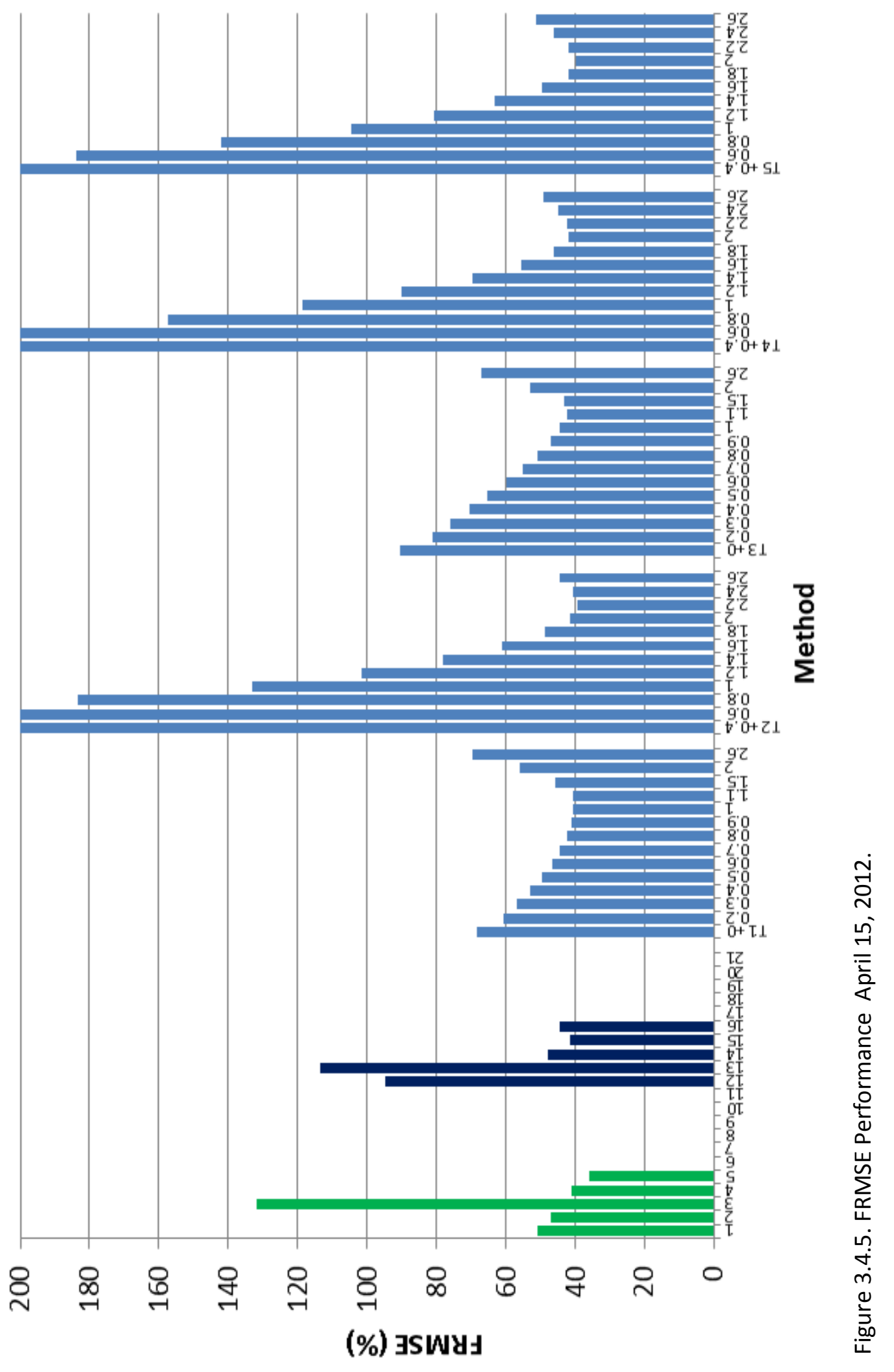




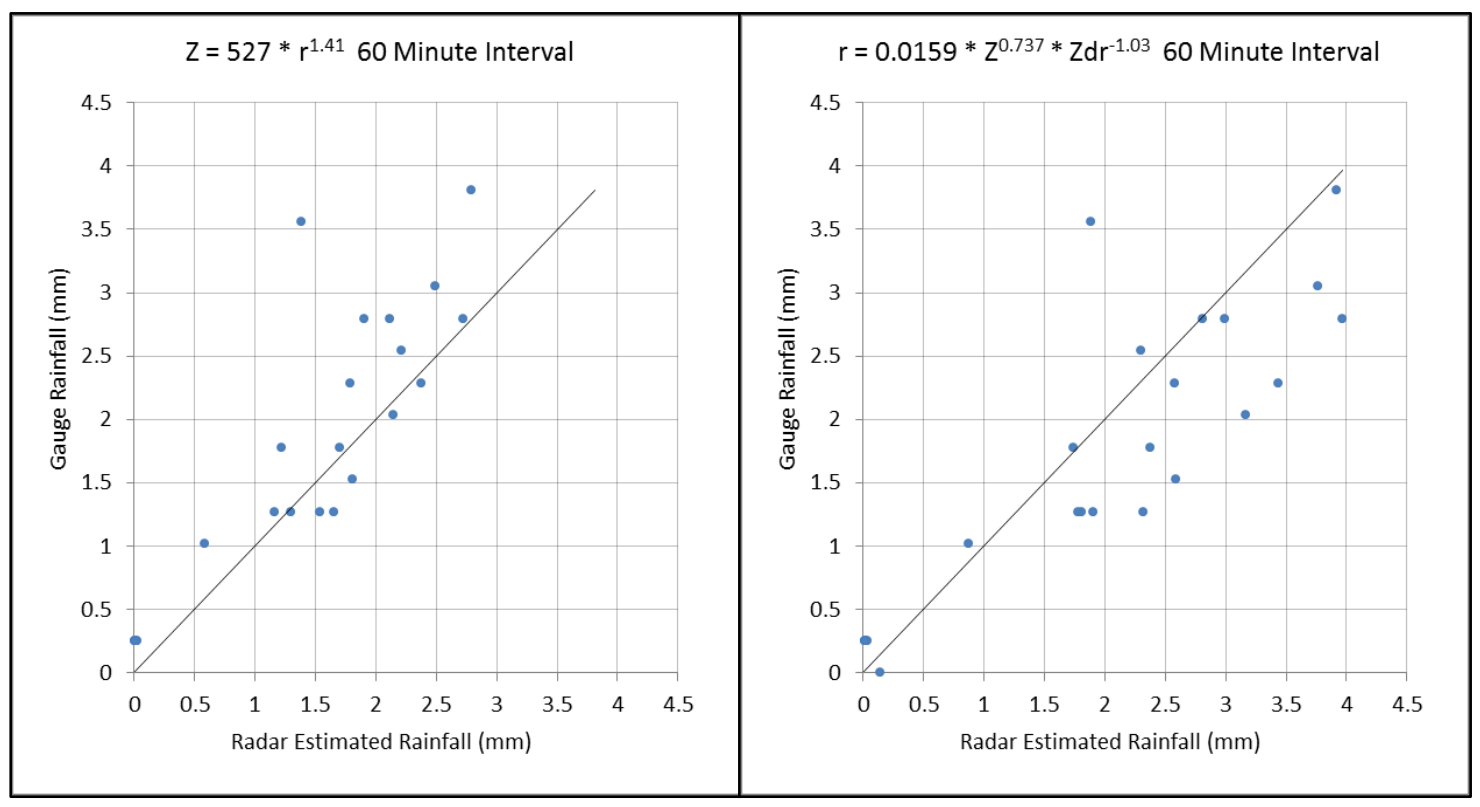

Figure 3.4.6. Radar Estimated Rainfall vs. Gauge Rainfall Scatterplots April 15, 2012. The best performers of the five conventional formula groups are shown here. 

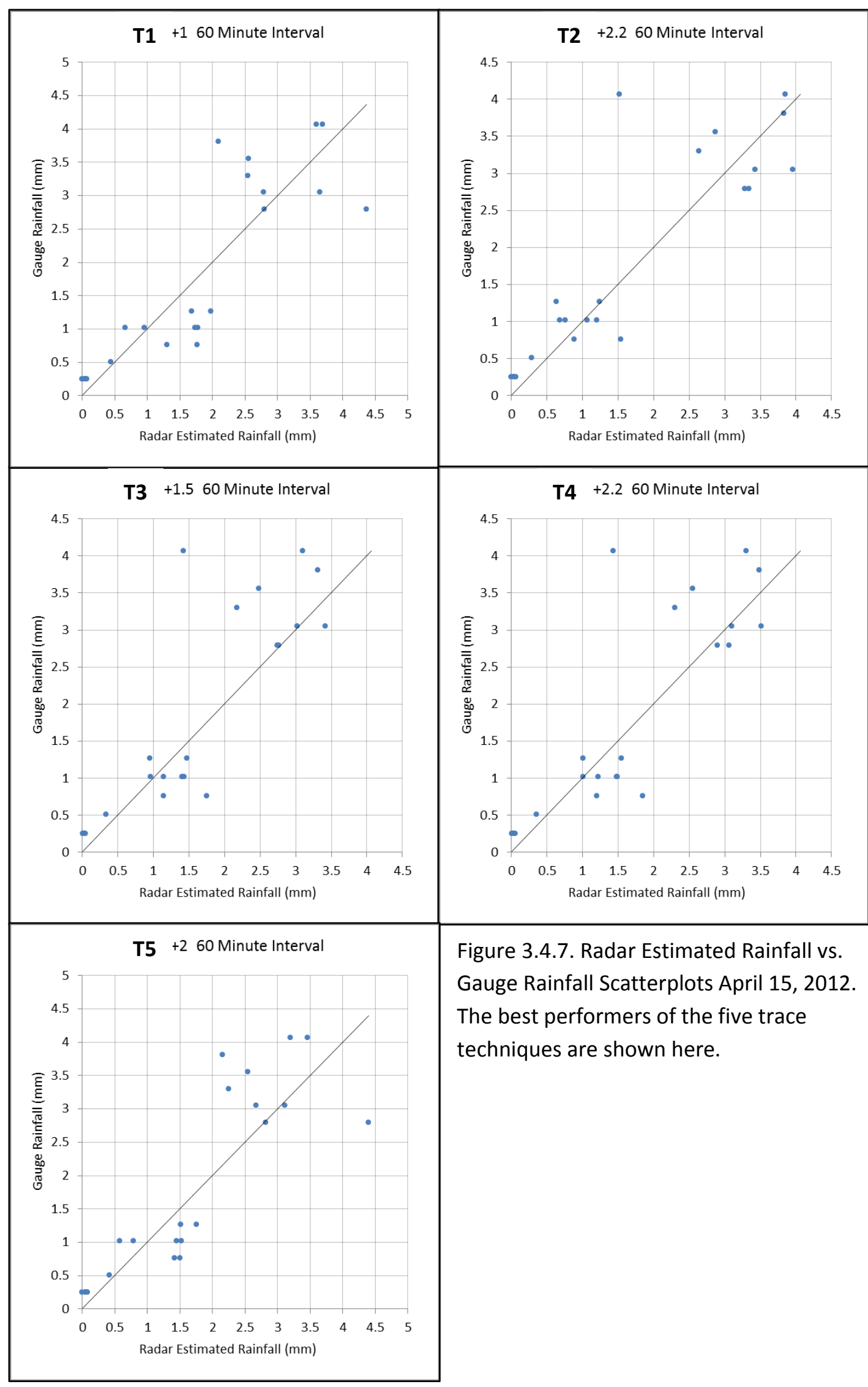

Figure 3.4.7. Radar Estimated Rainfall vs. Gauge Rainfall Scatterplots April 15, 2012. The best performers of the five trace techniques are shown here. 


\subsection{June 16, 2012}

The event on the $16^{\text {th }}$ of June occurred when a squall line passed through Central Missouri ahead of a cold front. Figure 3.5.1 is an HPC surface analysis for 00 UTC of June 17, 2012. The precipitation on this day came from several strong thunderstorms moving through the area. Figure 3.5.2 shows humidity versus height in percentile format for 18 UTC of June 16 through 03 UTC of June 17. The atmosphere was relatively dry for this event, especially in the lower levels. Figure 3.5 .3 is a hodograph for the $16^{\text {th }}$ of June. The hodograph shows a weak southwesterly wind at the surface turning more in a west southwesterly direction higher up. Figure 3.5.4 is a four panel radar image from KLSX for 0021 UTC of June 17.

\subsubsection{Conventional Formulas}

Table 3.5.1 shows the performance of the conventional formulas for this event. (19) was most effective of the conventional formulas evaluated. The simple Z-R formulas performed relatively poorly. The $\mathrm{K}_{\mathrm{DP}}$-reliant formulas had their best showing in this event, performing better than the Z-R formulas at several points. One outlier, (3), was present. Biases for the conventional techniques were mostly positive for this date.

\subsubsection{Tracing Techniques}

Table 3.5.2 shows the performance of the various trace techniques and their permutations for this event. The no-evaporation techniques were most effective at $+Z_{D R}$ values of around 2 , while $T 1$ and $T 3$ were most effective at $+Z_{D R}$ values from 1 to 1.5 . 
The optimal T3 and T5 techniques performed better than any conventional formulas for this event. The other trace techniques performed similarly to the conventional formulas. The evaporation techniques exhibited negative biases at all $Z_{D R}$ adjustments.

\subsubsection{Graphical Evaluation}

Figure 3.5.5 is a histogram showing the FAD performance of each evaluated technique for this event. Figure 3.5.6 is the equivalent histogram showing FRMSE performance.

Figure 3.5.7 shows a combination of scatterplots of radar-derived rainfall versus rain gauge rainfall for the conventional formulas. The techniques illustrated were the top performers in each category. Figure 3.5.8 is identical to 3.5.7, except it depicts the best performers of the trace techniques. 


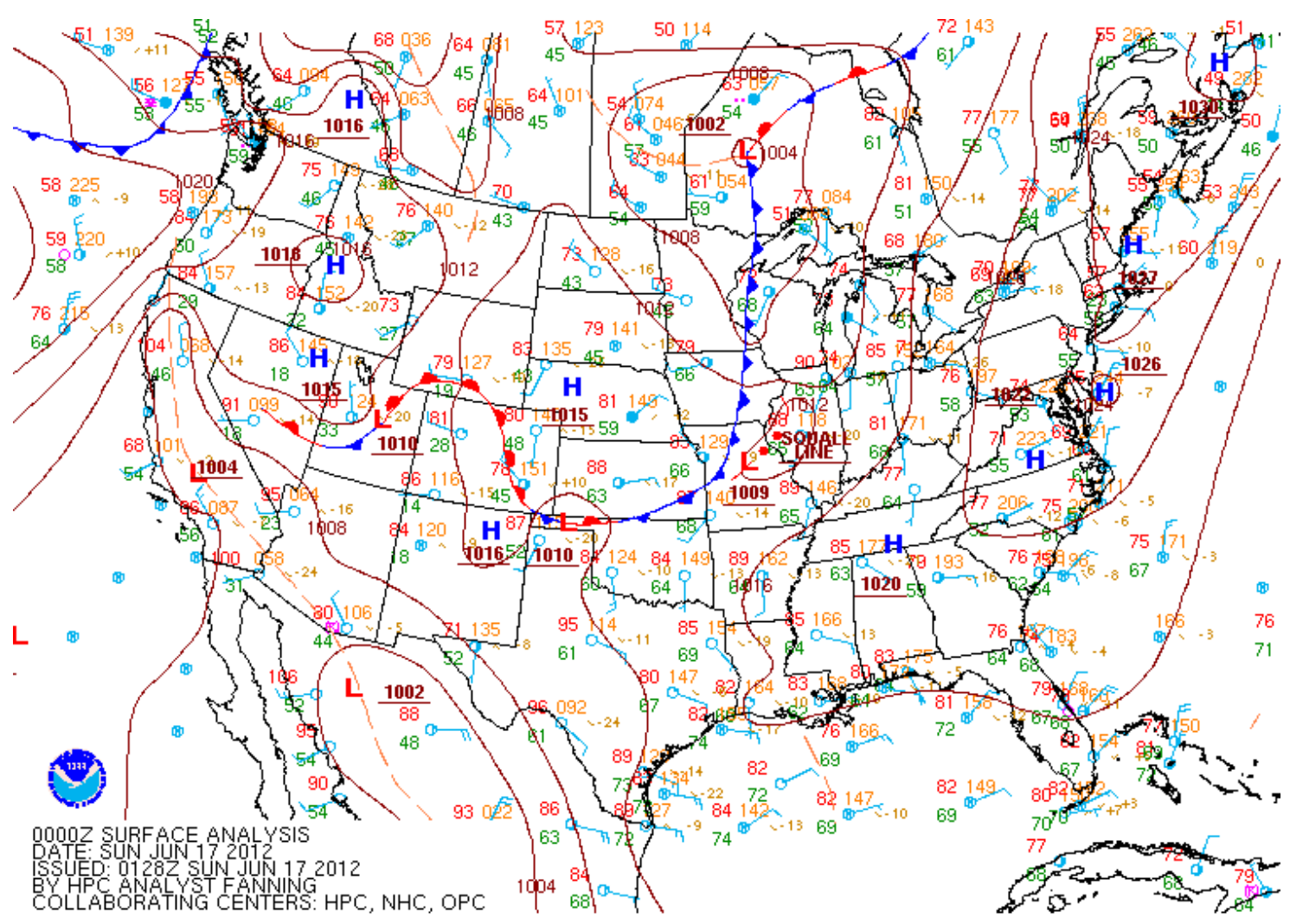

Figure 3.5.1. Surface Analysis 0000 UTC June 17, 2012.

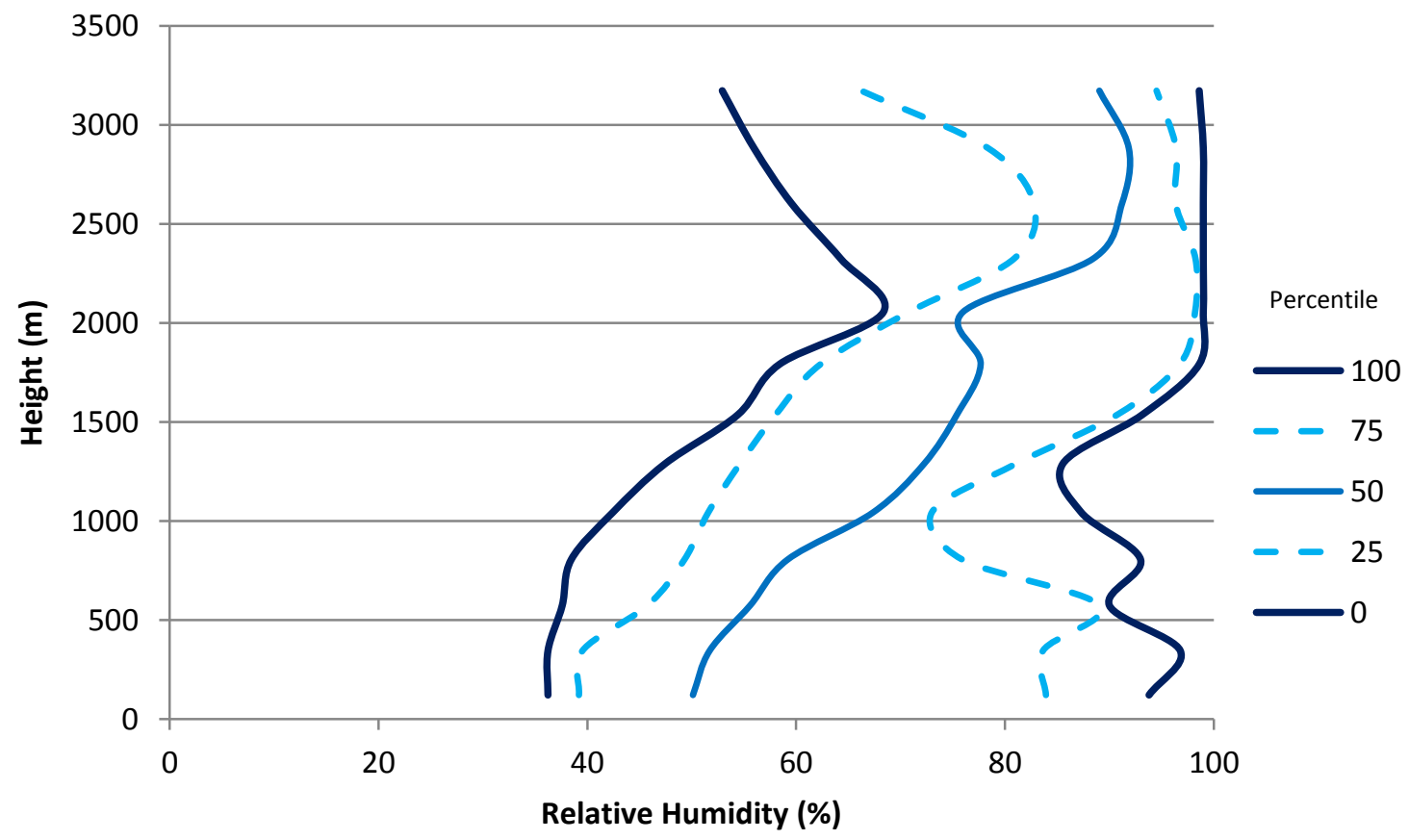

Figure 3.5.2. Relative Humidity Profile June 16, 2012. 


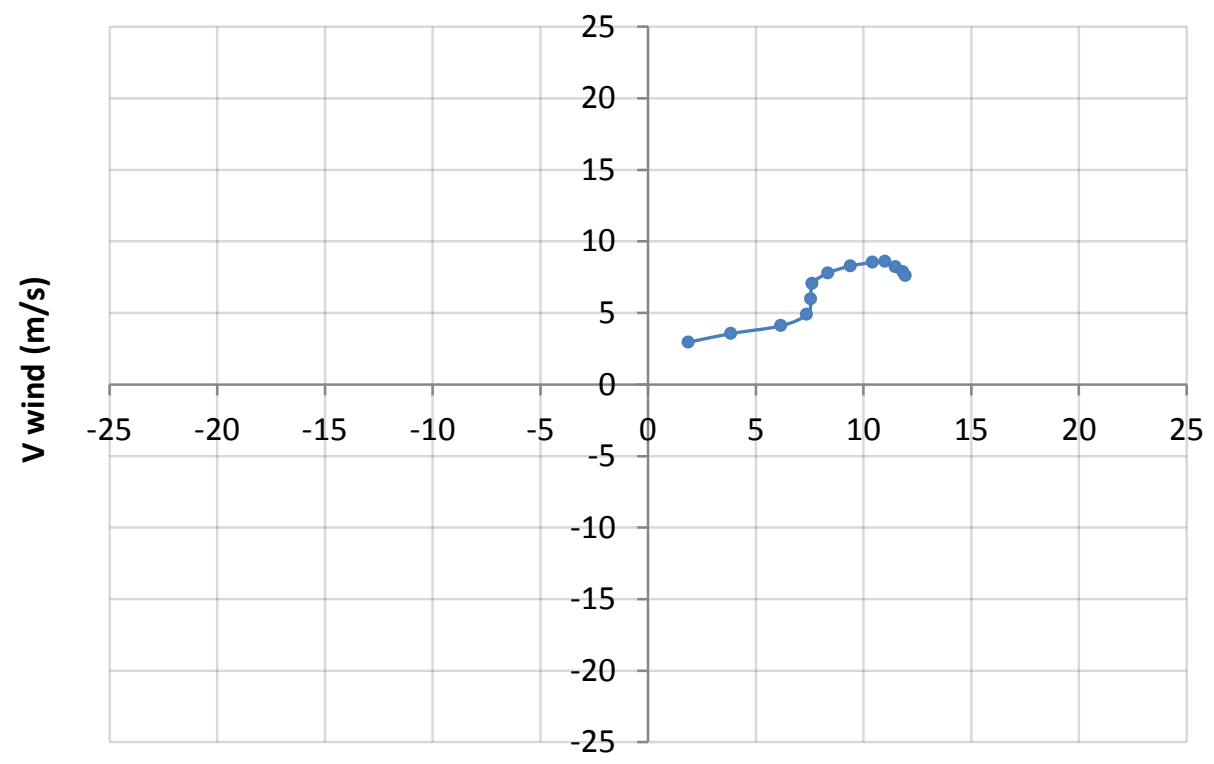

$U$ wind $(\mathrm{m} / \mathrm{s})$

Figure 3.5.3. Hodograph June 16, 2012. 


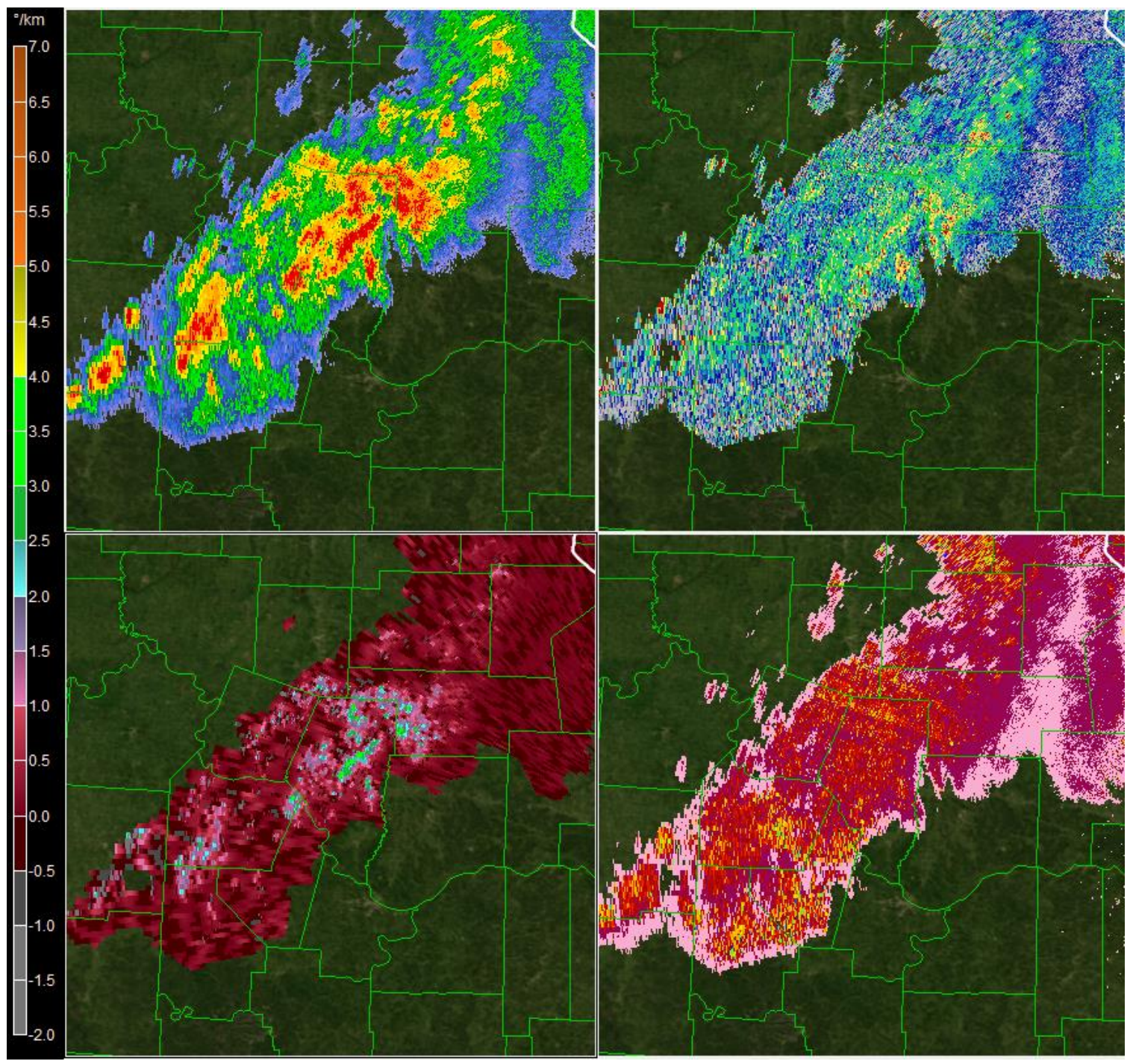

Figure 3.5.4. Four Panel Radar Image for 0021 UTC June 17, 2012. The top left is reflectivity $(Z)$, top right is differential reflectivity $\left(Z_{D R}\right)$, bottom left is specific phase differential $\left(K_{D P}\right)$, and bottom right is correlation coefficient. The scale on the left corresponds to the bottom left image $\left(K_{D P}\right)$. The elevation angle used here was $0.5^{\circ}$. 
Table 3.5.1 Conventional Techniques, June 16, 2012

\begin{tabular}{|c|c|c|c|c|c|}
\hline$Z=a R^{b}$ & $\#$ & $\mathrm{FB}(\%)$ & $\mathrm{FAD}(\%)$ & FRMSE(\%) & FSD(\%) \\
\hline 1 & 30 & -0.94 & 49.59 & 72.92 & 72.92 \\
\hline 2 & 30 & 15.59 & 41.62 & 69.31 & 67.53 \\
\hline 3 & 30 & 139.15 & 139.81 & 219.40 & 169.63 \\
\hline 4 & 30 & 4.65 & 45.62 & 69.84 & 69.69 \\
\hline 5 & 30 & -24.30 & 56.86 & 88.30 & 84.89 \\
\hline \multicolumn{6}{|c|}{$R\left(K_{D P}\right)=a\left|K_{D P}\right|^{b} \operatorname{sign}\left(K_{D P}\right)$} \\
\hline 6 & 33 & 26.31 & 45.12 & 66.54 & 61.11 \\
\hline 7 & 33 & 36.57 & 54.13 & 77.57 & 68.41 \\
\hline 8 & 33 & 33.07 & 52.63 & 74.20 & 66.43 \\
\hline 9 & 33 & 10.27 & 37.43 & 57.47 & 56.55 \\
\hline 10 & 33 & 26.34 & 45.64 & 66.78 & 61.37 \\
\hline 11 & 33 & 19.38 & 41.69 & 61.62 & 58.49 \\
\hline \multicolumn{6}{|c|}{$R\left(Z, Z_{D R}\right)=a Z^{b} Z_{d r}^{c}$} \\
\hline 12 & 30 & -39.27 & 65.55 & 109.07 & 101.75 \\
\hline 13 & 30 & -49.94 & 71.09 & 122.93 & 112.33 \\
\hline 14 & 30 & -39.79 & 67.28 & 109.83 & 102.37 \\
\hline 15 & 30 & -34.25 & 65.37 & 104.09 & 98.30 \\
\hline 16 & 30 & -39.84 & 67.41 & 109.90 & 102.42 \\
\hline \multicolumn{6}{|c|}{$R\left(K_{D P}, Z_{D R}\right)=a\left|K_{D P}\right|^{b} Z_{d r}^{c} \operatorname{sign}\left(K_{D P}\right)$} \\
\hline 17 & 32 & 25.82 & 53.39 & 70.90 & 66.03 \\
\hline 18 & 33 & 26.40 & 63.87 & 82.58 & 78.25 \\
\hline 19 & 33 & 10.74 & 39.97 & 58.89 & 57.90 \\
\hline 20 & 32 & 24.81 & 48.47 & 66.82 & 62.04 \\
\hline \multicolumn{6}{|l|}{ Synthetic } \\
\hline 21 & 32 & 24.00 & 38.73 & 62.58 & 57.80 \\
\hline
\end{tabular}


Table 3.5.2 Trace Techniques, June 16, 2012

\begin{tabular}{|c|c|c|c|c|c|c|}
\hline & $Z_{D R}+$ & $\#$ & $\mathrm{FB}(\%)$ & FAD(\%) & FRMSE(\%) & $\mathrm{FSD}(\%)$ \\
\hline \multirow[t]{14}{*}{ T1 } & 0 & 23 & -22.76 & 35.60 & 57.84 & 53.17 \\
\hline & 0.2 & 23 & -23.92 & 35.93 & 58.20 & 53.05 \\
\hline & 0.3 & 24 & -24.32 & 36.14 & 59.59 & 54.41 \\
\hline & 0.4 & 23 & -24.79 & 36.16 & 58.44 & 52.92 \\
\hline & 0.5 & 23 & -25.06 & 36.18 & 58.43 & 52.78 \\
\hline & 0.6 & 23 & -25.20 & 36.09 & 58.27 & 52.54 \\
\hline & 0.7 & 22 & -25.26 & 35.82 & 56.66 & 50.72 \\
\hline & 0.8 & 22 & -25.07 & 35.60 & 56.13 & 50.22 \\
\hline & 0.9 & 22 & -24.71 & 35.27 & 55.40 & 49.59 \\
\hline & 1 & 23 & -24.25 & 34.80 & 55.69 & 50.13 \\
\hline & 1.1 & 23 & -24.14 & 34.25 & 54.85 & 49.25 \\
\hline & 1.5 & 23 & -25.29 & 33.04 & 53.78 & 47.47 \\
\hline & 2 & 22 & -35.63 & 41.23 & 66.01 & 55.57 \\
\hline & 2.6 & 21 & -48.50 & 52.07 & 82.53 & 66.78 \\
\hline \multirow[t]{12}{*}{$\mathrm{T} 2$} & 0.4 & 30 & 63.69 & 66.03 & 111.31 & 91.29 \\
\hline & 0.6 & 29 & 59.37 & 62.09 & 104.29 & 85.75 \\
\hline & 0.8 & 29 & 58.21 & 60.97 & 101.88 & 83.62 \\
\hline & 1 & 29 & 55.39 & 58.29 & 95.03 & 77.22 \\
\hline & 1.2 & 29 & 49.06 & 52.12 & 85.24 & 69.70 \\
\hline & 1.4 & 29 & 41.83 & 45.02 & 75.94 & 63.38 \\
\hline & 1.6 & 29 & 33.39 & 38.88 & 67.32 & 58.46 \\
\hline & 1.8 & 29 & 23.39 & 35.44 & 59.31 & 54.50 \\
\hline & 2 & 29 & 13.08 & 34.85 & 55.28 & 53.71 \\
\hline & 2.2 & 29 & 3.61 & 36.68 & 55.90 & 55.78 \\
\hline & 2.4 & 29 & -4.89 & 39.29 & 59.77 & 59.57 \\
\hline & 2.6 & 28 & -12.63 & 42.65 & 64.38 & 63.13 \\
\hline \multirow[t]{14}{*}{ T3 } & 0 & 26 & -10.76 & 34.53 & 52.66 & 51.55 \\
\hline & 0.2 & 27 & -10.43 & 34.94 & 53.21 & 52.18 \\
\hline & 0.3 & 27 & -10.17 & 34.76 & 52.45 & 51.45 \\
\hline & 0.4 & 26 & -9.80 & 34.30 & 50.49 & 49.52 \\
\hline & 0.5 & 26 & -9.14 & 33.69 & 49.27 & 48.41 \\
\hline & 0.6 & 26 & -8.25 & 32.86 & 47.84 & 47.12 \\
\hline & 0.7 & 27 & -7.08 & 31.85 & 47.03 & 46.50 \\
\hline & 0.8 & 27 & -5.81 & 30.49 & 45.04 & 44.67 \\
\hline & 0.9 & 26 & -5.01 & 29.14 & 42.39 & 42.09 \\
\hline & 1 & 26 & -4.94 & 28.29 & 40.97 & 40.67 \\
\hline & 1.1 & 26 & -5.78 & 28.38 & 40.77 & 40.36 \\
\hline & 1.5 & 26 & -11.68 & 30.98 & 45.41 & 43.89 \\
\hline & 2 & 26 & -24.62 & 39.02 & 61.47 & 56.32 \\
\hline & 2.6 & 25 & -39.60 & 50.47 & 81.24 & 70.93 \\
\hline
\end{tabular}


Table 3.5.2 Trace Techniques, June 16, 2012 (Continued)

\begin{tabular}{ccccccc}
\hline & $Z_{D R}+$ & $\#$ & $\mathrm{FB}(\%)$ & $\mathrm{FAD}(\%)$ & $\mathrm{FRMSE}(\%)$ & $\mathrm{FSD}(\%)$ \\
\hline T4 & 0.4 & 30 & 64.06 & 64.25 & 110.03 & 89.46 \\
& 0.6 & 30 & 59.87 & 60.67 & 105.56 & 86.94 \\
0.8 & 30 & 58.77 & 60.11 & 104.09 & 85.91 \\
& 29 & 55.92 & 57.69 & 95.98 & 78.00 \\
& 29 & 49.61 & 51.71 & 86.09 & 70.35 \\
& 28 & 42.32 & 44.69 & 75.21 & 62.18 \\
& 1.4 & 33.88 & 37.55 & 66.21 & 56.88 \\
& 28 & 23.87 & 33.17 & 57.30 & 52.09 \\
& 28 & 13.54 & 31.93 & 52.25 & 50.46 \\
& 28 & 4.05 & 34.04 & 52.12 & 51.96 \\
& 28 & -4.47 & 35.95 & 55.62 & 55.44 \\
& 28 & -12.17 & 39.56 & 61.25 & 60.03 \\
\hline T5 & 28 & 35.36 & 40.77 & 72.74 & 63.56 \\
& 2.4 & 28 & 24.63 & 36.71 & 64.54 & 57.84 \\
& 0.4 & 31 & 34.05 & 60.24 & 54.99 \\
0.6 & 30 & 22.52 & 32.22 & 56.58 & 51.90 \\
0.8 & 30 & 20.37 & 30.32 & 52.53 & 48.42 \\
1 & 29 & 17.70 & 28.10 & 47.38 & 43.95 \\
1.2 & 29 & 12.58 & 24.68 & 43.37 & 41.50 \\
1.4 & 28 & 4.65 & 22.05 & 41.61 & 41.34 \\
1.6 & 28 & -4.07 & 26.39 & 44.45 & 44.26 \\
1.8 & 28 & -12.15 & 31.00 & 50.95 & 49.48 \\
2 & 28 & -19.38 & 35.12 & 58.80 & 55.52 \\
2.2 & 28 & -25.89 & 39.36 & 66.94 & 61.73 \\
\hline 2.4 & 28 & & & & \\
2.6 & 28 & & & &
\end{tabular}




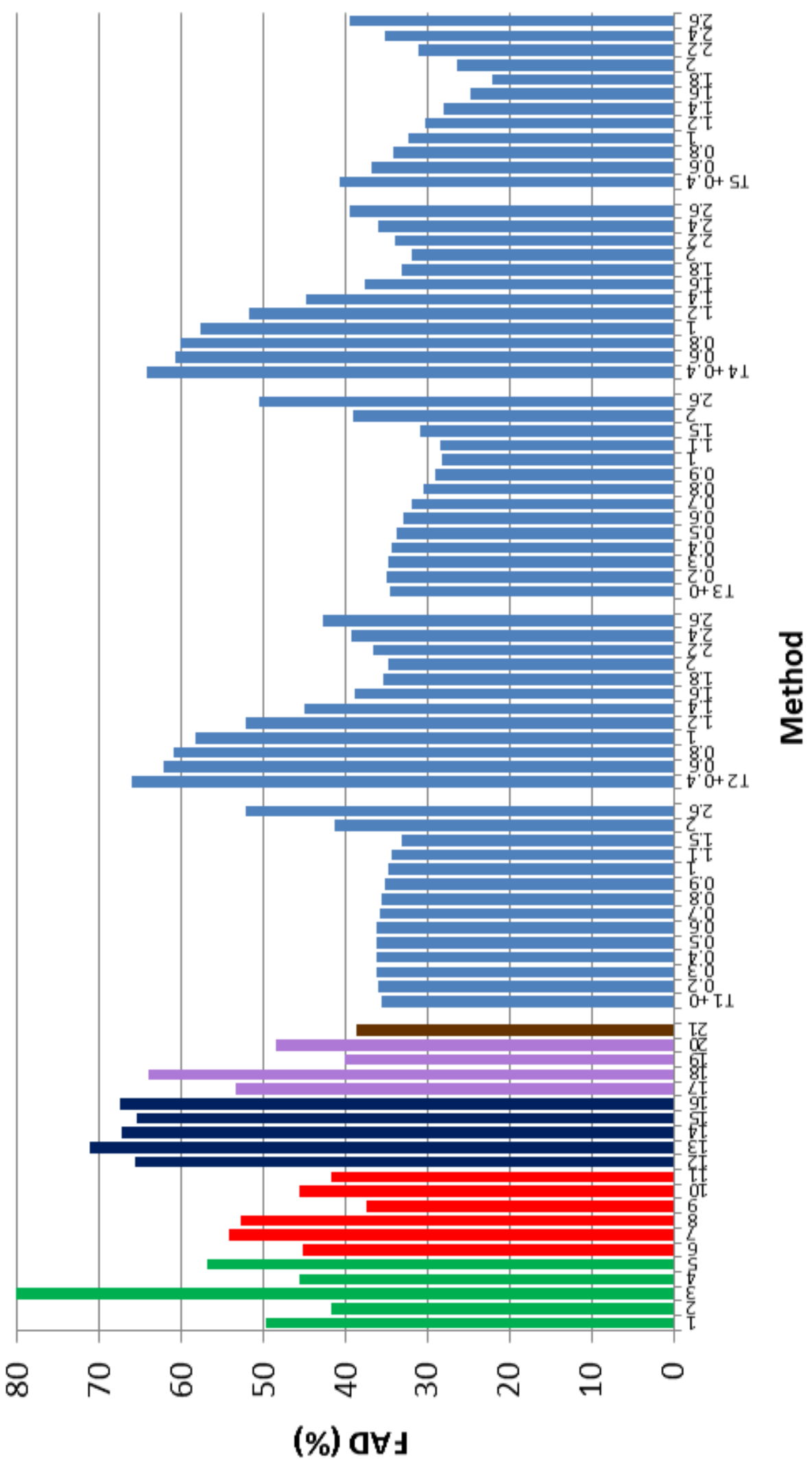

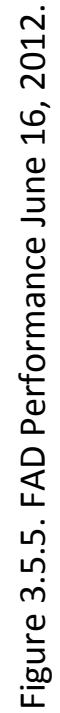




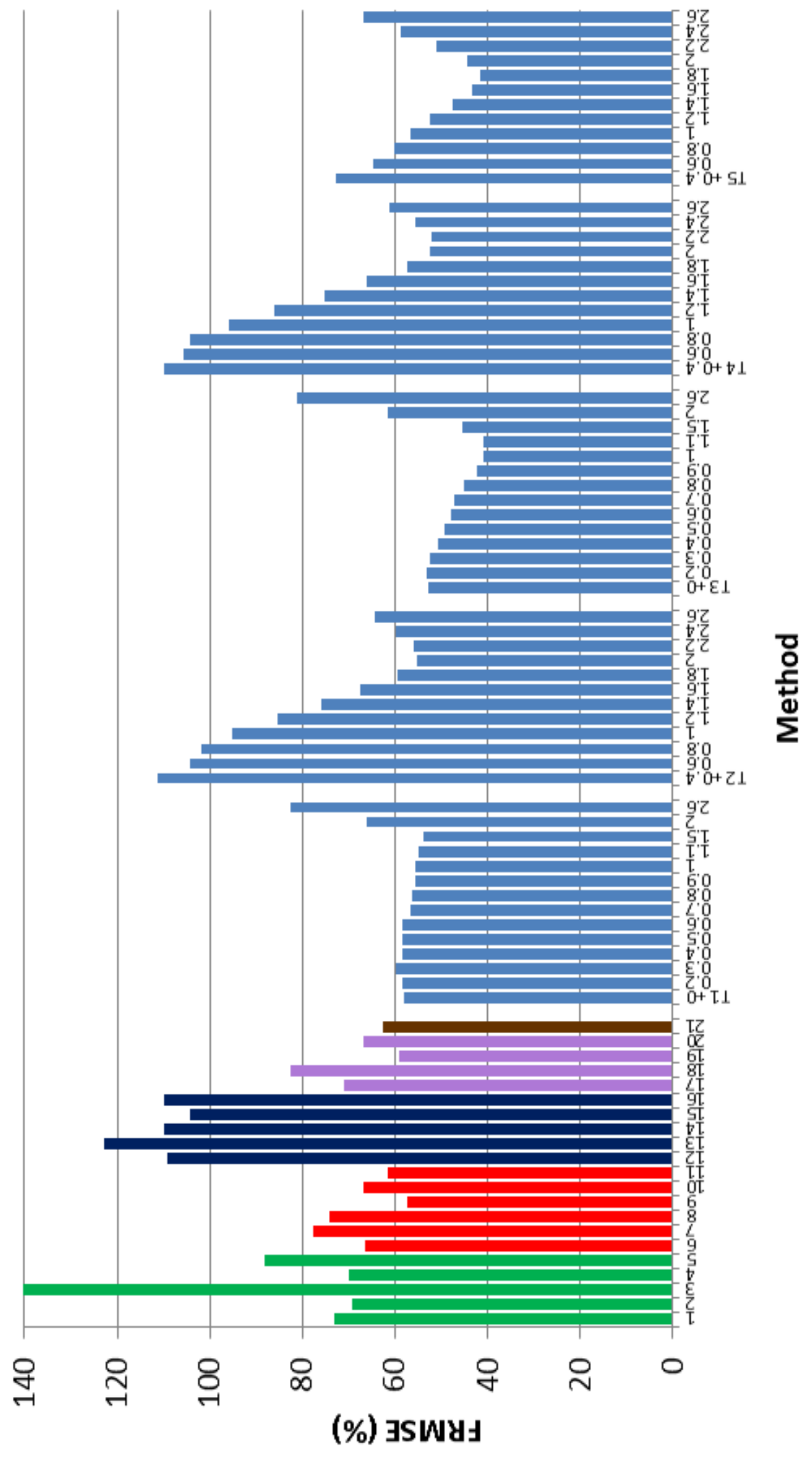

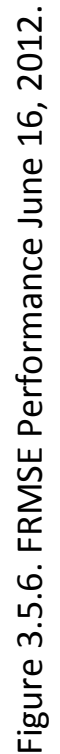




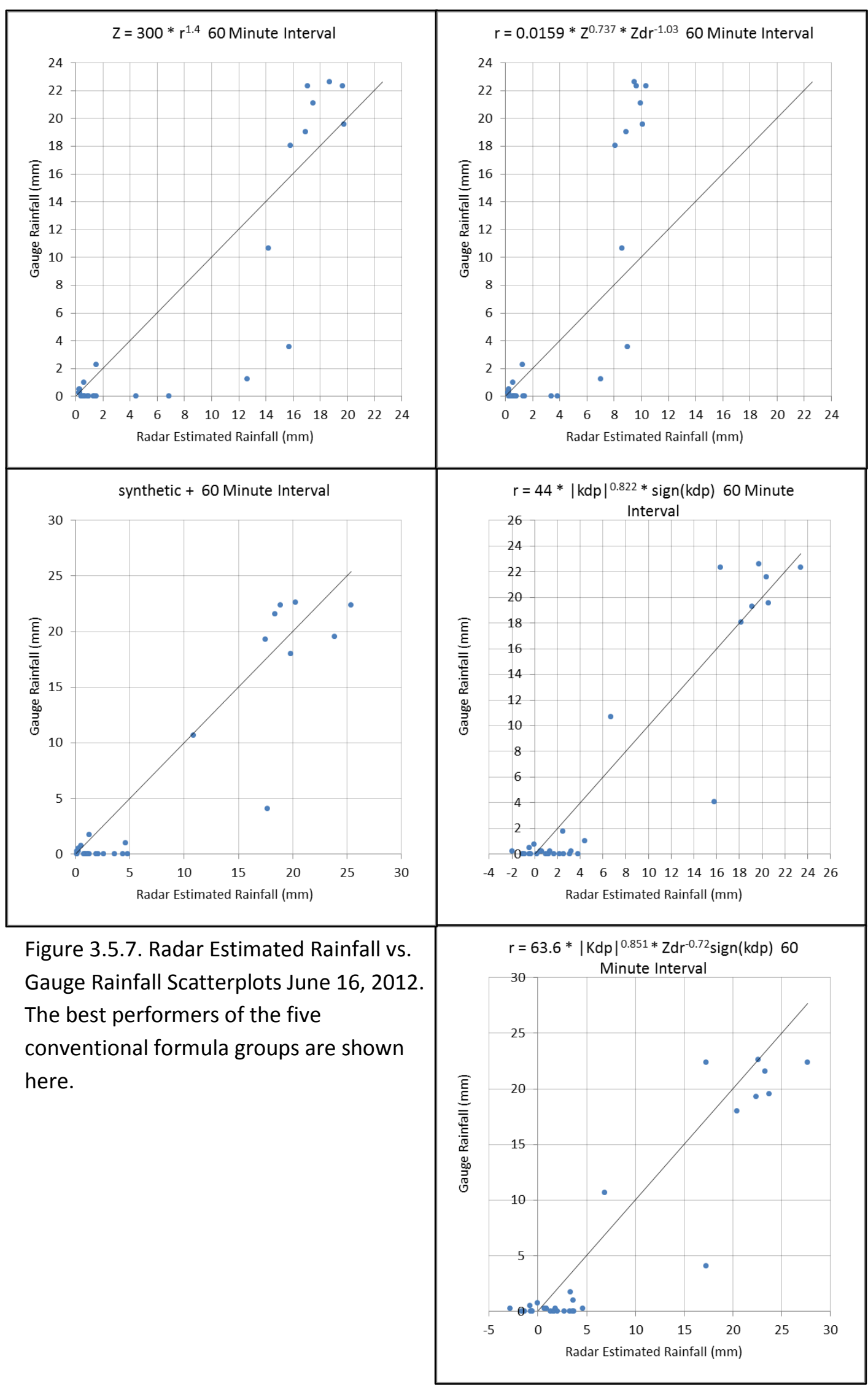



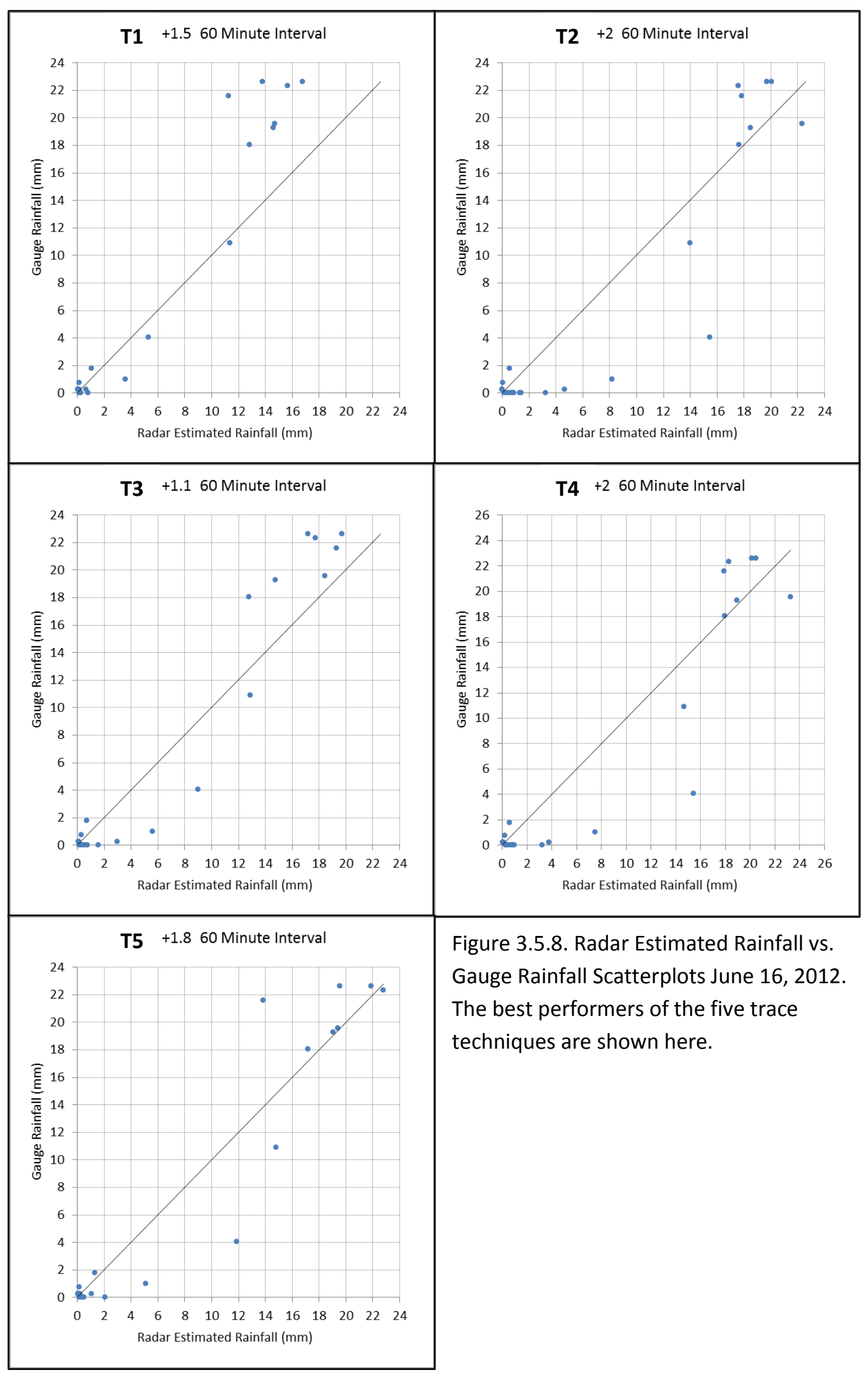

Figure 3.5.8. Radar Estimated Rainfall vs.

Gauge Rainfall Scatterplots June 16, 2012.

The best performers of the five trace techniques are shown here. 


\subsection{August 31, 2012}

On the $31^{\text {st }}$ of August the remnants of Hurricane Isaac passed through Central Missouri, helping to ease the severe drought conditions affecting the region. Figure 3.6.1 is an HPC surface analysis for 15 UTC of August 31, 2012. The precipitation on this day came in the form of continuous, heavy rainfall. Figure 3.6.2 shows humidity versus height in percentile format for 11 UTC through 22 UTC of August 31. Initially the atmosphere was relatively dry, but quickly became fully saturated. Figure 3.6 .3 is a hodograph for the $31^{\text {st }}$ of August. The hodograph shows an easterly wind at the surface turning more in a southeasterly direction higher up. These wind directions are expected considering Central Missouri's position on the northern side of the cyclone. Figure 3.6.4 is a four panel radar image from KLSX for 1537 UTC of August 31.

\subsubsection{Conventional Formulas}

Table 3.6.1 shows the performance of the conventional formulas for this event. The synthetic formula, (21), was the most effective of the conventional formulas evaluated. The tropical Z-R relationship, (3), had its best showing, and the least negative bias out of all the conventional formulas. The $\mathrm{K}_{\mathrm{DP}}$-reliant formulas performed slightly worse overall, except for (21). Every conventional formula tested showed a significant negative bias for this event. 


\subsubsection{Tracing Techniques}

Table 3.6.2 shows the performance of the various trace techniques and their

permutations for this event. All five tracing techniques were most effective at $+Z_{D R}$ values of around 0.8 on this date. This optimal $+Z_{D R}$ value was anomalously low for $T 2$, T4, and T5.

The overall effectiveness of the optimal tracing techniques was superior to the conventional techniques for this event.

\subsubsection{Graphical Evaluation}

Figure 3.6.5 is a histogram showing the FAD performance of each evaluated technique for this event. Figure 3.6.6 is the equivalent histogram showing FRMSE performance.

Figure 3.6.7 shows a combination of scatterplots of radar-derived rainfall versus rain gauge rainfall for the conventional formulas. The techniques illustrated were the top performers in each category. Figure 3.6.8 is identical to 3.6.7, except it depicts the best performers of the trace techniques. 


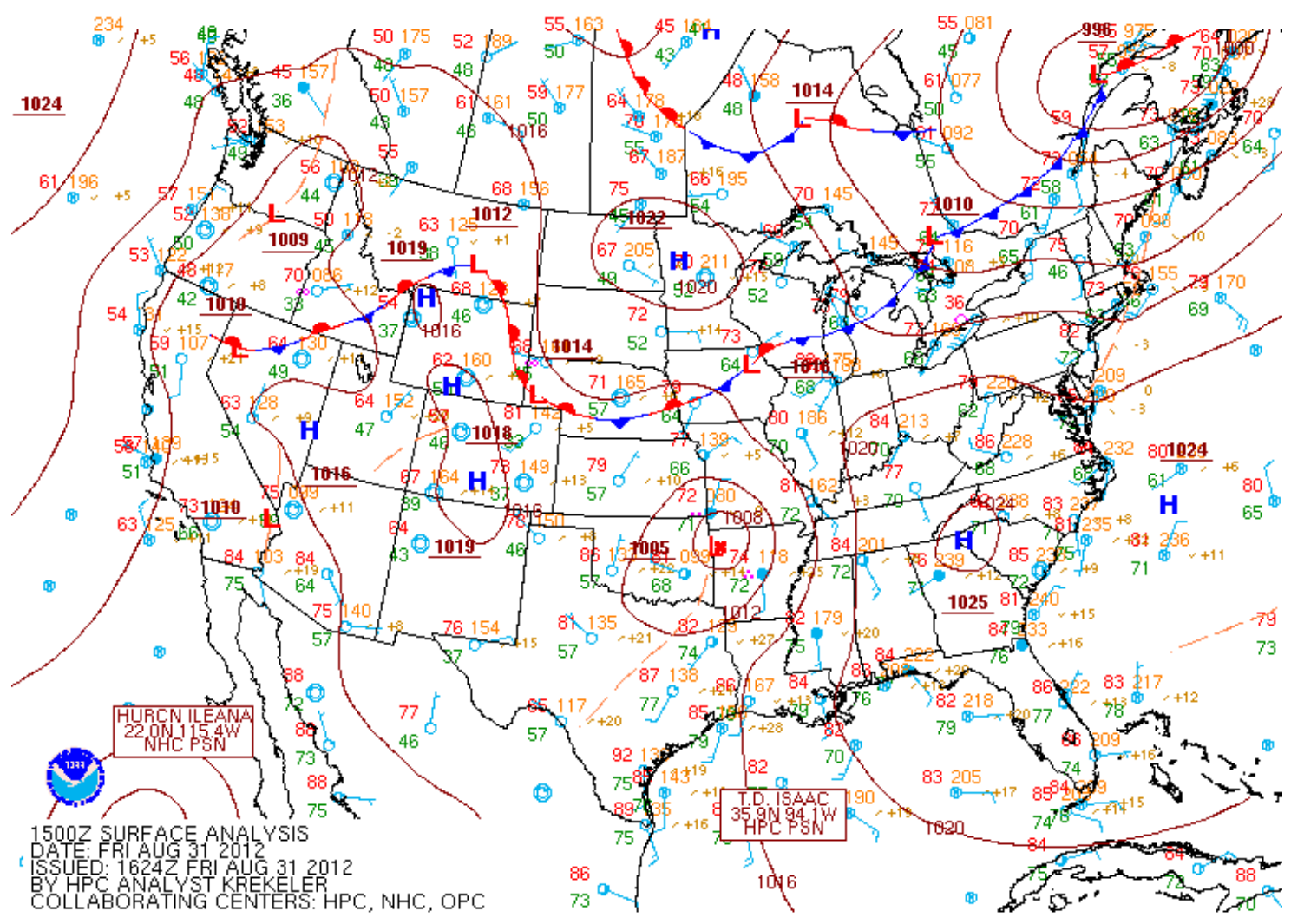

Figure 3.6.1. Surface Analysis 1500 UTC August 31, 2012.

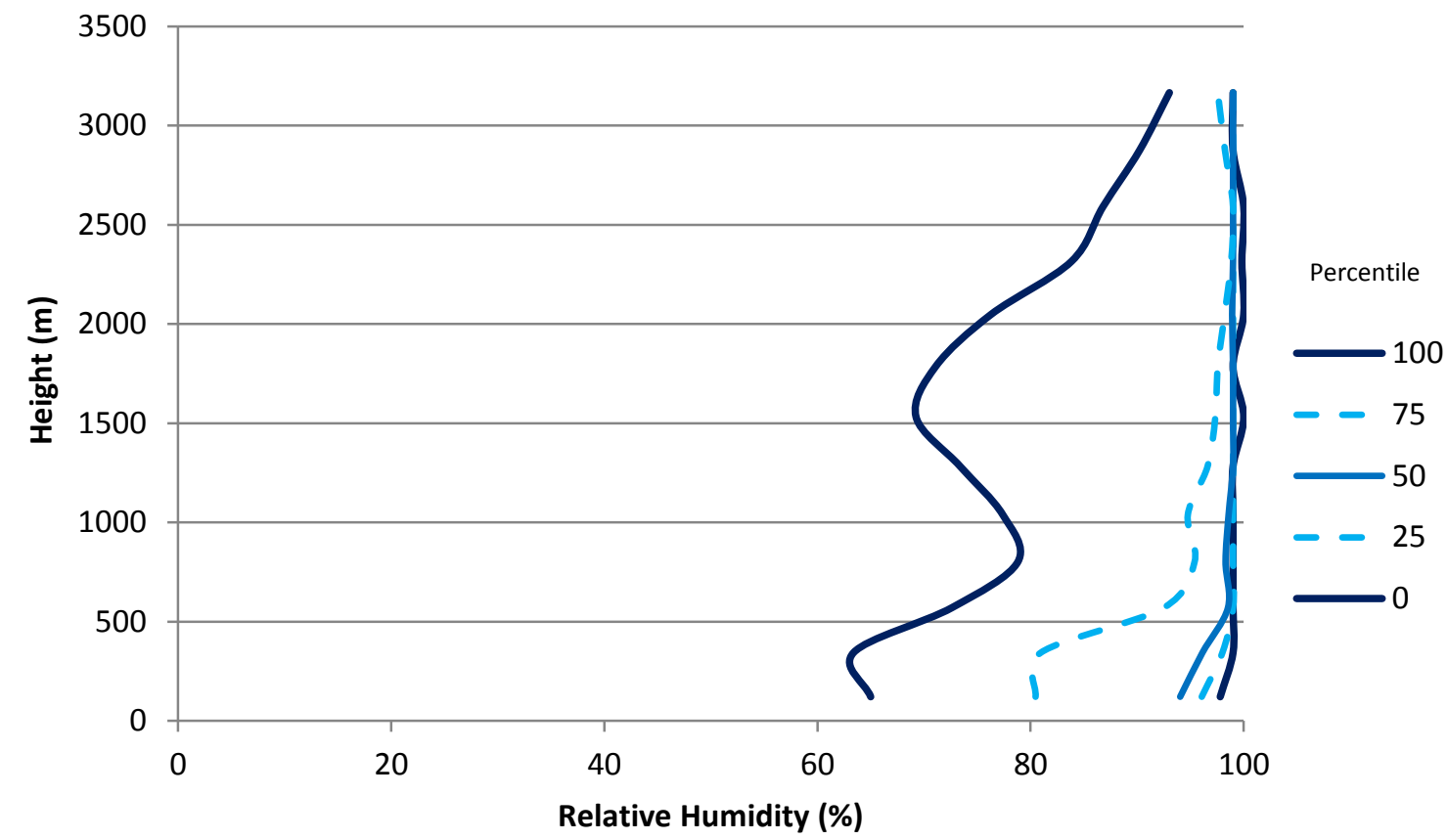

Figure 3.6.2. Relative Humidity Profile August 31, 2012. 


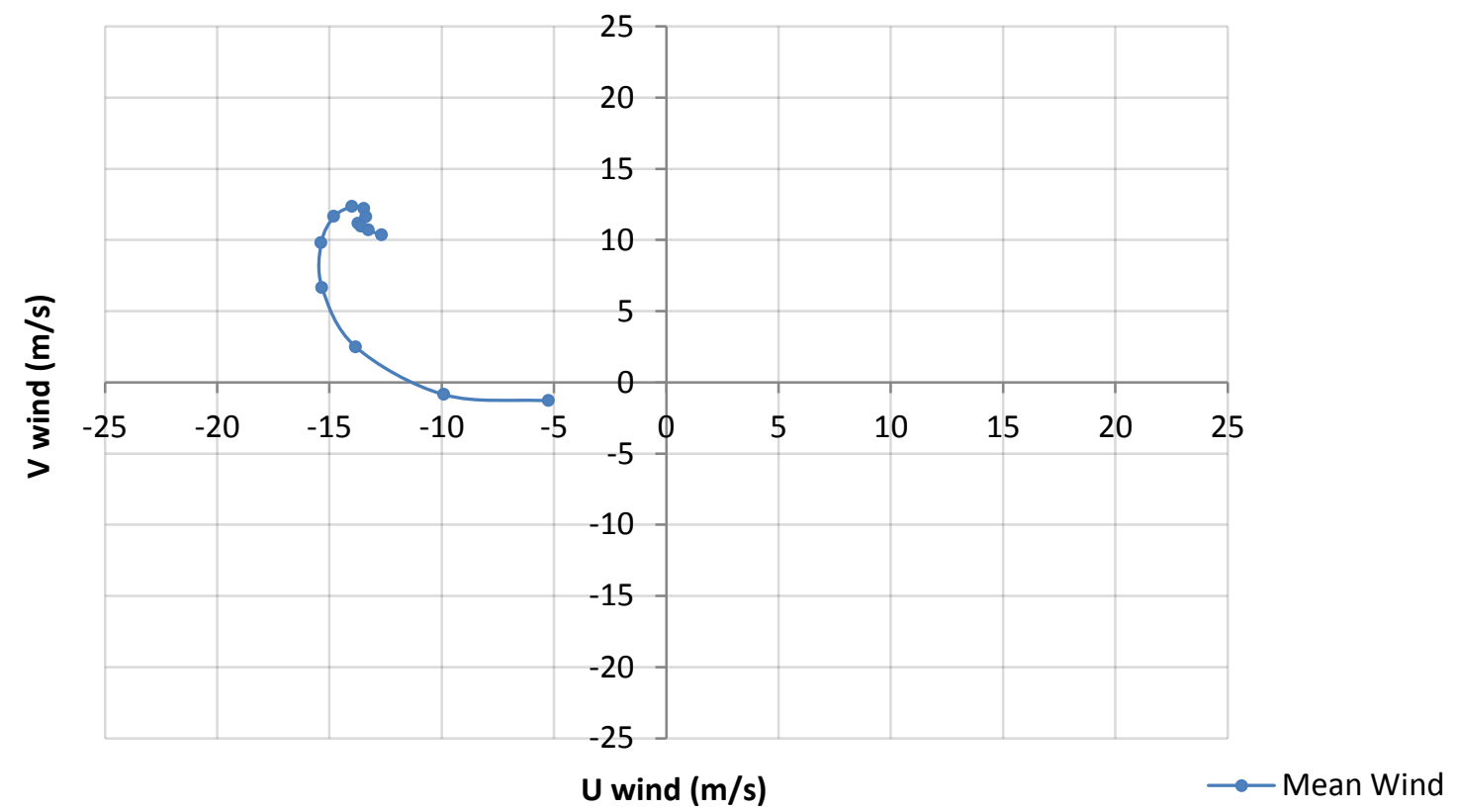

Figure 3.6.3. Hodograph August 31, 2012. 


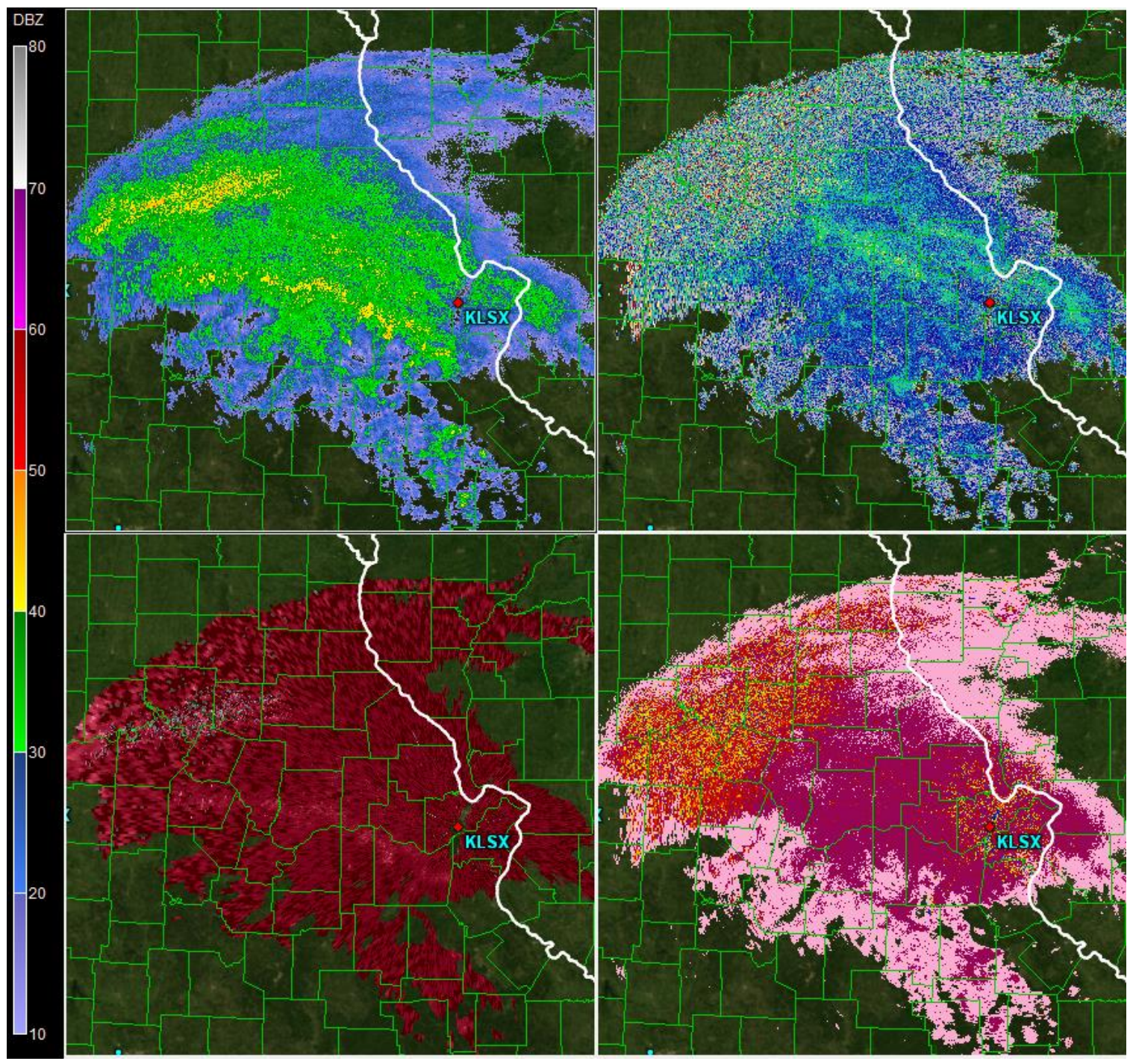

Figure 3.6.4. Four Panel Radar Image for 1537 UTC August 31, 2012. The top left is reflectivity $(Z)$, top right is differential reflectivity $\left(Z_{D R}\right)$, bottom left is specific phase differential $\left(K_{D P}\right)$, and bottom right is correlation coefficient. The scale on the left corresponds to the top left image (Z). The elevation angle used here was $0.5^{\circ}$. 
Table 3.6.1 Conventional Techniques, August 31, 2012

\begin{tabular}{|c|c|c|c|c|c|}
\hline$Z=a R^{b}$ & $\#$ & $\mathrm{FB}(\%)$ & $\mathrm{FAD}(\%)$ & FRMSE(\%) & $\mathrm{FSD}(\%)$ \\
\hline 1 & 74 & -35.44 & 48.53 & 67.25 & 57.15 \\
\hline 2 & 74 & -40.04 & 50.08 & 69.41 & 56.70 \\
\hline 3 & 74 & -10.47 & 42.85 & 52.89 & 51.84 \\
\hline 4 & 74 & -42.82 & 51.63 & 72.03 & 57.92 \\
\hline 5 & 74 & -60.21 & 63.08 & 88.25 & 64.53 \\
\hline \multicolumn{6}{|c|}{$R\left(K_{D P}\right)=a\left|K_{D P}\right|^{b} \operatorname{sign}\left(K_{D P}\right)$} \\
\hline 6 & 72 & -46.45 & 64.12 & 82.94 & 68.71 \\
\hline 7 & 72 & -38.28 & 63.21 & 81.17 & 71.57 \\
\hline 8 & 72 & -31.01 & 63.87 & 80.37 & 74.14 \\
\hline 9 & 72 & -51.31 & 64.96 & 84.38 & 66.99 \\
\hline 10 & 72 & -43.40 & 63.48 & 81.82 & 69.36 \\
\hline 11 & 72 & -44.87 & 63.44 & 81.92 & 68.54 \\
\hline \multicolumn{6}{|c|}{$R\left(Z, Z_{D R}\right)=a Z^{b} Z_{d r}^{c}$} \\
\hline 12 & 74 & -25.40 & 38.94 & 52.75 & 46.23 \\
\hline 13 & 74 & -20.42 & 35.68 & 48.61 & 44.11 \\
\hline 14 & 74 & -39.96 & 48.82 & 67.44 & 54.33 \\
\hline 15 & 74 & -42.79 & 50.92 & 70.76 & 56.36 \\
\hline 16 & 74 & -41.87 & 50.06 & 69.35 & 55.28 \\
\hline \multicolumn{6}{|c|}{$R\left(K_{D P}, Z_{D R}\right)=a\left|K_{D P}\right|^{b} Z_{d r}^{c} \operatorname{sign}\left(K_{D P}\right)$} \\
\hline 17 & 73 & -32.97 & 72.71 & 90.24 & 84.00 \\
\hline 18 & 73 & -19.36 & 87.01 & 107.57 & 105.81 \\
\hline 19 & 72 & -47.31 & 65.87 & 84.72 & 70.28 \\
\hline 20 & 73 & -38.25 & 66.36 & 84.80 & 75.69 \\
\hline \multicolumn{6}{|l|}{ Synthetic } \\
\hline 21 & 73 & -17.89 & 36.42 & 46.99 & 43.45 \\
\hline
\end{tabular}


Table 3.6.2 Trace Techniques, August 31, 2012

\begin{tabular}{|c|c|c|c|c|c|c|}
\hline & $Z_{D R}+$ & $\#$ & $\mathrm{FB}(\%)$ & $\mathrm{FAD}(\%)$ & FRMSE(\%) & $\mathrm{FSD}(\%)$ \\
\hline \multirow[t]{14}{*}{ T1 } & 0 & 73 & 88.35 & 105.03 & 146.74 & 117.16 \\
\hline & 0.2 & 73 & 54.11 & 73.64 & 97.60 & 81.22 \\
\hline & 0.3 & 73 & 39.63 & 61.74 & 79.13 & 68.49 \\
\hline & 0.4 & 73 & 26.99 & 51.63 & 64.59 & 58.68 \\
\hline & 0.5 & 73 & 16.06 & 44.74 & 53.93 & 51.48 \\
\hline & 0.6 & 73 & 6.49 & 39.87 & 46.98 & 46.53 \\
\hline & 0.7 & 73 & -1.95 & 36.80 & 43.36 & 43.32 \\
\hline & 0.8 & 73 & -9.41 & 34.38 & 42.55 & 41.49 \\
\hline & 0.9 & 73 & -16.05 & 33.06 & 43.77 & 40.73 \\
\hline & 1 & 73 & -21.94 & 33.75 & 46.25 & 40.71 \\
\hline & 1.1 & 73 & -27.20 & 35.98 & 49.39 & 41.23 \\
\hline & 1.5 & 73 & -43.46 & 46.54 & 63.17 & 45.84 \\
\hline & 2 & 73 & -56.99 & 58.89 & 77.51 & 52.53 \\
\hline & 2.6 & 73 & -67.78 & 68.92 & 89.98 & 59.18 \\
\hline \multirow[t]{12}{*}{$\mathrm{T} 2$} & 0.4 & 73 & 33.33 & 53.68 & 67.04 & 58.17 \\
\hline & 0.6 & 73 & 10.06 & 39.86 & 47.45 & 46.37 \\
\hline & 0.8 & 73 & -7.54 & 35.06 & 43.01 & 42.34 \\
\hline & 1 & 73 & -20.91 & 36.82 & 47.21 & 42.32 \\
\hline & 1.2 & 73 & -31.34 & 41.23 & 54.17 & 44.19 \\
\hline & 1.4 & 73 & -39.69 & 46.25 & 61.35 & 46.78 \\
\hline & 1.6 & 73 & -46.53 & 51.10 & 67.95 & 49.52 \\
\hline & 1.8 & 73 & -52.23 & 55.57 & 73.83 & 52.19 \\
\hline & 2 & 73 & -57.05 & 59.69 & 79.03 & 54.68 \\
\hline & 2.2 & 73 & -61.20 & 63.44 & 83.61 & 56.97 \\
\hline & 2.4 & 73 & -64.79 & 66.69 & 87.67 & 59.06 \\
\hline & 2.6 & 73 & -67.93 & 69.54 & 91.27 & 60.96 \\
\hline \multirow[t]{14}{*}{ T3 } & 0 & 73 & 83.15 & 94.69 & 129.61 & 99.42 \\
\hline & 0.2 & 73 & 51.55 & 66.01 & 86.18 & 69.07 \\
\hline & 0.3 & 73 & 37.33 & 53.55 & 68.25 & 57.13 \\
\hline & 0.4 & 73 & 24.57 & 43.14 & 53.94 & 48.02 \\
\hline & 0.5 & 73 & 13.39 & 35.09 & 43.86 & 41.76 \\
\hline & 0.6 & 73 & 3.64 & 31.12 & 38.19 & 38.02 \\
\hline & 0.7 & 73 & -4.93 & 29.85 & 36.61 & 36.27 \\
\hline & 0.8 & 73 & -12.41 & 30.41 & 37.98 & 35.90 \\
\hline & 0.9 & 73 & -18.96 & 32.09 & 41.08 & 36.45 \\
\hline & 1 & 73 & -24.74 & 34.44 & 44.99 & 37.57 \\
\hline & 1.1 & 73 & -29.86 & 36.86 & 49.15 & 39.04 \\
\hline & 1.5 & 73 & -45.62 & 48.15 & 64.68 & 45.84 \\
\hline & 2 & 73 & -58.66 & 59.98 & 79.31 & 53.37 \\
\hline & 2.6 & 73 & -69.03 & 69.75 & 91.58 & 60.19 \\
\hline
\end{tabular}


Table 3.6.2 Trace Techniques, August 31, 2012 (Continued)

\begin{tabular}{|c|c|c|c|c|c|c|}
\hline & $Z_{D R}+$ & $\#$ & $\mathrm{FB}(\%)$ & FAD(\%) & FRMSE(\%) & $\mathrm{FSD}(\%)$ \\
\hline \multirow[t]{12}{*}{ T4 } & 0.4 & 73 & 33.32 & 50.28 & 63.35 & 53.88 \\
\hline & 0.6 & 73 & 10.06 & 34.49 & 42.86 & 41.66 \\
\hline & 0.8 & 73 & -7.54 & 31.83 & 38.63 & 37.89 \\
\hline & 1 & 73 & -20.91 & 34.48 & 43.81 & 38.50 \\
\hline & 1.2 & 73 & -31.34 & 38.89 & 51.61 & 41.01 \\
\hline & 1.4 & 73 & -39.69 & 44.05 & 59.37 & 44.15 \\
\hline & 1.6 & 73 & -46.53 & 49.33 & 66.37 & 47.33 \\
\hline & 1.8 & 73 & -52.23 & 54.29 & 72.54 & 50.34 \\
\hline & 2 & 73 & -57.06 & 58.64 & 77.94 & 53.10 \\
\hline & 2.2 & 73 & -61.20 & 62.50 & 82.69 & 55.60 \\
\hline & 2.4 & 73 & -64.79 & 65.85 & 86.87 & 57.87 \\
\hline & 2.6 & 73 & -67.94 & 68.80 & 90.58 & 59.91 \\
\hline \multirow[t]{12}{*}{ T5 } & 0.4 & 73 & 35.76 & 58.62 & 73.68 & 64.42 \\
\hline & 0.6 & 73 & 12.97 & 44.03 & 51.97 & 50.32 \\
\hline & 0.8 & 73 & -4.44 & 37.04 & 44.07 & 43.84 \\
\hline & 1 & 73 & -18.02 & 34.44 & 45.73 & 42.03 \\
\hline & 1.2 & 73 & -28.72 & 38.05 & 51.51 & 42.75 \\
\hline & 1.4 & 73 & -37.34 & 42.92 & 58.27 & 44.74 \\
\hline & 1.6 & 73 & -44.41 & 47.93 & 64.82 & 47.22 \\
\hline & 1.8 & 73 & -50.33 & 52.97 & 70.81 & 49.81 \\
\hline & 2 & 73 & -55.34 & 57.57 & 76.17 & 52.33 \\
\hline & 2.2 & 73 & -59.65 & 61.53 & 80.94 & 54.71 \\
\hline & 2.4 & 73 & -63.38 & 64.97 & 85.18 & 56.91 \\
\hline & 2.6 & 73 & -66.66 & 68.00 & 88.97 & 58.93 \\
\hline
\end{tabular}




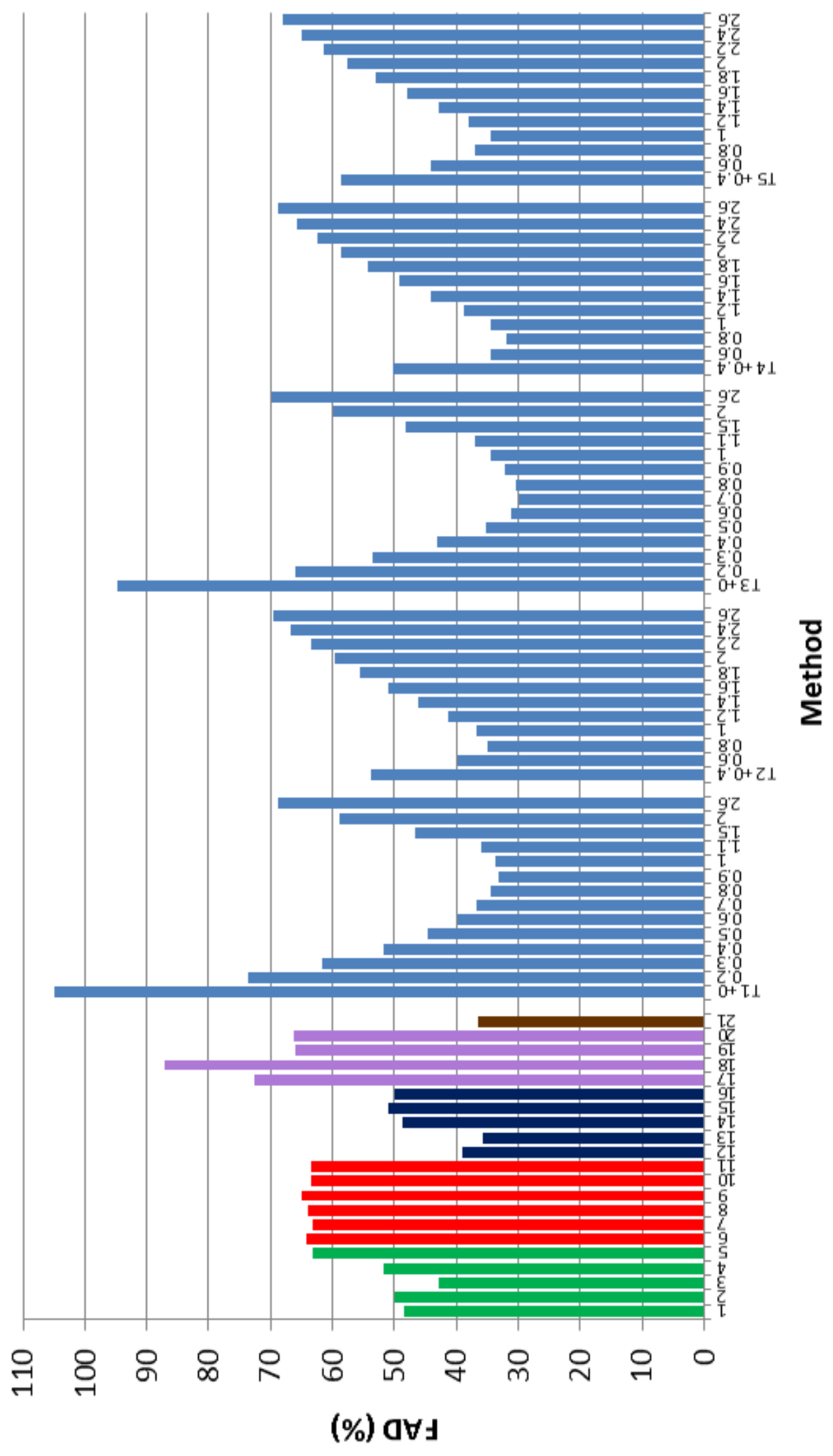

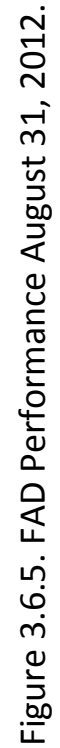




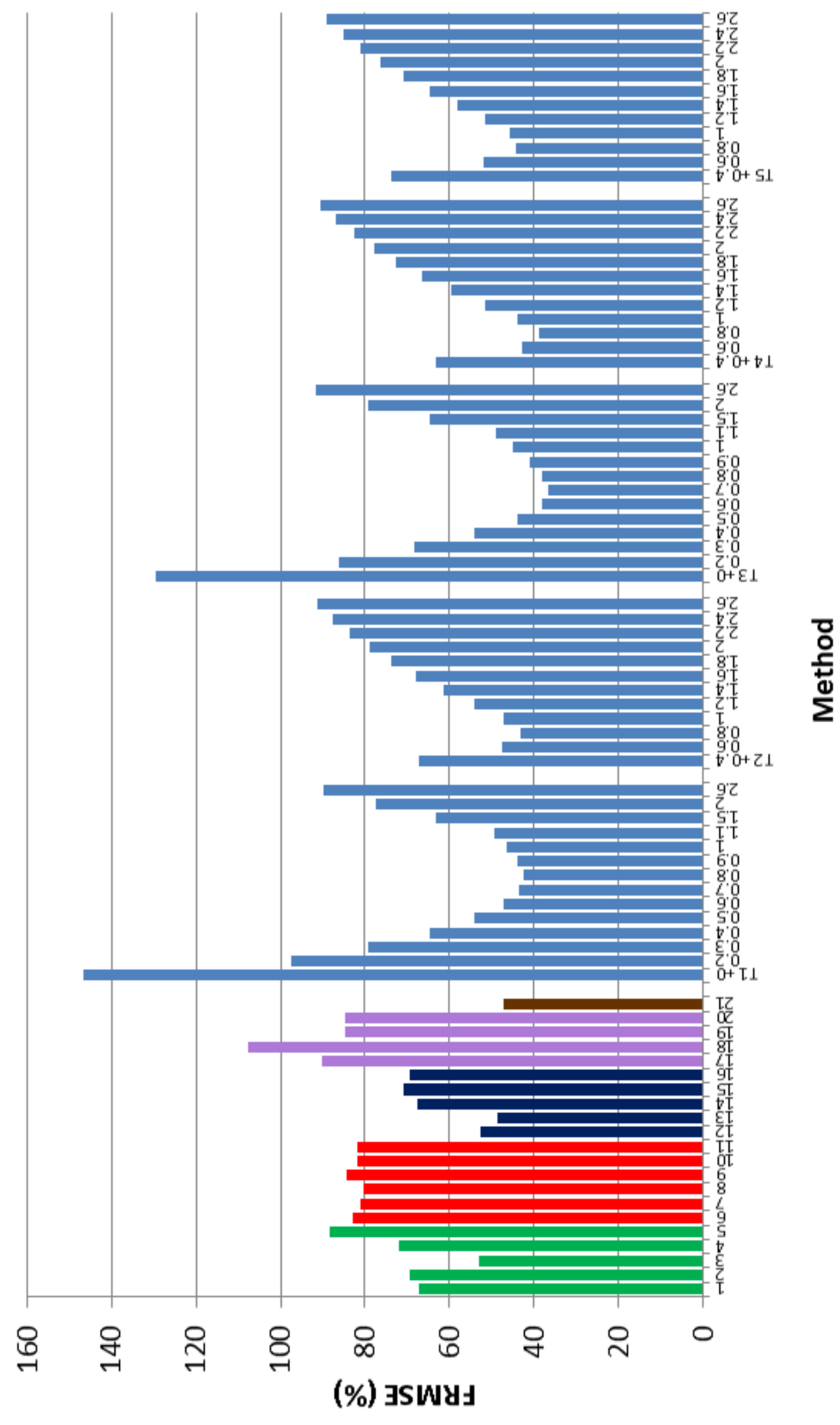

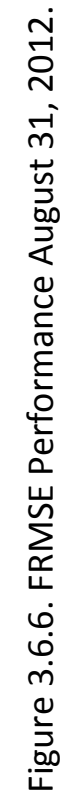




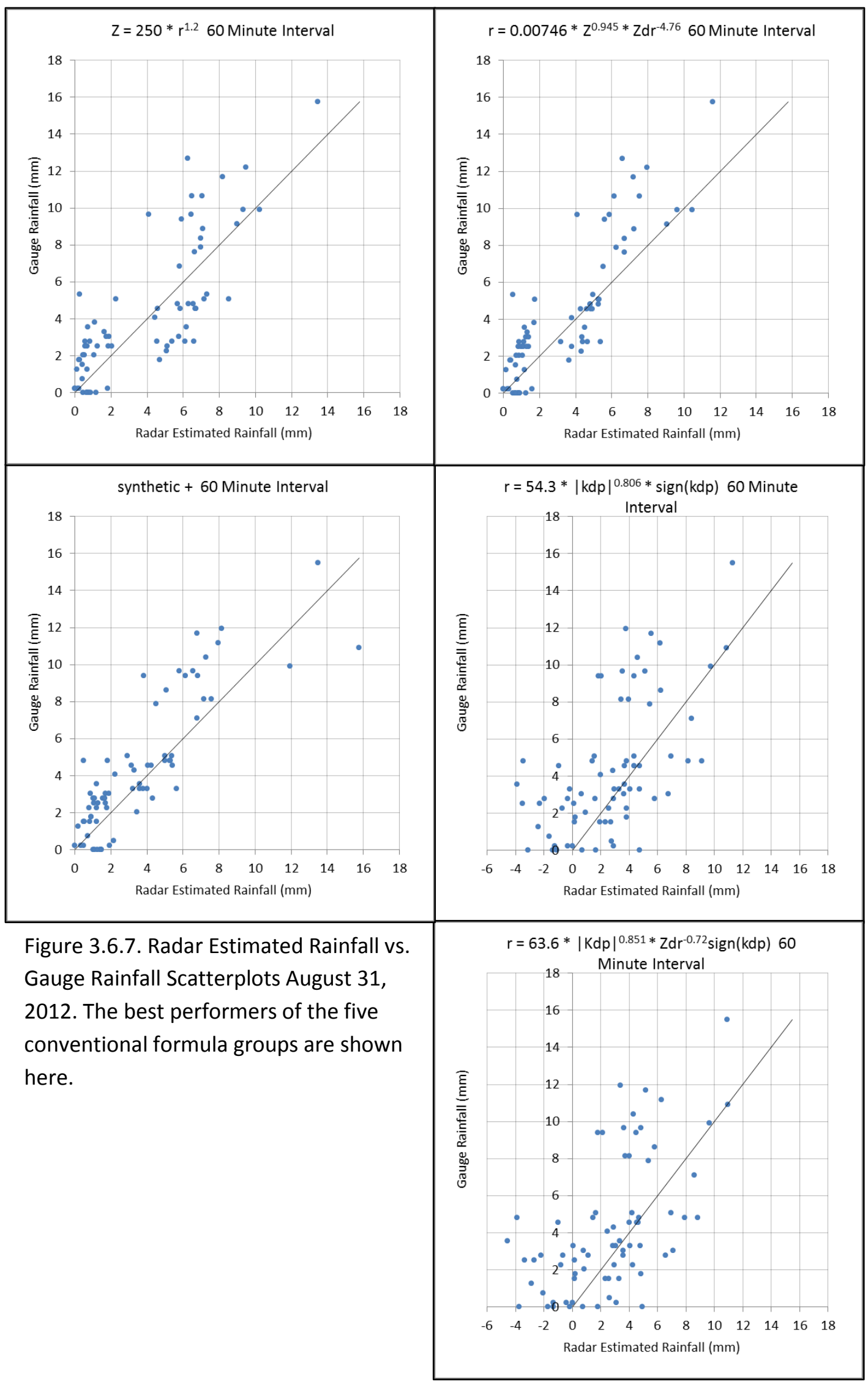



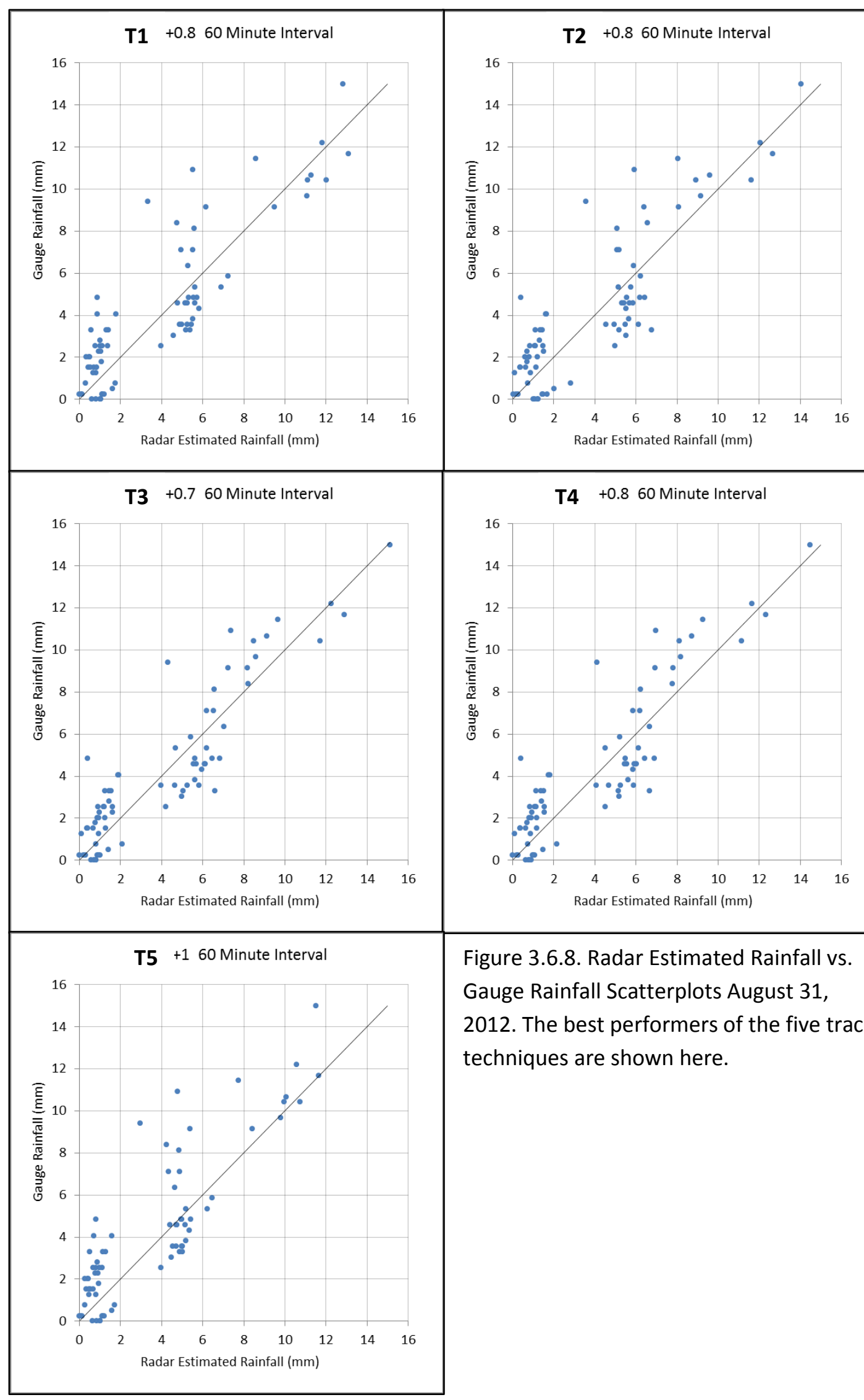

Figure 3.6.8. Radar Estimated Rainfall vs. Gauge Rainfall Scatterplots August 31, 2012. The best performers of the five trace techniques are shown here. 


\subsection{September 7, 2012}

The afternoon of September 7 saw a severe hailstorm pass through Columbia, Missouri. Figure 3.7.1 is an HPC surface analysis for 21 UTC of September 7, 2012. The storms were associated with a cold front and low pressure center moving from the northwest to southeast. Reflectivity values for the storm passing through Columbia reached between 65 and $70 \mathrm{dBZ}$, a clear indication of hail. Figure 3.7.2 shows humidity versus height in percentile format for 19 UTC of September 7 through 01 UTC of September 8. On this afternoon, the atmosphere made a rapid transition from dry to moist. Figure 3.7.3 is a hodograph for the $31^{\text {st }}$ of August. The hodograph shows a northwesterly wind at the surface turning northerly at 925 millibars, and transitioning to westerly higher up. Figure 3.7.4 is a four panel radar image from KLSX for 2107 UTC of September 7.

\subsubsection{Conventional Formulas}

Table 3.7.1 shows the performance of the conventional formulas for this event. The presence of large hail contributed to extremely poor performance for all of the conventional formulas. The best performer was the synthetic formula (21). Overall the $K_{D P}$-reliant formulas outperformed the other formulas for this event. Three outliers were present, $(3,12$, and 13$)$. Biases for the conventional techniques were all very positive for this date. 


\subsubsection{Tracing Techniques}

Table 3.7.2 shows the performance of the various trace techniques and their permutations for this event. The optimal T1 and T3 techniques performed significantly better than the other tracing techniques and all of the conventional formulas. The optimal $Z_{D R}$ adjustment for all five tracing techniques was again roughly +0.8 . The performance of the other tracing techniques was roughly on par with the conventional formulas. Every trace technique permutation tested exhibited a positive bias for this event.

\subsubsection{Graphical Evaluation}

Figure 3.7.5 is a histogram showing the FAD performance of each evaluated technique for this event. Figure 3.7.6 is the equivalent histogram showing FRMSE performance.

Figure 3.7.7 shows a combination of scatterplots of radar-derived rainfall versus rain gauge rainfall for the conventional formulas. The techniques illustrated were the top performers in each category. Figure 3.7.8 is identical to 3.7.7, except it depicts the best performers of the trace techniques. 


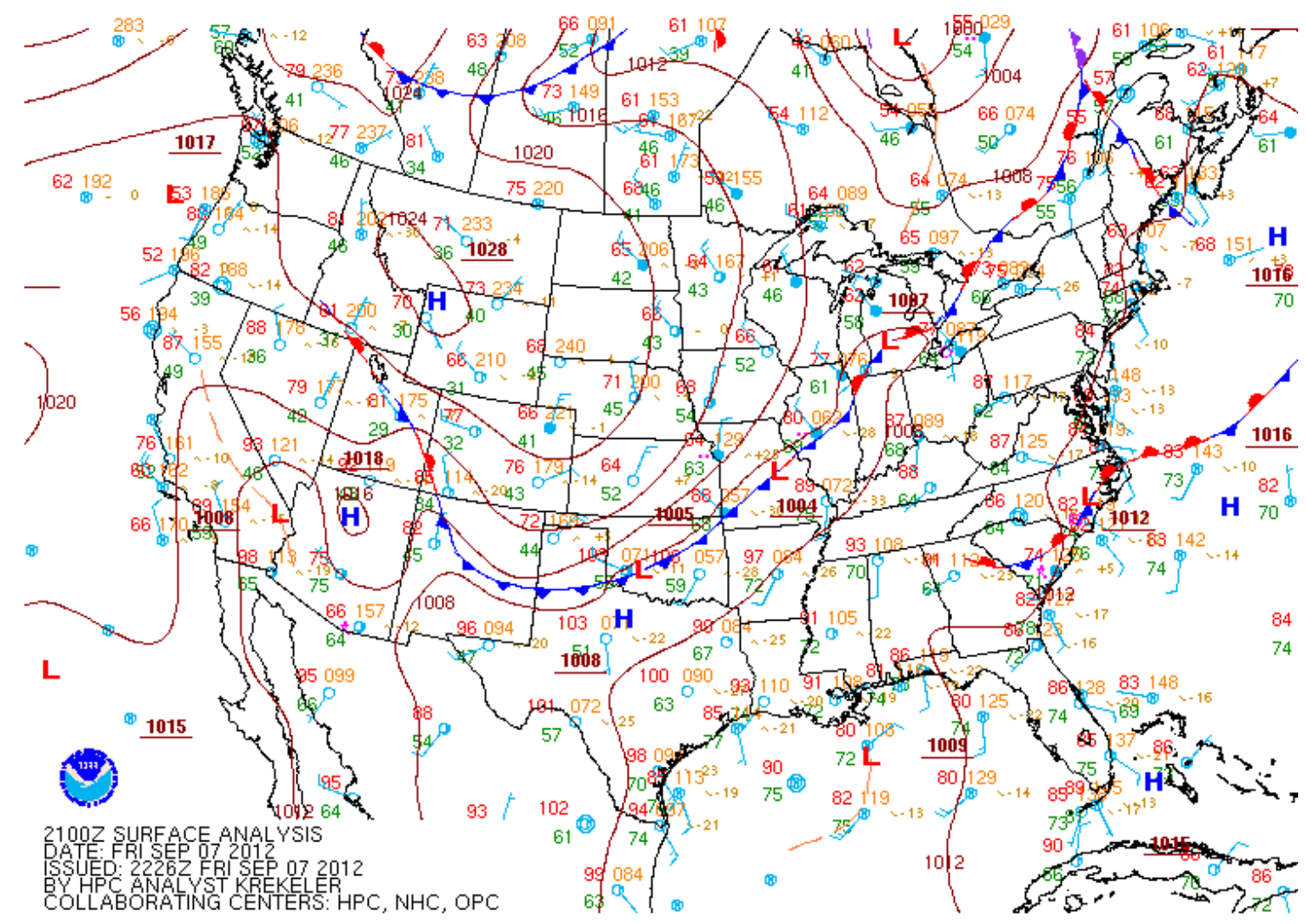

Figure 3.7.1. Surface Analysis 2100 UTC September 7, 2012.

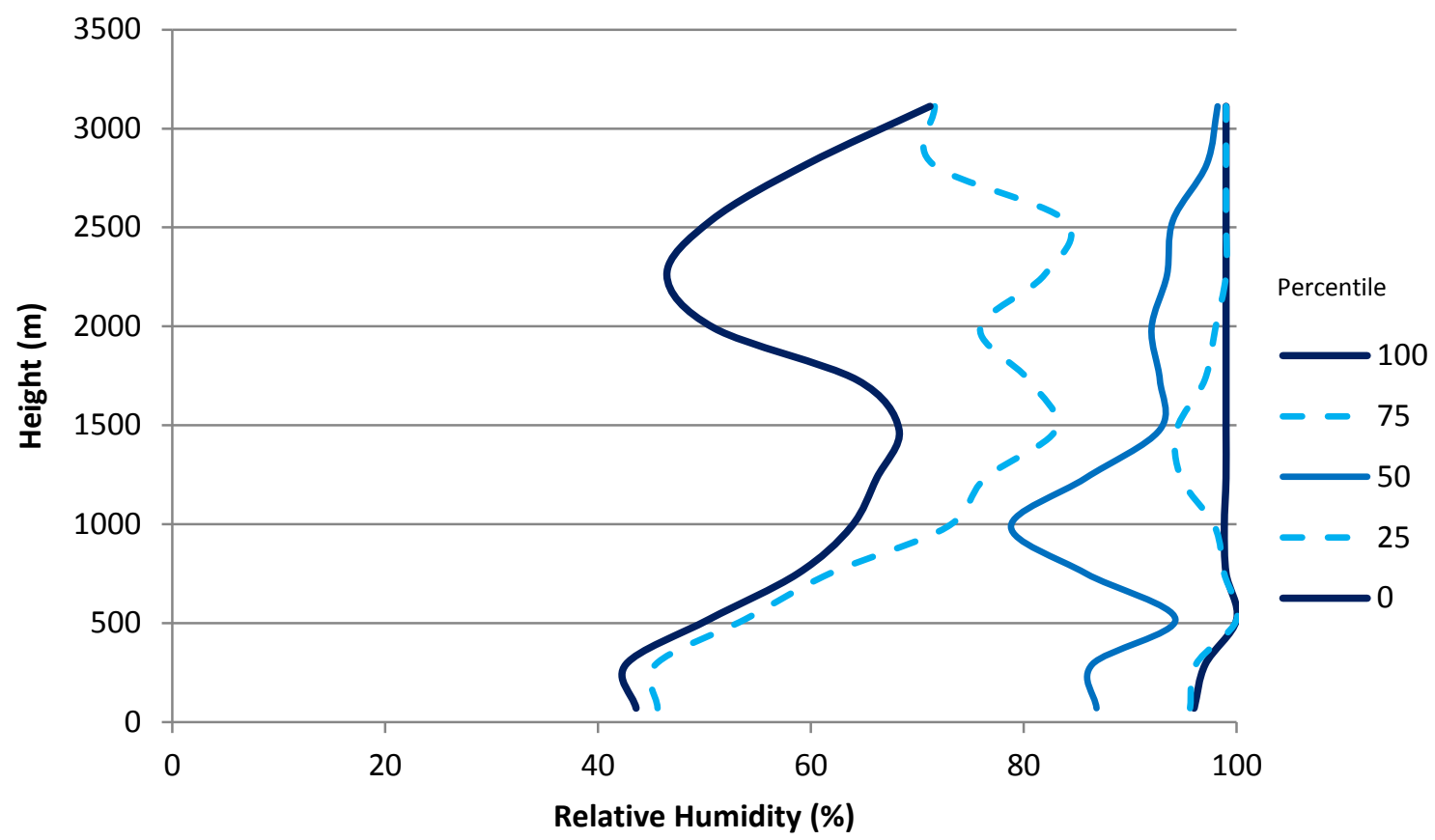

Figure 3.7.2. Relative Humidity Profile September 7, 2012. 


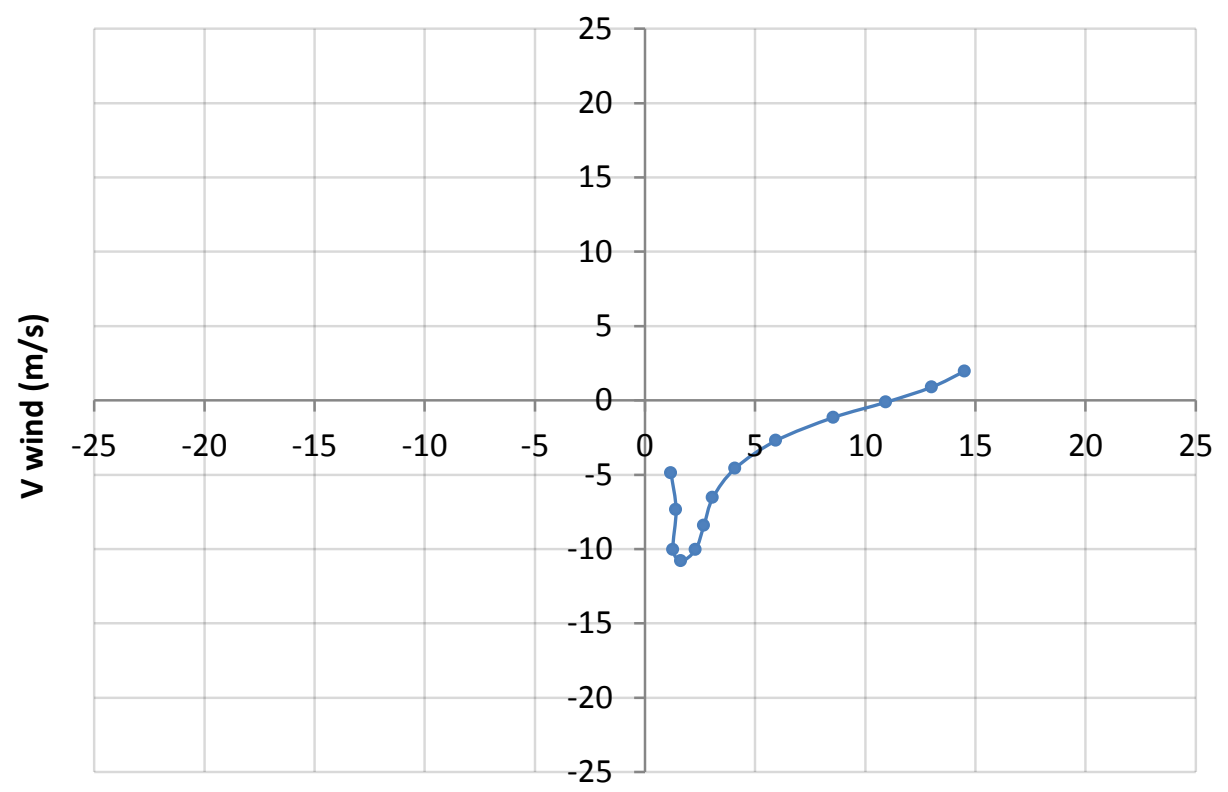

$\mathrm{U}$ wind $(\mathrm{m} / \mathrm{s}) \quad \rightarrow$ Mean Wind

Figure 3.7.3. Hodograph September 7, 2012. 


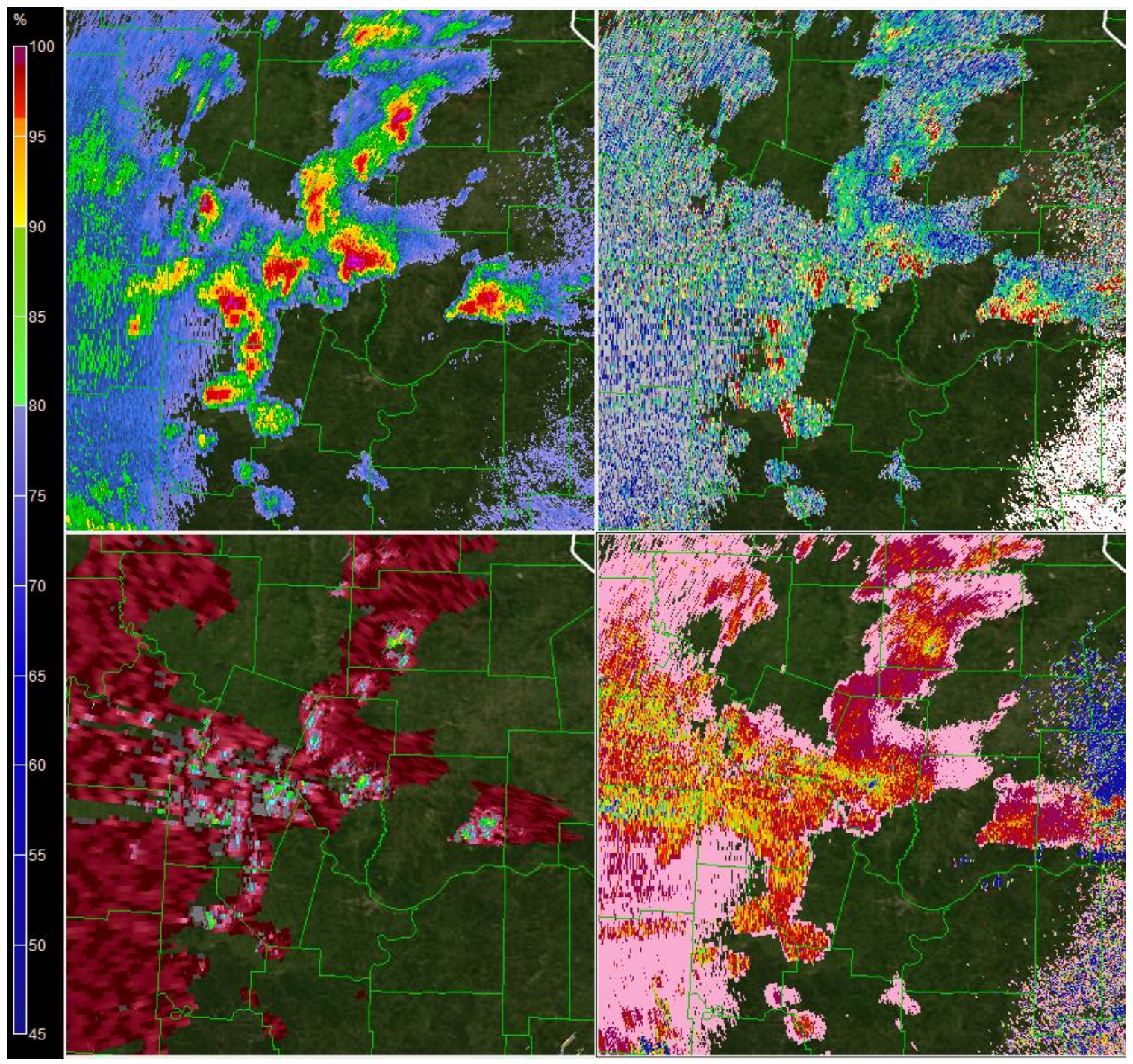

Figure 3.7.4. Four Panel Radar Image for 2107 UTC September 7, 2012. The top left is reflectivity $(Z)$, top right is differential reflectivity $\left(Z_{D R}\right)$, bottom left is specific phase differential $\left(K_{D P}\right)$, and bottom right is correlation coefficient. The scale on the left corresponds to the bottom right image (correlation coefficient). The elevation angle used here was $0.5^{\circ}$. 
Table 3.7.1 Conventional Techniques, September 7, 2012

\begin{tabular}{|c|c|c|c|c|c|}
\hline$Z=a R^{b}$ & \# & $\mathrm{FB}(\%)$ & $\mathrm{FAD}(\%)$ & FRMSE(\%) & FSD(\%) \\
\hline 1 & 35 & 17.31 & 64.49 & 113.48 & 112.15 \\
\hline 2 & 35 & 34.72 & 76.21 & 142.59 & 138.30 \\
\hline 3 & 35 & 179.27 & 192.10 & 363.50 & 316.22 \\
\hline 4 & 35 & 22.23 & 70.13 & 128.58 & 126.64 \\
\hline 5 & 35 & -11.73 & 68.78 & 113.94 & 113.33 \\
\hline \multicolumn{6}{|c|}{$R\left(K_{D P}\right)=a\left|K_{D P}\right|^{b} \operatorname{sign}\left(K_{D P}\right)$} \\
\hline 6 & 35 & 83.47 & 120.77 & 164.77 & 142.06 \\
\hline 7 & 36 & 100.26 & 136.54 & 186.94 & 157.78 \\
\hline 8 & 36 & 99.35 & 135.72 & 184.83 & 155.85 \\
\hline 9 & 35 & 61.07 & 99.72 & 137.83 & 123.56 \\
\hline 10 & 35 & 84.92 & 122.12 & 165.67 & 142.25 \\
\hline 11 & 35 & 75.51 & 113.29 & 154.13 & 134.37 \\
\hline \multicolumn{6}{|c|}{$R\left(Z, Z_{D R}\right)=a Z^{b} Z_{d r}{ }^{c}$} \\
\hline 12 & 35 & -30.37 & 50.93 & 89.34 & 84.03 \\
\hline 13 & 35 & -36.61 & 55.61 & 95.83 & 88.56 \\
\hline 14 & 35 & -33.42 & 53.11 & 93.70 & 87.54 \\
\hline 15 & 35 & -29.70 & 54.13 & 92.90 & 88.03 \\
\hline 16 & 35 & -33.99 & 53.68 & 94.48 & 88.15 \\
\hline \multicolumn{6}{|c|}{$R\left(K_{D P}, Z_{D R}\right)=a\left|K_{D P}\right|^{b} Z_{d r}^{c} \operatorname{sign}\left(K_{D P}\right)$} \\
\hline 17 & 35 & 142.70 & 184.44 & 253.66 & 209.71 \\
\hline 18 & 36 & 199.21 & 244.75 & 348.22 & 285.61 \\
\hline 19 & 35 & 79.31 & 119.80 & 162.59 & 141.93 \\
\hline 20 & 35 & 108.78 & 148.61 & 200.21 & 168.08 \\
\hline \multicolumn{6}{|l|}{ Synthetic } \\
\hline 21 & 34 & 45.75 & 79.41 & 121.47 & 112.53 \\
\hline
\end{tabular}


Table 3.7.2 Trace Techniques, September 7, 2012

\begin{tabular}{|c|c|c|c|c|c|c|}
\hline & $Z_{D R}+$ & $\#$ & $\mathrm{FB}(\%)$ & FAD(\%) & FRMSE(\%) & $\mathrm{FSD}(\%)$ \\
\hline \multirow[t]{14}{*}{ T1 } & 0 & 36 & 43.35 & 62.76 & 92.82 & 82.08 \\
\hline & 0.2 & 36 & 34.08 & 53.56 & 78.07 & 70.24 \\
\hline & 0.3 & 36 & 30.03 & 49.73 & 72.60 & 66.11 \\
\hline & 0.4 & 36 & 26.70 & 46.38 & 68.79 & 63.40 \\
\hline & 0.5 & 36 & 23.99 & 44.13 & 66.16 & 61.66 \\
\hline & 0.6 & 36 & 21.38 & 41.81 & 63.67 & 59.97 \\
\hline & 0.7 & 33 & 19.03 & 39.55 & 59.77 & 56.66 \\
\hline & 0.8 & 32 & 17.58 & 38.69 & 59.12 & 56.45 \\
\hline & 0.9 & 32 & 16.80 & 38.67 & 60.62 & 58.25 \\
\hline & 1 & 32 & 16.61 & 39.39 & 63.13 & 60.91 \\
\hline & 1.1 & 32 & 16.82 & 41.60 & 66.77 & 64.62 \\
\hline & 1.5 & 32 & 21.79 & 58.16 & 92.80 & 90.20 \\
\hline & 2 & 31 & 25.69 & 73.91 & 121.15 & 118.40 \\
\hline & 2.6 & 31 & 18.48 & 78.03 & 132.78 & 131.49 \\
\hline \multirow[t]{12}{*}{$\mathrm{T} 2$} & 0.4 & 36 & 67.86 & 80.61 & 125.68 & 105.79 \\
\hline & 0.6 & 36 & 56.49 & 73.40 & 118.53 & 104.20 \\
\hline & 0.8 & 35 & 47.82 & 70.27 & 113.62 & 103.07 \\
\hline & 1 & 33 & 42.82 & 70.58 & 113.86 & 105.50 \\
\hline & 1.2 & 32 & 40.46 & 72.87 & 121.12 & 114.17 \\
\hline & 1.4 & 32 & 40.75 & 78.07 & 136.40 & 130.17 \\
\hline & 1.6 & 32 & 42.95 & 84.76 & 157.11 & 151.12 \\
\hline & 1.8 & 32 & 45.87 & 91.90 & 178.50 & 172.51 \\
\hline & 2 & 32 & 45.67 & 97.38 & 188.82 & 183.21 \\
\hline & 2.2 & 31 & 40.12 & 97.26 & 184.66 & 180.25 \\
\hline & 2.4 & 31 & 34.61 & 97.87 & 183.84 & 180.56 \\
\hline & 2.6 & 31 & 30.30 & 99.35 & 186.22 & 183.74 \\
\hline \multirow[t]{14}{*}{ T3 } & 0 & 36 & 36.72 & 64.66 & 95.61 & 88.28 \\
\hline & 0.2 & 36 & 28.83 & 56.87 & 86.73 & 81.80 \\
\hline & 0.3 & 36 & 25.53 & 53.69 & 83.86 & 79.88 \\
\hline & 0.4 & 36 & 22.68 & 51.10 & 81.85 & 78.65 \\
\hline & 0.5 & 36 & 19.93 & 49.21 & 80.12 & 77.60 \\
\hline & 0.6 & 36 & 16.84 & 47.18 & 77.52 & 75.66 \\
\hline & 0.7 & 36 & 14.18 & 46.05 & 75.59 & 74.24 \\
\hline & 0.8 & 36 & 12.17 & 45.71 & 74.75 & 73.75 \\
\hline & 0.9 & 36 & 10.78 & 45.63 & 74.94 & 74.16 \\
\hline & 1 & 36 & 9.82 & 45.75 & 76.14 & 75.50 \\
\hline & 1.1 & 34 & 8.85 & 46.17 & 76.01 & 75.49 \\
\hline & 1.5 & 32 & 9.46 & 52.69 & 90.70 & 90.21 \\
\hline & 2 & 32 & 12.30 & 67.23 & 117.52 & 116.87 \\
\hline & 2.6 & 32 & 1.30 & 70.53 & 122.59 & 122.58 \\
\hline
\end{tabular}


Table 3.7.2 Trace Techniques, September 7, 2012 (Continued)

\begin{tabular}{|c|c|c|c|c|c|c|}
\hline & $Z_{D R}+$ & $\#$ & $\mathrm{FB}(\%)$ & $\mathrm{FAD}(\%)$ & FRMSE(\%) & $\mathrm{FSD}(\%)$ \\
\hline \multirow[t]{12}{*}{ T4 } & 0.4 & 36 & 67.77 & 84.08 & 125.92 & 106.13 \\
\hline & 0.6 & 36 & 56.38 & 73.82 & 116.31 & 101.73 \\
\hline & 0.8 & 36 & 47.79 & 67.65 & 110.31 & 99.42 \\
\hline & 1 & 36 & 42.95 & 67.14 & 111.18 & 102.55 \\
\hline & 1.2 & 36 & 40.60 & 69.08 & 118.07 & 110.87 \\
\hline & 1.4 & 35 & 40.69 & 72.85 & 129.13 & 122.55 \\
\hline & 1.6 & 33 & 42.59 & 78.43 & 143.07 & 136.59 \\
\hline & 1.8 & 32 & 45.37 & 85.29 & 159.95 & 153.39 \\
\hline & 2 & 32 & 45.16 & 90.26 & 169.13 & 162.98 \\
\hline & 2.2 & 32 & 39.73 & 89.90 & 168.37 & 163.62 \\
\hline & 2.4 & 32 & 34.23 & 88.93 & 168.13 & 164.61 \\
\hline & 2.6 & 32 & 29.90 & 88.71 & 170.89 & 168.25 \\
\hline \multirow[t]{12}{*}{ T5 } & 0.4 & 36 & 70.80 & 76.88 & 117.43 & 93.69 \\
\hline & 0.6 & 36 & 61.03 & 68.65 & 106.46 & 87.23 \\
\hline & 0.8 & 36 & 54.90 & 65.00 & 103.57 & 87.82 \\
\hline & 1 & 34 & 52.44 & 65.41 & 105.81 & 91.90 \\
\hline & 1.2 & 32 & 52.93 & 69.87 & 114.07 & 101.05 \\
\hline & 1.4 & 32 & 56.49 & 78.05 & 132.23 & 119.55 \\
\hline & 1.6 & 32 & 59.95 & 85.39 & 151.63 & 139.27 \\
\hline & 1.8 & 32 & 63.18 & 93.44 & 169.96 & 157.78 \\
\hline & 2 & 31 & 63.97 & 98.83 & 179.51 & 167.72 \\
\hline & 2.2 & 31 & 60.38 & 99.80 & 183.22 & 172.99 \\
\hline & 2.4 & 31 & 57.60 & 100.78 & 188.80 & 179.80 \\
\hline & 2.6 & 31 & 54.83 & 101.06 & 193.16 & 185.21 \\
\hline
\end{tabular}




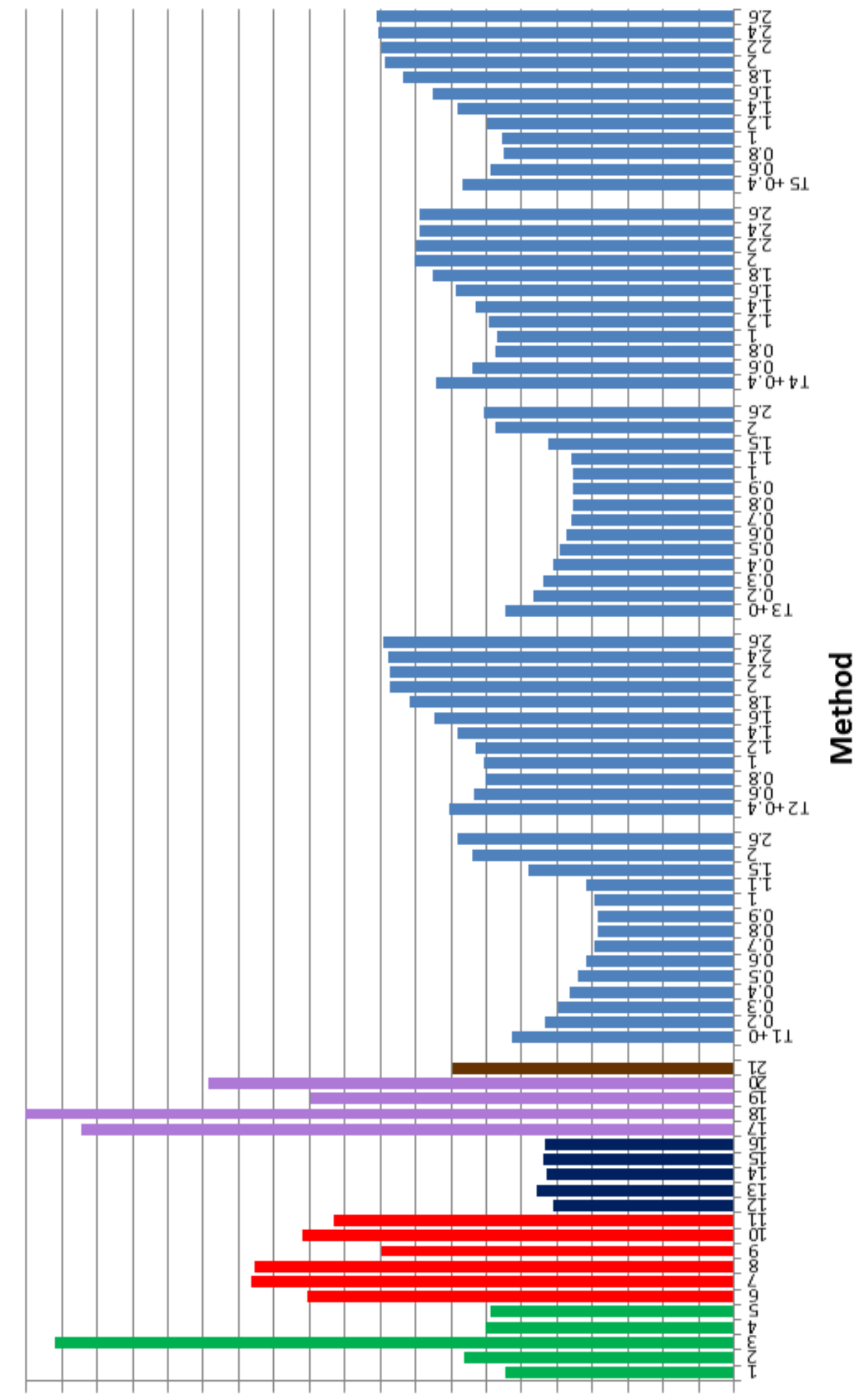

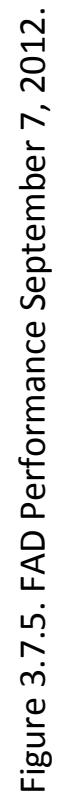




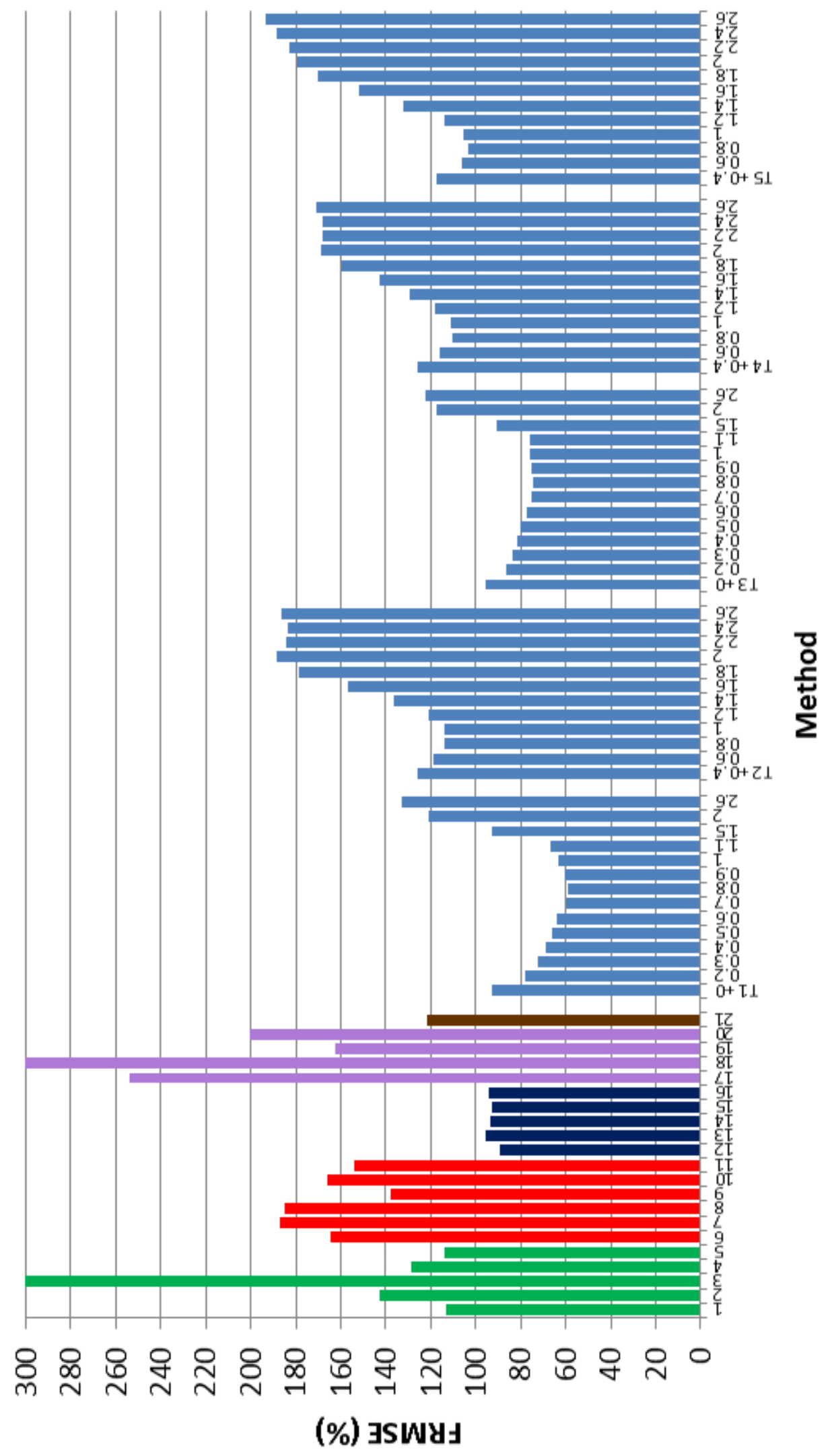

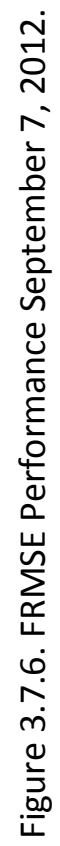




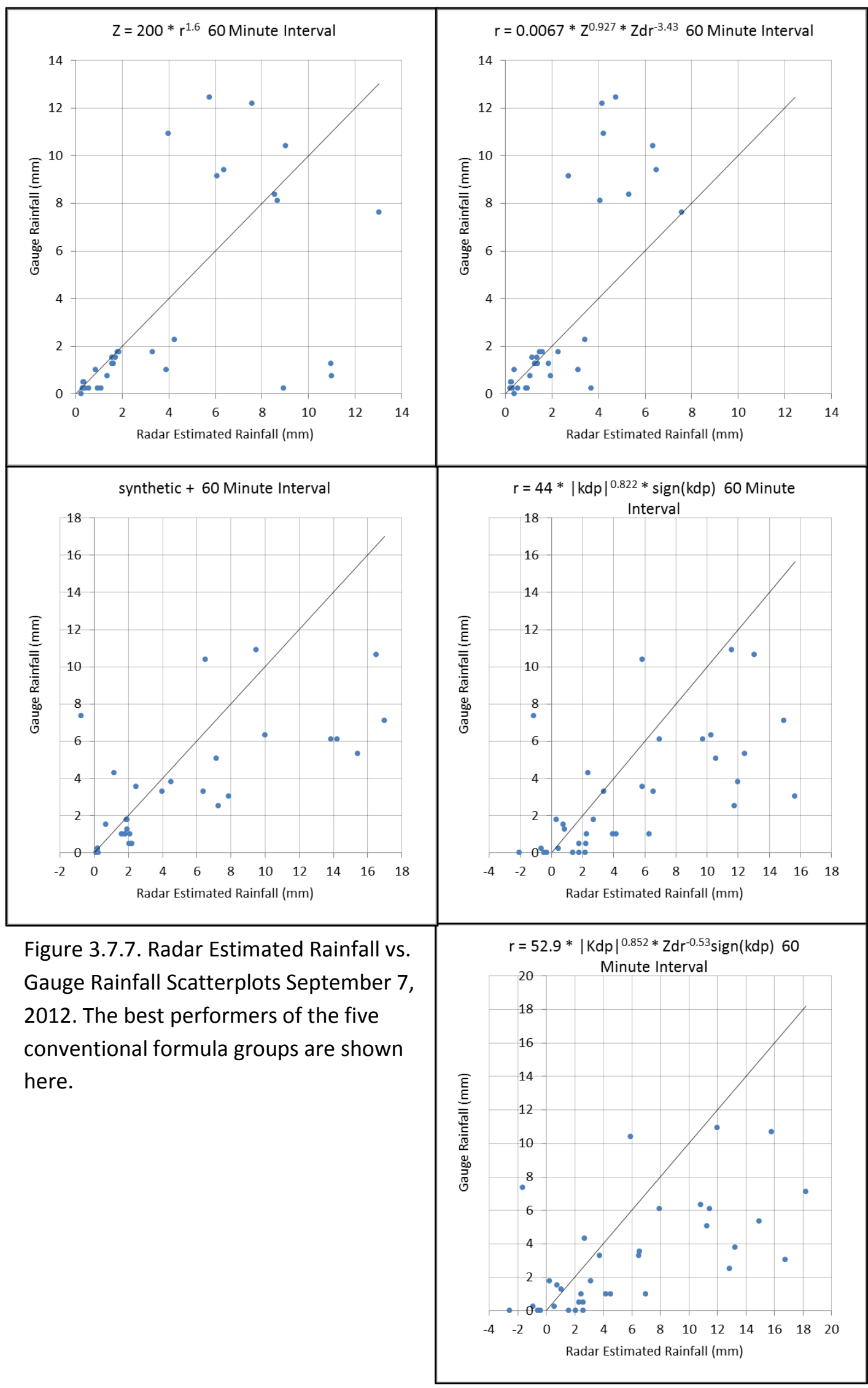



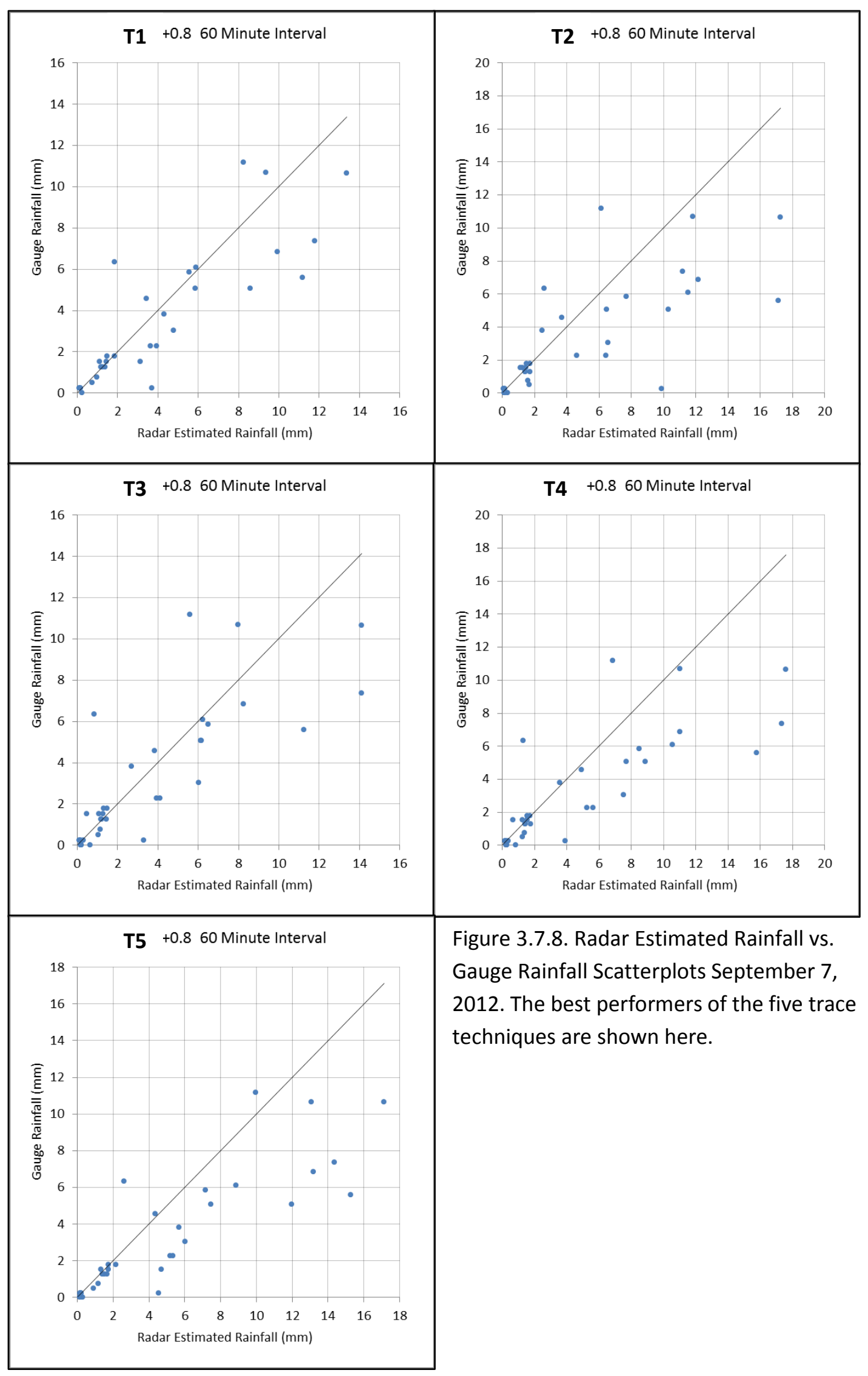

Figure 3.7.8. Radar Estimated Rainfall vs. Gauge Rainfall Scatterplots September 7, 2012. The best performers of the five trace techniques are shown here. 


\subsection{October 13, 2012}

The rainfall event on October 13 was associated with two centers of low pressure to the northwest of the region. On the $13^{\text {th }}$ of October several thunderstorm complexes moved through Central Missouri. Figure 3.8.1 is an HPC surface analysis for 03 UTC of October 14,2012 . Figure 3.8.2 shows humidity versus height in percentile format for 18 UTC of October 13 through 06 UTC of October 14. The atmosphere was very humid throughout this event. Figure 3.8 .3 is a hodograph for the $13^{\text {th }}$ of October. The hodograph shows a southerly wind at the surface turning southwesterly higher up.

\subsubsection{Conventional Formulas}

Table 3.8.1 shows the performance of the conventional formulas for this event. (1), (4), (14), (15), and (16) performed the best out of the conventional formulas. The tropical Z$\mathrm{R}$ formula, (3), performed poorly again. All $\mathrm{K}_{\mathrm{DP}}$-reliant formulas also performed very poorly on this date. Biases for the conventional formulas were nearly all positive for this date.

\subsubsection{Tracing Techniques}

Table 3.8.2 shows the performance of the various trace techniques and their permutations for this event. The optimal tracing techniques as a whole performed slightly better than the (non- $K_{D P}$ reliant) conventional formulas. The optimal $Z_{D R}$ adjustment for all five tracing techniques was roughly +2 , although this is less precise for $\mathrm{T} 1$ and $\mathrm{T} 3$ due to +1.8 and $+2.2 \mathrm{Z}_{\mathrm{DR}}$ adjustments not being tested. This was an anomalously high optimal $Z_{D R}$ adjustment for $\mathrm{T} 1$ and $\mathrm{T} 3$. This may have been caused by 
inaccurate humidity data within the high resolution model. Figure 3.8.2 indicated a very saturated environment, which would reduce expected evaporation.

\subsubsection{Graphical Evaluation}

Figure 3.8.4 is a histogram showing the FAD performance of each evaluated technique for this event. Figure 3.8.5 is the equivalent histogram showing FRMSE performance.

Figure 3.8.6 shows a combination of scatterplots of radar-derived rainfall versus rain gauge rainfall for the conventional formulas. The techniques illustrated were the top performers in each category. Figure 3.8.7 is identical to 3.8.6, except it depicts the best performers of the trace techniques. 


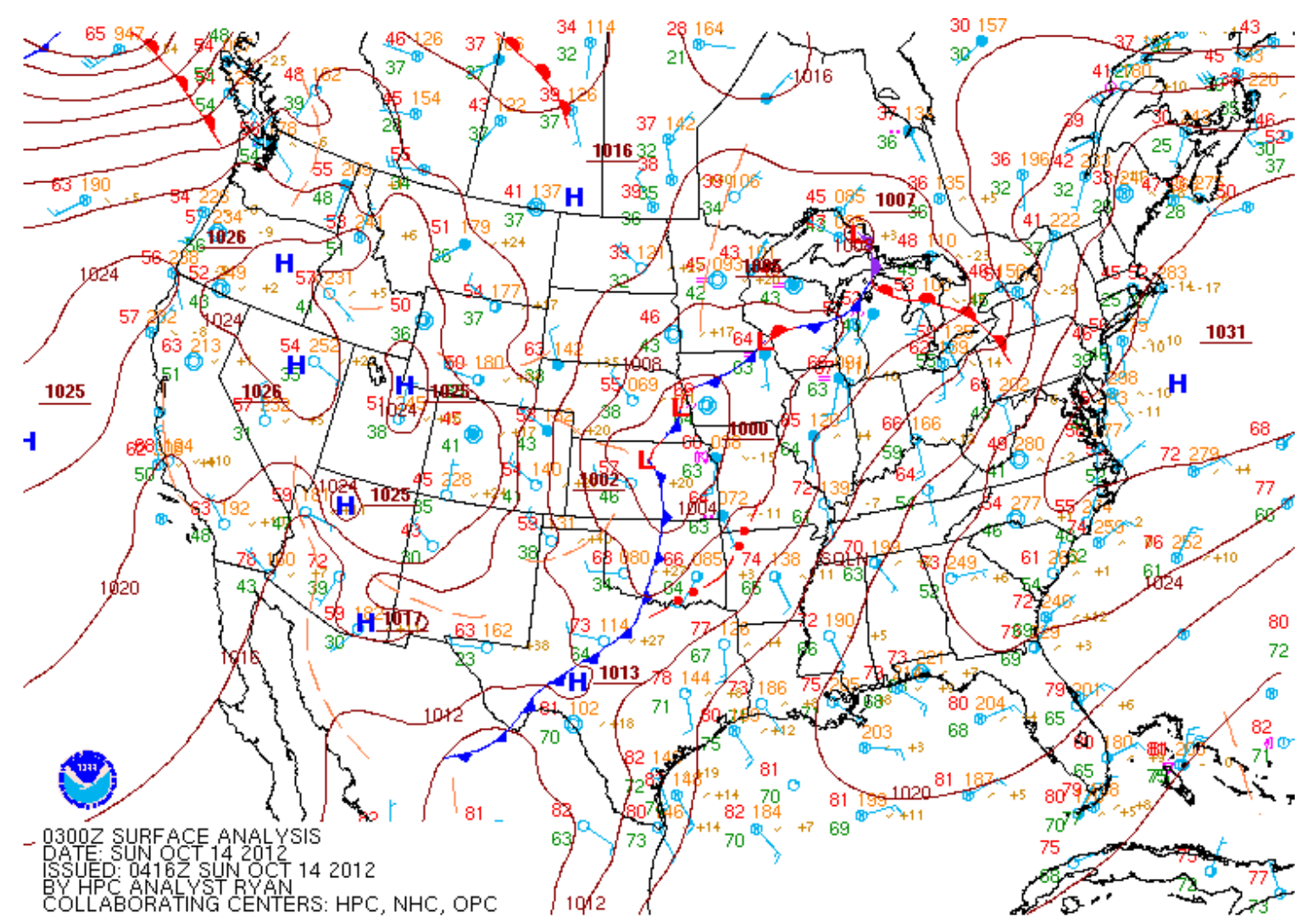

Figure 3.8.1. Surface Analysis 0300 UTC October 14, 2012.

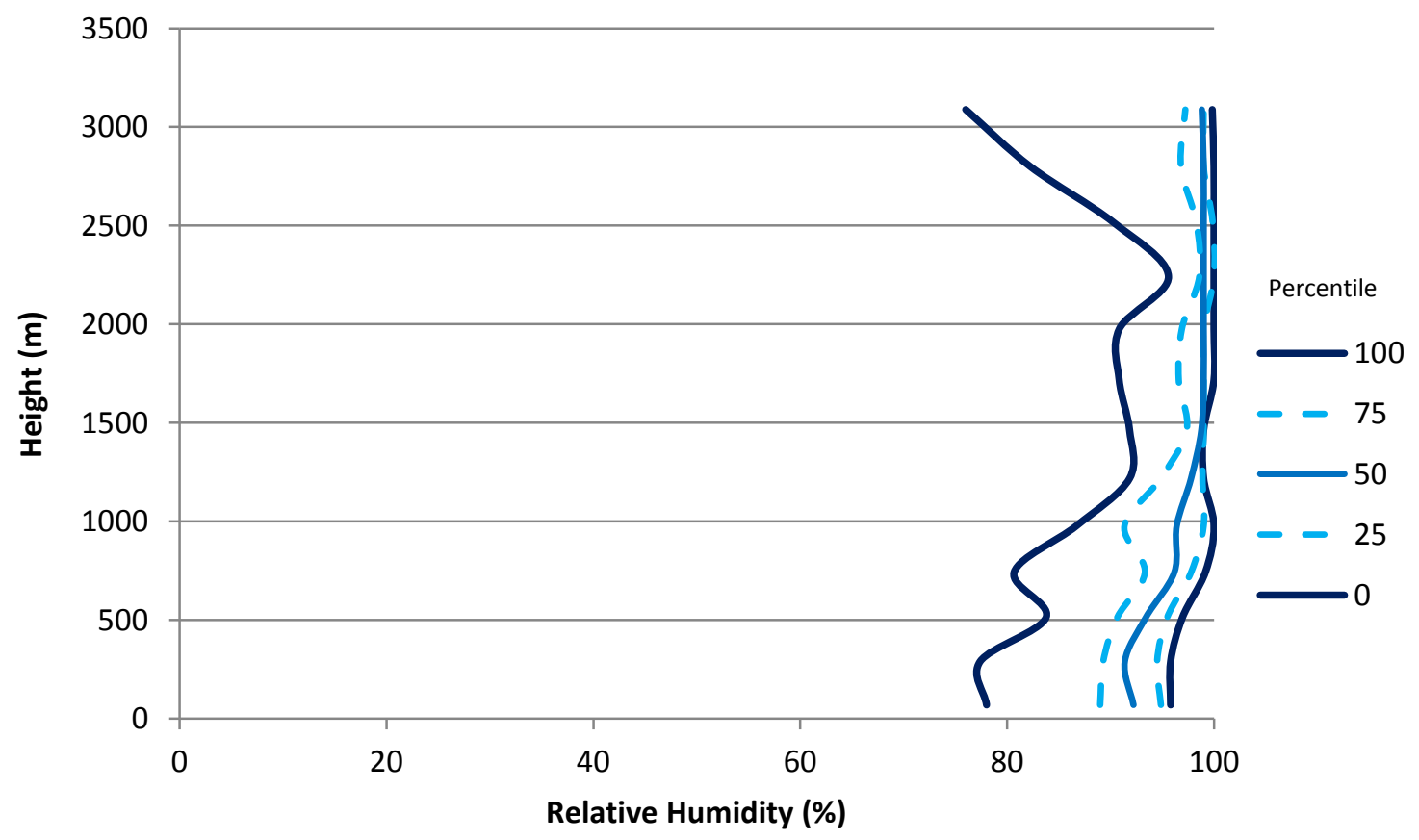

Figure 3.8.2. Relative Humidity Profile October 13, 2012. 


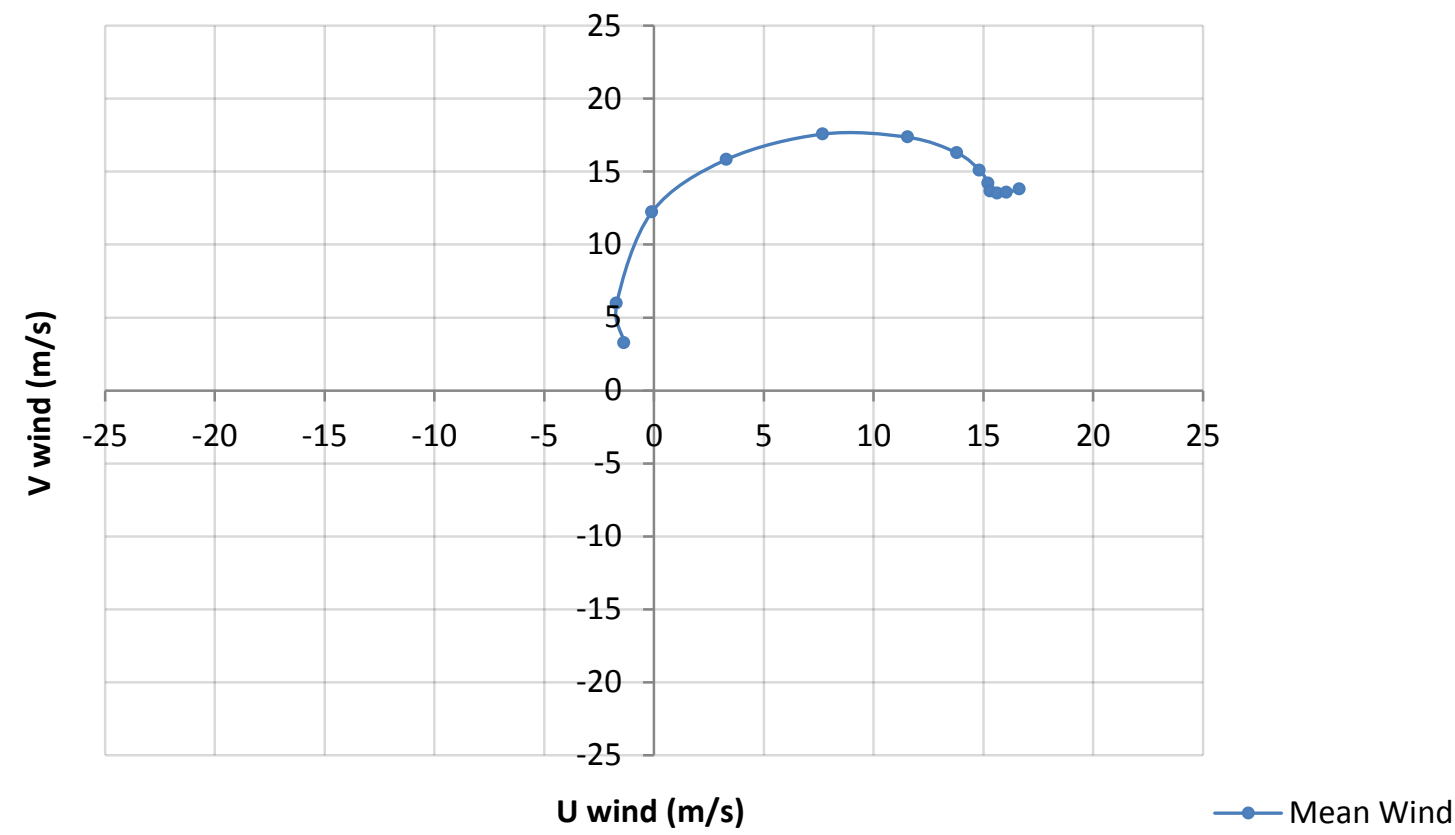

Figure 3.8.3. Hodograph October 13, 2012. 
Table 3.8.1 Conventional Techniques, October 13, 2012

\begin{tabular}{|c|c|c|c|c|c|}
\hline$Z=a R^{b}$ & \# & $\mathrm{FB}(\%)$ & $\mathrm{FAD}(\%)$ & FRMSE(\%) & FSD(\%) \\
\hline 1 & 72 & 14.67 & 42.26 & 60.87 & 59.08 \\
\hline 2 & 72 & 14.86 & 44.48 & 63.63 & 61.87 \\
\hline 3 & 72 & 91.72 & 93.74 & 141.29 & 107.47 \\
\hline 4 & 72 & 7.66 & 42.33 & 60.89 & 60.41 \\
\hline 5 & 72 & -24.11 & 45.74 & 67.66 & 63.22 \\
\hline \multicolumn{6}{|c|}{$R\left(K_{D P}\right)=a\left|K_{D P}\right|^{b} \operatorname{sign}\left(K_{D P}\right)$} \\
\hline 6 & 60 & 55.39 & 130.82 & 167.17 & 157.73 \\
\hline 7 & 60 & 74.51 & 144.89 & 185.89 & 170.31 \\
\hline 8 & 60 & 84.04 & 148.60 & 191.02 & 171.54 \\
\hline 9 & 60 & 38.99 & 117.41 & 148.64 & 143.43 \\
\hline 10 & 60 & 60.62 & 133.22 & 170.33 & 159.18 \\
\hline 11 & 60 & 54.50 & 127.78 & 162.40 & 152.99 \\
\hline \multicolumn{6}{|c|}{$R\left(Z, Z_{D R}\right)=a Z^{b} Z_{d r}^{c}$} \\
\hline 12 & 72 & 33.71 & 51.96 & 79.46 & 71.95 \\
\hline 13 & 72 & 38.88 & 56.61 & 92.41 & 83.83 \\
\hline 14 & 72 & 4.64 & 39.78 & 58.62 & 58.44 \\
\hline 15 & 72 & 1.41 & 39.34 & 58.12 & 58.10 \\
\hline 16 & 72 & 1.64 & 39.34 & 58.31 & 58.29 \\
\hline \multicolumn{6}{|c|}{$R\left(K_{D P}, Z_{D R}\right)=a\left|K_{D P}\right|^{b} Z_{d r}^{c} \operatorname{sign}\left(K_{D P}\right)$} \\
\hline 17 & 60 & 90.31 & 183.23 & 238.72 & 220.98 \\
\hline 18 & 62 & 121.69 & 238.29 & 319.96 & 295.92 \\
\hline 19 & 60 & 52.60 & 132.66 & 168.86 & 160.46 \\
\hline 20 & 60 & 77.35 & 155.93 & 200.16 & 184.61 \\
\hline \multicolumn{6}{|l|}{ Synthetic } \\
\hline 21 & 57 & 33.78 & 60.21 & 102.89 & 97.19 \\
\hline
\end{tabular}


Table 3.8.2 Trace Techniques, October 13, 2012

\begin{tabular}{|c|c|c|c|c|c|c|}
\hline & $Z_{D R}+$ & $\#$ & $\mathrm{FB}(\%)$ & FAD(\%) & FRMSE(\%) & FSD(\%) \\
\hline \multirow[t]{14}{*}{$\mathrm{T} 1$} & 0 & 72 & 187.44 & 187.46 & 247.60 & 161.79 \\
\hline & 0.2 & 72 & 151.81 & 151.87 & 203.73 & 135.86 \\
\hline & 0.3 & 72 & 134.66 & 134.75 & 182.82 & 123.65 \\
\hline & 0.4 & 72 & 118.40 & 118.52 & 163.24 & 112.37 \\
\hline & 0.5 & 72 & 103.44 & 103.57 & 145.70 & 102.61 \\
\hline & 0.6 & 72 & 89.77 & 89.96 & 129.94 & 93.95 \\
\hline & 0.7 & 72 & 77.37 & 77.81 & 116.16 & 86.64 \\
\hline & 0.8 & 72 & 66.11 & 67.00 & 104.05 & 80.35 \\
\hline & 0.9 & 72 & 55.78 & 58.14 & 93.29 & 74.78 \\
\hline & 1 & 72 & 46.47 & 51.52 & 83.97 & 69.94 \\
\hline & 1.1 & 72 & 37.90 & 46.74 & 75.60 & 65.42 \\
\hline & 1.5 & 72 & 10.36 & 37.30 & 54.86 & 53.87 \\
\hline & 2 & 72 & -14.82 & 35.77 & 51.65 & 49.48 \\
\hline & 2.6 & 72 & -35.65 & 44.97 & 63.30 & 52.31 \\
\hline \multirow[t]{12}{*}{$\mathrm{T} 2$} & 0.4 & 72 & 153.35 & 154.20 & 225.54 & 165.37 \\
\hline & 0.6 & 72 & 116.99 & 120.26 & 184.15 & 142.22 \\
\hline & 0.8 & 72 & 87.27 & 95.41 & 150.71 & 122.88 \\
\hline & 1 & 72 & 63.45 & 77.70 & 124.77 & 107.43 \\
\hline & 1.2 & 72 & 44.62 & 65.81 & 106.54 & 96.75 \\
\hline & 1.4 & 72 & 28.44 & 57.03 & 91.45 & 86.92 \\
\hline & 1.6 & 72 & 15.10 & 53.14 & 81.48 & 80.07 \\
\hline & 1.8 & 72 & 3.57 & 50.55 & 74.91 & 74.83 \\
\hline & 2 & 72 & -6.50 & 49.11 & 71.16 & 70.86 \\
\hline & 2.2 & 72 & -15.23 & 49.53 & 69.80 & 68.12 \\
\hline & 2.4 & 72 & -22.87 & 50.36 & 70.23 & 66.40 \\
\hline & 2.6 & 72 & -29.60 & 51.62 & 71.86 & 65.48 \\
\hline \multirow[t]{14}{*}{ T3 } & 0 & 72 & 184.40 & 184.40 & 246.65 & 163.80 \\
\hline & 0.2 & 72 & 147.79 & 147.87 & 201.19 & 136.51 \\
\hline & 0.3 & 72 & 130.46 & 130.63 & 179.96 & 123.96 \\
\hline & 0.4 & 72 & 114.44 & 114.70 & 160.90 & 113.11 \\
\hline & 0.5 & 72 & 99.91 & 100.49 & 144.19 & 103.96 \\
\hline & 0.6 & 72 & 86.45 & 87.38 & 129.02 & 95.77 \\
\hline & 0.7 & 72 & 74.24 & 76.10 & 115.65 & 88.68 \\
\hline & 0.8 & 72 & 62.97 & 66.62 & 103.60 & 82.26 \\
\hline & 0.9 & 72 & 52.78 & 59.18 & 93.06 & 76.64 \\
\hline & 1 & 72 & 43.71 & 54.02 & 84.30 & 72.09 \\
\hline & 1.1 & 72 & 35.56 & 49.85 & 77.14 & 68.45 \\
\hline & 1.5 & 72 & 8.72 & 40.75 & 58.96 & 58.31 \\
\hline & 2 & 72 & -15.38 & 40.91 & 56.67 & 54.55 \\
\hline & 2.6 & 72 & -35.71 & 47.92 & 66.67 & 56.31 \\
\hline
\end{tabular}


Table 3.8.2 Trace Techniques, October 13, 2012 (Continued)

\begin{tabular}{|c|c|c|c|c|c|c|}
\hline & $Z_{D R}+$ & $\#$ & $\mathrm{FB}(\%)$ & FAD(\%) & FRMSE(\%) & FSD(\%) \\
\hline \multirow[t]{12}{*}{ T4 } & 0.4 & 72 & 151.80 & 151.85 & 207.52 & 141.49 \\
\hline & 0.6 & 72 & 115.80 & 116.23 & 165.53 & 118.28 \\
\hline & 0.8 & 72 & 86.33 & 87.59 & 132.09 & 99.97 \\
\hline & 1 & 72 & 62.69 & 67.19 & 106.26 & 85.80 \\
\hline & 1.2 & 72 & 43.97 & 55.10 & 87.87 & 76.08 \\
\hline & 1.4 & 72 & 27.84 & 46.88 & 72.69 & 67.15 \\
\hline & 1.6 & 72 & 14.55 & 42.84 & 63.45 & 61.76 \\
\hline & 1.8 & 72 & 3.07 & 40.21 & 58.23 & 58.15 \\
\hline & 2 & 72 & -6.94 & 40.19 & 56.32 & 55.89 \\
\hline & 2.2 & 72 & -15.63 & 41.24 & 57.01 & 54.83 \\
\hline & 2.4 & 72 & -23.23 & 43.22 & 59.43 & 54.70 \\
\hline & 2.6 & 72 & -29.92 & 45.59 & 62.82 & 55.24 \\
\hline \multirow[t]{12}{*}{ T5 } & 0.4 & 72 & 157.73 & 157.81 & 212.56 & 142.49 \\
\hline & 0.6 & 72 & 120.42 & 120.53 & 167.96 & 117.08 \\
\hline & 0.8 & 72 & 90.42 & 90.87 & 133.53 & 98.26 \\
\hline & 1 & 72 & 66.10 & 67.69 & 106.67 & 83.72 \\
\hline & 1.2 & 72 & 46.33 & 52.87 & 85.54 & 71.90 \\
\hline & 1.4 & 72 & 30.14 & 45.07 & 70.22 & 63.42 \\
\hline & 1.6 & 72 & 16.20 & 40.22 & 59.36 & 57.11 \\
\hline & 1.8 & 72 & 4.20 & 37.96 & 53.04 & 52.87 \\
\hline & 2 & 72 & -6.10 & 36.74 & 50.85 & 50.48 \\
\hline & 2.2 & 72 & -15.01 & 36.13 & 51.82 & 49.59 \\
\hline & 2.4 & 72 & -22.80 & 37.91 & 54.75 & 49.78 \\
\hline & 2.6 & 72 & -29.67 & 40.97 & 58.74 & 50.70 \\
\hline
\end{tabular}




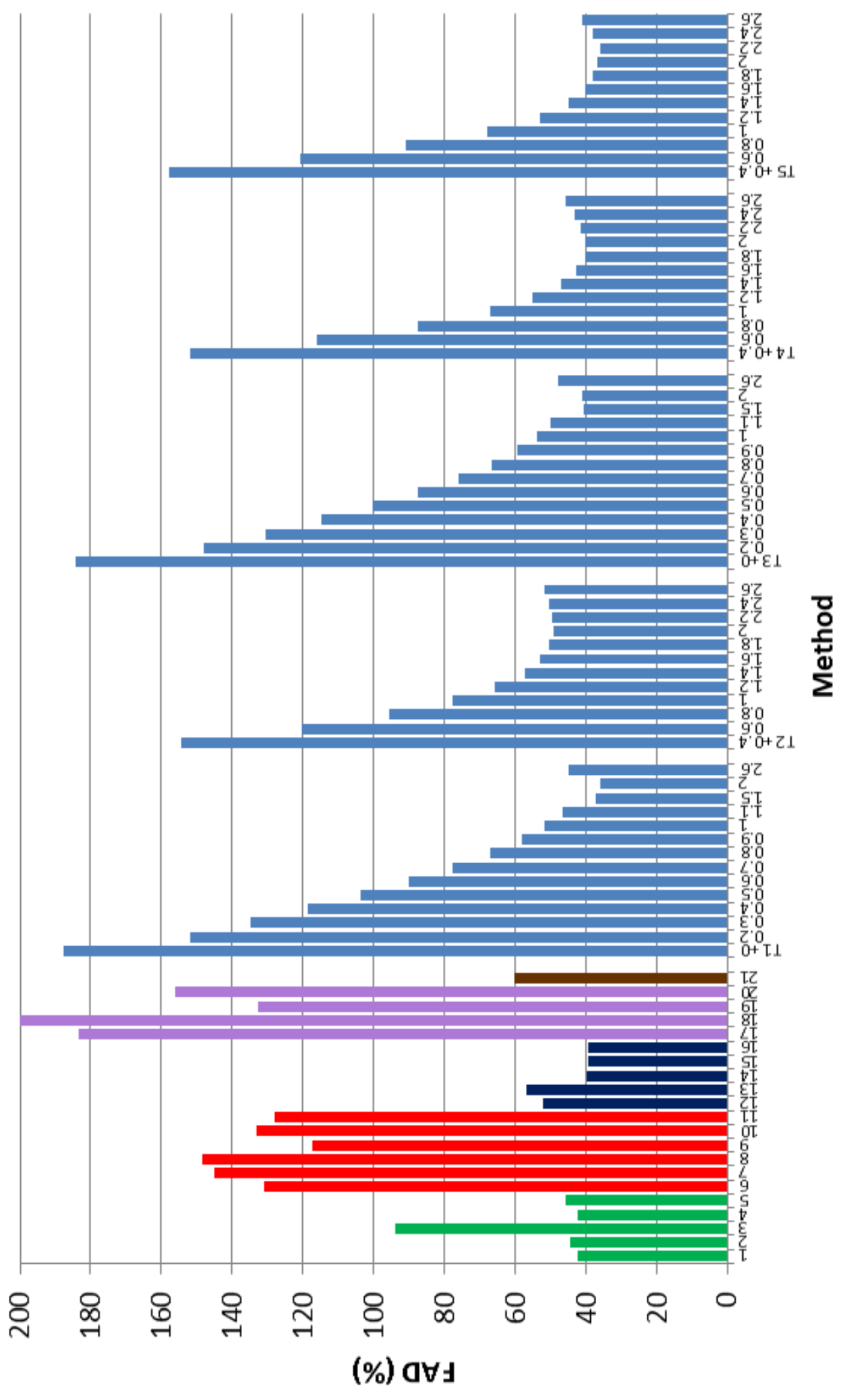

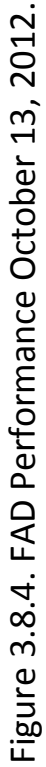




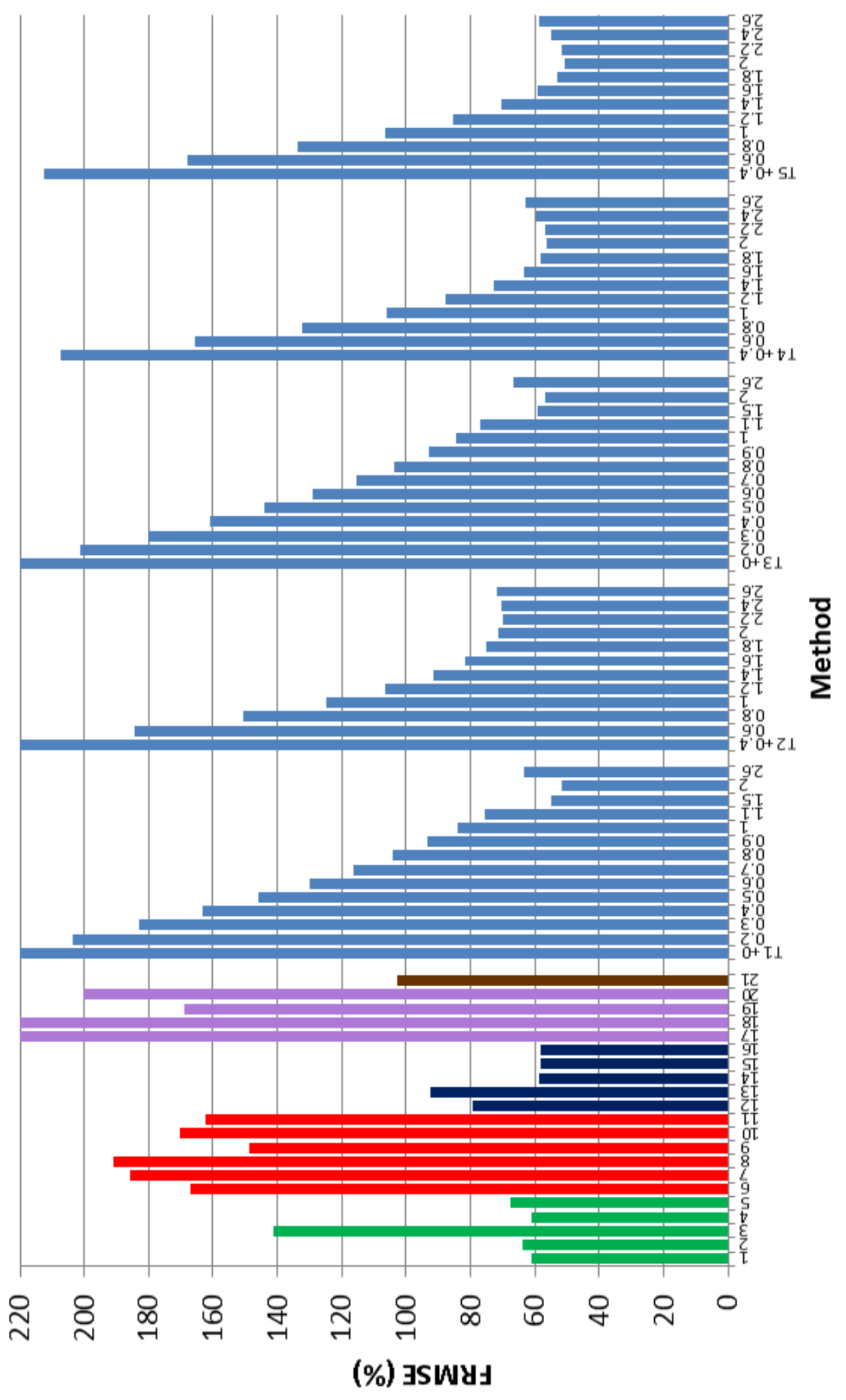

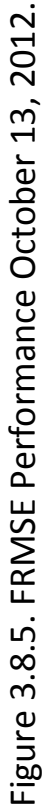




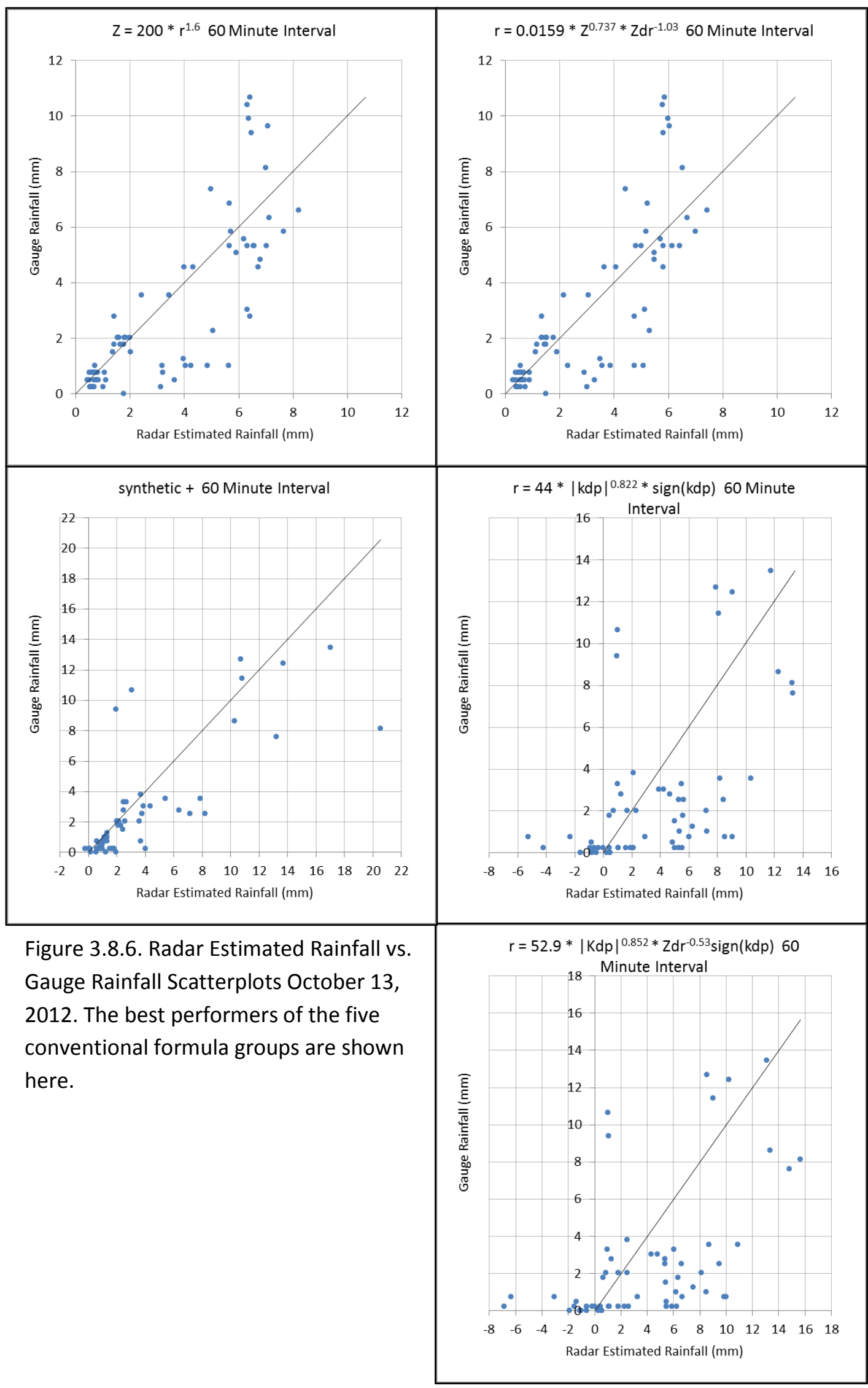




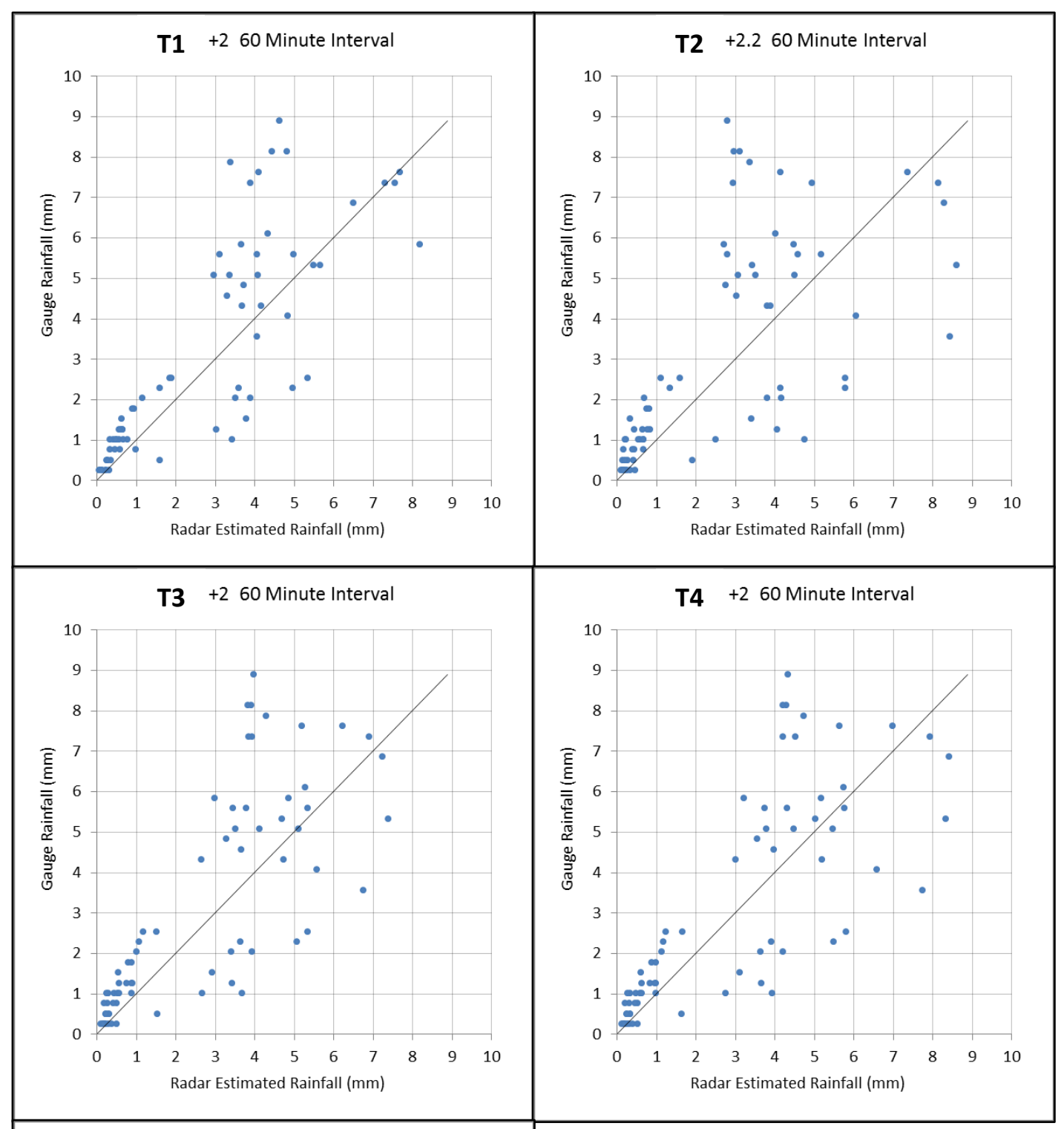

T5 +2 60 Minute Interval

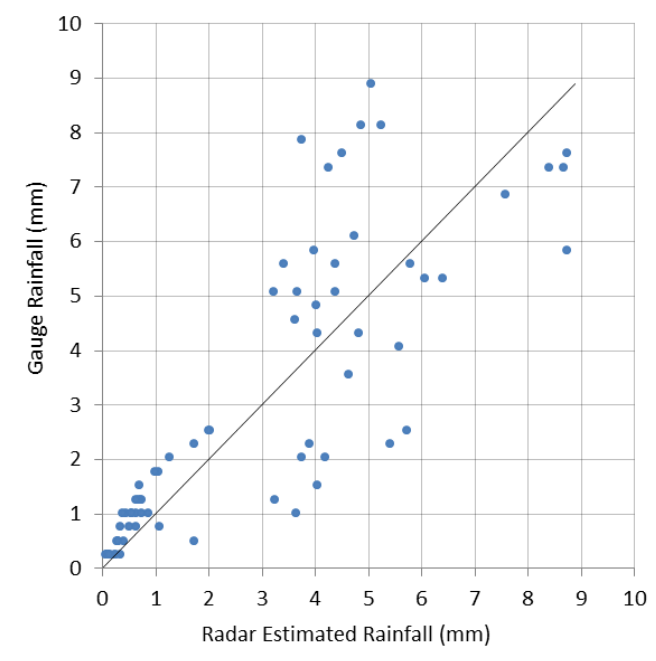

Figure 3.8.7. Radar Estimated Rainfall vs. Gauge Rainfall Scatterplots October 13, 2012. The best performers of the five trace techniques are shown here. 


\subsection{March 10, 2013}

The rainfall event on March 10 took place to the east of an occluded low pressure center and several associated fronts. Figure 3.9.1 is an HPC surface analysis for 06 UTC of March, 2012. Figure 3.9.2 shows humidity versus height in percentile format for 01 UTC through 11 UTC of March 10. The atmosphere was very dry near the surface initially, but moistened as the event progressed. Figure 3.9 .3 is a hodograph for the $13^{\text {th }}$ of October. The hodograph shows a southerly wind at the surface turning southwesterly higher up. Figure 3.9.4 is a four panel radar image from KLSX for 0631 UTC of March 10.

\subsubsection{Conventional Formulas}

Table 3.9.1 shows the performance of the conventional formulas for this event. (1), (2), and (4) performed the best out of the conventional formulas, and as a whole the Z-R formulas performed better than the $\mathrm{Z}, \mathrm{Z}_{\mathrm{DR}}-\mathrm{R}$ formulas. All of the $\mathrm{K}_{\mathrm{DP}}$-reliant formulas performed very poorly for this date. The $\mathrm{K}_{\mathrm{DP}}$-reliant formulas exhibited very positive biases, while the other conventional formulas tended to have negative biases.

\subsubsection{Tracing Techniques}

Table 3.9.2 shows the performance of the various trace techniques and their permutations for this event. Techniques $T 2, T 4$, and $T 5$ were most effective at the $+Z_{D R}$ values of around 1.4, while $T 1$ and $T 3$ were most effective at $+Z_{D R}$ values near 1 . The optimal tracing techniques performed similarly to the non- $K_{D P}$ reliant conventional formulas for this event. 


\subsubsection{Graphical Evaluation}

Figure 3.9.5 is a histogram showing the FAD performance of each evaluated technique for this event. Figure 3.9.6 is the equivalent histogram showing FRMSE performance.

Figure 3.9.7 shows a combination of scatterplots of radar-derived rainfall versus rain gauge rainfall for the conventional formulas. The techniques illustrated were the top performers in each category. Figure 3.9.8 is identical to 3.9.7, except it depicts the best performers of the trace techniques. 


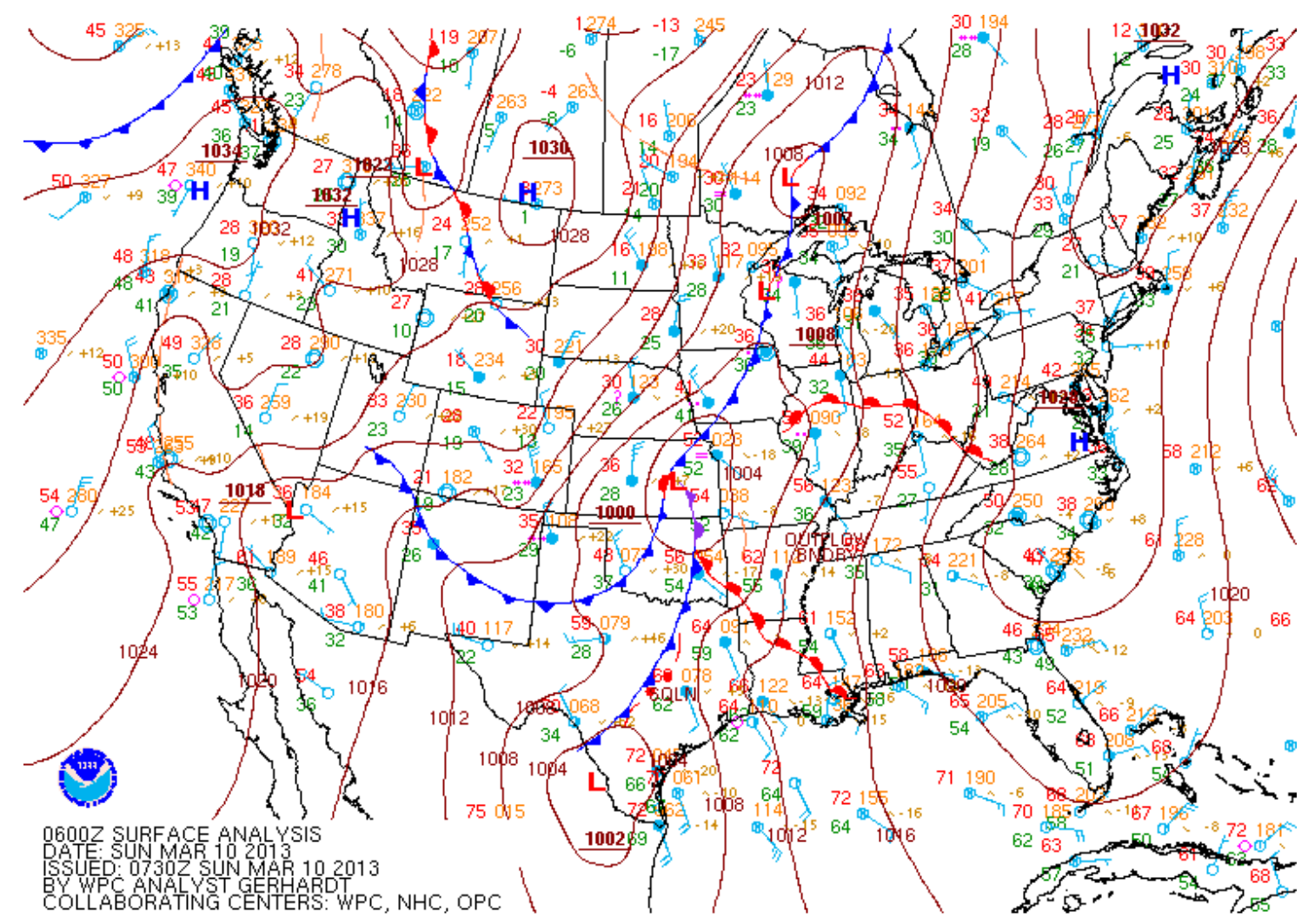

Figure 3.9.1. Surface Analysis 0600 UTC March 10, 2013.

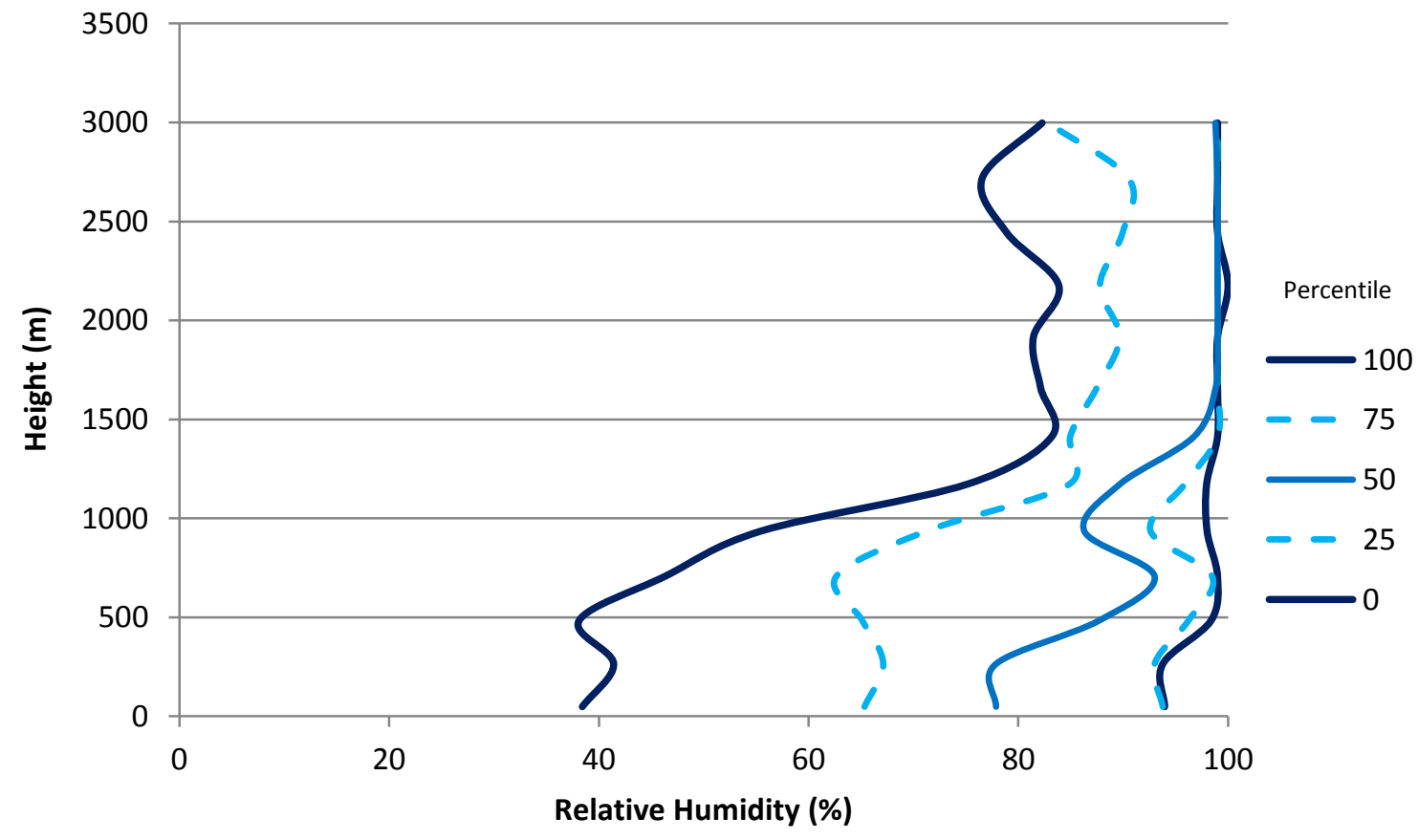

Figure 3.9.2. Relative Humidity Profile March 10, 2013. 


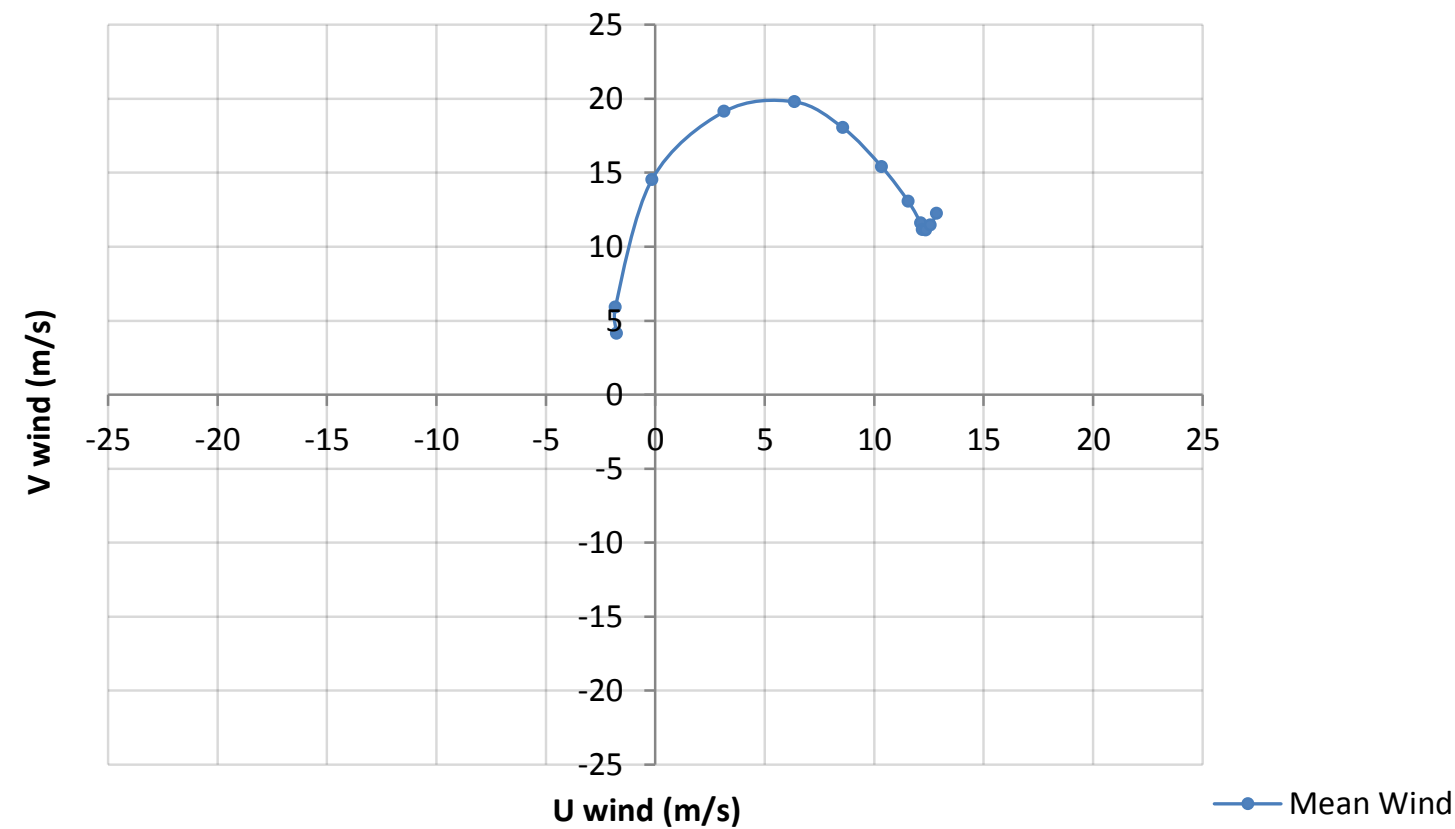

Figure 3.9.3. Hodograph March 10, 2013. 


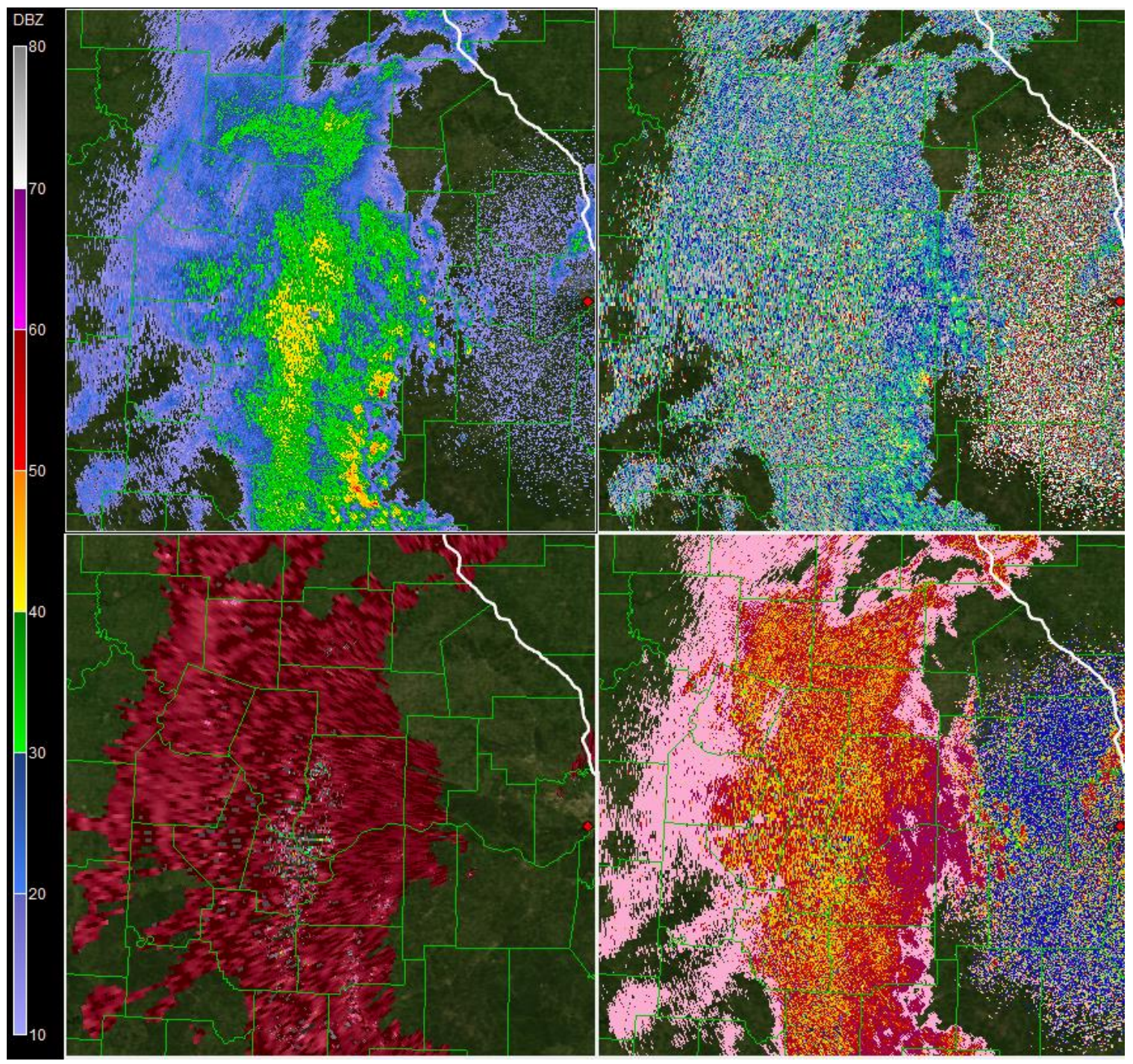

Figure 3.9.4. Four Panel Radar Image for 0631 UTC March 10, 2013. The top left is reflectivity $(Z)$, top right is differential reflectivity $\left(Z_{D R}\right)$, bottom left is specific phase differential $\left(K_{D P}\right)$, and bottom right is correlation coefficient. The scale on the left corresponds to the top left image ( $\mathrm{Z})$. The elevation angle used here was $0.5^{\circ}$. 
Table 3.9.1 Conventional Techniques, March 10, 2013

\begin{tabular}{|c|c|c|c|c|c|}
\hline$Z=a R^{b}$ & \# & $\mathrm{FB}(\%)$ & $\mathrm{FAD}(\%)$ & FRMSE(\%) & FSD(\%) \\
\hline 1 & 45 & -2.68 & 47.73 & 55.54 & 55.48 \\
\hline 2 & 45 & -13.28 & 47.45 & 57.40 & 55.84 \\
\hline 3 & 45 & 22.93 & 57.41 & 69.13 & 65.21 \\
\hline 4 & 45 & -16.54 & 47.20 & 57.62 & 55.19 \\
\hline 5 & 45 & -42.32 & 51.10 & 68.21 & 53.49 \\
\hline \multicolumn{6}{|c|}{$R\left(K_{D P}\right)=a\left|K_{D P}\right|^{b} \operatorname{sign}\left(K_{D P}\right)$} \\
\hline 6 & 52 & 63.19 & 212.88 & 278.71 & 271.45 \\
\hline 7 & 52 & 78.86 & 235.74 & 308.69 & 298.44 \\
\hline 8 & 52 & 78.98 & 240.24 & 311.52 & 301.35 \\
\hline 9 & 51 & 43.59 & 188.04 & 241.39 & 237.43 \\
\hline 10 & 52 & 65.16 & 216.95 & 283.07 & 275.47 \\
\hline 11 & 52 & 57.05 & 206.75 & 268.57 & 262.45 \\
\hline \multicolumn{6}{|c|}{$R\left(Z, Z_{D R}\right)=a Z^{b} Z_{d r}^{c}$} \\
\hline 12 & 45 & 6.41 & 59.47 & 70.50 & 70.20 \\
\hline 13 & 45 & 24.86 & 69.76 & 86.29 & 82.63 \\
\hline 14 & 45 & -14.40 & 49.15 & 60.23 & 58.49 \\
\hline 15 & 45 & -18.04 & 48.18 & 59.49 & 56.69 \\
\hline 16 & 45 & -17.06 & 48.74 & 60.21 & 57.75 \\
\hline \multicolumn{6}{|c|}{$R\left(K_{D P}, Z_{D R}\right)=a\left|K_{D P}\right|^{b} Z_{d r}^{c} \operatorname{sign}\left(K_{D P}\right)$} \\
\hline 17 & 53 & 142.18 & 326.26 & 424.72 & 400.22 \\
\hline 18 & 53 & 224.51 & 452.05 & 579.75 & 534.51 \\
\hline 19 & 53 & 65.77 & 217.09 & 284.59 & 276.88 \\
\hline 20 & 53 & 95.78 & 258.59 & 340.27 & 326.51 \\
\hline \multicolumn{6}{|l|}{ Synthetic } \\
\hline 21 & 45 & 5.25 & 59.62 & 87.58 & 87.42 \\
\hline
\end{tabular}


Table 3.9.2 Trace Techniques, March 10, 2013

\begin{tabular}{|c|c|c|c|c|c|c|}
\hline & $Z_{D R}+$ & $\#$ & $\mathrm{FB}(\%)$ & FAD(\%) & FRMSE(\%) & $\mathrm{FSD}(\%)$ \\
\hline \multirow[t]{14}{*}{ T1 } & 0 & 45 & 53.66 & 71.04 & 87.90 & 69.62 \\
\hline & 0.2 & 45 & 40.63 & 64.79 & 76.94 & 65.34 \\
\hline & 0.3 & 45 & 33.73 & 61.60 & 71.72 & 63.30 \\
\hline & 0.4 & 45 & 26.81 & 58.33 & 66.89 & 61.28 \\
\hline & 0.5 & 45 & 19.90 & 55.36 & 62.90 & 59.67 \\
\hline & 0.6 & 45 & 13.17 & 52.97 & 60.20 & 58.74 \\
\hline & 0.7 & 45 & 6.65 & 50.61 & 58.26 & 57.88 \\
\hline & 0.8 & 45 & 0.26 & 48.35 & 56.98 & 56.98 \\
\hline & 0.9 & 45 & -5.85 & 46.58 & 56.49 & 56.19 \\
\hline & 1 & 45 & -11.42 & 44.99 & 56.72 & 55.55 \\
\hline & 1.1 & 45 & -16.72 & 43.94 & 57.65 & 55.17 \\
\hline & 1.5 & 45 & -33.70 & 44.77 & 63.89 & 54.29 \\
\hline & 2 & 45 & -49.58 & 51.84 & 72.24 & 52.54 \\
\hline & 2.6 & 45 & -62.32 & 62.51 & 80.40 & 50.80 \\
\hline \multirow[t]{12}{*}{$\mathrm{T} 2$} & 0.4 & 46 & 82.40 & 100.27 & 137.62 & 110.22 \\
\hline & 0.6 & 45 & 56.46 & 85.20 & 109.97 & 94.37 \\
\hline & 0.8 & 45 & 32.20 & 71.36 & 88.90 & 82.87 \\
\hline & 1 & 45 & 12.34 & 61.83 & 75.34 & 74.32 \\
\hline & 1.2 & 45 & -3.74 & 54.51 & 67.91 & 67.81 \\
\hline & 1.4 & 45 & -16.28 & 50.56 & 65.40 & 63.34 \\
\hline & 1.6 & 45 & -26.42 & 49.21 & 65.62 & 60.07 \\
\hline & 1.8 & 45 & -34.68 & 49.27 & 67.29 & 57.66 \\
\hline & 2 & 45 & -41.64 & 50.97 & 69.54 & 55.69 \\
\hline & 2.2 & 45 & -47.60 & 54.01 & 72.32 & 54.45 \\
\hline & 2.4 & 45 & -52.84 & 57.29 & 75.11 & 53.38 \\
\hline & 2.6 & 45 & -57.32 & 60.48 & 77.79 & 52.59 \\
\hline \multirow[t]{14}{*}{ T3 } & 0 & 45 & 54.23 & 67.86 & 93.93 & 76.70 \\
\hline & 0.2 & 45 & 39.23 & 60.77 & 82.41 & 72.47 \\
\hline & 0.3 & 45 & 32.00 & 58.43 & 77.52 & 70.60 \\
\hline & 0.4 & 45 & 24.81 & 56.18 & 72.87 & 68.52 \\
\hline & 0.5 & 45 & 17.68 & 54.10 & 68.68 & 66.37 \\
\hline & 0.6 & 45 & 10.76 & 52.00 & 65.28 & 64.39 \\
\hline & 0.7 & 45 & 3.74 & 49.55 & 62.35 & 62.24 \\
\hline & 0.8 & 45 & -2.93 & 47.65 & 60.31 & 60.24 \\
\hline & 0.9 & 45 & -9.15 & 46.16 & 59.25 & 58.54 \\
\hline & 1 & 45 & -14.87 & 45.41 & 59.05 & 57.15 \\
\hline & 1.1 & 45 & -20.19 & 45.23 & 59.47 & 55.94 \\
\hline & 1.5 & 45 & -37.15 & 48.19 & 64.54 & 52.78 \\
\hline & 2 & 45 & -51.71 & 54.90 & 72.46 & 50.75 \\
\hline & 2.6 & 45 & -63.78 & 64.89 & 80.79 & 49.58 \\
\hline
\end{tabular}


Table 3.9.2 Trace Techniques, March 10, 2013 (Continued)

\begin{tabular}{|c|c|c|c|c|c|c|}
\hline & $Z_{D R}+$ & $\#$ & $\mathrm{FB}(\%)$ & FAD(\%) & FRMSE(\%) & $\mathrm{FSD}(\%)$ \\
\hline \multirow[t]{12}{*}{ T4 } & 0.4 & 45 & 82.14 & 94.69 & 133.86 & 105.69 \\
\hline & 0.6 & 45 & 56.34 & 80.70 & 107.58 & 91.64 \\
\hline & 0.8 & 45 & 32.06 & 67.37 & 86.13 & 79.94 \\
\hline & 1 & 45 & 12.21 & 58.27 & 72.62 & 71.58 \\
\hline & 1.2 & 45 & -3.87 & 51.47 & 65.44 & 65.33 \\
\hline & 1.4 & 45 & -16.40 & 46.84 & 63.34 & 61.18 \\
\hline & 1.6 & 45 & -26.53 & 46.13 & 64.02 & 58.27 \\
\hline & 1.8 & 45 & -34.78 & 47.60 & 66.06 & 56.16 \\
\hline & 2 & 45 & -41.73 & 50.43 & 68.60 & 54.45 \\
\hline & 2.2 & 45 & -47.67 & 53.61 & 71.57 & 53.38 \\
\hline & 2.4 & 45 & -52.91 & 56.55 & 74.49 & 52.43 \\
\hline & 2.6 & 45 & -57.39 & 59.83 & 77.27 & 51.74 \\
\hline \multirow[t]{12}{*}{ T5 } & 0.4 & 45 & 88.12 & 101.79 & 129.18 & 94.46 \\
\hline & 0.6 & 45 & 62.53 & 86.22 & 105.64 & 85.15 \\
\hline & 0.8 & 45 & 39.28 & 73.94 & 87.07 & 77.70 \\
\hline & 1 & 45 & 18.86 & 63.06 & 73.26 & 70.79 \\
\hline & 1.2 & 45 & 2.08 & 55.45 & 66.62 & 66.58 \\
\hline & 1.4 & 45 & -10.89 & 50.75 & 65.00 & 64.08 \\
\hline & 1.6 & 45 & -21.89 & 47.99 & 65.32 & 61.54 \\
\hline & 1.8 & 45 & -31.19 & 47.05 & 67.11 & 59.42 \\
\hline & 2 & 45 & -38.94 & 47.70 & 69.43 & 57.49 \\
\hline & 2.2 & 45 & -45.38 & 50.18 & 71.98 & 55.88 \\
\hline & 2.4 & 45 & -50.83 & 53.20 & 74.57 & 54.57 \\
\hline & 2.6 & 45 & -55.48 & 57.10 & 77.11 & 53.56 \\
\hline
\end{tabular}




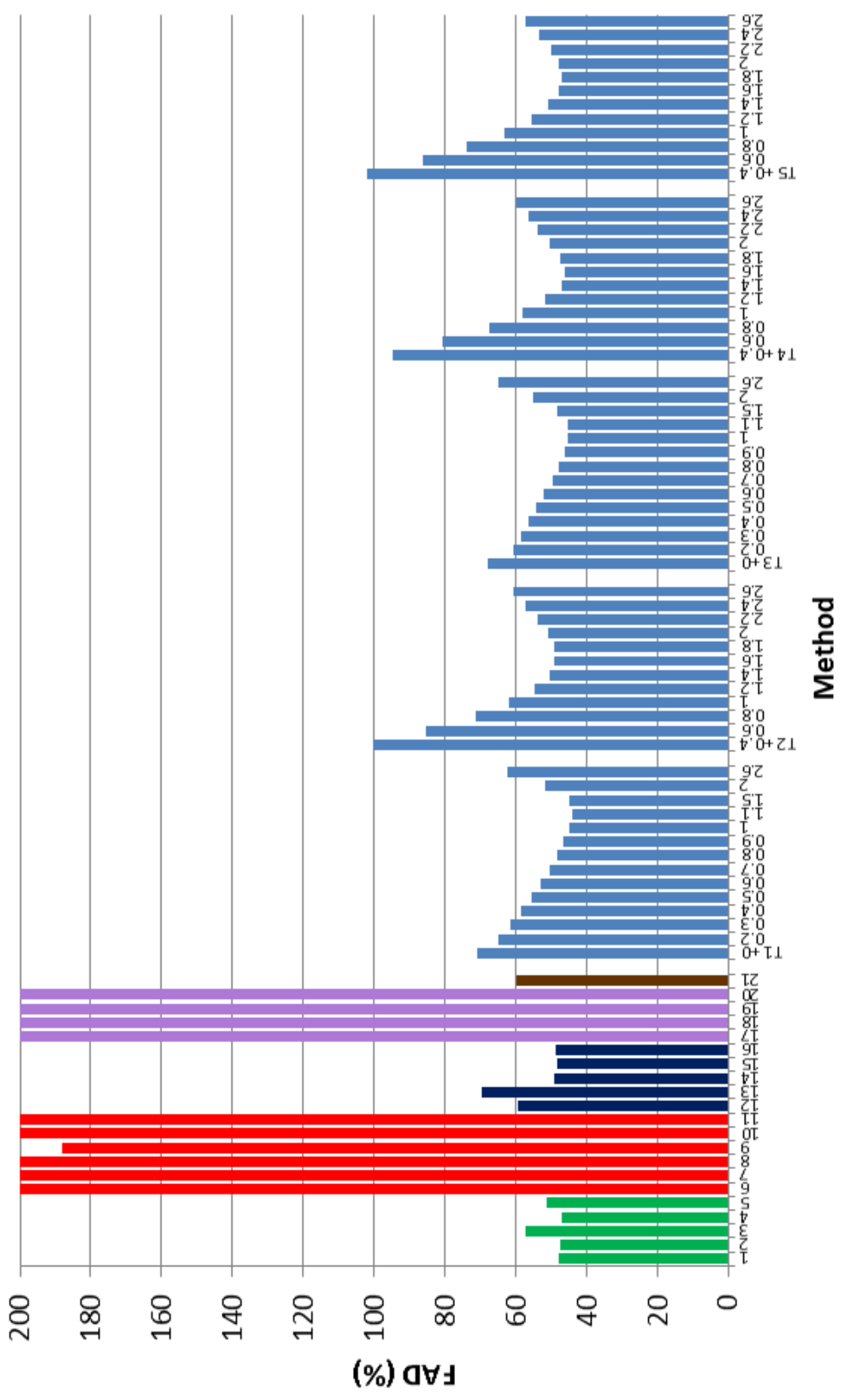

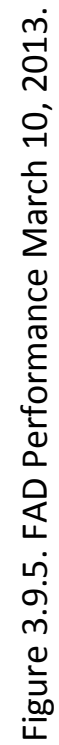




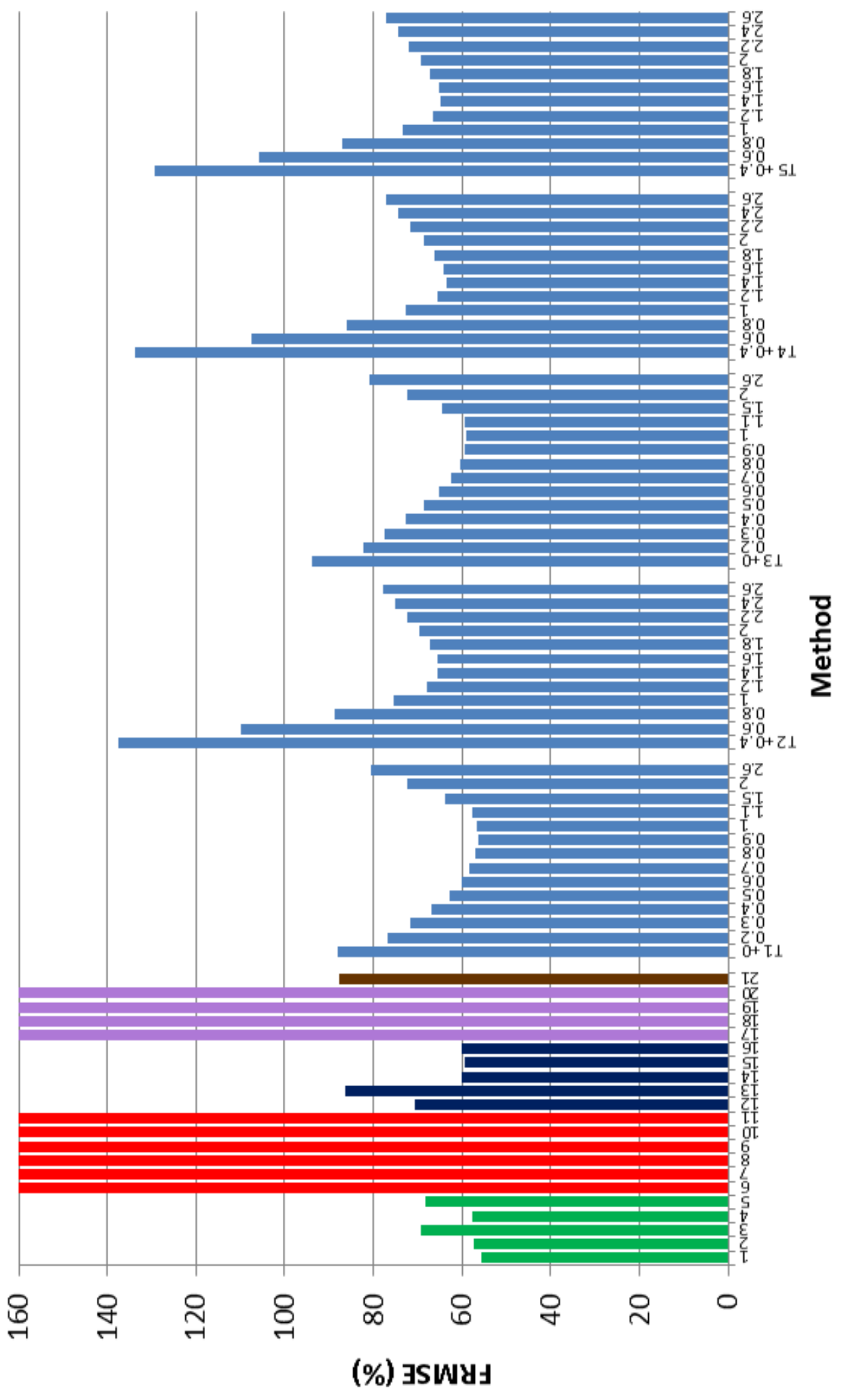

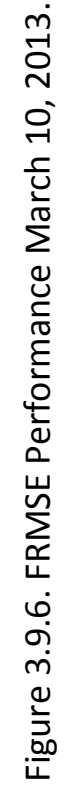




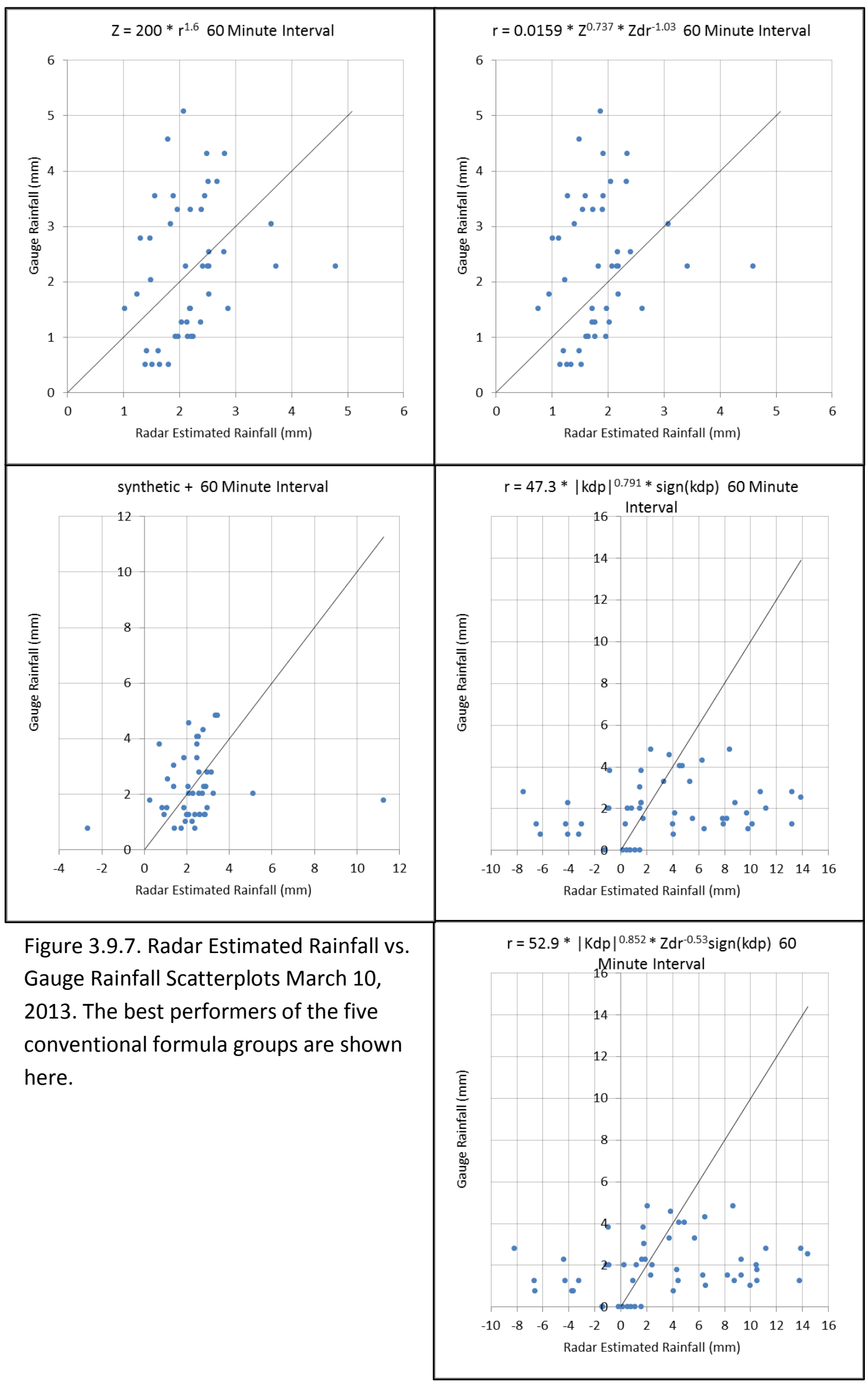




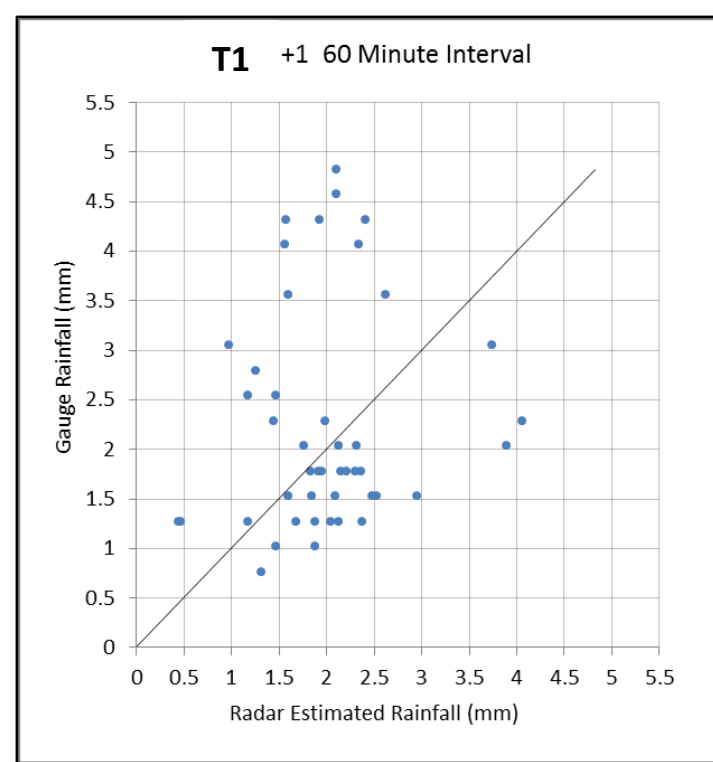

T3 +1 60 Minute Interval

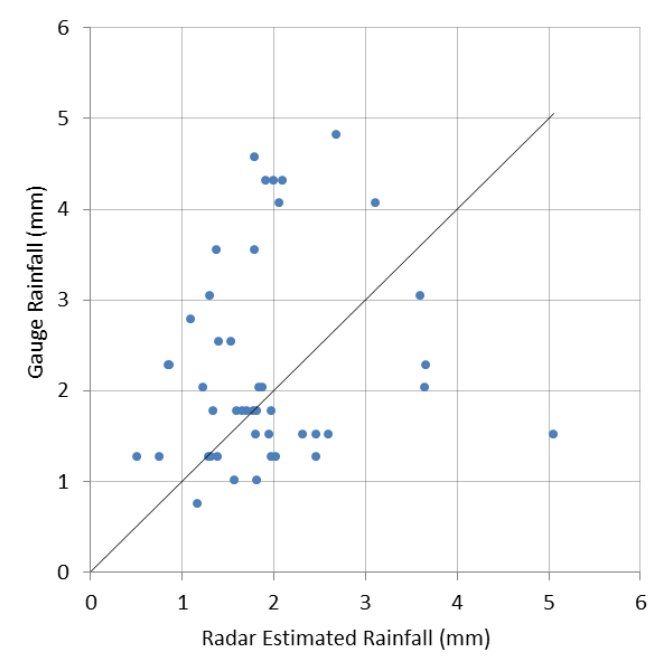

T5 +1.6 60 Minute Interval

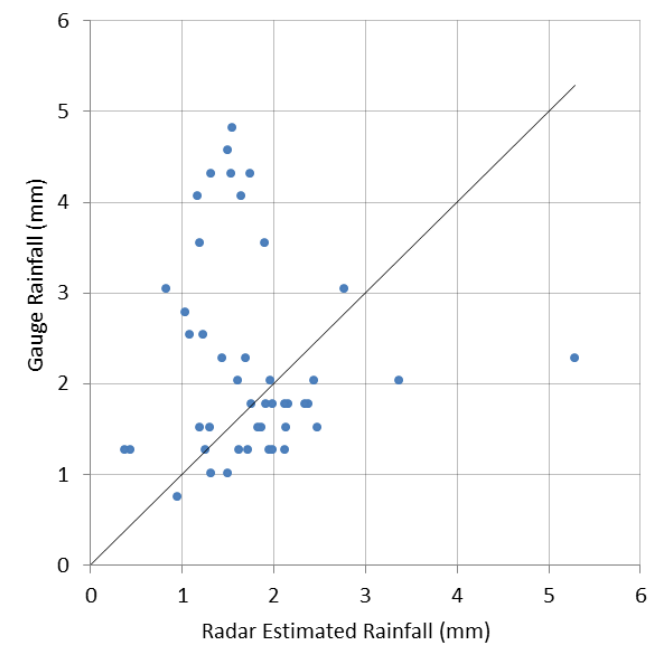

T2 +1.6 60 Minute Interval

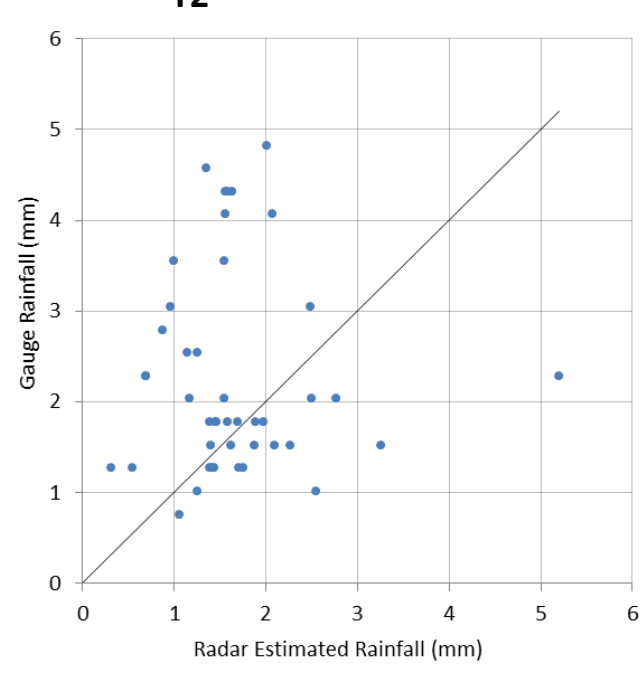

T4 +1.4 60 Minute Interval

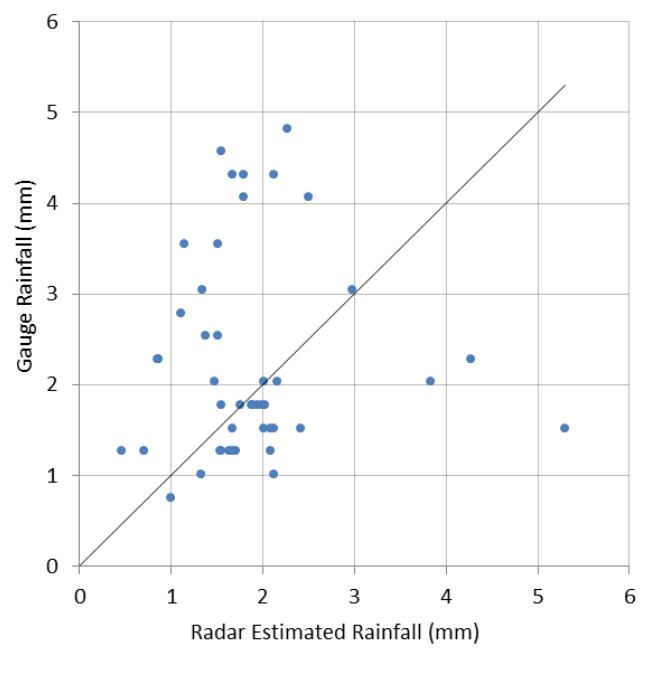

Figure 3.9.8. Radar Estimated Rainfall vs. Gauge Rainfall Scatterplots March 10, 2013. The best performers of the five trace techniques are shown here. 


\subsection{April 10, 2013}

The event on April 10 was associated with two low pressure centers almost directly on top of Columbia. Figure 3.10.1 is an HPC surface analysis for 21 UTC of April 10. Figure 3.10.2 shows humidity versus height in percentile format for 19 UTC of April 10 through 05 UTC of April 11. The atmosphere was moist at the lowest layers throughout the event. Initially a dry layer was present above 750 meters, but it quickly reached saturation as the event progressed. Figure 3.10.3 is a hodograph for the $10^{\text {th }}$ of April. The hodograph shows the wind direction turning clockwise with height, changing from northwesterly at the surface to southwesterly at 700 millibars. Figure 3.10 .4 is a four panel radar image from KLSX for 2154 UTC of April 10.

\subsubsection{Conventional Formulas}

Table 3.10.1 shows the performance of the conventional formulas for this event. The most basic Z-R relationship, (1), was the top performer of the conventional formulas. Of the non- $K_{D P}$ reliant formulas, three outliers were present, $\left(3,12\right.$, and 13 again). The $K_{D^{-}}$ reliant formulas again performed poorly.

\subsubsection{Tracing Techniques}

Table 3.10.2 shows the performance of the various trace techniques and their permutations for this event. Techniques $T 1$ and $T 5$ were most effective at $+Z_{D R}$ values of 2.6, while the other tracing techniques were most effective at $+Z_{D R}$ values of 2 . The first two in this case accounted for drift while the latter three did not. As a whole, the optimal tracing techniques performed slightly worse than the (non- $K_{D P}$ reliant) 
conventional formulas on this date. The April 10 event may have been another case where the modeled humidity was inaccurate (too high), due to the large optimal $+Z_{D R}$ values for T1 and T3.

\subsubsection{Graphical Evaluation}

Figure 3.10.5 is a histogram showing the FAD performance of each evaluated technique for this event. Figure 3.10.6 is the equivalent histogram showing FRMSE performance.

Figure 3.10.7 shows a combination of scatterplots of radar-derived rainfall versus rain gauge rainfall for the conventional formulas. The techniques illustrated were the top performers in each category. Figure 3.10.8 is identical to 3.10.7, except it depicts the best performers of the trace techniques. 


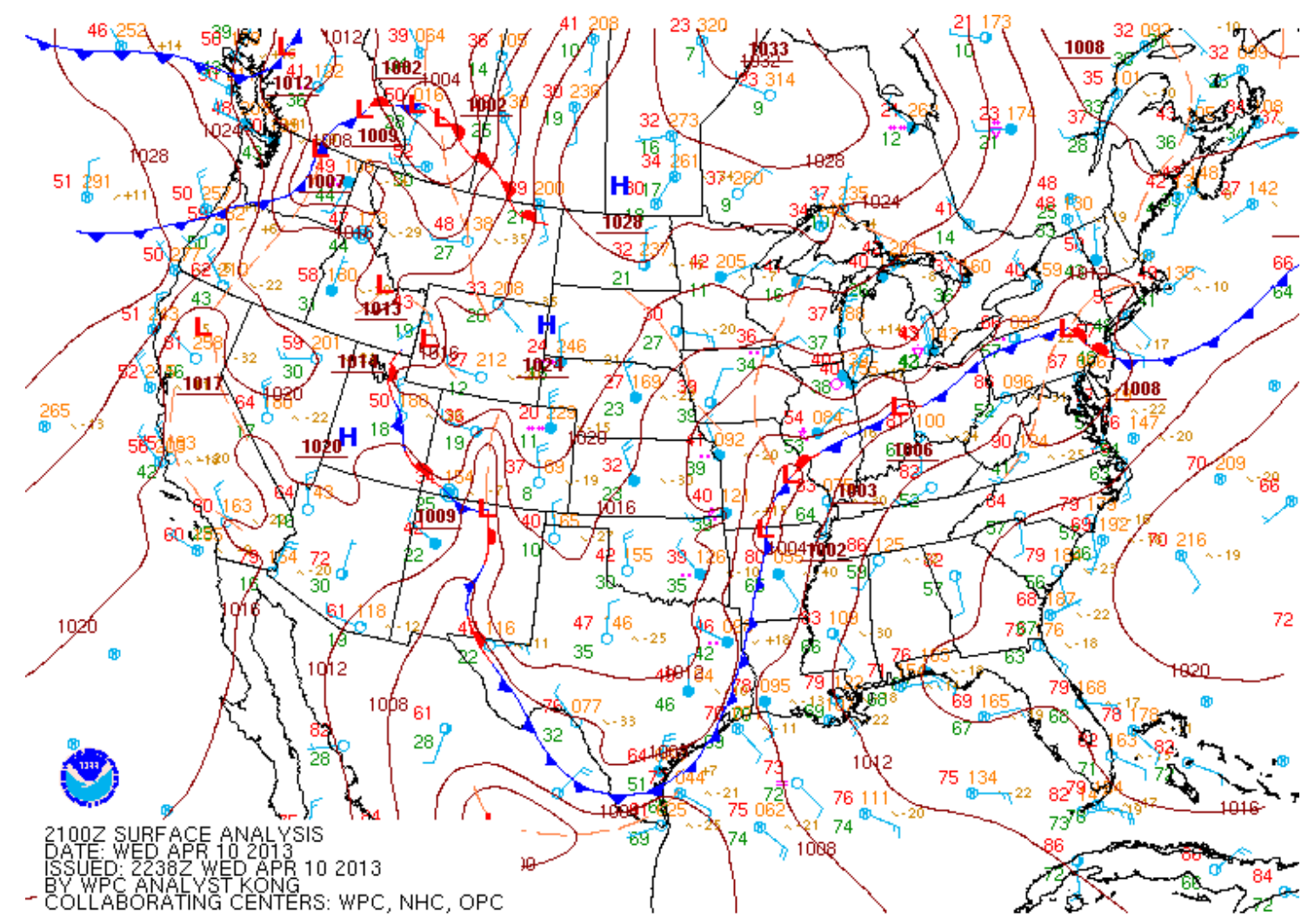

Figure 3.10.1. Surface Analysis 2100 UTC April 10, 2013.

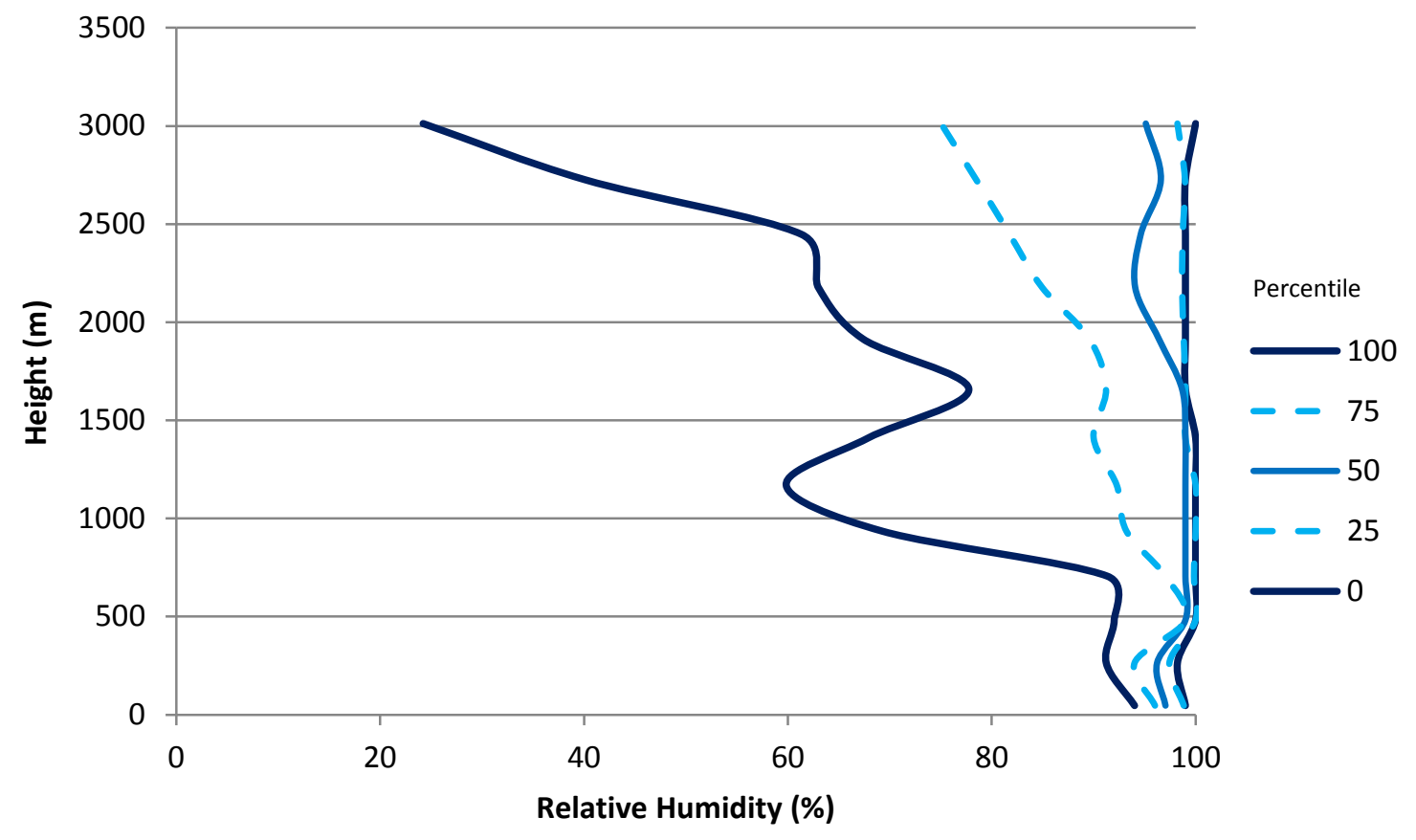

Figure 3.10.2. Relative Humidity Profile April 10, 2013. 


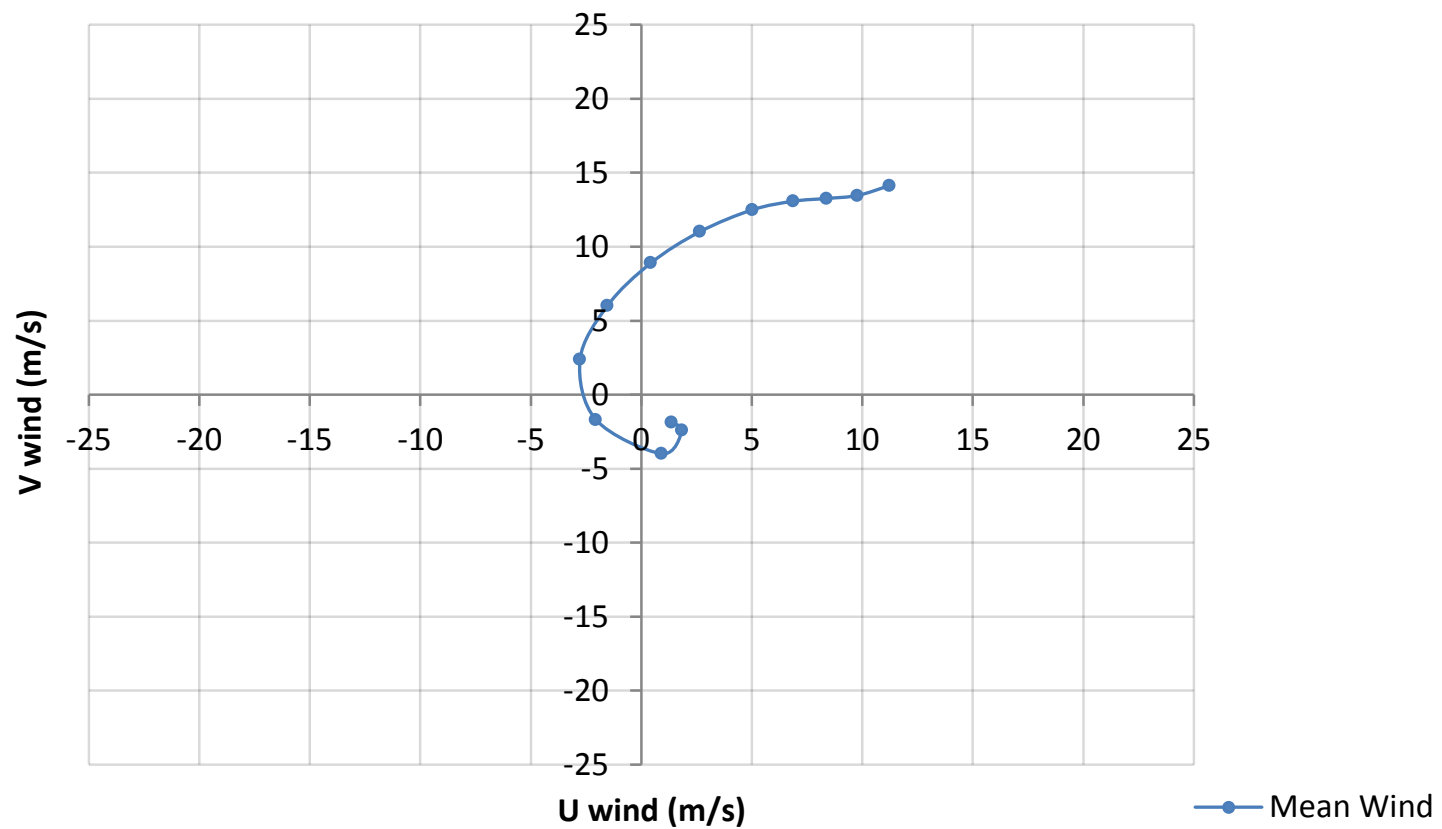

Figure 3.10.3. Hodograph April 10, 2013. 


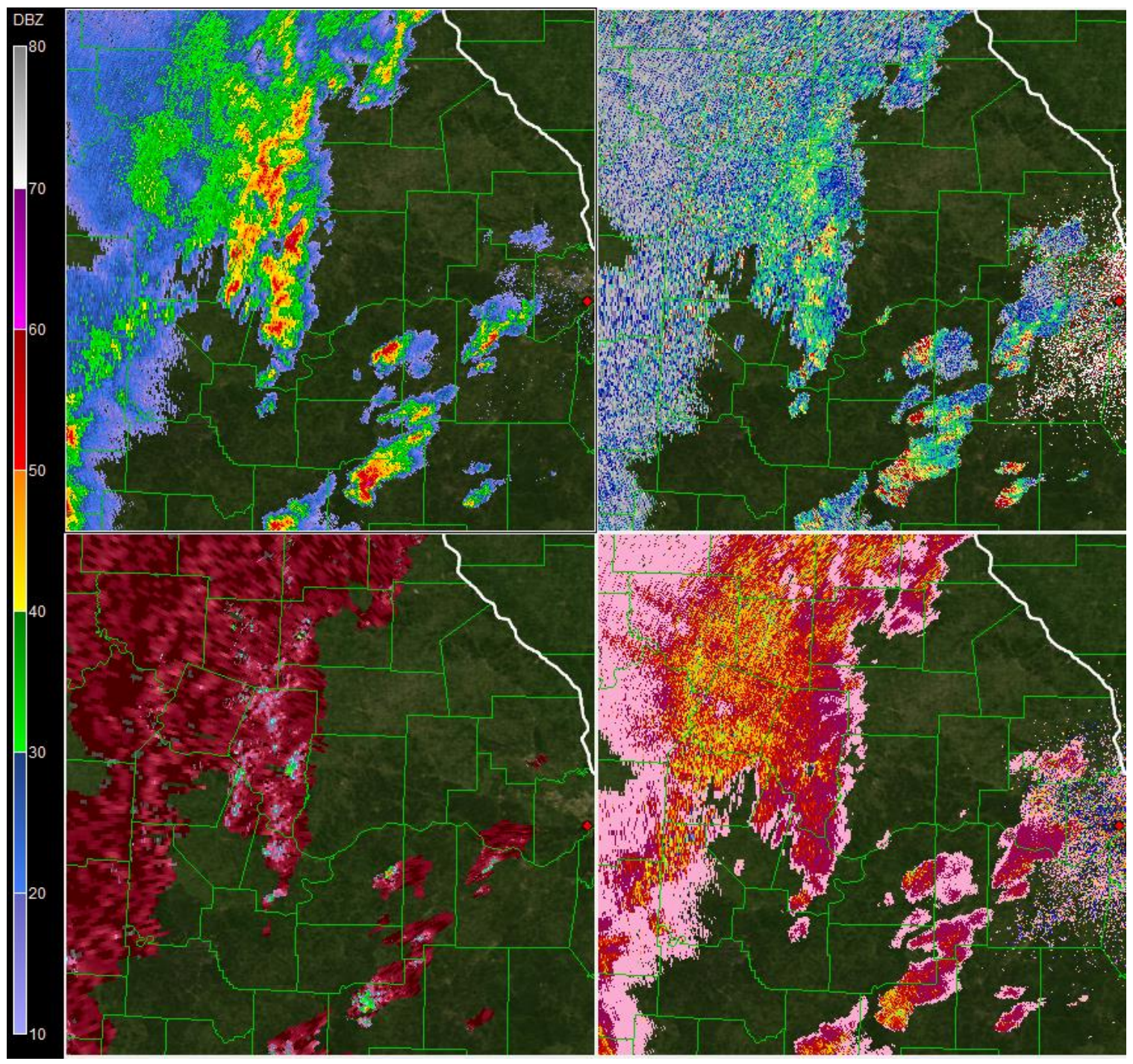

Figure 3.10.4. Four Panel Radar Image for 2154 UTC April 10, 2013. The top left is reflectivity $(Z)$, top right is differential reflectivity $\left(Z_{D R}\right)$, bottom left is specific phase differential $\left(K_{D P}\right)$, and bottom right is correlation coefficient. The scale on the left corresponds to the top left image (Z). The elevation angle used here was $0.5^{\circ}$. 
Table 3.10.1 Conventional Techniques, April 10, 2013

\begin{tabular}{|c|c|c|c|c|c|}
\hline$Z=a R^{b}$ & $\#$ & $\mathrm{FB}(\%)$ & $\mathrm{FAD}(\%)$ & FRMSE(\%) & $\mathrm{FSD}(\%)$ \\
\hline 1 & 59 & -6.02 & 31.72 & 47.56 & 47.18 \\
\hline 2 & 59 & 3.07 & 36.04 & 59.11 & 59.03 \\
\hline 3 & 59 & 96.13 & 114.49 & 205.86 & 182.04 \\
\hline 4 & 59 & -5.38 & 32.95 & 52.21 & 51.93 \\
\hline 5 & 59 & -32.26 & 39.10 & 57.52 & 47.62 \\
\hline \multicolumn{6}{|c|}{$R\left(K_{D P}\right)=a\left|K_{D P}\right|^{b} \operatorname{sign}\left(K_{D P}\right)$} \\
\hline 6 & 61 & 32.54 & 82.19 & 129.99 & 125.85 \\
\hline 7 & 61 & 45.20 & 91.17 & 143.65 & 136.36 \\
\hline 8 & 61 & 45.52 & 90.53 & 138.30 & 130.59 \\
\hline 9 & 61 & 16.68 & 70.97 & 109.81 & 108.54 \\
\hline 10 & 61 & 34.08 & 82.98 & 129.64 & 125.07 \\
\hline 11 & 61 & 27.50 & 78.19 & 120.42 & 117.24 \\
\hline \multicolumn{6}{|c|}{$R\left(Z, Z_{D R}\right)=a Z^{b} Z_{d r}^{c}$} \\
\hline 12 & 59 & -1.79 & 35.75 & 51.62 & 51.59 \\
\hline 13 & 59 & 0.13 & 40.82 & 60.81 & 60.81 \\
\hline 14 & 59 & -23.02 & 33.98 & 49.56 & 43.89 \\
\hline 15 & 59 & -23.25 & 34.11 & 49.27 & 43.44 \\
\hline 16 & 59 & -24.80 & 34.73 & 50.65 & 44.17 \\
\hline \multicolumn{6}{|c|}{$R\left(K_{D P}, Z_{D R}\right)=a\left|K_{D P}\right|^{b} Z_{d r}{ }^{c} \operatorname{sign}\left(K_{D P}\right)$} \\
\hline 17 & 61 & 64.71 & 110.08 & 173.00 & 160.44 \\
\hline 18 & 61 & 97.44 & 143.48 & 232.19 & 210.76 \\
\hline 19 & 61 & 27.00 & 78.02 & 119.19 & 116.09 \\
\hline 20 & 61 & 46.96 & 93.17 & 143.95 & 136.08 \\
\hline \multicolumn{6}{|l|}{ Synthetic } \\
\hline 21 & 61 & 12.86 & 60.77 & 99.45 & 98.61 \\
\hline
\end{tabular}


Table 3.10.2 Trace Techniques, April 10, 2013

\begin{tabular}{|c|c|c|c|c|c|c|}
\hline & $Z_{D R}+$ & $\#$ & $\mathrm{FB}(\%)$ & FAD(\%) & FRMSE(\%) & FSD(\%) \\
\hline \multirow[t]{14}{*}{ T1 } & 0 & 56 & 149.48 & 164.86 & 280.14 & 236.93 \\
\hline & 0.2 & 56 & 124.93 & 141.86 & 246.03 & 211.95 \\
\hline & 0.3 & 56 & 113.94 & 131.53 & 230.79 & 200.71 \\
\hline & 0.4 & 56 & 103.49 & 121.69 & 216.01 & 189.61 \\
\hline & 0.5 & 56 & 93.67 & 112.42 & 202.74 & 179.81 \\
\hline & 0.6 & 55 & 84.47 & 103.98 & 189.31 & 169.42 \\
\hline & 0.7 & 55 & 75.29 & 95.62 & 176.80 & 159.97 \\
\hline & 0.8 & 54 & 66.54 & 88.22 & 163.24 & 149.07 \\
\hline & 0.9 & 54 & 58.46 & 81.97 & 152.39 & 140.73 \\
\hline & 1 & 54 & 50.65 & 75.86 & 141.86 & 132.51 \\
\hline & 1.1 & 54 & 43.81 & 70.58 & 133.26 & 125.85 \\
\hline & 1.5 & 54 & 21.05 & 55.28 & 106.70 & 104.60 \\
\hline & 2 & 54 & -1.53 & 44.50 & 82.90 & 82.89 \\
\hline & 2.6 & 53 & -23.14 & 42.76 & 72.42 & 68.63 \\
\hline \multirow[t]{12}{*}{$\mathrm{T} 2$} & 0.4 & 55 & 114.52 & 126.55 & 196.95 & 160.23 \\
\hline & 0.6 & 55 & 89.70 & 103.80 & 163.20 & 136.34 \\
\hline & 0.8 & 55 & 68.80 & 85.26 & 136.15 & 117.48 \\
\hline & 1 & 55 & 51.77 & 70.90 & 115.92 & 103.72 \\
\hline & 1.2 & 55 & 37.04 & 59.93 & 98.76 & 91.55 \\
\hline & 1.4 & 55 & 25.06 & 52.55 & 87.13 & 83.45 \\
\hline & 1.6 & 55 & 14.37 & 46.75 & 77.41 & 76.07 \\
\hline & 1.8 & 55 & 4.91 & 43.87 & 72.00 & 71.84 \\
\hline & 2 & 55 & -3.22 & 41.74 & 70.48 & 70.41 \\
\hline & 2.2 & 55 & -10.14 & 43.38 & 72.22 & 71.50 \\
\hline & 2.4 & 55 & -15.97 & 46.60 & 76.54 & 74.85 \\
\hline & 2.6 & 55 & -21.47 & 50.56 & 80.72 & 77.81 \\
\hline \multirow[t]{14}{*}{ T3 } & 0 & 58 & 139.73 & 150.00 & 247.34 & 204.08 \\
\hline & 0.2 & 58 & 115.61 & 127.76 & 213.18 & 179.11 \\
\hline & 0.3 & 58 & 104.29 & 117.30 & 197.15 & 167.31 \\
\hline & 0.4 & 58 & 94.01 & 108.46 & 182.85 & 156.83 \\
\hline & 0.5 & 58 & 83.43 & 99.44 & 167.65 & 145.42 \\
\hline & 0.6 & 58 & 73.10 & 90.62 & 153.18 & 134.62 \\
\hline & 0.7 & 57 & 63.71 & 82.59 & 139.18 & 123.75 \\
\hline & 0.8 & 56 & 55.05 & 75.41 & 126.51 & 113.90 \\
\hline & 0.9 & 55 & 47.27 & 69.09 & 115.77 & 105.68 \\
\hline & 1 & 55 & 40.20 & 63.54 & 107.13 & 99.30 \\
\hline & 1.1 & 55 & 33.43 & 58.33 & 98.64 & 92.80 \\
\hline & 1.5 & 55 & 11.66 & 42.95 & 75.43 & 74.52 \\
\hline & 2 & 54 & -9.03 & 35.97 & 64.40 & 63.76 \\
\hline & 2.6 & 54 & -25.77 & 45.00 & 75.01 & 70.44 \\
\hline
\end{tabular}


Table 3.10.2 Trace Techniques, April 10, 2013 (Continued)

\begin{tabular}{|c|c|c|c|c|c|c|}
\hline & $Z_{D R}+$ & $\#$ & $\mathrm{FB}(\%)$ & $\mathrm{FAD}(\%)$ & FRMSE(\%) & $\mathrm{FSD}(\%)$ \\
\hline \multirow[t]{12}{*}{ T4 } & 0.4 & 58 & 114.23 & 126.30 & 204.82 & 170.01 \\
\hline & 0.6 & 58 & 89.45 & 103.77 & 170.60 & 145.28 \\
\hline & 0.8 & 57 & 68.52 & 85.29 & 141.35 & 123.63 \\
\hline & 1 & 55 & 51.43 & 70.60 & 117.84 & 106.02 \\
\hline & 1.2 & 55 & 36.77 & 58.41 & 99.49 & 92.45 \\
\hline & 1.4 & 55 & 24.85 & 49.65 & 86.63 & 82.99 \\
\hline & 1.6 & 55 & 14.18 & 42.30 & 75.67 & 74.33 \\
\hline & 1.8 & 54 & 4.71 & 36.46 & 68.45 & 68.29 \\
\hline & 2 & 54 & -3.40 & 35.60 & 66.05 & 65.96 \\
\hline & 2.2 & 54 & -10.30 & 36.59 & 67.19 & 66.40 \\
\hline & 2.4 & 54 & -16.12 & 38.87 & 71.26 & 69.42 \\
\hline & 2.6 & 54 & -21.61 & 42.86 & 75.46 & 72.29 \\
\hline \multirow[t]{12}{*}{ T5 } & 0.4 & 56 & 123.93 & 138.30 & 239.19 & 204.59 \\
\hline & 0.6 & 56 & 101.40 & 116.72 & 210.30 & 184.24 \\
\hline & 0.8 & 55 & 80.62 & 97.66 & 180.28 & 161.25 \\
\hline & 1 & 54 & 62.44 & 81.84 & 154.17 & 140.96 \\
\hline & 1.2 & 54 & 47.71 & 70.02 & 135.90 & 127.25 \\
\hline & 1.4 & 54 & 34.98 & 60.28 & 120.44 & 115.25 \\
\hline & 1.6 & 54 & 23.62 & 52.65 & 106.31 & 103.65 \\
\hline & 1.8 & 54 & 13.45 & 46.77 & 94.27 & 93.31 \\
\hline & 2 & 54 & 4.61 & 43.97 & 85.86 & 85.74 \\
\hline & 2.2 & 54 & -3.64 & 42.16 & 79.89 & 79.81 \\
\hline & 2.4 & 53 & -11.41 & 40.72 & 74.90 & 74.02 \\
\hline & 2.6 & 53 & -18.75 & 41.19 & 72.09 & 69.61 \\
\hline
\end{tabular}




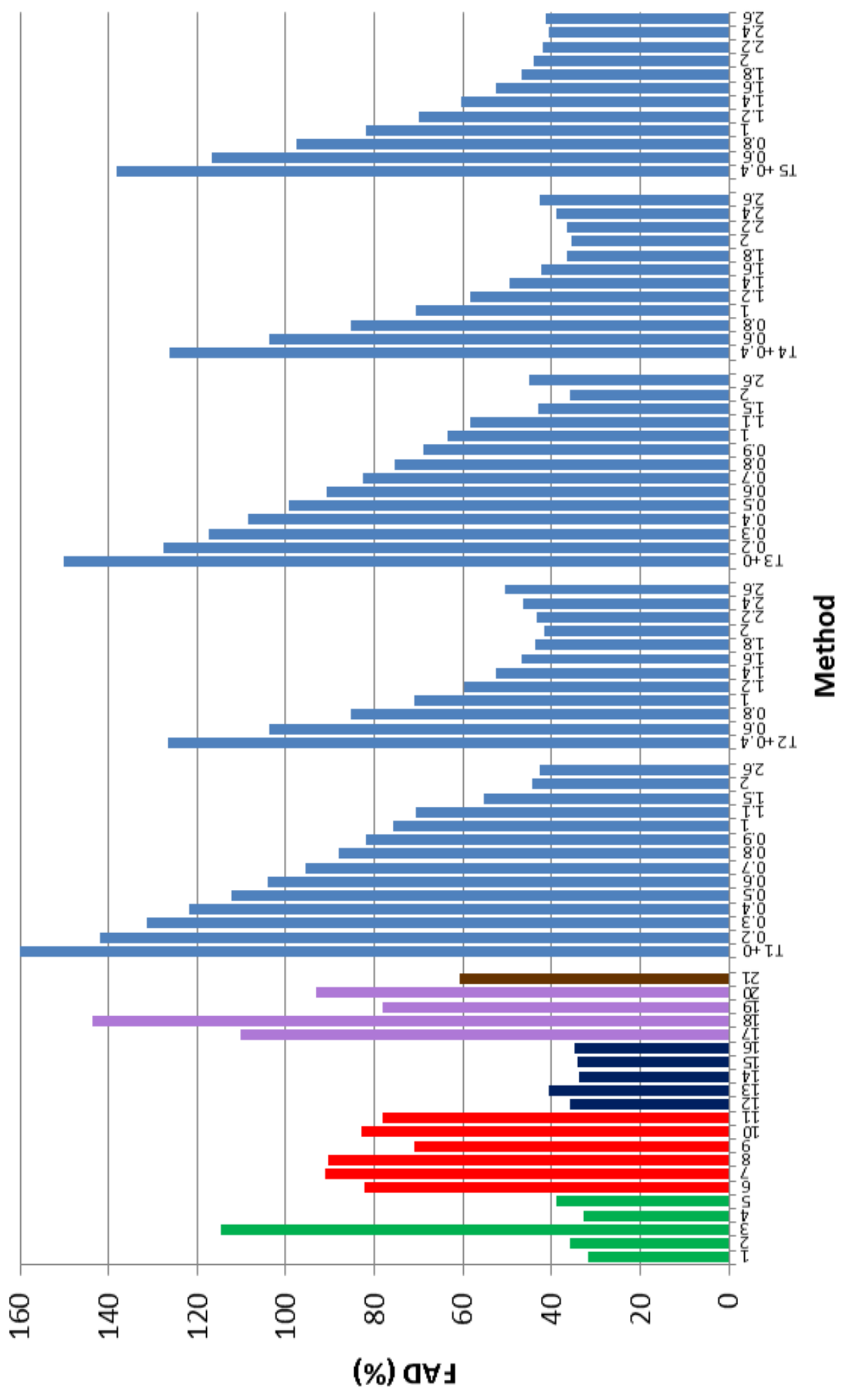

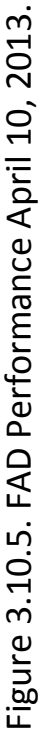




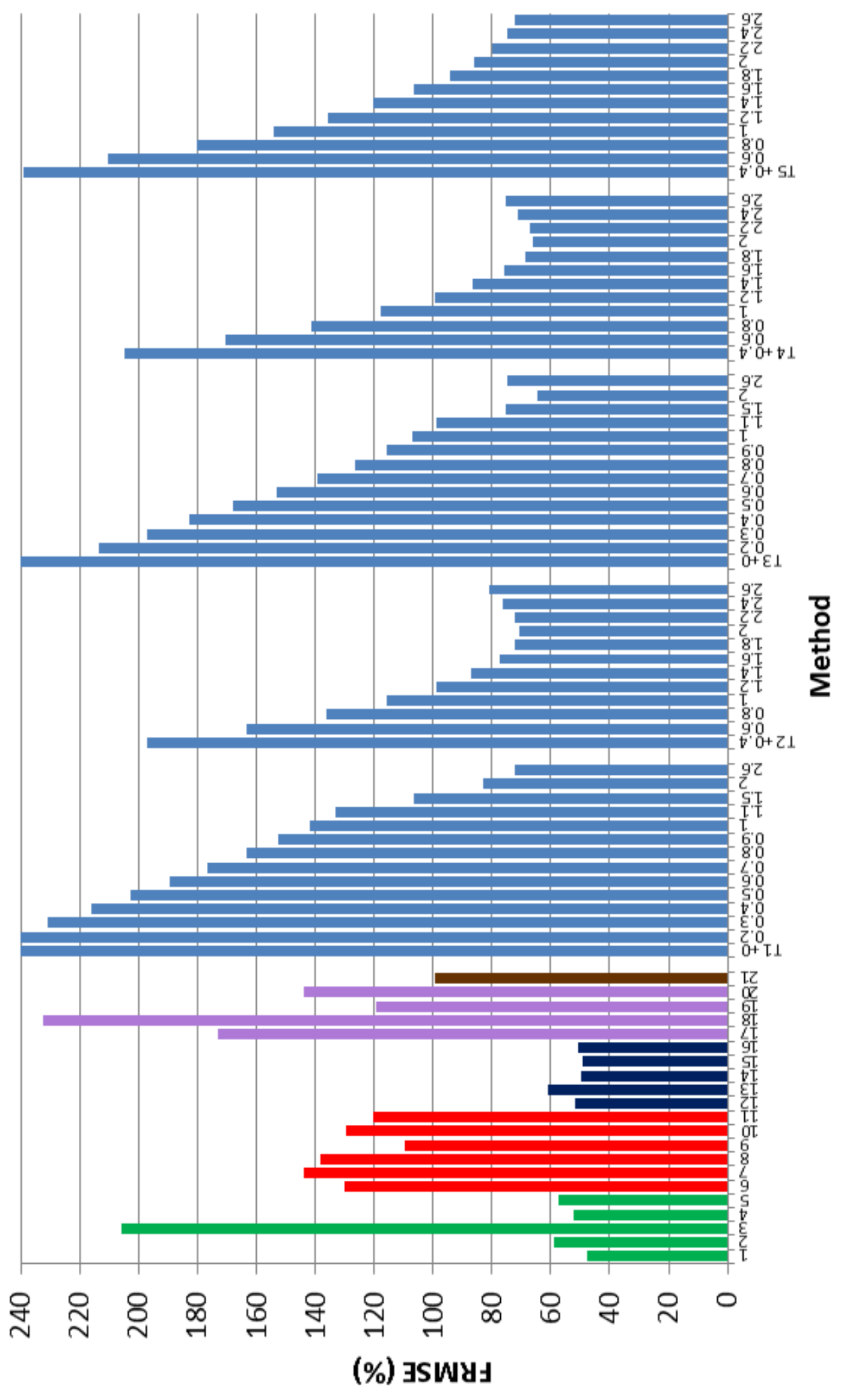

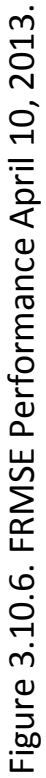




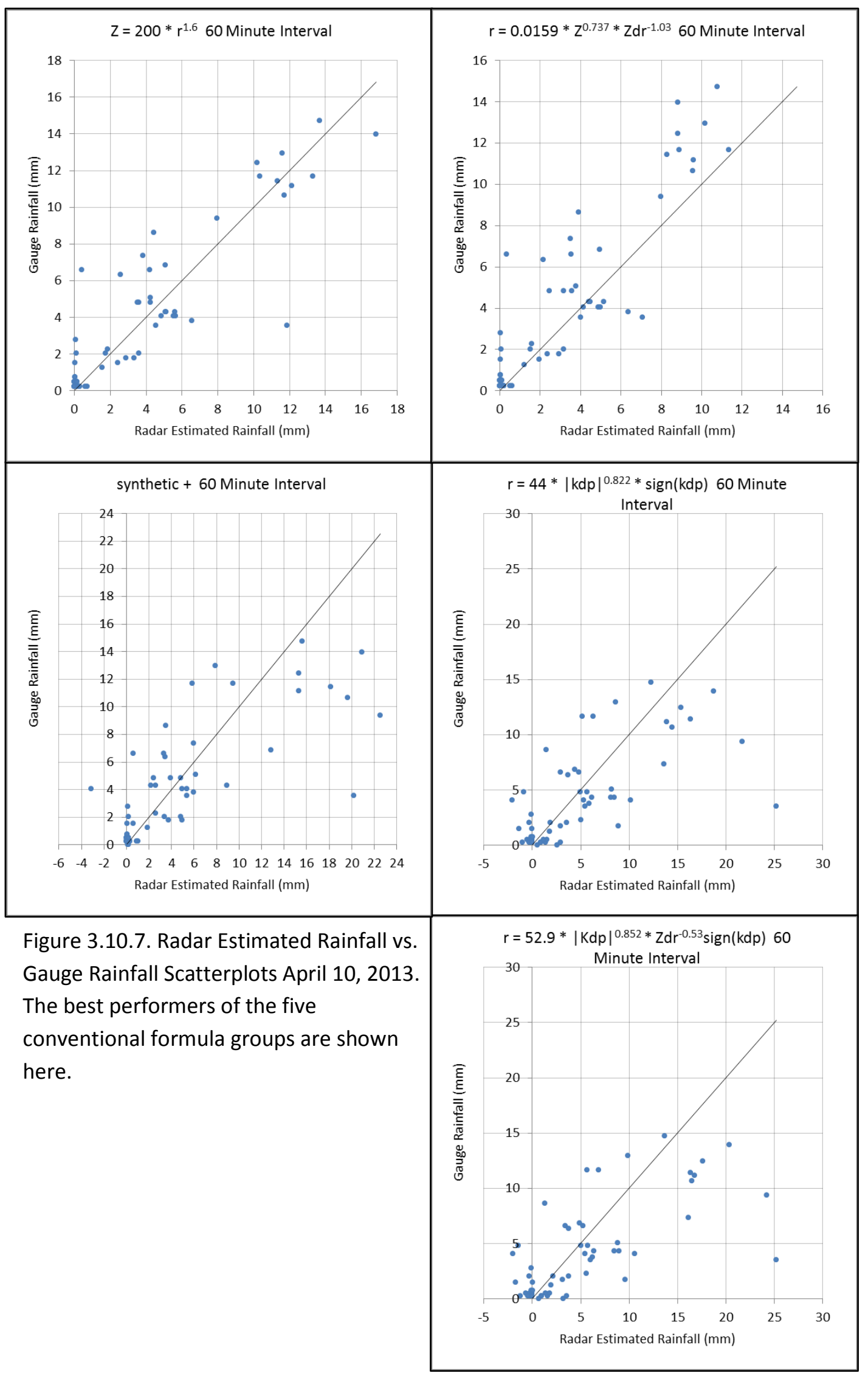



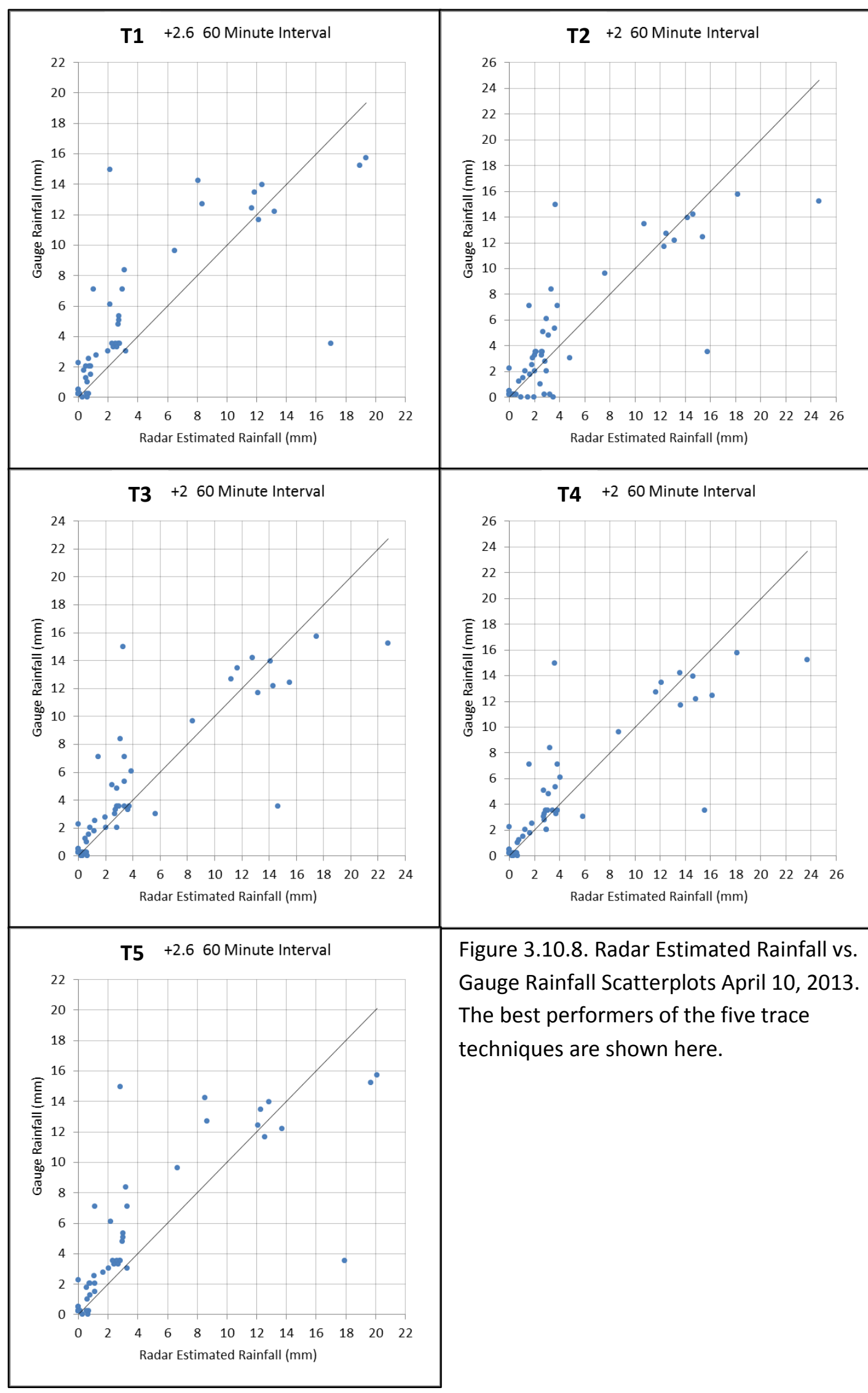

Figure 3.10.8. Radar Estimated Rainfall vs. Gauge Rainfall Scatterplots April 10, 2013. The best performers of the five trace techniques are shown here. 


\subsection{April 17, 2013}

A second hail event occurred on the $17^{\text {th }}$ of April. Up to baseball size hail was reported at locations around Central Missouri. This rainfall event took place along the stationary front located to the east of a low pressure center. Figure 3.11.1 is an HPC surface analysis for 00 UTC of April 18. Figure 3.11 .2 shows humidity versus height in percentile format for 19 UTC of April 17 through 11 UTC of April 18. The same pattern seen earlier of a moistening atmosphere was present for this event. Figure 3.11.3 is a hodograph for the $10^{\text {th }}$ of April. The hodograph shows the wind direction transitioning from southeasterly at the surface to southwesterly aloft. Figure 3.11 .4 is a four panel radar image from KLSX for 0743 UTC of April 18.

\subsubsection{Conventional Formulas}

Formulas involving $\mathrm{K}_{\mathrm{DP}}$ were not evaluated for the April 17 event due to lack of data.

Table 3.11.1 shows the performance of the conventional techniques for this event. The presence of large hail again caused poor performance for all conventional formulas. Mostly positive biases were present, and (3) was again an outlier.

\subsubsection{Tracing Techniques}

Table 3.11.2 shows the performance of the various trace techniques and their permutations for this event. Many of the same patterns from the hail event on September 7, 2012, were also present on this date. The optimal $Z_{D R}$ adjustment for each of the five tracing techniques lay in the 1- 1.5 range, and the optimal T1 technique again performed the best. The optimal T1 and T3 techniques significantly outperformed all 
other methods on this date. Biases for the tracing techniques were mostly positive for this event.

\subsubsection{Graphical Evaluation}

Figure 3.11.5 is a histogram showing the FAD performance of each evaluated technique for this event. Figure 3.11.6 is the equivalent histogram showing FRMSE performance.

Figure 3.11.7 shows a combination of scatterplots of radar-derived rainfall versus rain gauge rainfall for the conventional formulas. The techniques illustrated were the top performers in each category. Figure 3.11.8 is identical to 3.11.7, except it depicts the best performers of the trace techniques. 


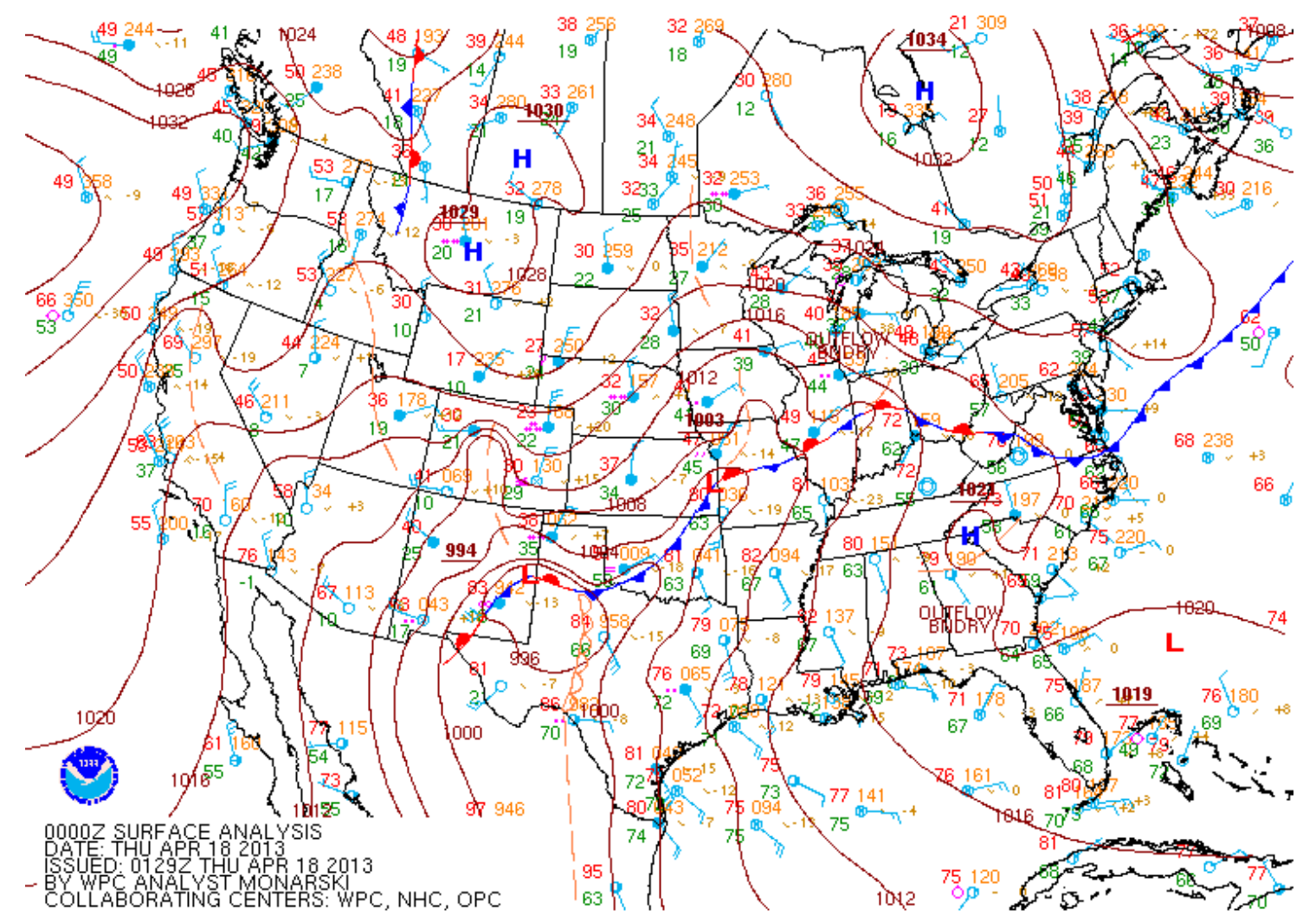

Figure 3.11.1. Surface Analysis 0000 UTC April 18, 2013.

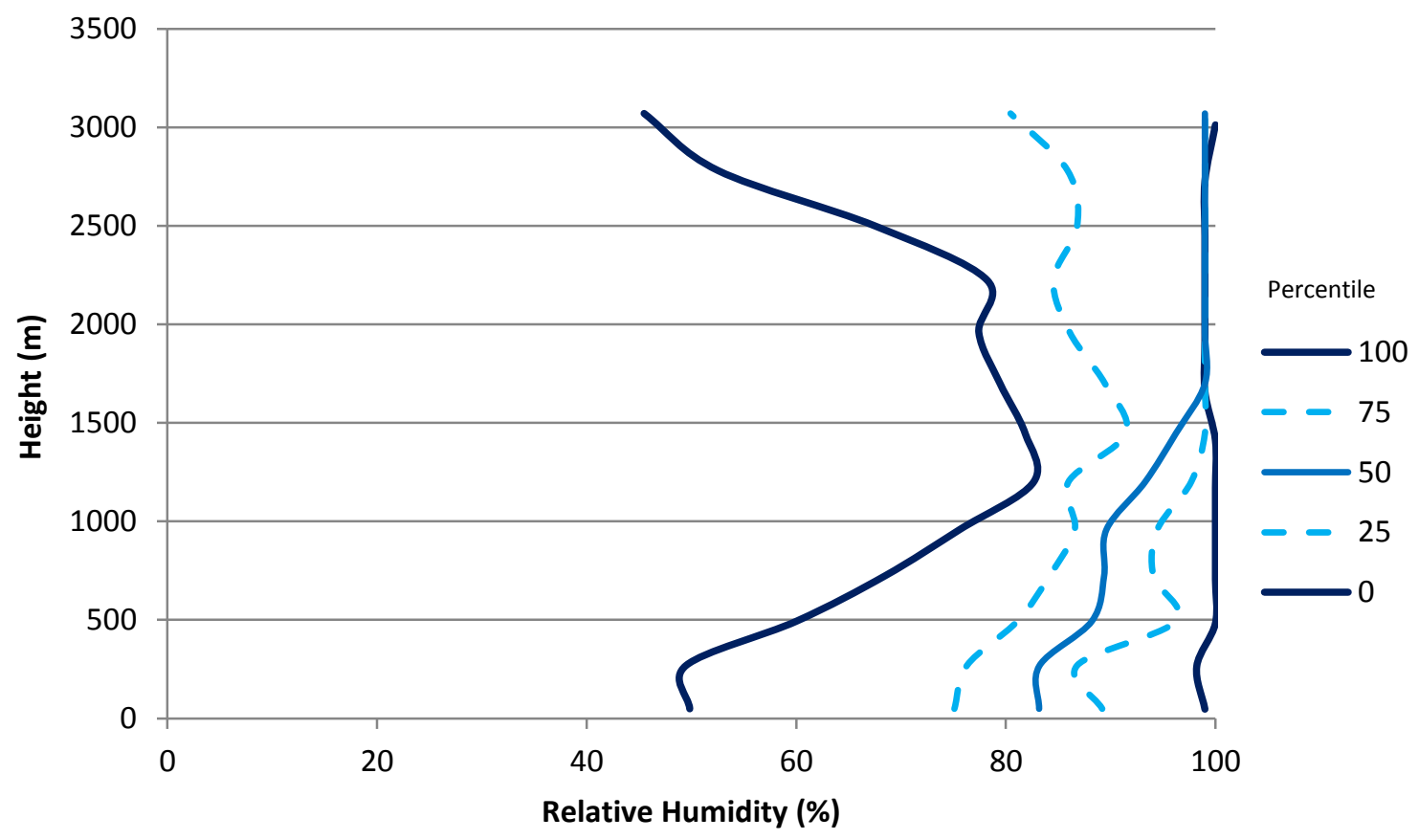

Figure 3.11.2. Relative Humidity Profile April 17, 2013. 


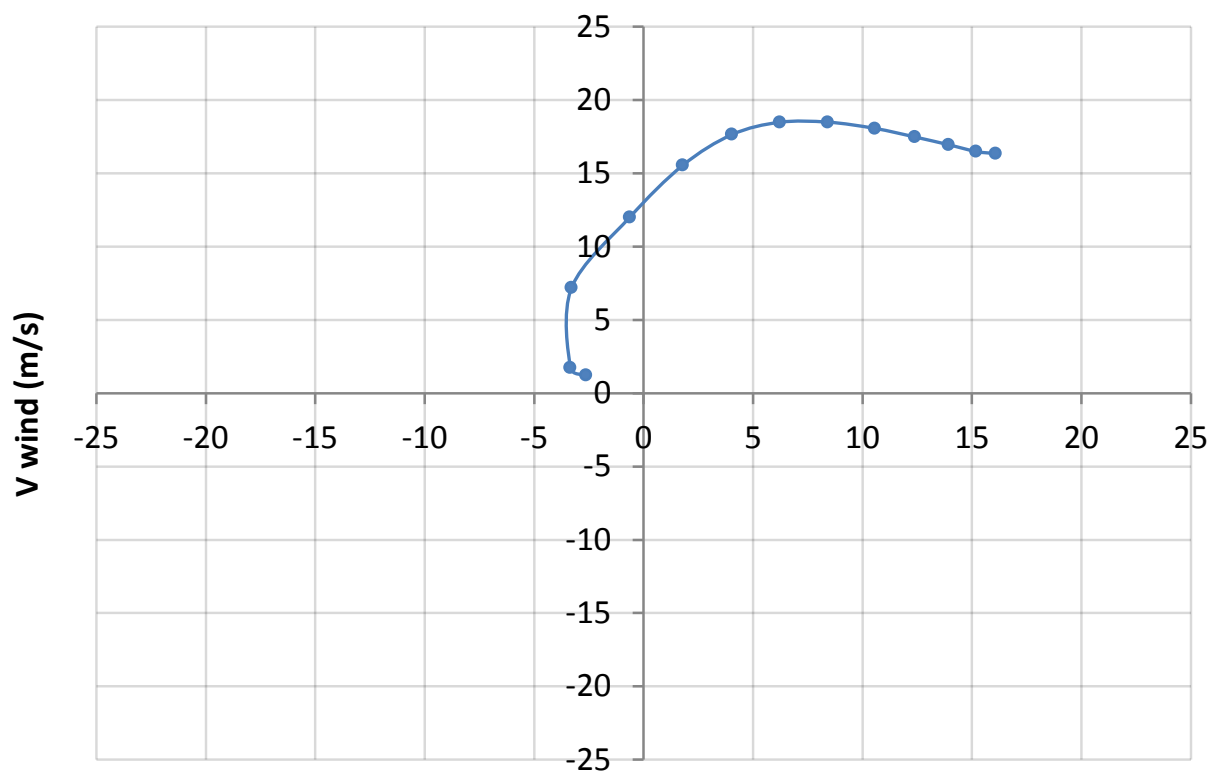

$U$ wind $(\mathrm{m} / \mathrm{s})$

$\longrightarrow$ Mean Wind

Figure 3.11.3. Hodograph April 17, 2013. 


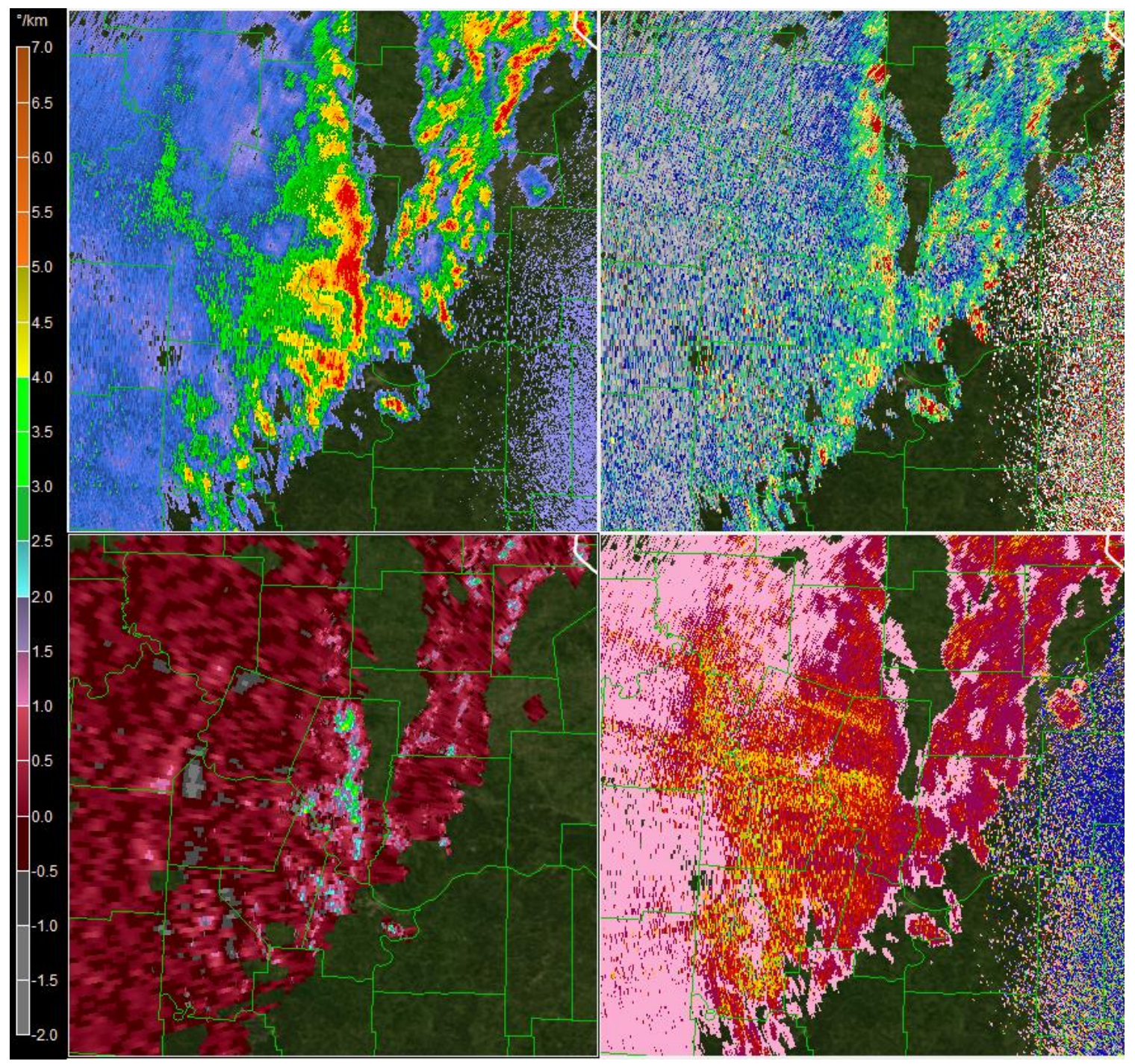

Figure 3.11.4. Four Panel Radar Image for 0743 UTC April 18, 2013. The top left is reflectivity $(Z)$, top right is differential reflectivity $\left(Z_{D R}\right)$, bottom left is specific phase differential $\left(K_{D P}\right)$, and bottom right is correlation coefficient. The scale on the left corresponds to the bottom left image $\left(\mathrm{K}_{\mathrm{DP}}\right)$. The elevation angle used here was $0.5^{\circ}$. 
Table 3.11.1 Conventional Techniques, April 17, 2013

\begin{tabular}{lccccc}
\hline$Z=a R^{b}$ & $\#$ & $\mathrm{FB}(\%)$ & $\mathrm{FAD}(\%)$ & $\mathrm{FRMSE}(\%)$ & $\mathrm{FSD}(\%)$ \\
\hline 1 & 83 & 13.61 & 48.69 & 79.70 & 78.53 \\
2 & 79 & 30.64 & 61.91 & 104.96 & 100.38 \\
3 & 79 & 169.17 & 181.25 & 336.78 & 291.21 \\
4 & 79 & 18.52 & 54.96 & 90.14 & 88.22 \\
5 & 75 & -14.57 & 50.37 & 73.72 & 72.27 \\
\hline$R\left(Z, Z_{D R}\right)=a Z^{b} Z_{d r}{ }^{c}$ & & & & & \\
\hline 12 & 73 & -29.48 & 54.70 & 82.33 & 76.87 \\
13 & 71 & -37.19 & 61.82 & 93.78 & 86.09 \\
14 & 76 & -33.15 & 52.32 & 80.61 & 73.47 \\
15 & 77 & -29.05 & 50.20 & 76.19 & 70.44 \\
16 & 76 & -33.66 & 52.57 & 80.48 & 73.10 \\
\hline
\end{tabular}


Table 3.11.2 Trace Techniques, April 17, 2013

\begin{tabular}{|c|c|c|c|c|c|c|}
\hline & $Z_{D R}+$ & $\#$ & $\mathrm{FB}(\%)$ & FAD(\%) & FRMSE(\%) & FSD(\%) \\
\hline \multirow[t]{14}{*}{$\mathrm{T} 1$} & 0 & 71 & 54.10 & 71.83 & 107.80 & 93.24 \\
\hline & 0.2 & 71 & 43.13 & 61.46 & 89.07 & 77.94 \\
\hline & 0.3 & 70 & 38.40 & 57.04 & 81.07 & 71.40 \\
\hline & 0.4 & 70 & 34.50 & 53.21 & 75.48 & 67.13 \\
\hline & 0.5 & 69 & 31.27 & 49.94 & 71.02 & 63.76 \\
\hline & 0.6 & 69 & 28.56 & 47.00 & 68.16 & 61.89 \\
\hline & 0.7 & 69 & 25.68 & 43.97 & 64.93 & 59.63 \\
\hline & 0.8 & 69 & 22.72 & 41.48 & 61.44 & 57.09 \\
\hline & 0.9 & 69 & 20.04 & 39.20 & 58.48 & 54.94 \\
\hline & 1 & 69 & 17.63 & 37.07 & 56.32 & 53.49 \\
\hline & 1.1 & 70 & 15.68 & 36.61 & 55.75 & 53.50 \\
\hline & 1.5 & 70 & 9.72 & 40.15 & 60.25 & 59.46 \\
\hline & 2 & 69 & 0.71 & 44.78 & 67.77 & 67.77 \\
\hline & 2.6 & 69 & -9.97 & 51.31 & 77.35 & 76.71 \\
\hline \multirow[t]{12}{*}{$\mathrm{T} 2$} & 0.4 & 78 & 74.70 & 82.45 & 129.43 & 105.70 \\
\hline & 0.6 & 78 & 62.06 & 72.20 & 114.09 & 95.74 \\
\hline & 0.8 & 77 & 52.26 & 64.68 & 102.70 & 88.41 \\
\hline & 1 & 75 & 45.04 & 59.02 & 94.93 & 83.57 \\
\hline & 1.2 & 74 & 39.37 & 55.20 & 91.00 & 82.04 \\
\hline & 1.4 & 72 & 34.05 & 52.64 & 87.67 & 80.79 \\
\hline & 1.6 & 71 & 29.88 & 52.98 & 88.77 & 83.60 \\
\hline & 1.8 & 70 & 27.32 & 55.71 & 94.63 & 90.60 \\
\hline & 2 & 70 & 26.04 & 60.40 & 105.15 & 101.88 \\
\hline & 2.2 & 70 & 24.86 & 64.73 & 115.83 & 113.13 \\
\hline & 2.4 & 70 & 22.50 & 67.92 & 123.09 & 121.01 \\
\hline & 2.6 & 70 & 19.82 & 71.74 & 128.55 & 127.02 \\
\hline \multirow[t]{14}{*}{ T3 } & 0 & 75 & 66.02 & 85.86 & 129.85 & 111.82 \\
\hline & 0.2 & 75 & 53.18 & 75.45 & 113.18 & 99.91 \\
\hline & 0.3 & 75 & 47.40 & 70.67 & 105.93 & 94.73 \\
\hline & 0.4 & 75 & 42.24 & 66.46 & 99.68 & 90.29 \\
\hline & 0.5 & 73 & 37.49 & 62.69 & 93.28 & 85.42 \\
\hline & 0.6 & 73 & 33.20 & 59.08 & 88.73 & 82.28 \\
\hline & 0.7 & 73 & 29.30 & 55.92 & 84.59 & 79.36 \\
\hline & 0.8 & 72 & 25.98 & 53.12 & 80.95 & 76.67 \\
\hline & 0.9 & 72 & 23.09 & 50.47 & 78.11 & 74.62 \\
\hline & 1 & 72 & 20.53 & 48.33 & 75.93 & 73.11 \\
\hline & 1.1 & 72 & 18.23 & 46.48 & 74.14 & 71.86 \\
\hline & 1.5 & 71 & 9.24 & 42.37 & 66.76 & 66.12 \\
\hline & 2 & 70 & 3.07 & 47.59 & 73.14 & 73.08 \\
\hline & 2.6 & 69 & -3.34 & 56.94 & 88.37 & 88.31 \\
\hline
\end{tabular}


Table 3.11.2 Trace Techniques, April 17, 2013 (Continued)

\begin{tabular}{|c|c|c|c|c|c|c|}
\hline & $Z_{D R}+$ & $\#$ & $\mathrm{FB}(\%)$ & FAD(\%) & FRMSE(\%) & FSD(\%) \\
\hline \multirow[t]{12}{*}{ T4 } & 0.4 & 75 & 66.51 & 74.78 & 116.02 & 95.06 \\
\hline & 0.6 & 75 & 55.21 & 66.16 & 104.43 & 88.65 \\
\hline & 0.8 & 74 & 46.49 & 59.92 & 95.80 & 83.76 \\
\hline & 1 & 72 & 40.13 & 55.33 & 89.93 & 80.49 \\
\hline & 1.2 & 72 & 35.17 & 52.88 & 87.94 & 80.60 \\
\hline & 1.4 & 71 & 30.40 & 51.87 & 86.10 & 80.55 \\
\hline & 1.6 & 71 & 26.67 & 53.69 & 88.33 & 84.20 \\
\hline & 1.8 & 71 & 24.47 & 57.23 & 95.04 & 91.84 \\
\hline & 2 & 71 & 23.45 & 61.79 & 105.58 & 102.95 \\
\hline & 2.2 & 70 & 22.45 & 65.91 & 115.44 & 113.23 \\
\hline & 2.4 & 70 & 20.31 & 69.06 & 122.75 & 121.06 \\
\hline & 2.6 & 69 & 17.79 & 72.96 & 127.33 & 126.09 \\
\hline \multirow[t]{12}{*}{ T5 } & 0.4 & 71 & 58.07 & 66.86 & 94.53 & 74.59 \\
\hline & 0.6 & 71 & 50.37 & 60.99 & 88.33 & 72.55 \\
\hline & 0.8 & 70 & 43.48 & 56.25 & 83.16 & 70.89 \\
\hline & 1 & 70 & 37.92 & 53.01 & 82.05 & 72.76 \\
\hline & 1.2 & 70 & 34.39 & 52.81 & 86.73 & 79.62 \\
\hline & 1.4 & 70 & 31.69 & 54.92 & 93.88 & 88.37 \\
\hline & 1.6 & 70 & 29.13 & 58.24 & 102.16 & 97.92 \\
\hline & 1.8 & 70 & 25.69 & 60.56 & 107.79 & 104.69 \\
\hline & 2 & 70 & 21.42 & 62.07 & 110.83 & 108.74 \\
\hline & 2.2 & 70 & 17.38 & 63.25 & 114.03 & 112.70 \\
\hline & 2.4 & 69 & 13.17 & 64.27 & 115.59 & 114.83 \\
\hline & 2.6 & 69 & 9.37 & 66.26 & 118.15 & 117.78 \\
\hline
\end{tabular}




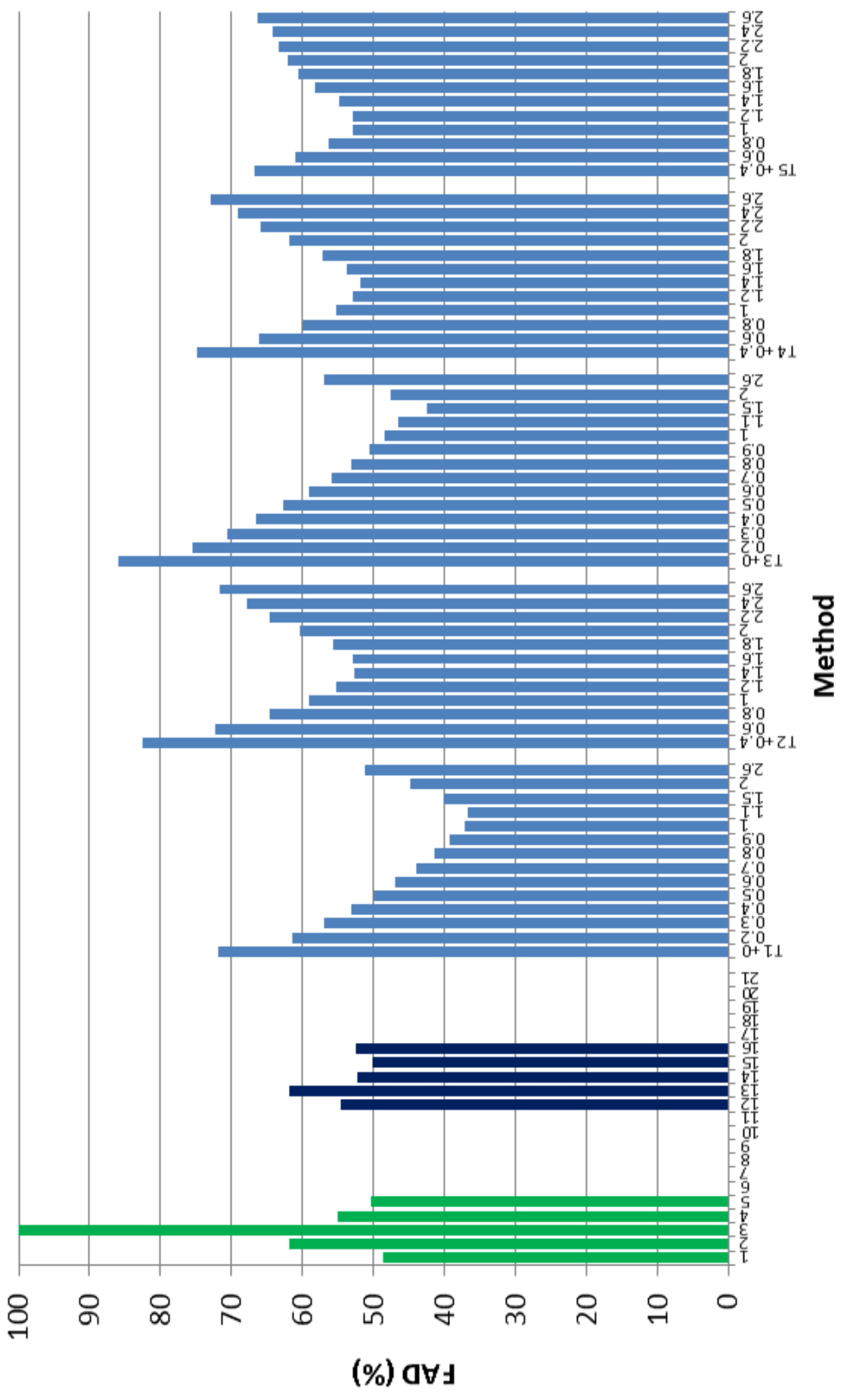

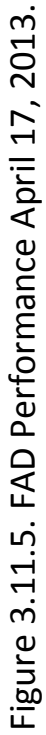




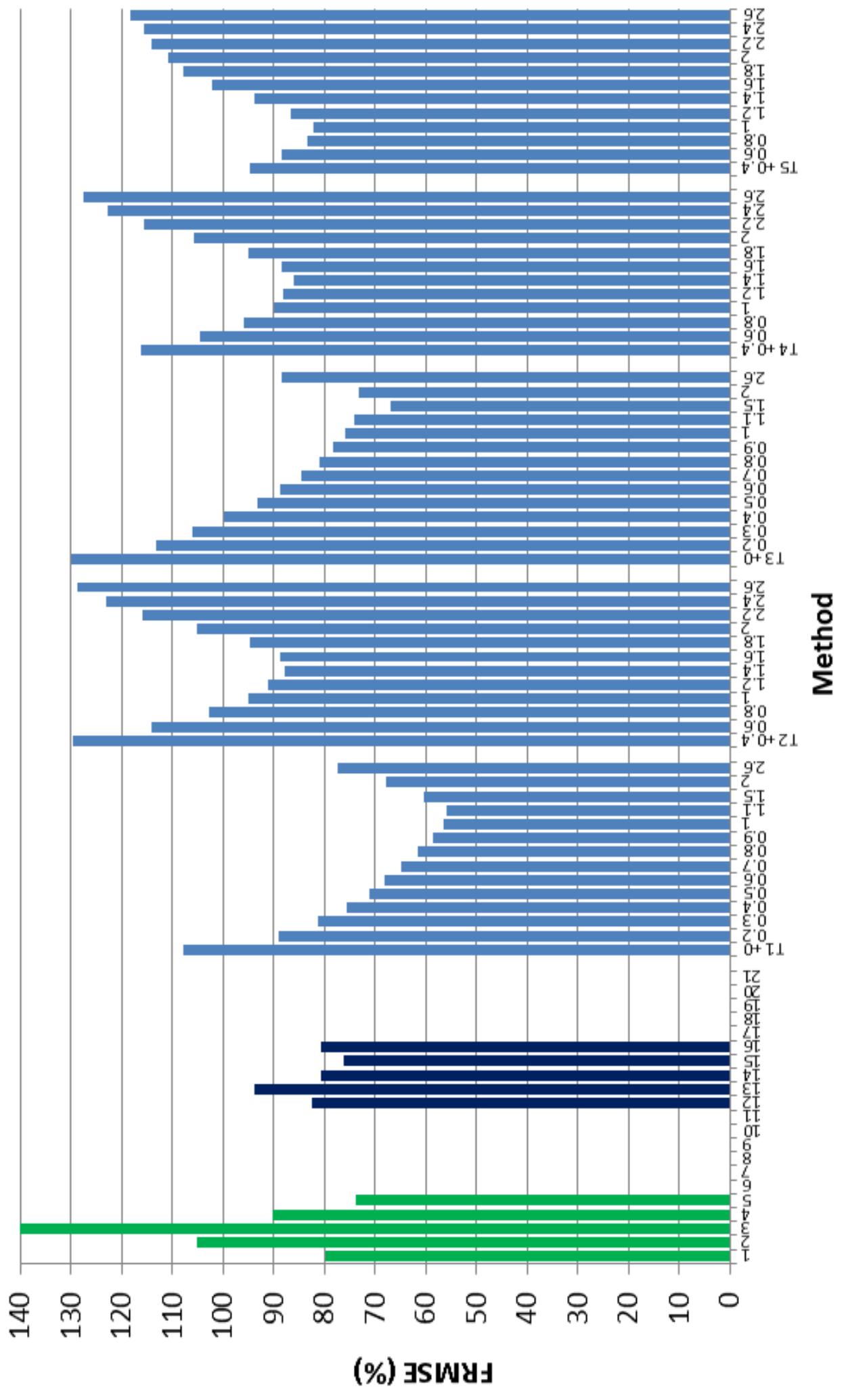

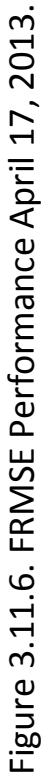




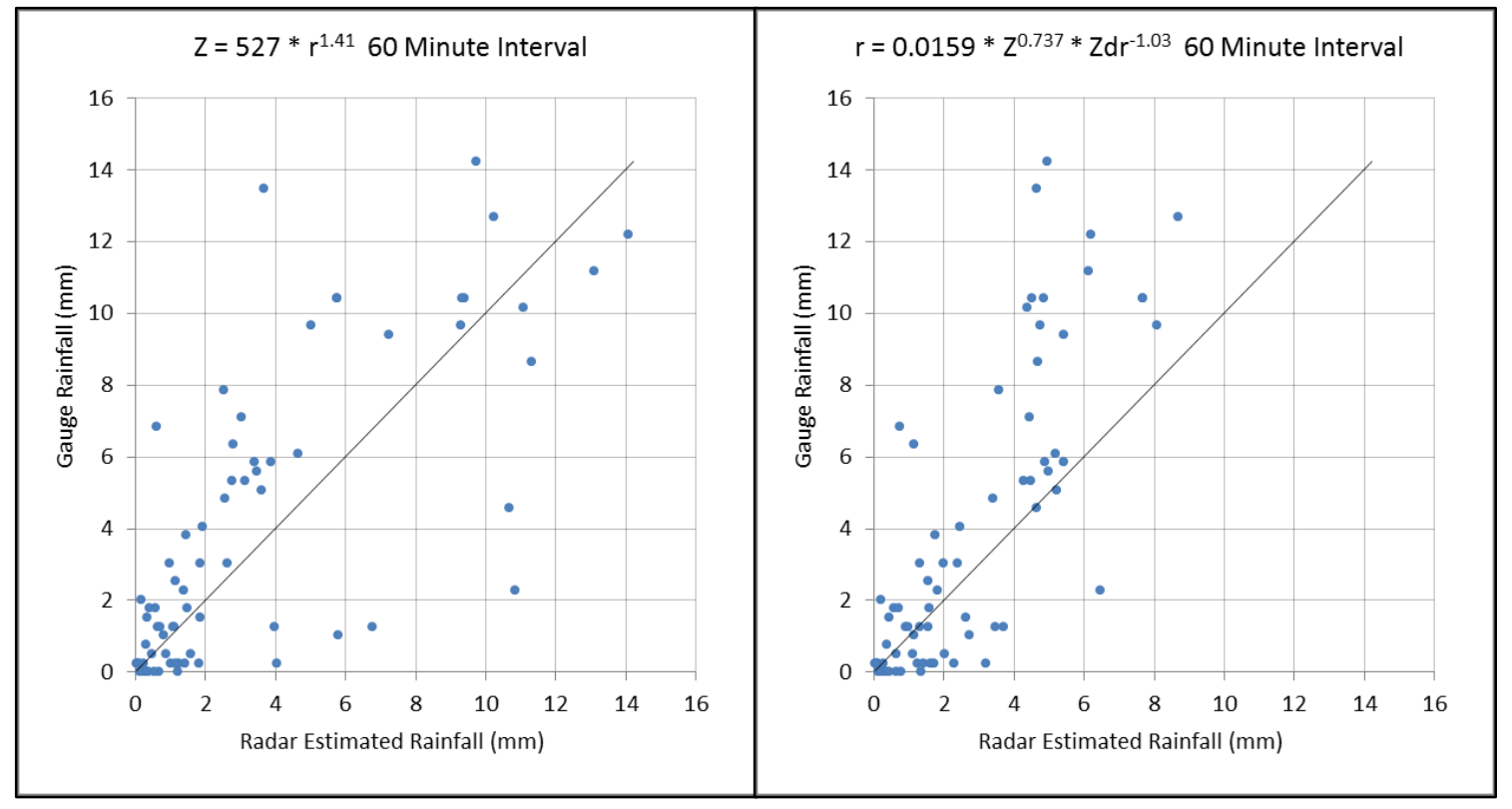

Figure 3.11.7. Radar Estimated Rainfall vs. Gauge Rainfall Scatterplots April 17, 2013. The best performers of the five conventional formula groups are shown here. 

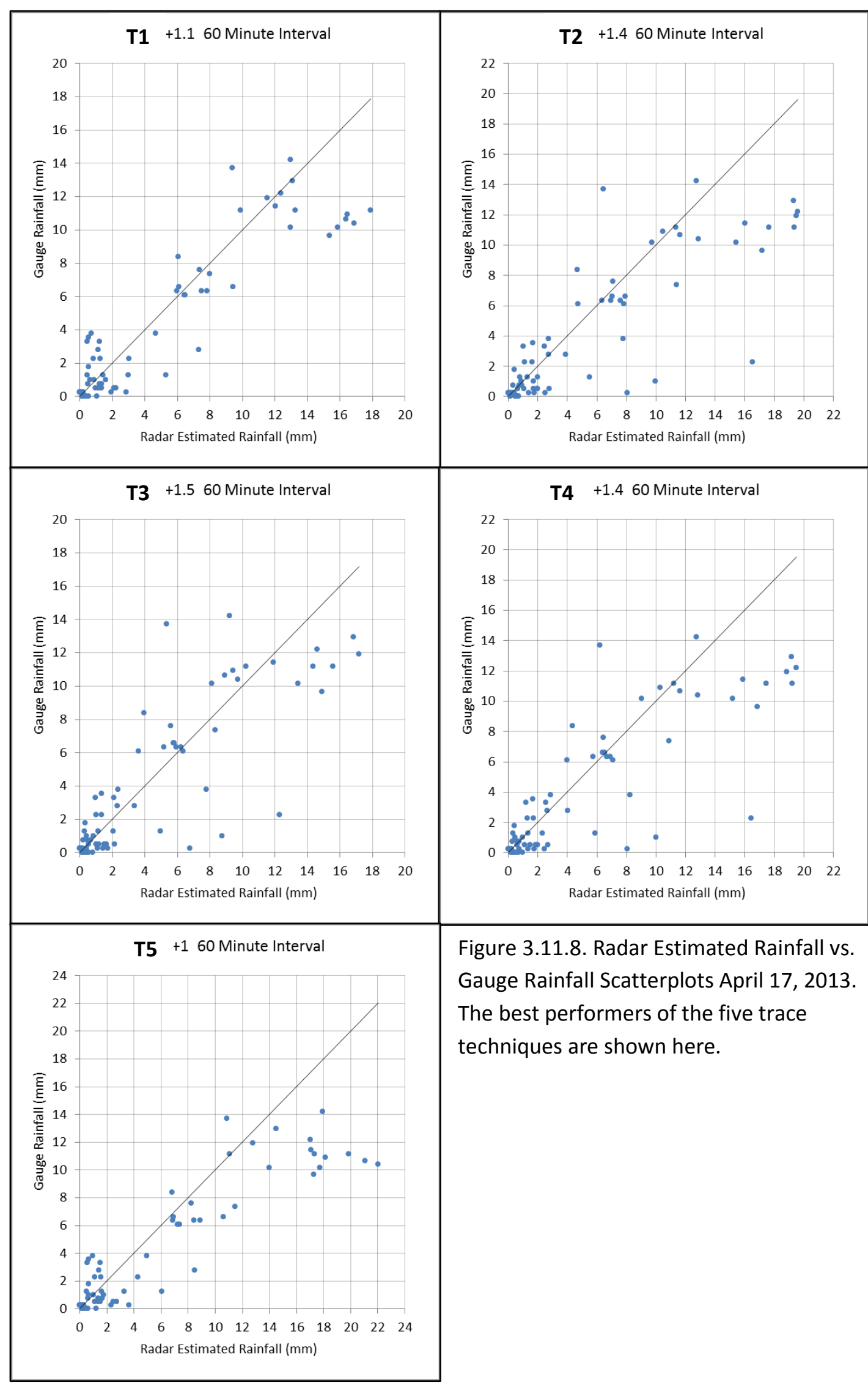

Figure 3.11.8. Radar Estimated Rainfall vs. Gauge Rainfall Scatterplots April 17, 2013.

The best performers of the five trace techniques are shown here. 


\subsection{May 30, 2013}

The May 30 rainfall event was both the longest and heaviest of the studied events. More than 100 millimeters of rain fell over a two day period in several locations around Central Missouri. The rain with this event was associated with two slow moving centers of low pressure far to the west and northwest. Figure 3.12.1 is an HPC surface analysis for 21 UTC of May 30. Figure 3.12.2 shows humidity versus height in percentile format for 08 UTC of May 30 through 18 UTC of May 31. Due to the extended nature of the event multiple drying and moistening periods were present, resulting in a very wide range of relative humidity values (especially at higher levels). Figure 3.12 .3 is a hodograph for the $30^{\text {th }}$ of May. The hodograph shows the wind direction transitioning from southerly at the surface to southwesterly aloft. Figure 3.12.4 is a four panel radar image from KLSX for 2044 UTC of May 30. Several waves of storms passed through Central Missouri over the period of this event.

\subsubsection{Conventional Formulas}

Table 3.12.1 shows the performance of the conventional formulas for this event. The most basic Z-R relationship, (1), was again the top performer of the conventional formulas. Of the non- $K_{D P}$ reliant formulas, one outlier was present, (3). The $K_{D P}$-reliant formulas again performed poorly, though not as poorly as they did on other events. A mix of positive and negative biases was present for the conventional formulas for this event. 


\subsubsection{Tracing Techniques}

Table 3.12.2 shows the performance of the various trace techniques and their permutations for this event. Techniques T2, T4, and T5 were most effective at the $+Z_{D R}$ values of around 2.6 , T1 was most effective at $+Z_{D R}$ values near 0.2 , and $T 3$ was most effective at $a+Z_{D R}$ value of 2 (by $F A D$ and $F R M S E$, $F B$ was much worse at $Z_{D R}+2$ ). However, variation in the FAD and FRMSE evaluation parameters at all $Z_{D R}$ adjustments for the T1 and T3 techniques was minimal. The tracing techniques as a whole performed slightly worse than the non- $K_{D P}$ reliant conventional formulas for this event.

\subsubsection{Graphical Evaluation}

Figure 3.12 .5 is a histogram showing the FAD performance of each evaluated technique for this event. Figure 3.12.6 is the equivalent histogram showing FRMSE performance.

Figure 3.12.7 shows a combination of scatterplots of radar-derived rainfall versus rain gauge rainfall for the conventional formulas. The techniques illustrated were the top performers in each category. Figure 3.12.8 is identical to 3.12.7, except it depicts the best performers of the trace techniques. 


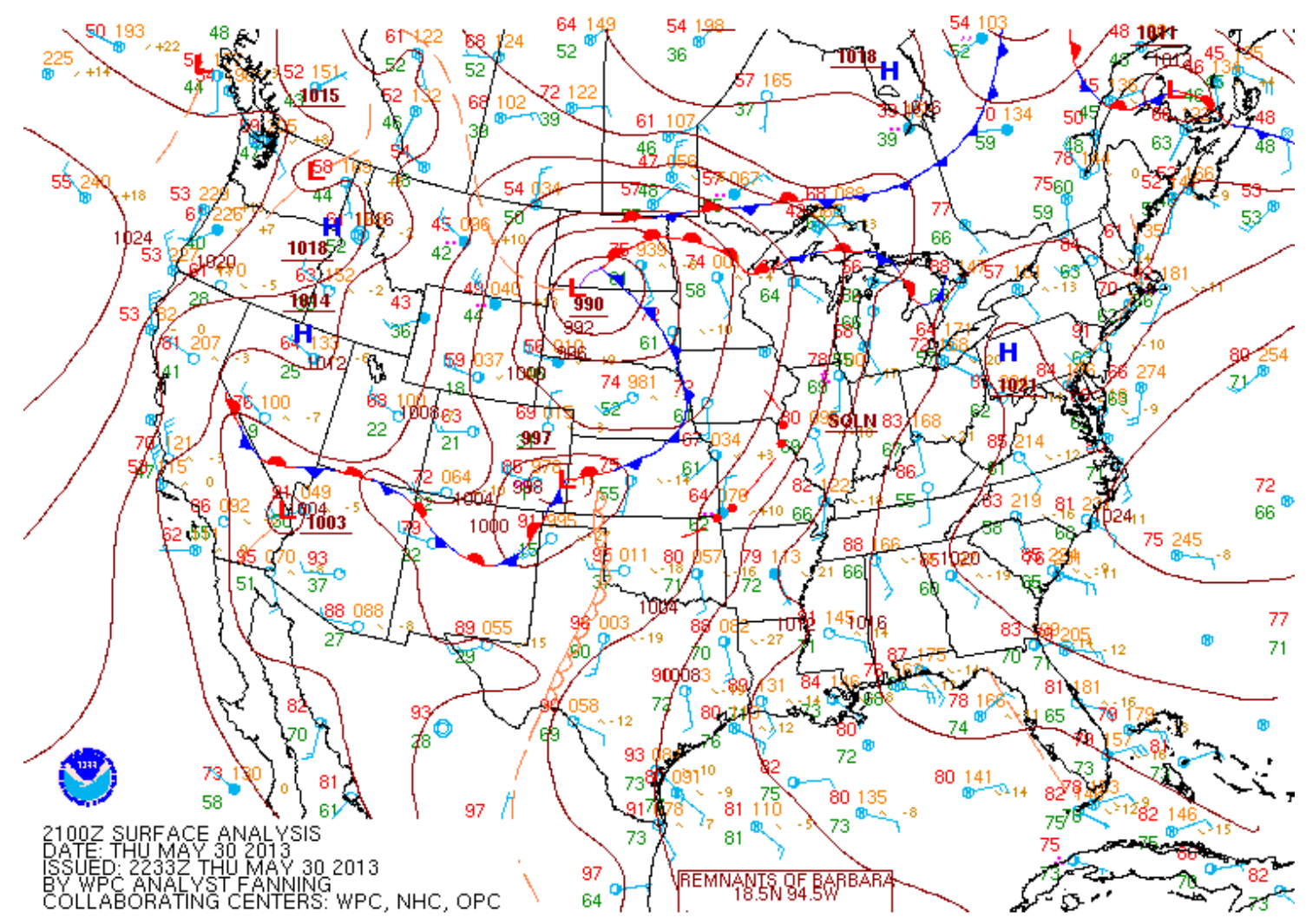

Figure 3.12.1. Surface Analysis 2100 UTC May 30, 2013.

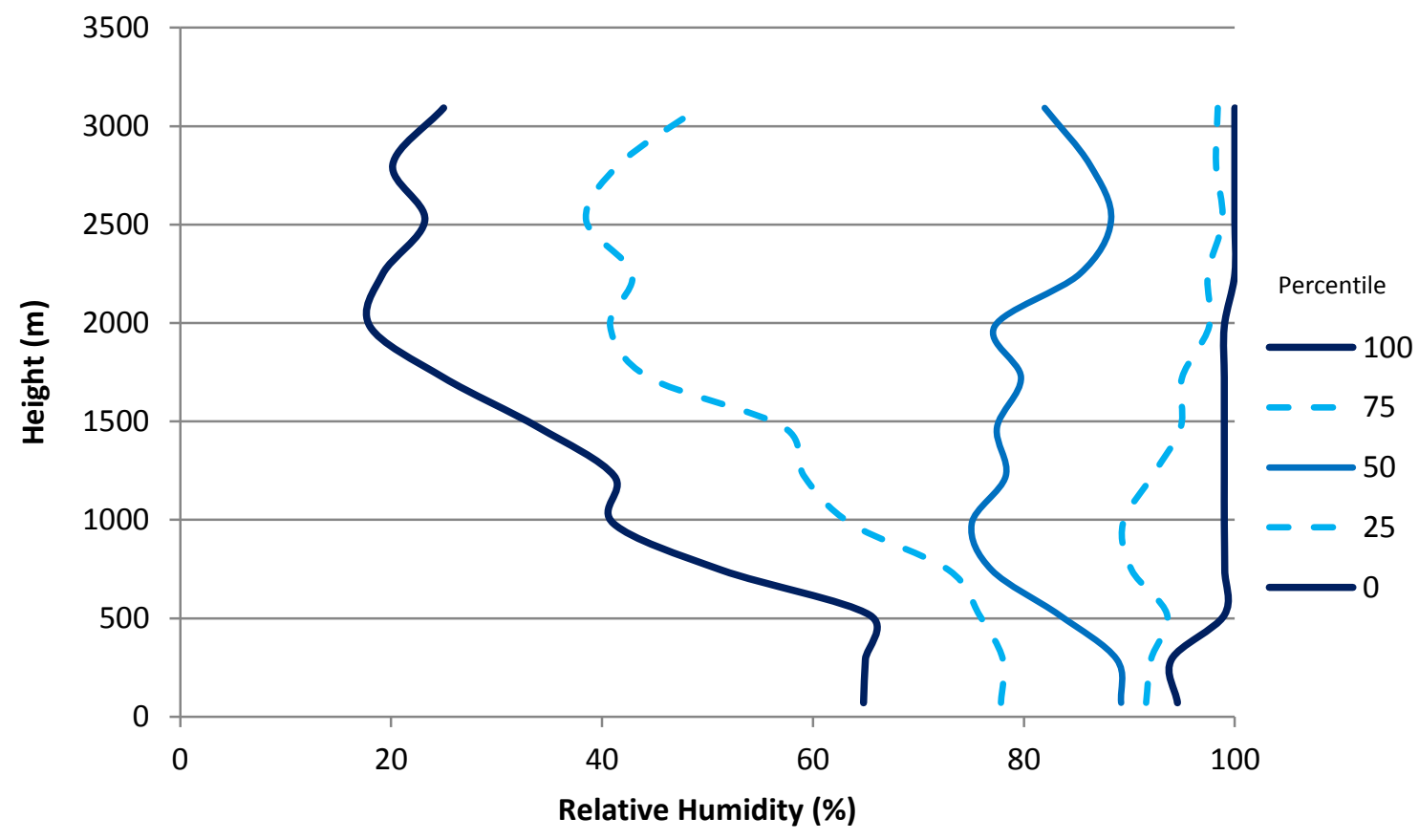

Figure 3.12.2. Relative Humidity Profile May 30, 2013. 


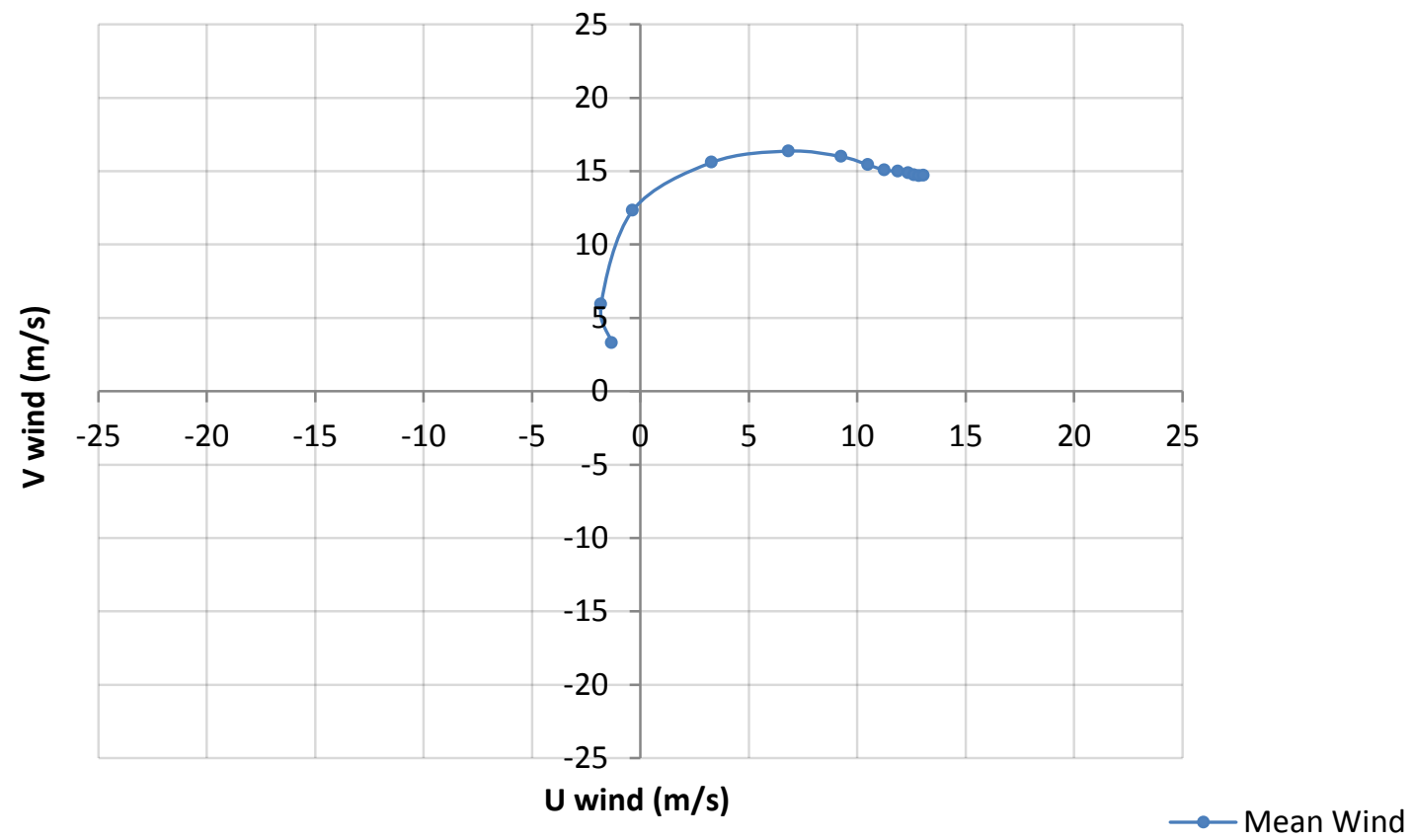

Figure 3.12.3. Hodograph May 30, 2013. 


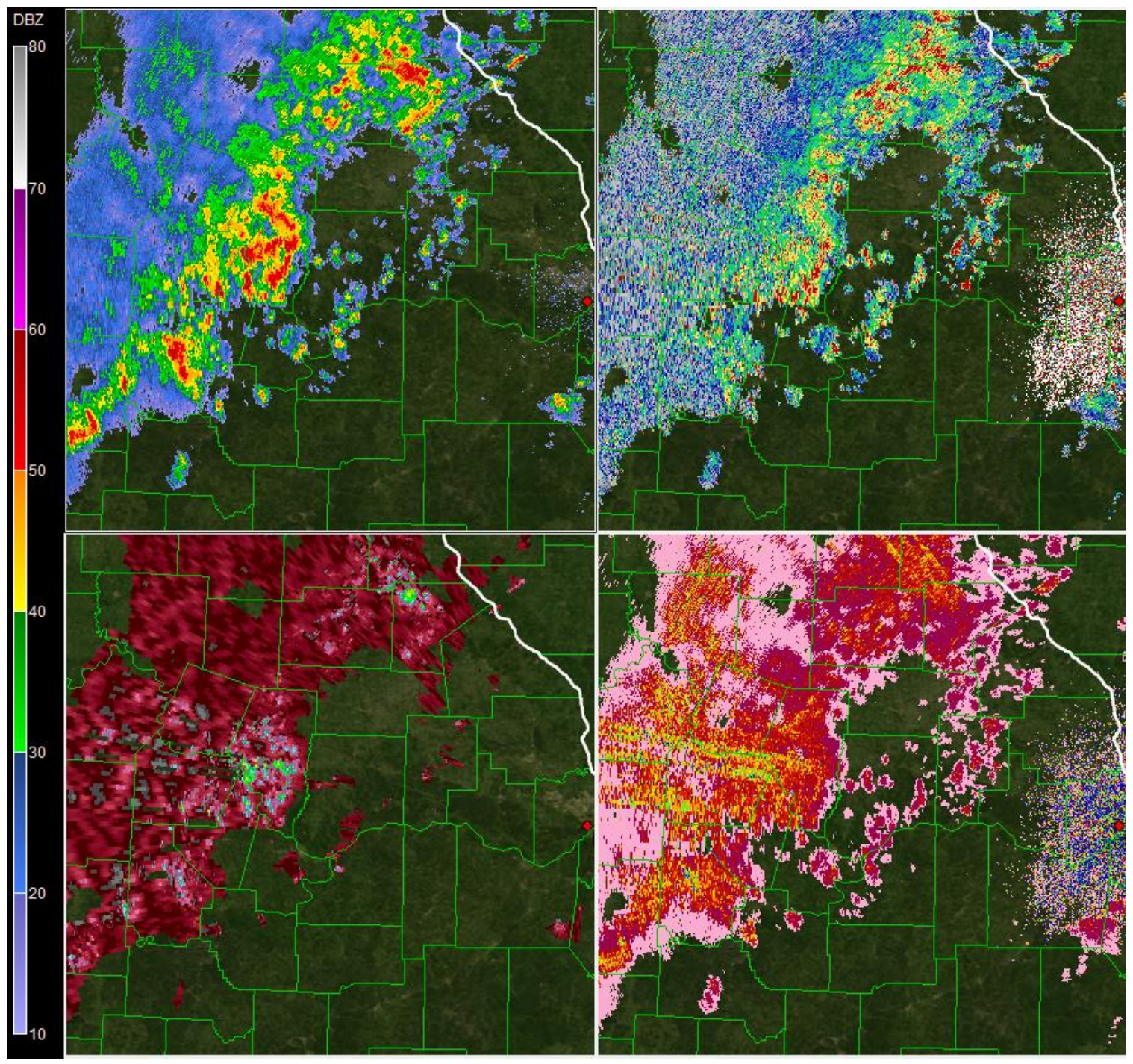

Figure 3.12.4. Four Panel Radar Image for 2044 UTC May 30, 2013. The top left is reflectivity $(Z)$, top right is differential reflectivity $\left(Z_{D R}\right)$, bottom left is specific phase differential $\left(K_{D P}\right)$, and bottom right is correlation coefficient. The scale on the left corresponds to the top left image (Z). The elevation angle used here was $0.5^{\circ}$. 
Table 3.12.1 Conventional Techniques, May 30, 2013

\begin{tabular}{|c|c|c|c|c|c|}
\hline$Z=a R^{b}$ & $\#$ & $\mathrm{FB}(\%)$ & $\mathrm{FAD}(\%)$ & FRMSE(\%) & FSD(\%) \\
\hline 1 & 114 & -9.85 & 43.19 & 58.95 & 58.12 \\
\hline 2 & 113 & 4.87 & 48.25 & 65.42 & 65.24 \\
\hline 3 & 113 & 114.87 & 128.27 & 200.87 & 164.78 \\
\hline 4 & 113 & -4.96 & 45.24 & 61.36 & 61.15 \\
\hline 5 & 112 & -31.32 & 47.92 & 67.61 & 59.92 \\
\hline \multicolumn{6}{|c|}{$R\left(K_{D P}\right)=a\left|K_{D P}\right|^{b} \operatorname{sign}\left(K_{D P}\right)$} \\
\hline 6 & 120 & 16.75 & 76.09 & 117.29 & 116.09 \\
\hline 7 & 121 & 26.09 & 81.72 & 126.63 & 123.91 \\
\hline 8 & 121 & 22.54 & 77.60 & 117.65 & 115.47 \\
\hline 9 & 120 & 1.86 & 66.84 & 100.72 & 100.71 \\
\hline 10 & 121 & 16.66 & 75.34 & 115.79 & 114.59 \\
\hline 11 & 121 & 10.18 & 71.02 & 107.82 & 107.34 \\
\hline \multicolumn{6}{|c|}{$R\left(Z, Z_{D R}\right)=a Z^{b} Z_{d r}^{c}$} \\
\hline 12 & 113 & -30.23 & 60.87 & 81.79 & 76.00 \\
\hline 13 & 113 & -38.64 & 70.26 & 94.84 & 86.61 \\
\hline 14 & 113 & -37.66 & 56.93 & 76.76 & 66.89 \\
\hline 15 & 113 & -34.20 & 52.92 & 71.59 & 62.90 \\
\hline 16 & 113 & -38.24 & 56.62 & 76.51 & 66.26 \\
\hline \multicolumn{6}{|c|}{$R\left(K_{D P}, Z_{D R}\right)=a\left|K_{D P}\right|^{b} Z_{d r}^{c} \operatorname{sign}\left(K_{D P}\right)$} \\
\hline 17 & 121 & 30.51 & 90.93 & 141.31 & 137.98 \\
\hline 18 & 121 & 42.18 & 105.79 & 169.22 & 163.88 \\
\hline 19 & 119 & 8.80 & 70.08 & 105.52 & 105.15 \\
\hline 20 & 120 & 24.00 & 80.68 & 122.98 & 120.61 \\
\hline \multicolumn{6}{|l|}{ Synthetic } \\
\hline 21 & 111 & -5.98 & 59.59 & 84.68 & 84.47 \\
\hline
\end{tabular}


Table 3.12.2 Trace Techniques, May 30, 2013

\begin{tabular}{|c|c|c|c|c|c|c|}
\hline & $Z_{D R}+$ & $\#$ & $\mathrm{FB}(\%)$ & $\mathrm{FAD}(\%)$ & FRMSE(\%) & FSD(\%) \\
\hline \multirow[t]{14}{*}{ T1 } & 0 & 107 & -7.49 & 50.44 & 75.22 & 74.85 \\
\hline & 0.2 & 107 & -7.36 & 49.29 & 75.05 & 74.69 \\
\hline & 0.3 & 107 & -7.29 & 48.99 & 75.36 & 75.01 \\
\hline & 0.4 & 106 & -7.14 & 48.98 & 75.81 & 75.48 \\
\hline & 0.5 & 106 & -7.05 & 49.13 & 76.83 & 76.51 \\
\hline & 0.6 & 106 & -7.06 & 49.30 & 77.97 & 77.65 \\
\hline & 0.7 & 106 & -7.25 & 49.43 & 79.14 & 78.80 \\
\hline & 0.8 & 106 & -7.55 & 49.52 & 80.37 & 80.01 \\
\hline & 0.9 & 106 & -8.01 & 49.69 & 81.78 & 81.39 \\
\hline & 1 & 106 & -8.63 & 49.88 & 83.40 & 82.96 \\
\hline & 1.1 & 106 & -9.64 & 49.93 & 84.55 & 84.00 \\
\hline & 1.5 & 105 & -16.91 & 48.28 & 82.94 & 81.20 \\
\hline & 2 & 103 & -29.71 & 47.86 & 76.22 & 70.19 \\
\hline & 2.6 & 102 & -43.41 & 52.80 & 76.72 & 63.25 \\
\hline \multirow[t]{12}{*}{$\mathrm{T} 2$} & 0.4 & 108 & 77.04 & 88.17 & 133.62 & 109.18 \\
\hline & 0.6 & 106 & 66.41 & 81.10 & 123.11 & 103.66 \\
\hline & 0.8 & 106 & 57.18 & 75.81 & 116.22 & 101.18 \\
\hline & 1 & 105 & 49.18 & 71.84 & 110.40 & 98.84 \\
\hline & 1.2 & 104 & 41.08 & 67.84 & 103.55 & 95.05 \\
\hline & 1.4 & 104 & 32.30 & 63.77 & 96.76 & 91.21 \\
\hline & 1.6 & 104 & 23.10 & 59.91 & 89.22 & 86.18 \\
\hline & 1.8 & 104 & 13.32 & 56.10 & 81.02 & 79.92 \\
\hline & 2 & 103 & 3.91 & 52.89 & 73.84 & 73.74 \\
\hline & 2.2 & 103 & -4.74 & 50.04 & 69.09 & 68.93 \\
\hline & 2.4 & 103 & -12.62 & 47.92 & 66.40 & 65.19 \\
\hline & 2.6 & 103 & -19.73 & 46.93 & 65.55 & 62.51 \\
\hline \multirow[t]{14}{*}{ T3 } & 0 & 110 & -8.52 & 49.44 & 75.25 & 74.76 \\
\hline & 0.2 & 109 & -8.73 & 47.54 & 73.17 & 72.64 \\
\hline & 0.3 & 109 & -8.65 & 47.25 & 73.35 & 72.84 \\
\hline & 0.4 & 107 & -8.56 & 47.34 & 73.46 & 72.96 \\
\hline & 0.5 & 107 & -8.49 & 47.63 & 74.57 & 74.09 \\
\hline & 0.6 & 106 & -8.37 & 48.26 & 75.87 & 75.41 \\
\hline & 0.7 & 105 & -8.39 & 48.87 & 77.21 & 76.75 \\
\hline & 0.8 & 105 & -8.87 & 49.12 & 77.96 & 77.46 \\
\hline & 0.9 & 105 & -9.44 & 49.30 & 78.65 & 78.08 \\
\hline & 1 & 105 & -10.30 & 49.19 & 78.64 & 77.96 \\
\hline & 1.1 & 105 & -11.42 & 48.87 & 78.11 & 77.27 \\
\hline & 1.5 & 104 & -18.63 & 47.50 & 75.03 & 72.69 \\
\hline & 2 & 103 & -31.17 & 47.60 & 71.57 & 64.43 \\
\hline & 2.6 & 103 & -44.78 & 51.66 & 74.78 & 59.89 \\
\hline
\end{tabular}


Table 3.12.2 Trace Techniques, May 30, 2013 (Continued)

\begin{tabular}{ccccccc}
\hline & $Z_{D R}+$ & $\#$ & $\mathrm{FB}(\%)$ & $\mathrm{FAD}(\%)$ & $\mathrm{FRMSE}(\%)$ & $\mathrm{FSD}(\%)$ \\
\hline $\mathrm{T} 4$ & 0.4 & 111 & 76.94 & 85.98 & 128.37 & 102.75 \\
& 0.6 & 109 & 66.33 & 78.88 & 117.31 & 96.76 \\
0.8 & 108 & 57.09 & 73.46 & 108.96 & 92.81 \\
& 1 & 107 & 49.10 & 68.74 & 102.29 & 89.73 \\
& 1.2 & 105 & 41.01 & 63.97 & 94.69 & 85.35 \\
1.4 & 105 & 32.24 & 59.08 & 87.84 & 81.71 \\
1.6 & 104 & 23.04 & 54.56 & 80.10 & 76.71 \\
& 1.8 & 104 & 13.27 & 50.40 & 72.66 & 71.44 \\
2 & 103 & 3.86 & 47.04 & 66.47 & 66.36 \\
& 103 & -4.78 & 44.43 & 62.72 & 62.53 \\
& 1.2 & -12.65 & 43.26 & 61.06 & 59.73 \\
& 2.4 & 103 & -19.76 & 43.06 & 61.19 & 57.91 \\
\hline 2.6 & 103 & 74.01 & 81.96 & 117.09 & 90.73 \\
& 0.4 & 107 & 64.91 & 76.02 & 109.54 & 88.24 \\
& 107 & 57.30 & 72.15 & 104.54 & 87.44 \\
0.6 & 106 & 50.54 & 68.64 & 102.29 & 88.94 \\
1 & 106 & 43.04 & 64.18 & 98.99 & 89.15 \\
& 106 & 34.37 & 58.62 & 93.35 & 86.79 \\
1.2 & 106 & 24.92 & 52.15 & 85.40 & 81.68 \\
1.4 & 106 & 15.23 & 47.19 & 77.48 & 75.97 \\
1.6 & 105 & 5.87 & 43.99 & 71.27 & 71.03 \\
1.8 & 105 & -2.85 & 42.66 & 66.31 & 66.25 \\
2 & 105 & -10.73 & 42.23 & 63.77 & 62.86 \\
2.2 & 103 & -17.87 & 43.01 & 63.28 & 60.70 \\
2.4 & 102 & & & & \\
2.6 & 102 & & & &
\end{tabular}




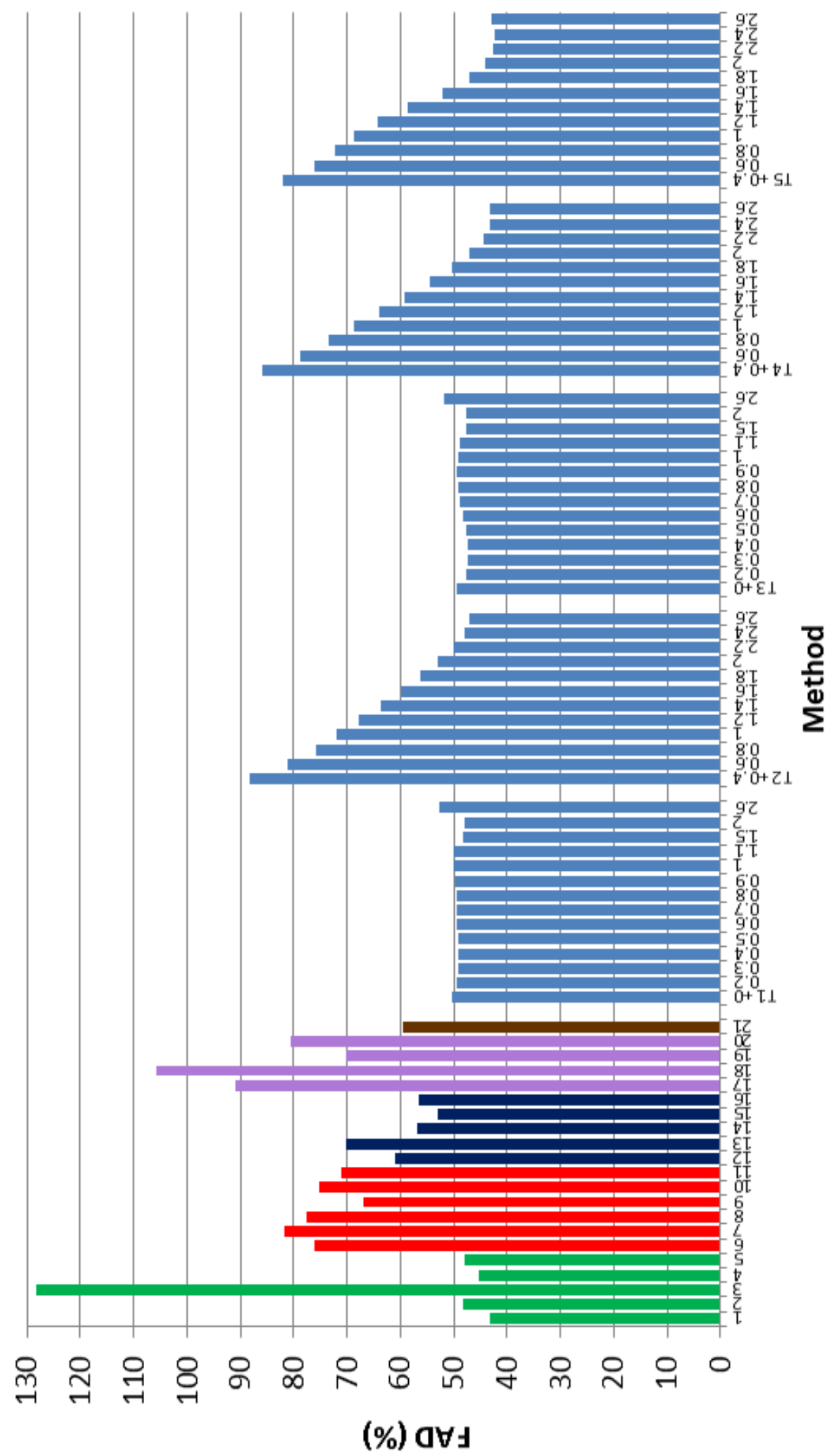

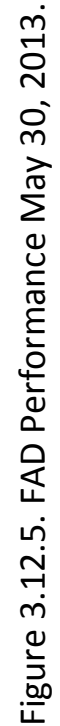




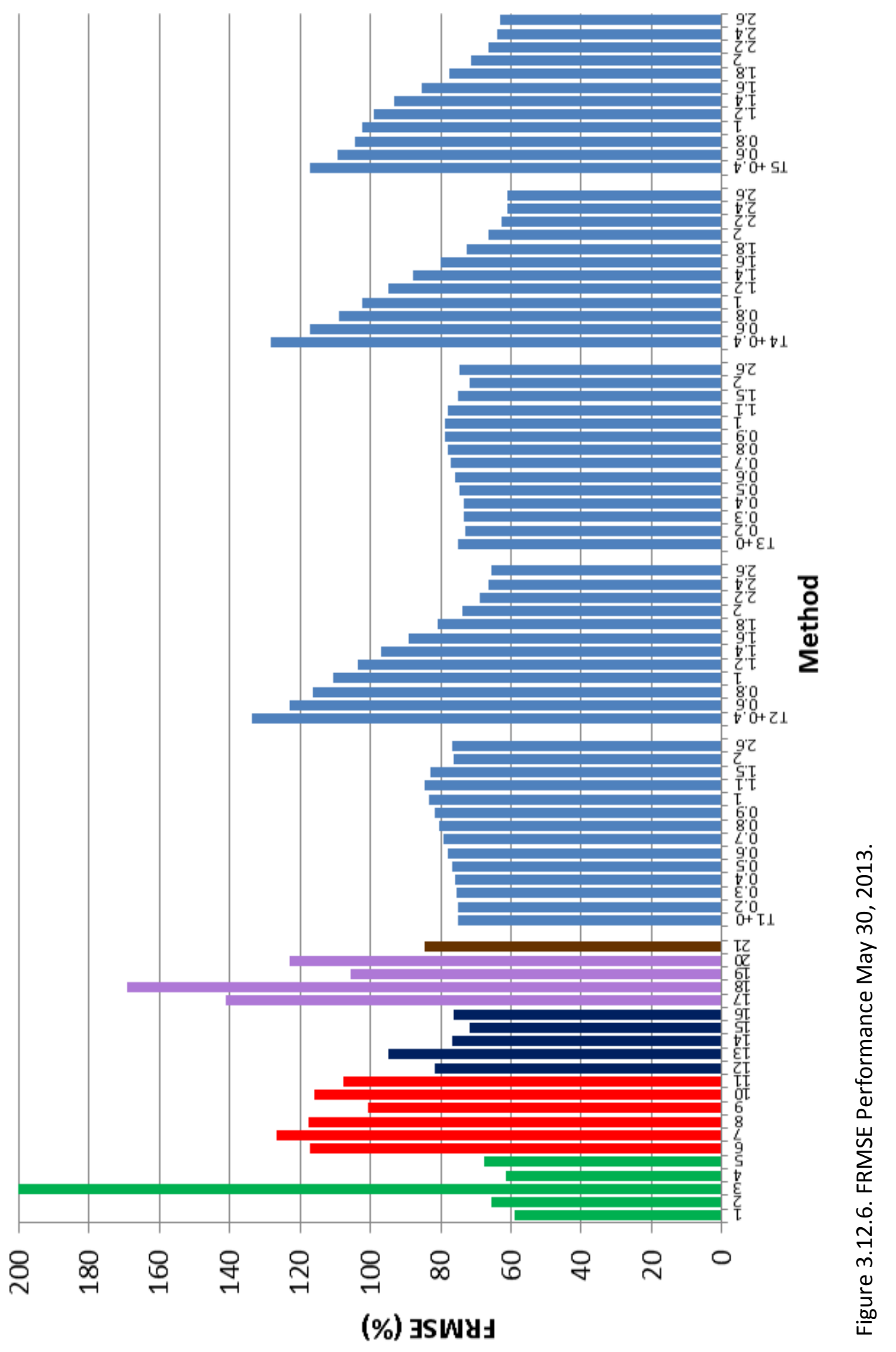




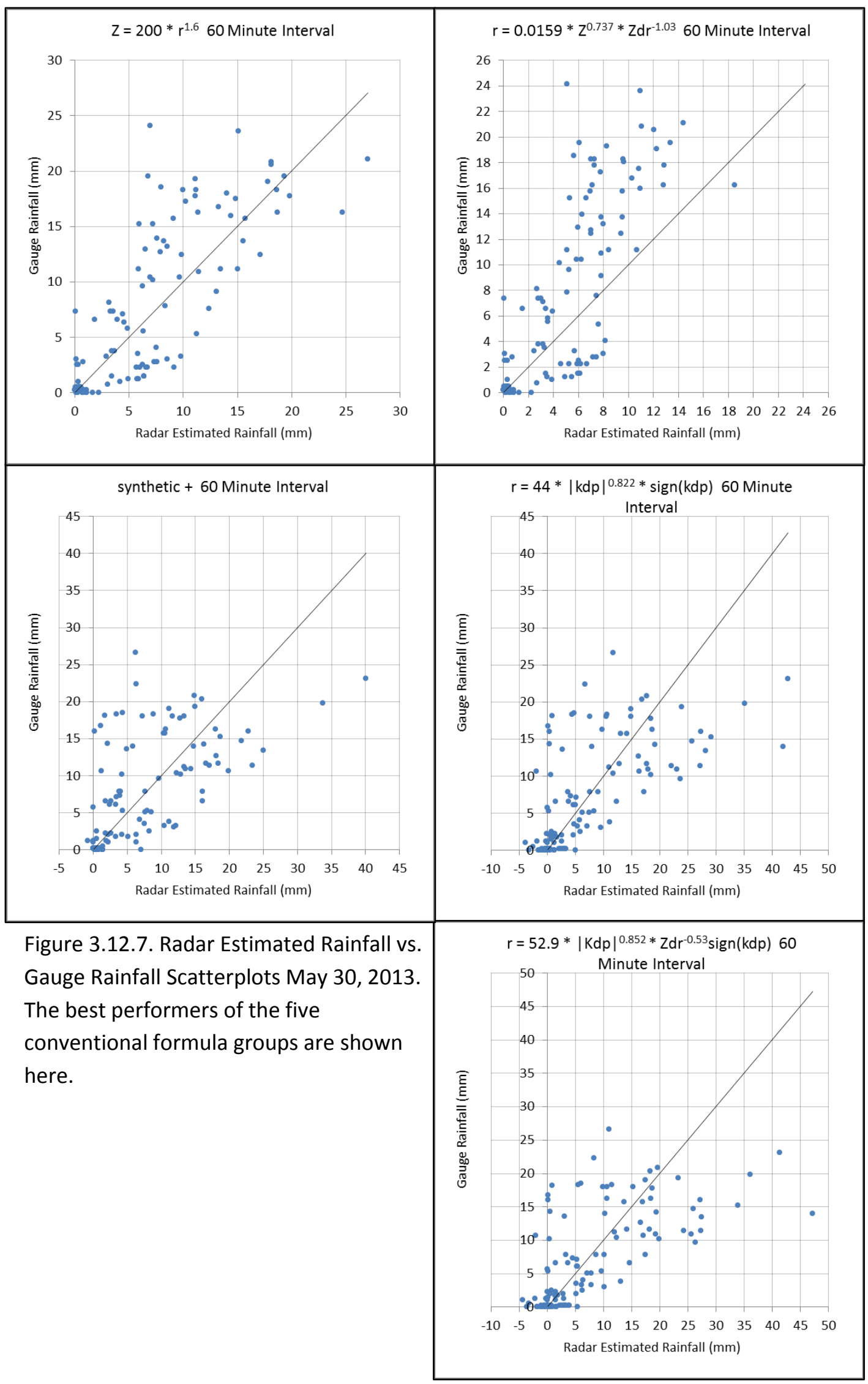



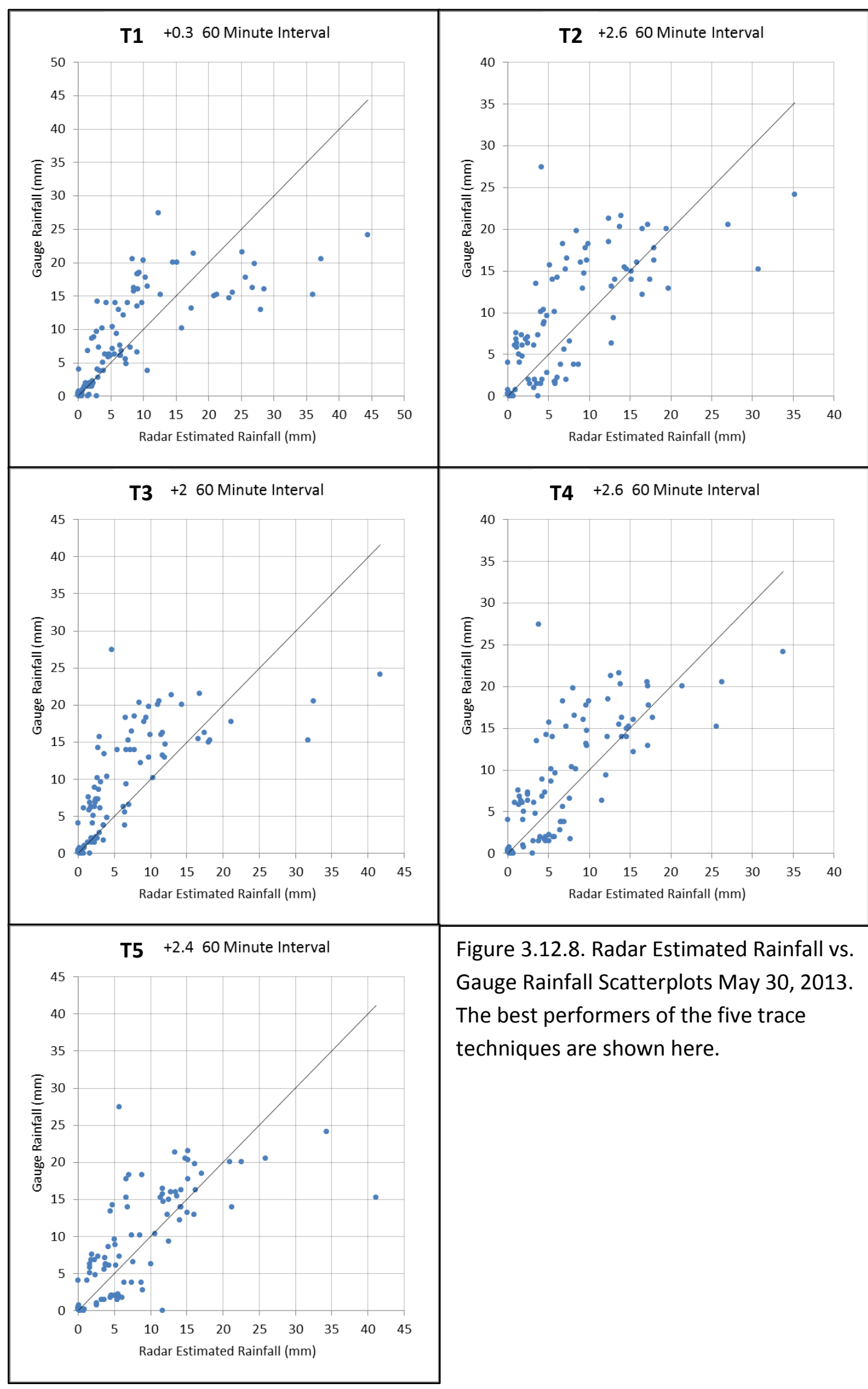

Figure 3.12.8. Radar Estimated Rainfall vs. Gauge Rainfall Scatterplots May 30, 2013. The best performers of the five trace techniques are shown here. 


\subsection{Statistical Analysis and Discussion}

To determine the overall performance of each individual method for rainfall estimation, a statistical analysis was done. Each of the rainfall events was given equal weight for this analysis. The parameters chosen for analysis were bias, FAD, and FRMSE. Along with the mean values, five percentiles were calculated: $0^{\text {th }}, 25^{\text {th }}, 50^{\text {th }}, 75^{\text {th }}$, and $100^{\text {th }}$. The $0^{\text {th }}$ percentile for a method was equivalent to the minimum value of the parameter in question over all events examined. The $50^{\text {th }}$ percentile was the median value and the $100^{\text {th }}$ percentile was the maximum. For example, if method " $A$ " showed a FAD of $30 \%$ for a first event, $40 \%$ for a second event, and $50 \%$ for a third event and there were three total events, the $0^{\text {th }}$ percentile for the FAD parameter would be $30 \%$, the $50^{\text {th }}$ percentile would be $40 \%$, and the $100^{\text {th }}$ percentile would be $50 \%$.

The choice to weight each event equally was made for several reasons. Successful rainfall estimation under different environmental conditions was the primary reason for weighting each event equally. This approach rewards a method for performing well in two shorter rainfall events, rather than one prolonged event. The other issues at play were time and technical constraints. Each of the April, 2012, events had an environmental factor that set the events apart from one another. The atmosphere on April 13, 2012, had a very dry layer, while the two subsequent days did not. On April 15, 2012, the precipitation occurred ahead of a cold front moving through the area, while the precipitation for the other two days was not associated with this front. The other candidate for "splitting" was the May 30-31, 2013, event. The wide range of relative humidity values was indicative of a very dynamic atmosphere. The storms during this 
event, however, were not separated by significant time periods, as they were for the April, 2012, events.

Table 3.13.1 shows the mean and percentile performance analysis for the conventional formulas with respect to the FAD parameter. By median values, (1), (2), and (4) performed nearly identically. Mean values were only slightly different. Table 3.14.1 shows the mean and percentile performance analysis for the conventional formulas with respect to the FRMSE parameter. These values were generally higher, due to the outlier penalty introduced by the squaring. The same general patterns found in Table 3.13.1 were also present in Table 3.14.1.

Table 3.13.2 shows the mean and percentile performance analysis for the trace techniques with respect to the FAD parameter, while Table 3.14.2 shows the same analysis using the FRMSE parameter. Figure 3.13.1 shows the mean FAD performance of each of the five trace techniques as a function of $Z_{D R}$ adjustment. Figure 3.13 .2 is identical to Figure 3.13.1 except it shows the median FAD performance. Figure 3.13.3 and Figure 3.13.4 are the FRMSE equivalents to Figure 3.13.1 and 3.13.2. Figure 3.13.5 and Figure 3.13.6 are the Bias equivalents. For the FRMSE and FAD charts, the dotted line represents the best performing conventional formula. The optimal T1 and T3 techniques show some improvement over the best performing conventional formulas on the FRMSE and FAD charts.

At first glance all three pairs of graphs show a clear divide between the two techniques that account for evaporation and the other trace techniques. As a whole, T1 and T3 
performed best at roughly the $+1 Z_{D R}$ adjustment, while the other techniques performed best near the $+2 Z_{D R}$ adjustment. The optimal evaporation techniques significantly outperformed the optimal no-evaporation techniques, and even the conventional formulas. The difference in performance with respect to the mean FAD and FRMSE was much greater than the difference with respect to the median FAD and FRMSE; this was likely due to the hail events significantly skewing the means for the conventional formulas.

The $+2 Z_{D R}$ adjustment required for optimal performance for the no-evaporation techniques was not very realistic. It essentially was a form of evaporation adjustment in itself, in a one size fits all format. Adjusting the $Z_{D R}$ as a means to account for evaporation also reduced the effectiveness of the hail adjustment function.

The conclusion can be drawn from these results that accounting for evaporation is necessary to accurately estimate rainfall amounts with radar. Significant improvements to overall FAD and FRMSE were made when evaporation was taken into account. High resolution model data describing atmospheric humidity can serve as a useful tool for radar-derived rainfall estimation.

Overall any improvement due to accounting for drift was insignificant. However, accounting for drift did result in noticeable performance improvement on September 7, 2012 and April 17, 2013. A very specific set of environmental conditions is necessary for drift to have significant theoretical effect. If the storm is moving in the same direction as the wind and stays at constant strength, the drift calculations only alter the time at 
which the drops reach the ground. As discussed in Chapter 1, significant directional shear would be necessary to alter the paths of the drops away from the path of the storm. The degree to which the storms are scattered in nature also has theoretical implications on precipitation drift; if the precipitation covers a very broad area, it is unlikely that precipitation will drift to locations that are not similarly covered in precipitation.

Another factor for examination is time lag of precipitation. All of the conventional formulas and the $\mathrm{T} 2$ trace technique assumed precipitation instantly reaches the ground. Theoretically, the time it takes for the precipitation to reach the ground may alter which time slot the precipitation is recorded in, and thus affect the results. On an hourly scale, there are likely minimal amounts of this kind of clipping, but finer time scales may be more affected. The T2 and T4 techniques both did not take into account evaporation or precipitation drift, but the precipitation in the T4 technique did take time to reach the ground. The T4 technique did perform slightly better, but the difference was not significant. 
Table 3.13.1 Conventional Formulas, FAD (\%) by Percentile

\begin{tabular}{|c|c|c|c|c|c|c|}
\hline $\begin{array}{l}Z \\
=a R^{b}\end{array}$ & Mean & $0^{\text {th }}$ & $25^{\text {th }}$ & $50^{\text {th }}$ & $75^{\text {th }}$ & $100^{\text {th }}$ \\
\hline 1 & 46.41 & 31.72 & 40.90 & 45.46 & 49.37 & 64.49 \\
\hline 2 & 48.25 & 35.65 & 36.48 & 45.97 & 58.95 & 76.21 \\
\hline 3 & 111.72 & 39.30 & 63.61 & 109.78 & 158.08 & 192.10 \\
\hline 4 & 46.41 & 31.96 & 37.93 & 45.43 & 54.13 & 70.13 \\
\hline 5 & 49.71 & 24.57 & 46.18 & 50.03 & 55.60 & 68.78 \\
\hline \multicolumn{7}{|c|}{$R\left(K_{D P}\right)=a\left|K_{D P}\right|^{b} \operatorname{sign}\left(K_{D P}\right)$} \\
\hline 6 & 115.39 & 45.12 & 67.11 & 101.48 & 176.02 & 212.88 \\
\hline 7 & 127.62 & 54.13 & 67.84 & 113.86 & 196.39 & 235.74 \\
\hline 8 & 128.39 & 52.63 & 67.30 & 113.13 & 200.57 & 240.24 \\
\hline 9 & 101.50 & 37.43 & 65.43 & 85.34 & 154.33 & 188.04 \\
\hline 10 & 116.84 & 45.64 & 66.45 & 102.55 & 179.52 & 216.95 \\
\hline 11 & 110.87 & 41.69 & 65.33 & 95.74 & 170.57 & 206.75 \\
\hline \multicolumn{7}{|c|}{$R\left(Z, Z_{D R}\right)=a Z^{b} Z_{d r}^{c}$} \\
\hline 12 & 53.03 & 33.85 & 36.55 & 53.33 & 64.38 & 75.15 \\
\hline 13 & 59.31 & 35.68 & 39.75 & 59.21 & 70.89 & 87.34 \\
\hline 14 & 48.26 & 33.98 & 38.12 & 48.98 & 55.97 & 67.28 \\
\hline 15 & 47.13 & 31.53 & 37.92 & 49.19 & 53.83 & 65.37 \\
\hline 16 & 48.22 & 34.12 & 37.43 & 49.40 & 55.88 & 67.41 \\
\hline \multicolumn{7}{|c|}{$R\left(K_{D P}, Z_{D R}\right)=a\left|K_{D P}\right|^{b} Z_{d r}^{c} \operatorname{sign}\left(K_{D P}\right)$} \\
\hline 17 & 161.97 & 53.39 & 77.26 & 146.66 & 252.16 & 326.26 \\
\hline 18 & 211.48 & 63.87 & 91.70 & 190.88 & 328.64 & 452.05 \\
\hline 19 & 114.66 & 39.97 & 66.92 & 98.91 & 178.52 & 217.09 \\
\hline 20 & 135.38 & 48.47 & 69.94 & 120.89 & 212.39 & 258.59 \\
\hline \multicolumn{7}{|c|}{ Synthetic } \\
\hline 21 & 56.04 & 36.42 & 42.44 & 59.61 & 60.63 & 79.41 \\
\hline
\end{tabular}


Table 3.13.2 Trace Techniques, FAD (\%) by Percentile

\begin{tabular}{|c|c|c|c|c|c|c|c|}
\hline & $Z_{D R}+$ & Mean & $0^{\text {th }}$ & $25^{\text {th }}$ & $50^{\text {th }}$ & $75^{\text {th }}$ & $100^{\text {th }}$ \\
\hline \multirow[t]{14}{*}{ T1 } & 0 & 77.51 & 20.41 & 45.50 & 63.42 & 96.73 & 187.46 \\
\hline & 0.2 & 66.31 & 19.48 & 41.63 & 55.06 & 71.42 & 151.87 \\
\hline & 0.3 & 61.38 & 19.28 & 39.75 & 51.43 & 61.71 & 134.75 \\
\hline & 0.4 & 56.95 & 18.99 & 38.73 & 49.55 & 57.05 & 121.69 \\
\hline & 0.5 & 53.13 & 18.89 & 37.99 & 45.72 & 54.00 & 112.42 \\
\hline & 0.6 & 49.81 & 19.64 & 36.60 & 42.43 & 52.05 & 103.98 \\
\hline & 0.7 & 46.84 & 20.91 & 36.06 & 39.61 & 50.32 & 95.62 \\
\hline & 0.8 & 44.43 & 22.52 & 34.68 & 38.76 & 49.22 & 88.22 \\
\hline & 0.9 & 42.60 & 23.99 & 33.22 & 38.94 & 48.91 & 81.97 \\
\hline & 1 & 41.35 & 25.76 & 32.01 & 38.23 & 48.66 & 75.86 \\
\hline & 1.1 & 40.76 & 26.75 & 32.24 & 39.10 & 46.04 & 70.58 \\
\hline & 1.5 & 42.22 & 29.92 & 33.30 & 42.46 & 48.53 & 58.16 \\
\hline & 2 & 47.36 & 35.51 & 37.00 & 44.64 & 56.93 & 73.91 \\
\hline & 2.6 & 55.29 & 42.76 & 45.96 & 51.69 & 66.43 & 78.03 \\
\hline \multirow[t]{12}{*}{ T2 } & 0.4 & 111.25 & 53.68 & 81.07 & 94.22 & 147.29 & 210.00 \\
\hline & 0.6 & 91.06 & 39.86 & 65.16 & 81.04 & 116.14 & 162.52 \\
\hline & 0.8 & 76.36 & 35.06 & 61.62 & 70.81 & 92.87 & 125.62 \\
\hline & 1 & 65.71 & 36.82 & 50.61 & 66.21 & 76.23 & 98.64 \\
\hline & 1.2 & 58.20 & 36.32 & 43.95 & 57.56 & 71.61 & 82.13 \\
\hline & 1.4 & 53.43 & 31.27 & 45.33 & 52.60 & 62.20 & 78.07 \\
\hline & 1.6 & 50.48 & 26.32 & 41.11 & 50.15 & 56.90 & 84.76 \\
\hline & 1.8 & 49.40 & 24.44 & 37.55 & 49.91 & 55.67 & 91.90 \\
\hline & 2 & 49.90 & 27.56 & 36.57 & 49.03 & 57.99 & 97.38 \\
\hline & 2.2 & 51.07 & 25.57 & 38.00 & 49.79 & 61.08 & 97.26 \\
\hline & 2.4 & 52.86 & 25.77 & 39.39 & 49.14 & 64.36 & 97.87 \\
\hline & 2.6 & 55.26 & 28.05 & 42.08 & 51.09 & 67.38 & 99.35 \\
\hline \multirow[t]{14}{*}{ T3 } & 0 & 74.50 & 21.69 & 36.22 & 66.26 & 92.48 & 184.40 \\
\hline & 0.2 & 63.90 & 22.15 & 35.57 & 58.82 & 73.09 & 147.87 \\
\hline & 0.3 & 59.17 & 22.44 & 35.09 & 53.62 & 67.83 & 130.63 \\
\hline & 0.4 & 55.08 & 23.09 & 34.48 & 49.22 & 63.89 & 114.70 \\
\hline & 0.5 & 51.51 & 24.08 & 33.87 & 48.42 & 60.54 & 100.49 \\
\hline & 0.6 & 48.46 & 25.07 & 31.55 & 46.31 & 57.31 & 90.62 \\
\hline & 0.7 & 45.97 & 26.25 & 30.35 & 43.64 & 54.32 & 82.59 \\
\hline & 0.8 & 44.02 & 26.94 & 30.43 & 41.65 & 52.12 & 75.41 \\
\hline & 0.9 & 42.65 & 27.53 & 31.69 & 41.32 & 50.18 & 69.09 \\
\hline & 1 & 41.85 & 28.29 & 32.80 & 42.07 & 48.97 & 63.54 \\
\hline & 1.1 & 41.38 & 28.38 & 31.80 & 43.12 & 48.27 & 58.33 \\
\hline & 1.5 & 42.28 & 27.58 & 35.87 & 42.66 & 48.18 & 52.69 \\
\hline & 2 & 48.18 & 35.36 & 39.12 & 47.60 & 58.71 & 67.23 \\
\hline & 2.6 & 56.78 & 45.00 & 48.21 & 54.30 & 68.18 & 70.53 \\
\hline
\end{tabular}


Table 3.13.2 Trace Techniques, FAD (\%) by Percentile (Continued)

\begin{tabular}{|c|c|c|c|c|c|c|c|}
\hline & $Z_{D R}+$ & Mean & $0^{\text {th }}$ & $25^{\text {th }}$ & $50^{\text {th }}$ & $75^{\text {th }}$ & $100^{\text {th }}$ \\
\hline \multirow[t]{12}{*}{ T4 } & 0.4 & 106.45 & 50.28 & 74.87 & 85.03 & 145.46 & 206.41 \\
\hline & 0.6 & 86.52 & 34.49 & 60.44 & 76.35 & 113.11 & 161.45 \\
\hline & 0.8 & 72.24 & 31.83 & 49.30 & 67.51 & 87.02 & 124.93 \\
\hline & 1 & 62.27 & 34.48 & 42.36 & 62.71 & 70.14 & 98.09 \\
\hline & 1.2 & 55.39 & 31.50 & 42.04 & 53.99 & 67.80 & 83.31 \\
\hline & 1.4 & 50.49 & 28.45 & 44.21 & 48.27 & 58.26 & 72.85 \\
\hline & 1.6 & 47.55 & 26.15 & 39.22 & 44.49 & 54.34 & 78.43 \\
\hline & 1.8 & 46.91 & 28.95 & 35.05 & 45.42 & 53.58 & 85.29 \\
\hline & 2 & 47.92 & 30.05 & 34.40 & 46.75 & 56.59 & 90.26 \\
\hline & 2.2 & 49.16 & 27.82 & 37.11 & 43.20 & 60.28 & 89.90 \\
\hline & 2.4 & 50.82 & 28.59 & 38.97 & 43.24 & 63.69 & 88.93 \\
\hline & 2.6 & 53.30 & 31.76 & 40.38 & 46.40 & 66.79 & 88.71 \\
\hline \multirow[t]{12}{*}{ T5 } & 0.4 & 111.48 & 40.77 & 69.37 & 98.35 & 155.54 & 188.04 \\
\hline & 0.6 & 91.62 & 36.71 & 62.91 & 81.12 & 121.51 & 153.76 \\
\hline & 0.8 & 76.30 & 34.05 & 54.43 & 73.05 & 99.89 & 126.82 \\
\hline & 1 & 64.79 & 32.22 & 46.17 & 66.55 & 81.10 & 105.51 \\
\hline & 1.2 & 57.06 & 30.32 & 42.59 & 56.91 & 69.36 & 85.76 \\
\hline & 1.4 & 52.50 & 28.10 & 43.22 & 52.84 & 59.86 & 78.05 \\
\hline & 1.6 & 49.26 & 24.68 & 39.94 & 47.96 & 56.84 & 85.39 \\
\hline & 1.8 & 47.73 & 22.05 & 38.21 & 46.91 & 52.69 & 93.44 \\
\hline & 2 & 47.43 & 26.39 & 32.03 & 44.42 & 55.10 & 98.83 \\
\hline & 2.2 & 48.05 & 26.85 & 33.07 & 42.41 & 58.78 & 99.80 \\
\hline & 2.4 & 49.51 & 29.11 & 35.59 & 41.47 & 61.86 & 100.78 \\
\hline & 2.6 & 51.60 & 32.18 & 37.17 & 42.10 & 64.30 & 101.06 \\
\hline
\end{tabular}


Table 3.14.1 Conventional Formulas, FRMSE (\%) by Percentile

\begin{tabular}{|c|c|c|c|c|c|c|}
\hline $\begin{array}{l}Z \\
=a R^{b}\end{array}$ & Mean & $0^{\text {th }}$ & $25^{\text {th }}$ & $50^{\text {th }}$ & $75^{\text {th }}$ & $100^{\text {th }}$ \\
\hline 1 & 67.35 & 47.56 & 51.91 & 64.06 & 78.01 & 113.48 \\
\hline 2 & 72.76 & 46.98 & 57.82 & 64.53 & 78.24 & 142.59 \\
\hline 3 & 179.28 & 52.89 & 84.85 & 178.46 & 218.85 & 363.50 \\
\hline 4 & 68.95 & 40.91 & 54.81 & 63.73 & 73.39 & 128.58 \\
\hline 5 & 73.24 & 35.82 & 61.07 & 70.97 & 87.15 & 113.94 \\
\hline \multicolumn{7}{|c|}{$R\left(K_{D P}\right)=a\left|K_{D P}\right|^{b} \operatorname{sign}\left(K_{D P}\right)$} \\
\hline 6 & 157.08 & 66.54 & 91.53 & 147.38 & 228.74 & 278.71 \\
\hline 7 & 173.60 & 77.57 & 92.53 & 164.77 & 255.41 & 308.69 \\
\hline 8 & 172.61 & 74.20 & 89.69 & 161.56 & 259.98 & 311.52 \\
\hline 9 & 137.18 & 57.47 & 88.47 & 123.82 & 200.02 & 241.39 \\
\hline 10 & 158.37 & 66.78 & 90.31 & 147.65 & 232.98 & 283.07 \\
\hline 11 & 149.68 & 61.62 & 88.40 & 137.28 & 221.01 & 268.57 \\
\hline \multicolumn{7}{|c|}{$R\left(Z, Z_{D R}\right)=a Z^{b} Z_{d r}^{c}$} \\
\hline 12 & 77.83 & 49.22 & 56.16 & 80.62 & 93.53 & 109.07 \\
\hline 13 & 89.05 & 48.61 & 62.90 & 93.09 & 109.21 & 132.68 \\
\hline 14 & 70.77 & 48.01 & 52.90 & 71.92 & 79.72 & 109.83 \\
\hline 15 & 69.15 & 41.55 & 54.97 & 71.18 & 78.93 & 104.09 \\
\hline 16 & 70.86 & 44.31 & 54.21 & 72.23 & 79.90 & 109.90 \\
\hline \multicolumn{7}{|c|}{$R\left(K_{D P}, Z_{D R}\right)=a\left|K_{D P}\right|^{b} Z_{d r}^{c} \operatorname{sign}\left(K_{D P}\right)$} \\
\hline 17 & 218.90 & 70.90 & 103.01 & 205.86 & 332.39 & 424.72 \\
\hline 18 & 288.30 & 82.58 & 122.98 & 276.08 & 437.23 & 579.75 \\
\hline 19 & 154.48 & 58.89 & 89.92 & 140.89 & 230.82 & 284.59 \\
\hline 20 & 182.50 & 66.82 & 94.35 & 172.06 & 275.67 & 340.27 \\
\hline \multicolumn{7}{|c|}{ Synthetic } \\
\hline 21 & 87.23 & 46.99 & 68.11 & 89.89 & 102.03 & 121.47 \\
\hline
\end{tabular}


Table 3.14.2 Trace Techniques, FRMSE (\%) by Percentile

\begin{tabular}{|c|c|c|c|c|c|c|c|}
\hline & $Z_{D R}+$ & Mean & $0^{\text {th }}$ & $25^{\text {th }}$ & $50^{\text {th }}$ & $75^{\text {th }}$ & $100^{\text {th }}$ \\
\hline \multirow[t]{14}{*}{$\mathrm{T} 1$} & 0 & 116.27 & 26.55 & 70.06 & 90.36 & 141.65 & 280.14 \\
\hline & 0.2 & 98.95 & 26.04 & 62.34 & 77.51 & 106.03 & 246.03 \\
\hline & 0.3 & 91.80 & 26.08 & 60.26 & 73.98 & 97.95 & 230.79 \\
\hline & 0.4 & 85.48 & 26.24 & 58.37 & 67.84 & 93.27 & 216.01 \\
\hline & 0.5 & 80.14 & 26.66 & 54.27 & 64.53 & 88.76 & 202.74 \\
\hline & 0.6 & 75.59 & 27.47 & 48.64 & 61.93 & 83.27 & 189.31 \\
\hline & 0.7 & 71.80 & 28.64 & 46.30 & 59.01 & 80.67 & 176.80 \\
\hline & 0.8 & 68.95 & 31.05 & 44.97 & 58.05 & 79.76 & 163.24 \\
\hline & 0.9 & 66.99 & 33.26 & 45.98 & 57.49 & 80.03 & 152.39 \\
\hline & 1 & 65.79 & 35.70 & 48.14 & 56.52 & 80.59 & 141.86 \\
\hline & 1.1 & 65.09 & 36.99 & 50.76 & 56.70 & 74.18 & 133.26 \\
\hline & 1.5 & 66.33 & 40.57 & 54.05 & 63.53 & 78.66 & 106.70 \\
\hline & 2 & 72.19 & 49.31 & 58.48 & 70.00 & 78.15 & 121.15 \\
\hline & 2.6 & 81.43 & 62.30 & 70.42 & 78.23 & 88.12 & 132.78 \\
\hline \multirow[t]{12}{*}{ T2 } & 0.4 & 168.05 & 67.04 & 126.62 & 141.85 & 218.39 & 313.31 \\
\hline & 0.6 & 138.02 & 47.45 & 105.88 & 120.82 & 178.91 & 240.54 \\
\hline & 0.8 & 116.18 & 43.01 & 92.15 & 114.92 & 147.07 & 183.49 \\
\hline & 1 & 99.93 & 47.21 & 80.05 & 102.71 & 122.56 & 138.47 \\
\hline & 1.2 & 89.51 & 48.76 & 69.72 & 94.88 & 105.79 & 121.12 \\
\hline & 1.4 & 82.99 & 49.01 & 64.15 & 82.62 & 95.44 & 136.40 \\
\hline & 1.6 & 79.50 & 53.38 & 62.12 & 72.68 & 88.61 & 157.11 \\
\hline & 1.8 & 78.19 & 48.80 & 59.32 & 72.92 & 79.71 & 178.50 \\
\hline & 2 & 78.22 & 41.45 & 57.59 & 70.01 & 77.73 & 188.82 \\
\hline & 2.2 & 79.19 & 39.10 & 58.02 & 70.50 & 80.79 & 184.66 \\
\hline & 2.4 & 81.59 & 40.60 & 60.63 & 72.67 & 84.88 & 183.84 \\
\hline & 2.6 & 84.74 & 44.35 & 64.67 & 74.83 & 88.78 & 186.22 \\
\hline \multirow[t]{14}{*}{ T3 } & 0 & 110.08 & 28.56 & 64.50 & 92.27 & 129.79 & 247.34 \\
\hline & 0.2 & 94.74 & 29.87 & 56.59 & 81.70 & 106.57 & 213.18 \\
\hline & 0.3 & 88.01 & 31.34 & 54.28 & 74.56 & 100.41 & 197.15 \\
\hline & 0.4 & 82.13 & 33.18 & 51.35 & 71.65 & 95.22 & 182.85 \\
\hline & 0.5 & 77.02 & 34.41 & 46.92 & 66.90 & 89.99 & 167.65 \\
\hline & 0.6 & 72.76 & 35.78 & 46.10 & 62.61 & 85.93 & 153.18 \\
\hline & 0.7 & 69.47 & 36.61 & 46.27 & 59.26 & 82.75 & 139.18 \\
\hline & 0.8 & 66.76 & 37.98 & 45.60 & 58.42 & 80.20 & 126.51 \\
\hline & 0.9 & 64.84 & 40.76 & 43.57 & 58.37 & 78.52 & 115.77 \\
\hline & 1 & 63.72 & 40.97 & 44.46 & 58.95 & 78.01 & 107.13 \\
\hline & 1.1 & 62.92 & 40.77 & 45.12 & 60.00 & 76.85 & 98.64 \\
\hline & 1.5 & 64.26 & 42.92 & 52.74 & 65.72 & 73.52 & 90.70 \\
\hline & 2 & 72.44 & 53.12 & 58.23 & 72.01 & 79.99 & 117.52 \\
\hline & 2.6 & 83.76 & 66.67 & 68.97 & 81.02 & 92.06 & 122.59 \\
\hline
\end{tabular}


Table 3.14.2 Trace Techniques, FRMSE (\%) by Percentile (Continued)

\begin{tabular}{|c|c|c|c|c|c|c|c|}
\hline & $Z_{D R}+$ & Mean & $0^{\text {th }}$ & $25^{\text {th }}$ & $50^{\text {th }}$ & $75^{\text {th }}$ & $100^{\text {th }}$ \\
\hline \multirow[t]{12}{*}{ T4 } & 0.4 & 155.85 & 63.35 & 118.49 & 139.72 & 206.84 & 265.93 \\
\hline & 0.6 & 128.13 & 42.86 & 104.71 & 114.51 & 169.34 & 207.18 \\
\hline & 0.8 & 107.76 & 38.63 & 87.39 & 106.53 & 139.03 & 157.46 \\
\hline & 1 & 93.20 & 43.81 & 73.03 & 99.13 & 116.17 & 131.62 \\
\hline & 1.2 & 83.73 & 46.76 & 63.12 & 87.91 & 98.29 & 118.07 \\
\hline & 1.4 & 77.69 & 46.93 & 60.36 & 73.95 & 87.54 & 129.13 \\
\hline & 1.6 & 74.47 & 51.59 & 57.34 & 66.29 & 83.18 & 143.07 \\
\hline & 1.8 & 73.61 & 46.29 & 57.44 & 67.26 & 72.70 & 159.95 \\
\hline & 2 & 74.51 & 42.06 & 57.42 & 65.43 & 75.61 & 169.13 \\
\hline & 2.2 & 76.27 & 42.13 & 57.58 & 66.85 & 79.91 & 168.37 \\
\hline & 2.4 & 79.13 & 45.00 & 57.28 & 72.01 & 84.05 & 168.13 \\
\hline & 2.6 & 82.63 & 49.29 & 61.20 & 76.36 & 88.07 & 170.89 \\
\hline \multirow[t]{12}{*}{ T5 } & 0.4 & 164.54 & 72.74 & 100.17 & 149.78 & 232.19 & 283.99 \\
\hline & 0.6 & 136.50 & 51.97 & 92.66 & 118.66 & 191.06 & 228.09 \\
\hline & 0.8 & 115.38 & 44.07 & 84.14 & 104.06 & 157.50 & 188.50 \\
\hline & 1 & 99.92 & 45.73 & 71.61 & 103.32 & 133.77 & 154.28 \\
\hline & 1.2 & 90.20 & 51.51 & 58.96 & 86.13 & 118.54 & 135.90 \\
\hline & 1.4 & 84.53 & 47.38 & 59.54 & 81.78 & 112.68 & 132.23 \\
\hline & 1.6 & 80.33 & 43.37 & 54.65 & 75.36 & 100.96 & 151.63 \\
\hline & 1.8 & 78.15 & 41.61 & 54.16 & 71.73 & 91.64 & 169.96 \\
\hline & 2 & 77.10 & 39.87 & 53.50 & 70.35 & 83.44 & 179.51 \\
\hline & 2.2 & 77.24 & 41.93 & 51.93 & 67.44 & 80.67 & 183.22 \\
\hline & 2.4 & 78.91 & 46.17 & 55.77 & 68.59 & 82.61 & 188.80 \\
\hline & 2.6 & 81.44 & 45.27 & 59.88 & 69.51 & 86.31 & 193.16 \\
\hline
\end{tabular}




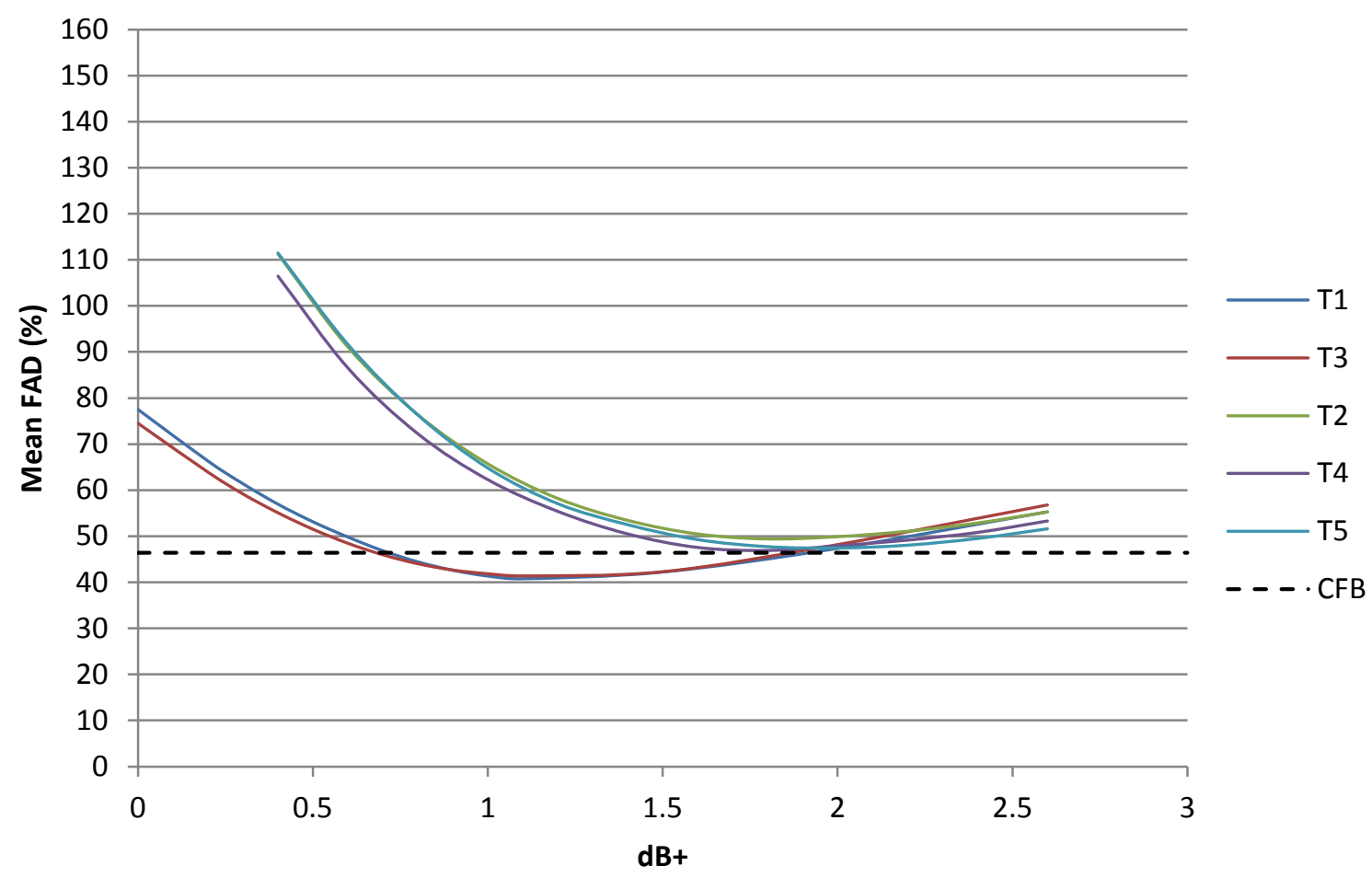

Figure 3.13.1. Mean $F A D$ for the five trace techniques as a function of $Z_{D R}$ adjustment.

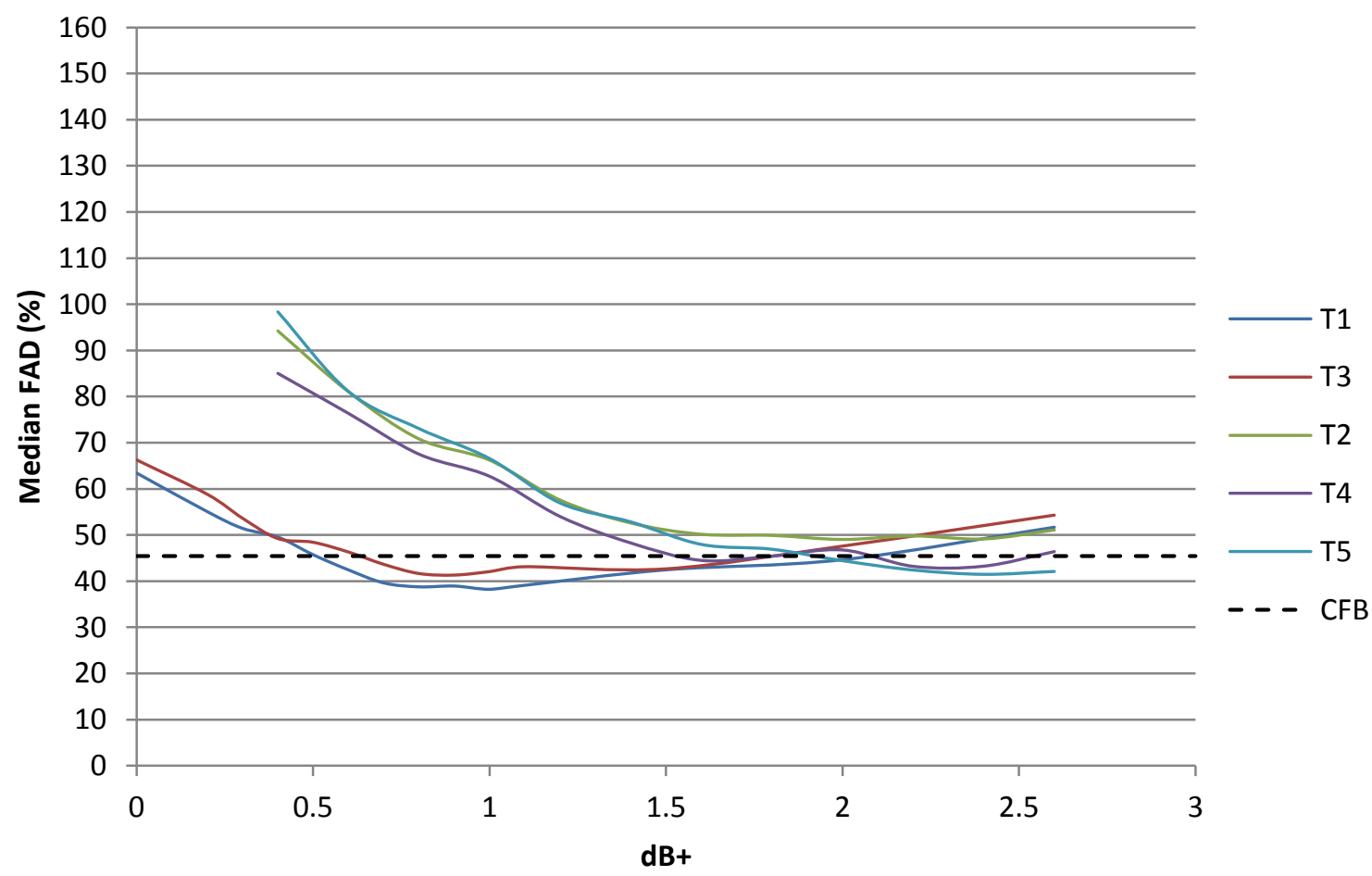

Figure 3.13.2. Median FAD for the five trace techniques as a function of $Z_{D R}$ adjustment. 


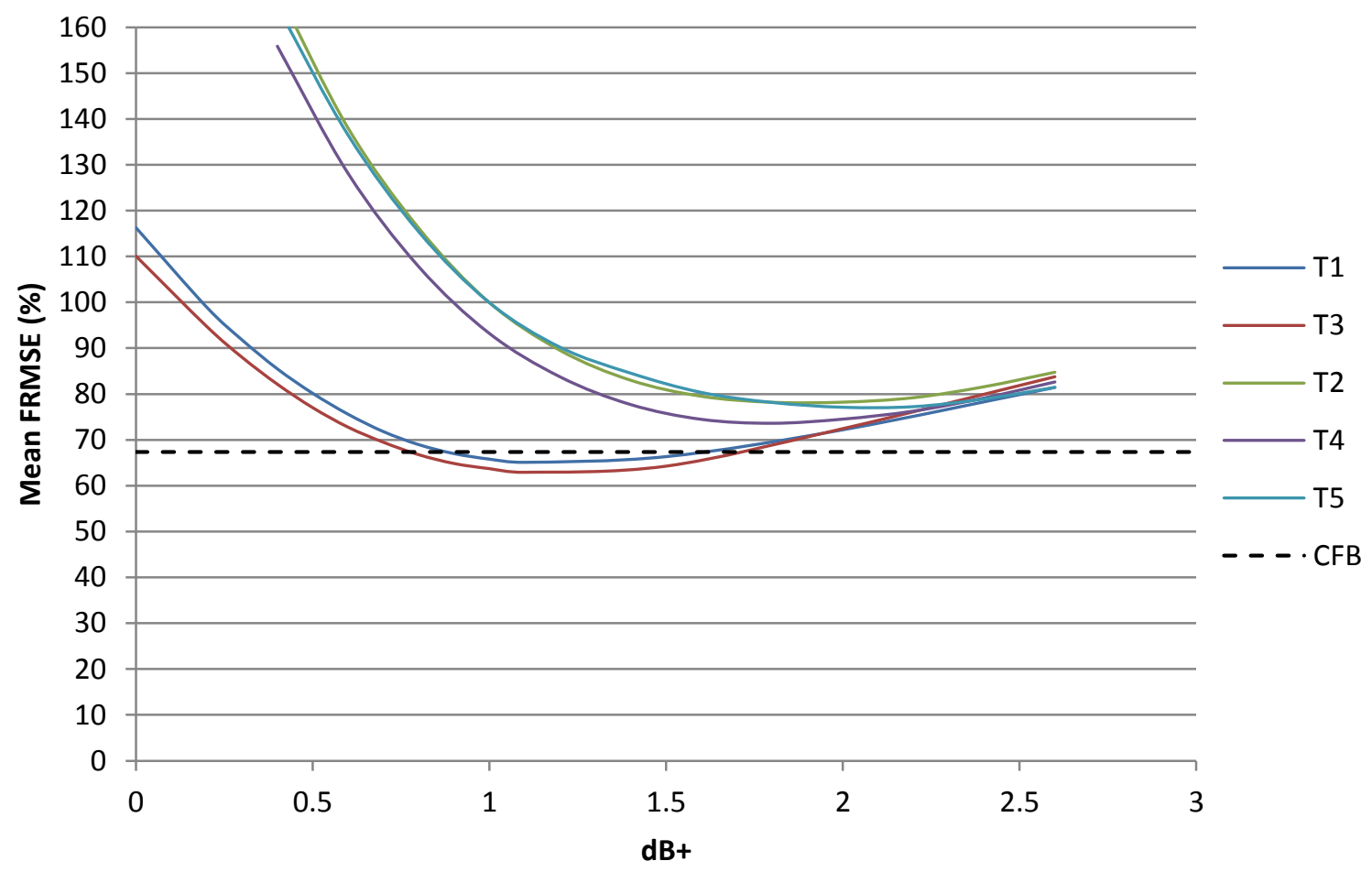

Figure 3.13.3. Mean FRMSE for the five trace techniques as a function of $Z_{D R}$ adjustment.

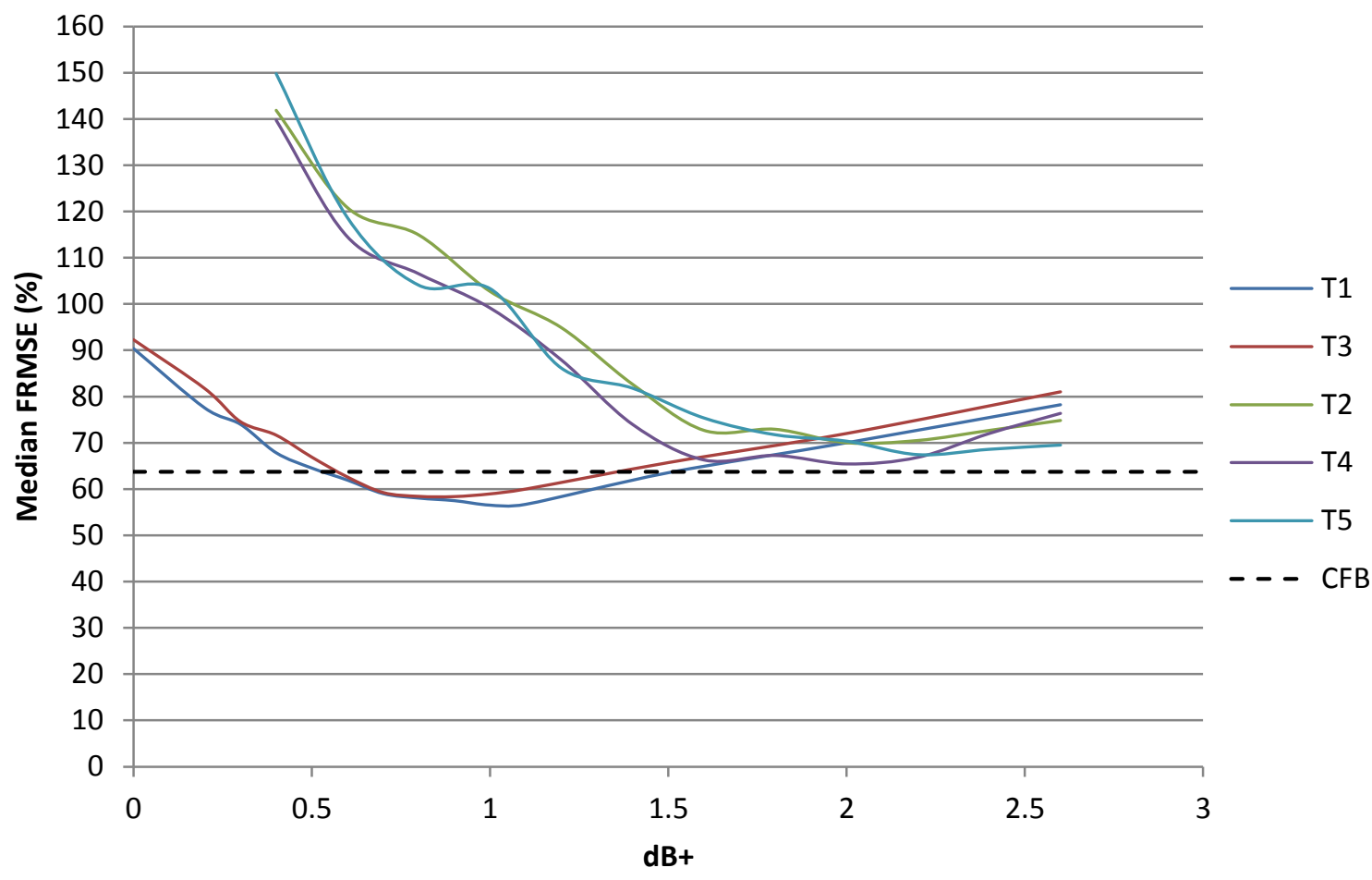

Figure 3.13.4. Median FRMSE for the five trace techniques as a function of $Z_{D R}$ adjustment. 


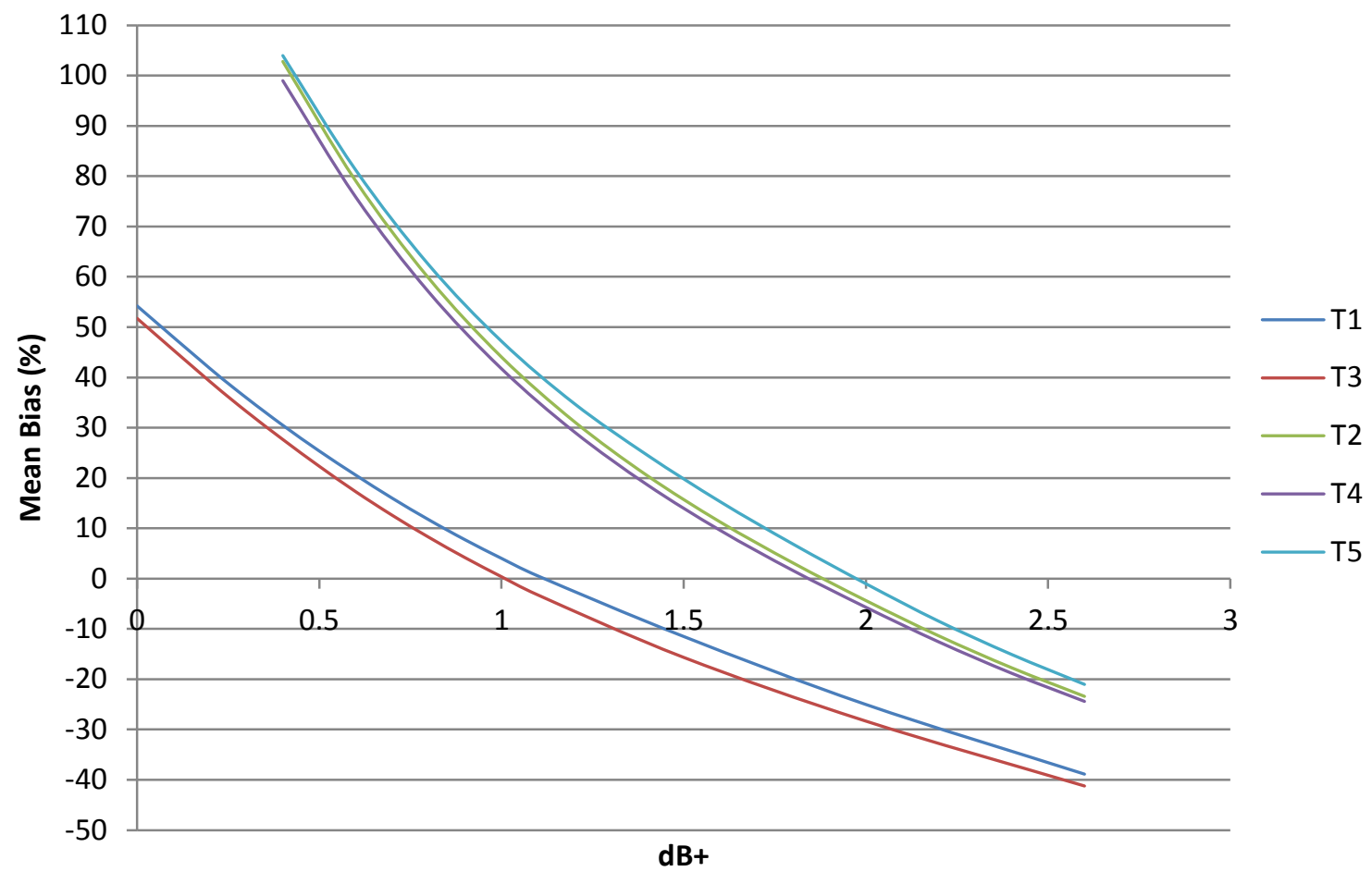

Figure 3.13.5. Mean bias for the five trace techniques as a function of $Z_{D R}$ adjustment.

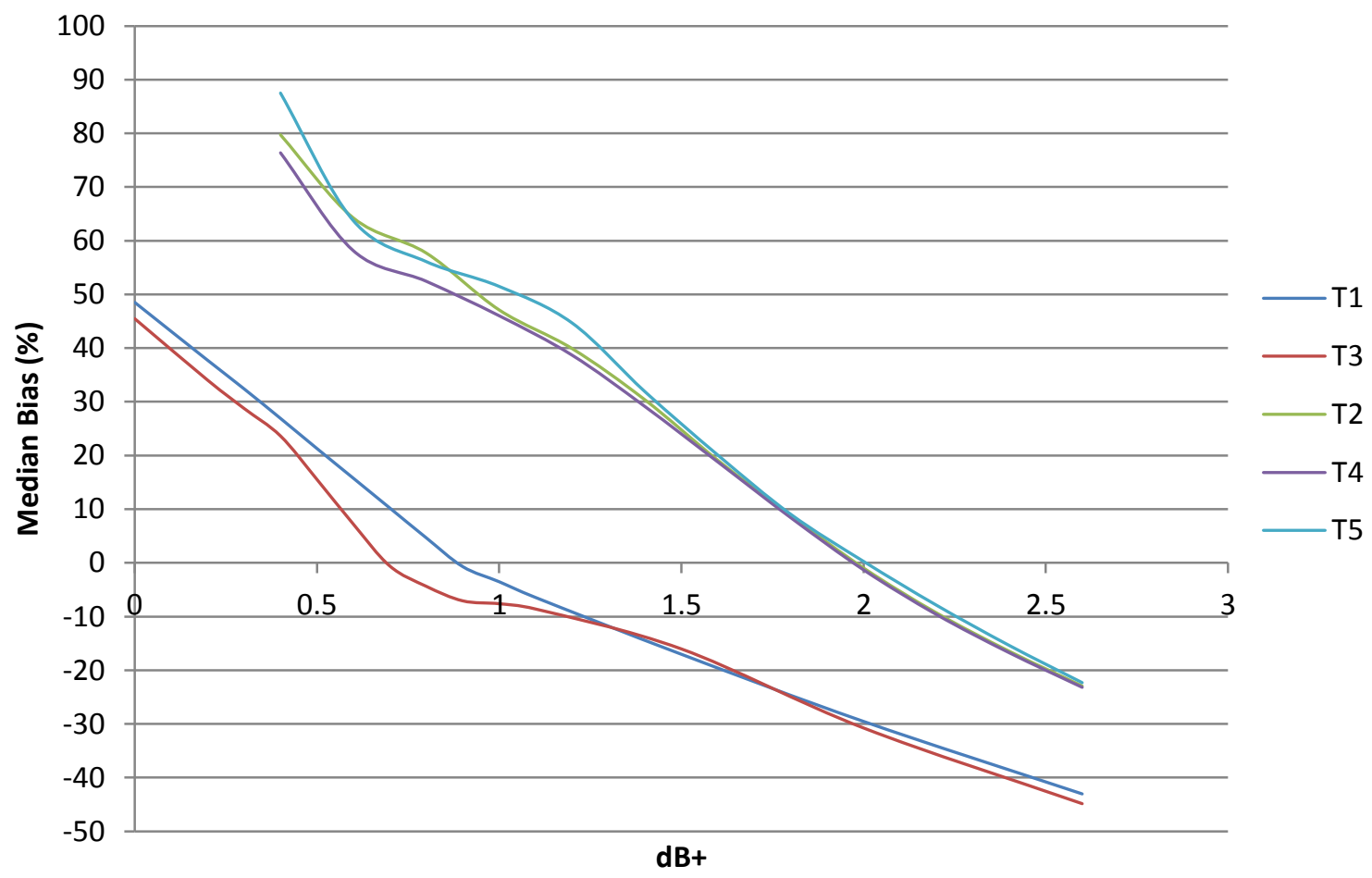

Figure 3.13.6. Median bias for the five trace techniques as a function of $Z_{D R}$ adjustment. 


\section{Chapter 4. Conclusions}

Two primary objectives were discussed in the introduction and methodology. The first objective was to determine the most effective radar rainfall estimation technique for locations in Central Missouri. The second objective was to assess whether the introduction of high resolution model data could be used to improve radar rainfall estimation.

The optimal T1 and T3 trace techniques performed best overall given the assumptions in the study. T1 accounted for evaporation and precipitation drift, while T3 only accounted for evaporation. The mean overall FAD for the $+1.1 Z_{D R} T 1$ technique was $40.76 \%$, while the mean overall FAD for the $+1.1 Z_{D R} T 3$ technique was $41.38 \%$. The best mean overall FAD performance for the conventional formulas was a tie between (1) and (4) at $46.41 \%$. This comes with the caveat that the $K_{D P}$ data was extremely noisy, which likely degraded the performance of the formulas that rely on it significantly.

Median FAD values were similar to mean FAD values. The best performer with respect to median FAD was the $+1.0 Z_{D R} T 1$ technique at $38.23 \%$. The optimal $\mathrm{T} 3$ technique $(+0.9$ $Z_{D R}$ ) performed slightly worse at $41.32 \%$. The best conventional technique was (4) at $45.43 \%$. 
With respect to FRMSE, the mean overall FRMSE for the $+1.1 \mathrm{Z}_{\mathrm{DR}} \mathrm{T} 1$ technique was $65.09 \%$, while the mean overall FRMSE for the $+1.1 Z_{D R} T 3$ technique was $62.92 \%$. The best mean overall FRMSE performance for the conventional formulas was (1) at $67.35 \%$.

Median FRMSE values were moderately lower than mean FRMSE values, likely due to the outlier penalty association with the FRMSE evaluation parameter. The top performer with respect to median FRMSE was the $+1.0 \mathrm{Z}_{\mathrm{DR}} \mathrm{T} 1$ technique at $56.52 \%$. The optimal T3 technique $\left(+0.9 \mathrm{Z}_{\mathrm{DR}}\right)$ was again slightly worse at $58.37 \%$. The best of the conventional formulas with respect to median FRMSE was (4) at $63.73 \%$.

The FRMSE results obtained in this study did not differ significantly from those found in RGS05 (optimal FRMSE in the $\sim 50 \%$ range). The comparison is not exact for a variety of reasons. The gauge locations for this study were much further from the radar than in RGS05. The rainfall events were different, and the process in which the data from differing events was combined was different. One point of interest is the relative performance of the formulas dependent on just $Z$ and those dependent on combinations of $Z, Z_{D R}$, and $K_{D P}$. In RGS05 the formulas using the polarimetric parameters performed slightly better than those just using $Z$, while the opposite was the case for this study. One possible cause may be range from the radar affecting the quality of the $Z_{D R}$ data. The reason may also involve the problems with the $K_{D P}$ data described in Chapter 2.

Qualitative analysis of Figures 3.13.1-3.13.6 reveals a considerable difference between the $\mathrm{T} 1$ and $\mathrm{T} 3$ trace techniques and the $\mathrm{T} 2, \mathrm{~T} 4$, and $\mathrm{T} 5$ techniques. The minimum values 
for FAD and FRMSE are reached at a difference of roughly $1 \mathrm{~dB}$ in $\mathrm{Z}_{\mathrm{DR}}$ adjustment between the two sets. This is convincing evidence that evaporation plays a significant role in radar rainfall estimation and cannot be ignored (at least for locations far from the radar). This also makes the case for inclusion of high resolution model data into radar rainfall estimation, as calculating evaporation would be impossible without the model data.

Much less clear is the effect of precipitation drift on radar rainfall estimation. For some events it provided performance improvement, but for others it detracted from performance. This may be related to the beam broadening source of uncertainty discussed in the introduction. Studies from Chapter 1 indicated that the effects of precipitation drift would be most noticeable in high resolution situations. At the distance Columbia is from the radar, the resolution was probably a significant factor. As noted in Section 3.13, a minor improvement in performance was seen when the time it took for precipitation to reach the ground was accounted for. This is an area where further investigation is warranted, particularly for finer timescales. 


\section{Chapter 5. Recommendations}

\section{1 $Z_{D R}$ Adjustment}

Several areas for further research with regard to the conventional formulas are noteworthy. The formulas reliant on differential reflectivity may see some improvement from $Z_{D R}$ adjustment. Limited experimentation was done using various $Z_{D R}$ alterations, but the results proved inconclusive. Furthermore, the formulas reliant on $Z_{D R}$ generally exhibited negative biases, meaning a reduction in $Z_{D R}$ would be necessary. This is the opposite direction from which the trace techniques saw improvement, and would not be physically expected, considering the already large number of negative $Z_{D R}$ values.

\subsection{Filtering}

$K_{D P}$ is an area for further investigation. Some of the extreme noise seen in the $K_{D P}$ data may have been the result of problems within the merging process described in Chapter 2. Further investigation into a proper filter is warranted.

$Z_{D R}$ is another area where noise in the data can cause issues. For this project it remained unfiltered due to several concerns. One concern was that filtering the $Z_{D R}$ would cause problems with the hail adjustment algorithm, negatively effecting results. Another issue was that a simple filtering scheme could not be utilized due to $Z_{D R}$ being ratio; an adjustment would have to be made for reflectivity. Time constraints limited the amount 
of complexity for certain parts of the project, and this is definitely an area that could be further investigated.

\subsection{Resolution}

Range degradation was a major issue affecting all techniques tested. Finding out how higher resolution radar data affects the performance of the trace techniques could prove insightful.

Another area where high vertical resolution could prove useful is tracking raindrops as they pass through different scanned elevation angles. Changes in DSD and reflectivity could provide information on what processes are occurring within the storm.

\subsection{Drop Collisions}

The tracing algorithm did not account for raindrop collisions. This is another area for improvement in the tracing scheme.

\subsection{Evaporation Energy and Feedback into High Resolutions Models}

Calculating the total amount of evaporation occurring within an atmospheric layer could prove useful in ways other than rainfall calculations. Total evaporative cooling within a storm could be estimated, and as a consequence the probably of a downburst occurring could also be calculated. This information could be fed back into the high resolution model, further improving the model forecast, as well as future rainfall estimation. 


\subsection{Operational Tracing}

Several hurdles exist with respect to implementing an operational "trace technique" rainfall estimation program. The tracing technique developed in this project calculated drop paths at only nine distinct locations. Any operational program would require much greater spatial coverage. The computational power necessary would be immense. The infrastructure required to import model data in real time would have to be built as well. 


\section{Chapter 6. References}

Andsager, K., K. V. Beard, and N. F. Laird, 1999: Laboratory Measurements of Axis Ratios for Large Raindrops. Journal of the Atmospheric Sciences, 56, 2673-2683.

Auer, A. H., 1994: Hail recognition through the combined use of radar reflectivity and cloud-top temperatures. Monthly Weather Review, 122, 2218-2221.

Beard, K. V., and C. Chuang, 1987: A New Model for the Equilibrium Shape of Raindrops. Journal of the Atmospheric Sciences, 44, 1509-1524.

Best, A. C., 1950: Empirical formulae for the terminal velocity of water drops falling through the atmosphere. Quarterly Journal of the Royal Meteorological Society, 76, 302311.

Boxel, J. H. v., 1997: Numerical model for the fall speed of raindrops in a rainfall simulator. International Workshop on Wind and Water Erosion, Ghen, Belgium, 77-85.

Brandes, E. A., G. Zhang, and J. Vivekanandan, 2002: Experiments in Rainfall Estimation with a Polarimetric Radar in a Subtropical Environment. Journal of Applied Meteorology, 41, 674-685.

- - 2004: Comparison of Polarimetric Radar Drop Size Distribution Retrieval Algorithms. Journal of Atmospheric and Oceanic Technology, 21, 584-598.

Bringi, V. N., and V. Chandrasekar, 2001: Polarimetric Doppler Weather Radar: Principles and Applications. Cambridge University Press.

Dalezios, N. R., and N. Kouwen, 1990: Radar signal interpretation in warm season rainstorms. Nordic hydrology, 21, 47-64.

Foote, G. B., and P. S. Du Toit, 1969: Terminal Velocity of Raindrops Aloft. Journal of Applied Meteorology, 8, 249-253.

Fulton, R. A., J. P. Breidenbach, D. J. Seo, D. A. Miller, and T. O'Bannon, 1998: The WSR88D rainfall algorithm. Weather and Forecasting, 13, 377-395. 
Goddard, J. W. F., K. L. Morgan, A. J. Illingworth, and H. Sauvageot, 1995: Dualwavelength polarisation measurements in precipitation using the CAMRa and Rabelais radars. 196-198.

Gorgucci, E., V. Chandrasekar, V. N. Bringi, and G. Scarchilli, 2002: Estimation of Raindrop Size Distribution Parameters from Polarimetric Radar Measurements. Journal of the Atmospheric Sciences, 59, 2373-2384.

Gossard, E. E., R. O. Strauch, and R. R. Rogers, 1990: Evolution of Dropsize Distributions in Liquid Precipitation Observed by Ground-Based Doppler Radar. Journal of Atmospheric and Oceanic Technology, 7, 815-828.

Gossard, E. E., R. G. Strauch, D. C. Welsh, and S. Y. Matrosov, 1992: Cloud Layers, Particle Identification, and Rain-Rate Profiles from ZRVf Measurements by Clear-Air Doppler Radars. Journal of Atmospheric and Oceanic Technology, 9, 108-119.

Gunn, R., and G. D. Kinzer, 1949: THE TERMINAL VELOCITY OF FALL FOR WATER DROPLETS IN STAGNANT AIR. Journal of Meteorology, 6, 243-248.

Hoban, N. P., J. G. Cunningham, and D. Zittel, 2014: Using Bragg Scatter to Estimate Systematic Differential Reflectivity Biases on Operational WSR-88Ds. 30th Conference on Environmental Information Processing Technologies.

Illingworth, A. J., and T. M. Blackman, 2002: The Need to Represent Raindrop Size Spectra as Normalized Gamma Distributions for the Interpretation of Polarization Radar Observations. Journal of Applied Meteorology, 41, 286-297.

Keenan, T., and Coauthors, 2003: The Sydney 2000 World Weather Research Programme Forecast Demonstration Project: Overview and Current Status. Bulletin of the American Meteorological Society, 84, 1041-1054.

Kinzer, G. D., and R. Gunn, 1951: The evaporation, temperature and thermal relaxationtime of freely falling waterdrops. Journal of Atmospheric Sciences, 8, 71-83.

Kumjian, M. R., and A. V. Ryzhkov, 2010: The Impact of Evaporation on Polarimetric Characteristics of Rain: Theoretical Model and Practical Implications. Journal of Applied Meteorology and Climatology, 49, 1247-1267.

Lack, S. A., 2004: Quantifying the Effect of Wind-drift on Radar-derived Surface Rainfall Estimations, University of Missouri.

Lakshmanan, V., T. Smith, G. Stumpf, and K. Hondl, 2007: The warning decision support system-integrated information. Weather and Forecasting, 22, 596-612. 
Lakshmanan, V., T. Smith, K. Hondl, G. J. Stumpf, and A. Witt, 2006: A real-time, threedimensional, rapidly updating, heterogeneous radar merger technique for reflectivity, velocity, and derived products. Weather and Forecasting, 21, 802-823.

Lauri, T., J. Koistinen, and D. Moisseev, 2011: Advection-Based Adjustment of Radar Measurements. Monthly Weather Review, 140, 1014-1022.

Li, X., and R. C. Srivastava, 2001: An Analytical Solution for Raindrop Evaporation and Its Application to Radar Rainfall Measurements. Journal of Applied Meteorology, 40, 16071616.

Marshall, J. S., and W. M. K. Palmer, 1948: THE DISTRIBUTION OF RAINDROPS WITH SIZE. Journal of Meteorology, 5, 165-166.

Mittermaier, M. P., R. J. Hogan, and A. J. Illingworth, 2004: Using mesoscale model winds for correcting wind-drift errors in radar estimates of surface rainfall. Quarterly Journal of the Royal Meteorological Society, 130, 2105-2123.

Parish, O. O., and T. W. Putnam, 1977: Equations for the Determination of Humidity from Dewpont and Psychometric Data. NASA, Ed.

Rosenfeld, D., D. B. Wolff, and D. Atlas, 1993: General Probability-matched Relations between Radar Reflectivity and Rain Rate. Journal of Applied Meteorology, 32, 50-72.

Ryzhkov, A., and D. Zrnić, 1996: Assessment of Rainfall Measurement That Uses Specific Differential Phase. Journal of Applied Meteorology, 35, 2080-2090.

Ryzhkov, A. V., S. E. Giangrande, and T. J. Schuur, 2005: Rainfall Estimation with a Polarimetric Prototype of WSR-88D. Journal of Applied Meteorology, 44, 502-515.

Sánchez-Diezma, R., I. Zawadzki, and D. Sempere-Torres, 2000: Identification of the bright band through the analysis of volumetric radar data. Journal of geophysical research, 105, 2225-2236.

Tokay, A., A. Kruger, and W. F. Krajewski, 2001: Comparison of Drop Size Distribution Measurements by Impact and Optical Disdrometers. Journal of Applied Meteorology, 40, 2083-2097.

Tracy, C. R., W. r. Welch, and W. P. Porter, 1980: Properties of Air: A Manual for Use in Biophysical Ecology. Third ed.

Ulbrich, C. W., 1983: Natural Variations in the Analytical Form of the Raindrop Size Distribution. Journal of Climate and Applied Meteorology, 22, 1764-1775. 
Uplinger, W. G., 1981: NEW FORMULA FOR RAINDROP TERMINAL VELOCITY. 389-391.

Villarini, G., and W. Krajewski, 2010: Review of the Different Sources of Uncertainty in Single Polarization Radar-Based Estimates of Rainfall. Surv Geophys, 31, 107-129.

Willis, P. T., and P. Tattelman, 1989: Drop-Size Distributions Associated with Intense Rainfall. Journal of Applied Meteorology, 28, 3-15. 


\section{Appendix A. Data Processing}

\section{A.1 Radar Data}

To convert the radar data into text format the ncks tool from the NCO toolbox was utilized, along with the AutoHotkey program. The AutoHotkey program allows the user to deliver input to the computer via code. The following passage contains a sample autohotkey code using the ncks tool as well as comments (in green) describing the functionality.

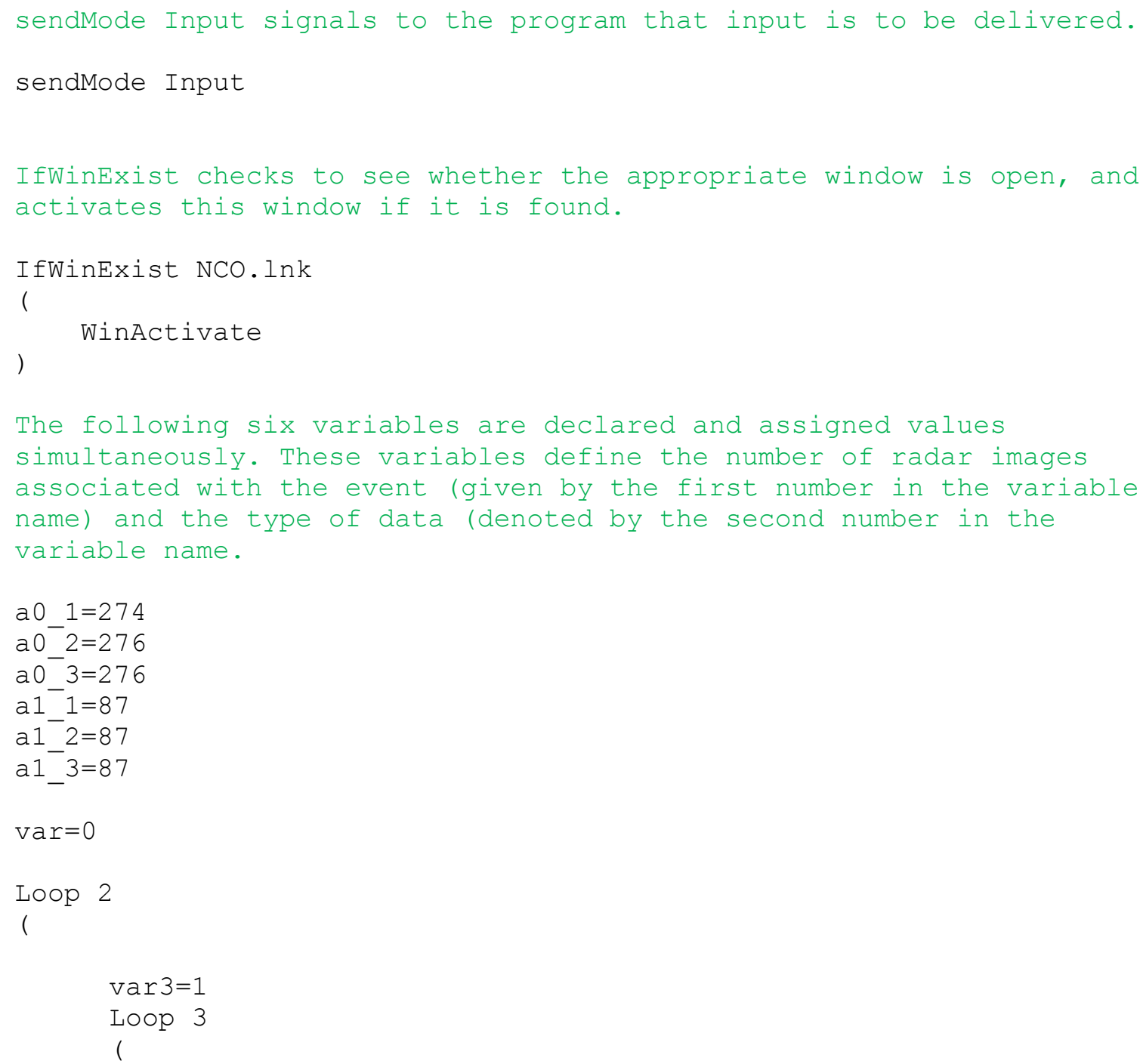




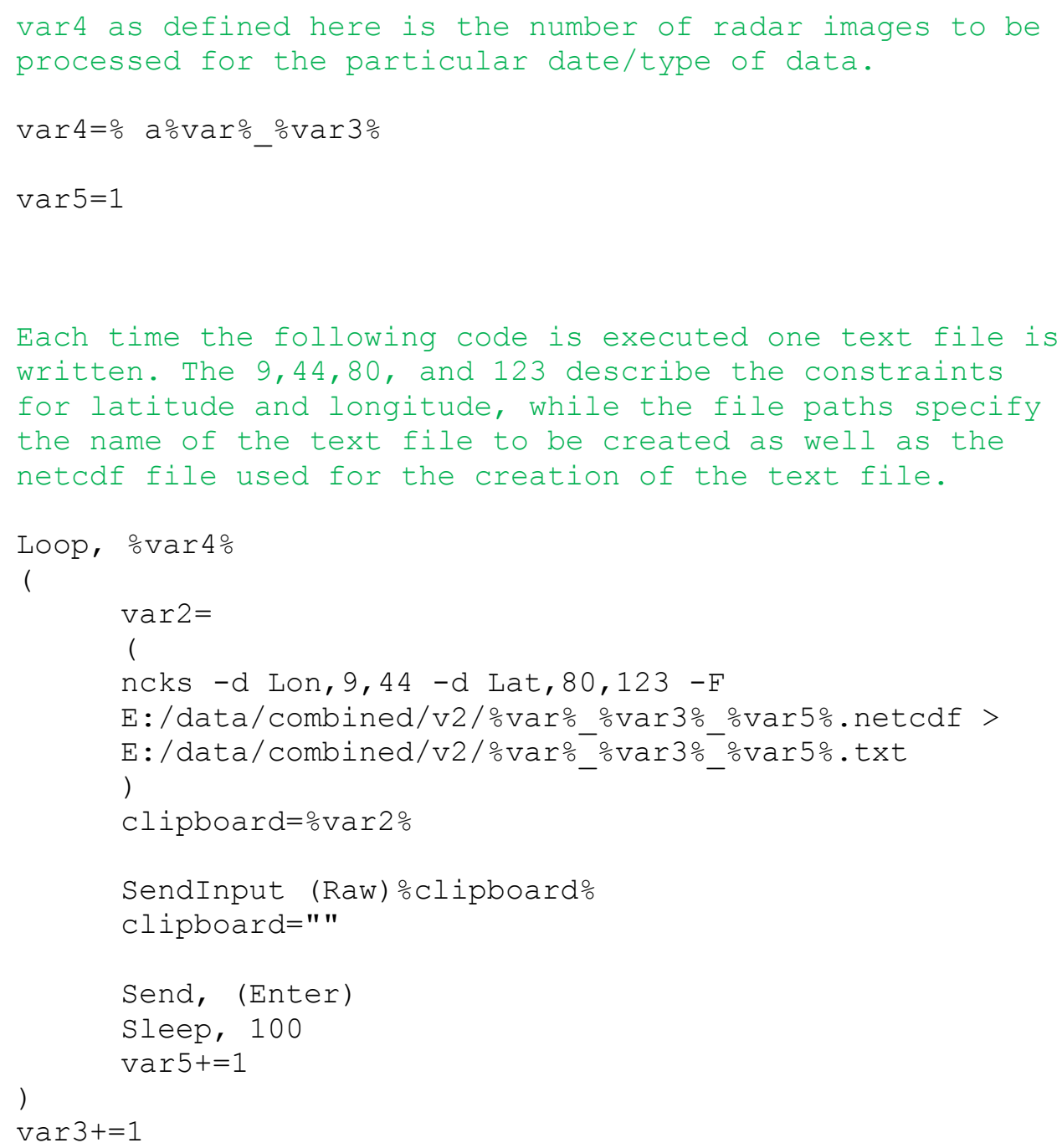




\section{A.2 Model Data}

The wgrib2 tool requires two files to work, the control file and the index file. The

following code describes how these files are created with an autohotkey script using the g2ctl.pl and gribmap tools.

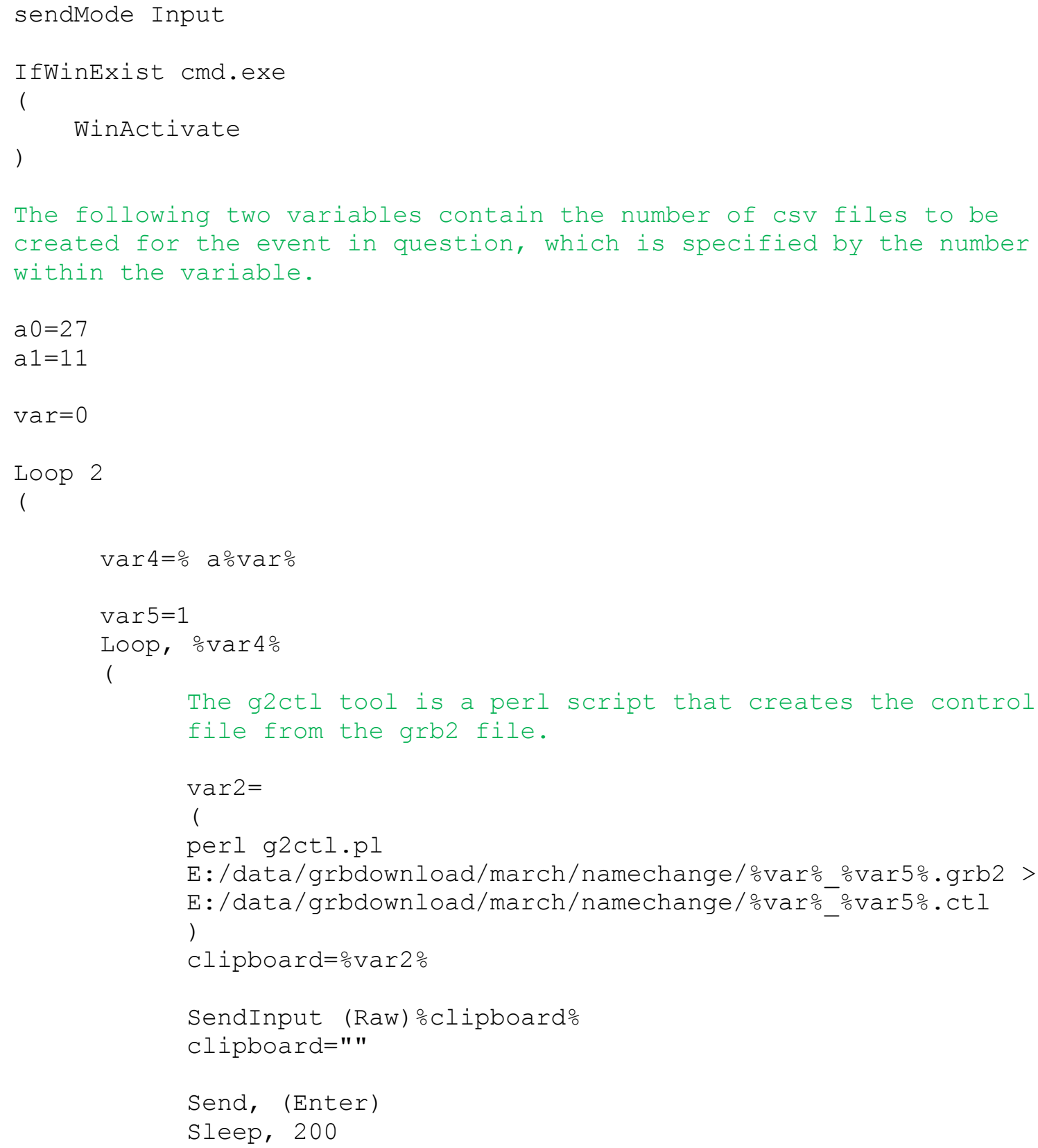




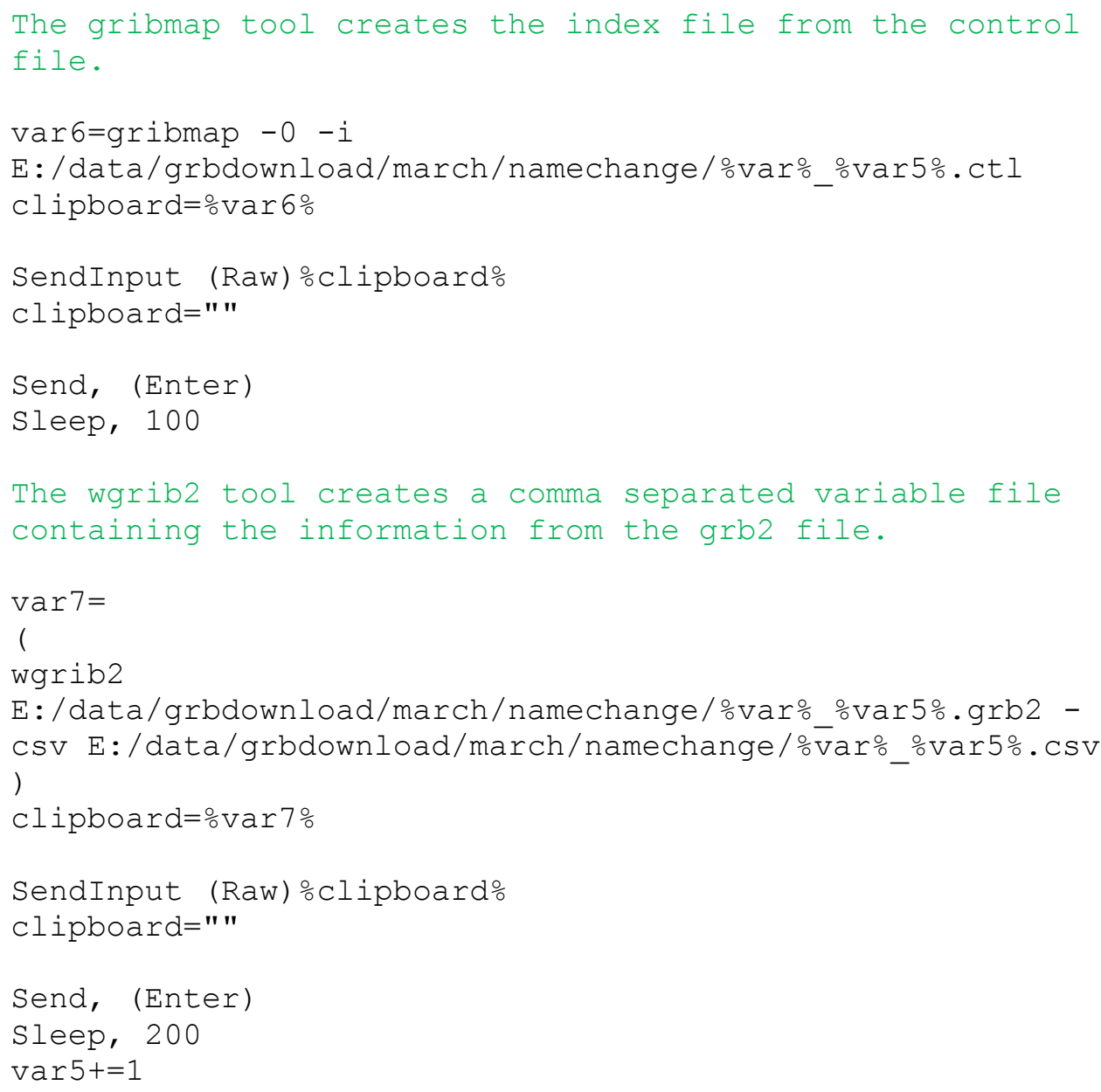




\section{Appendix B. Program Information}

\section{B.1 Program Description}

The VBA program created for this project contains more than 3000 lines of code. Figure B.1 is a simplified flowchart describing how the program functions.

\section{B.2 Hail Adjustment}

A simple algorithm was created for this project to adjust for hail. The hail adjustment algorithm takes reflectivity and differential reflectivity as input, and alters them as described by the following lines of VBA code.

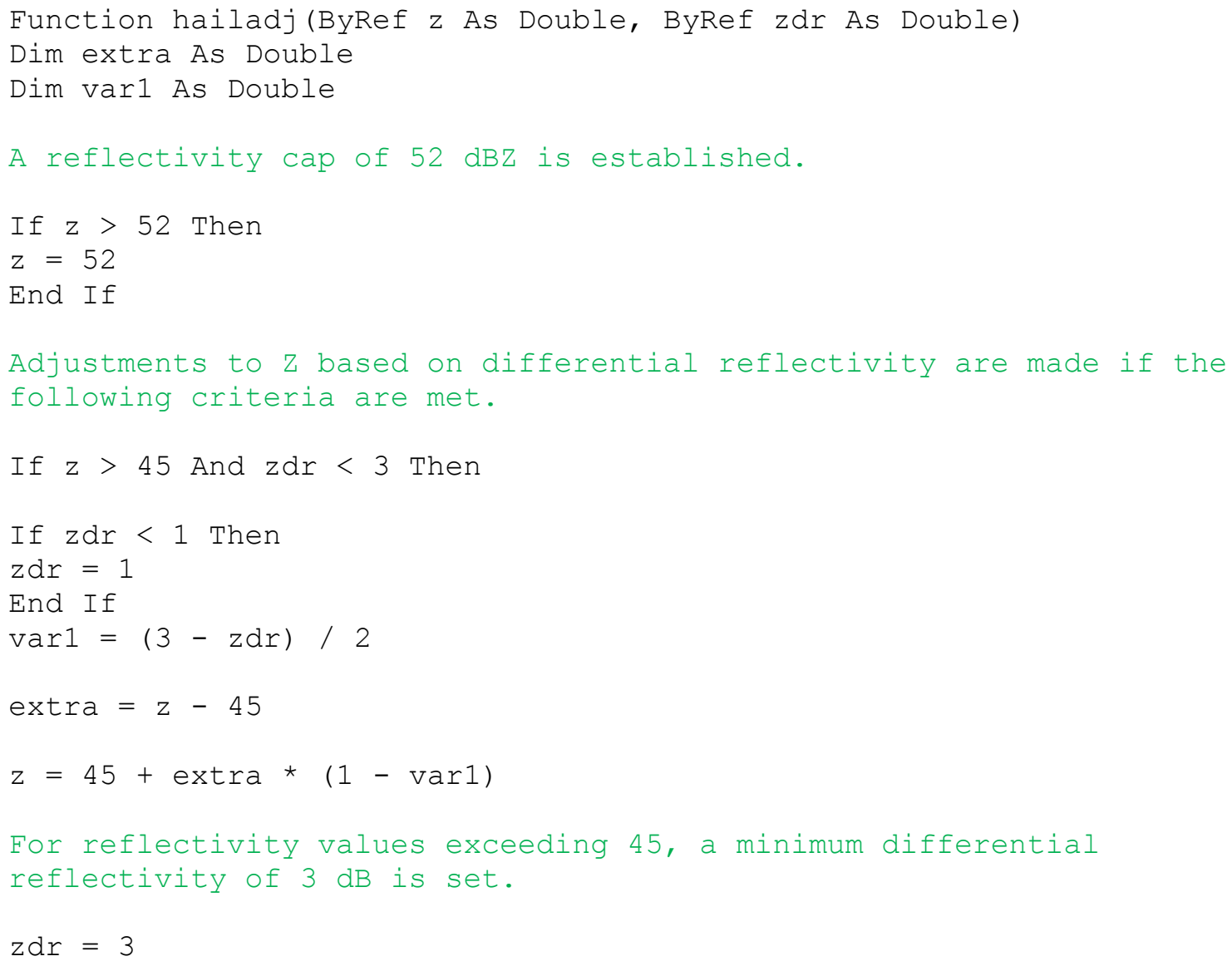


End If

End Function

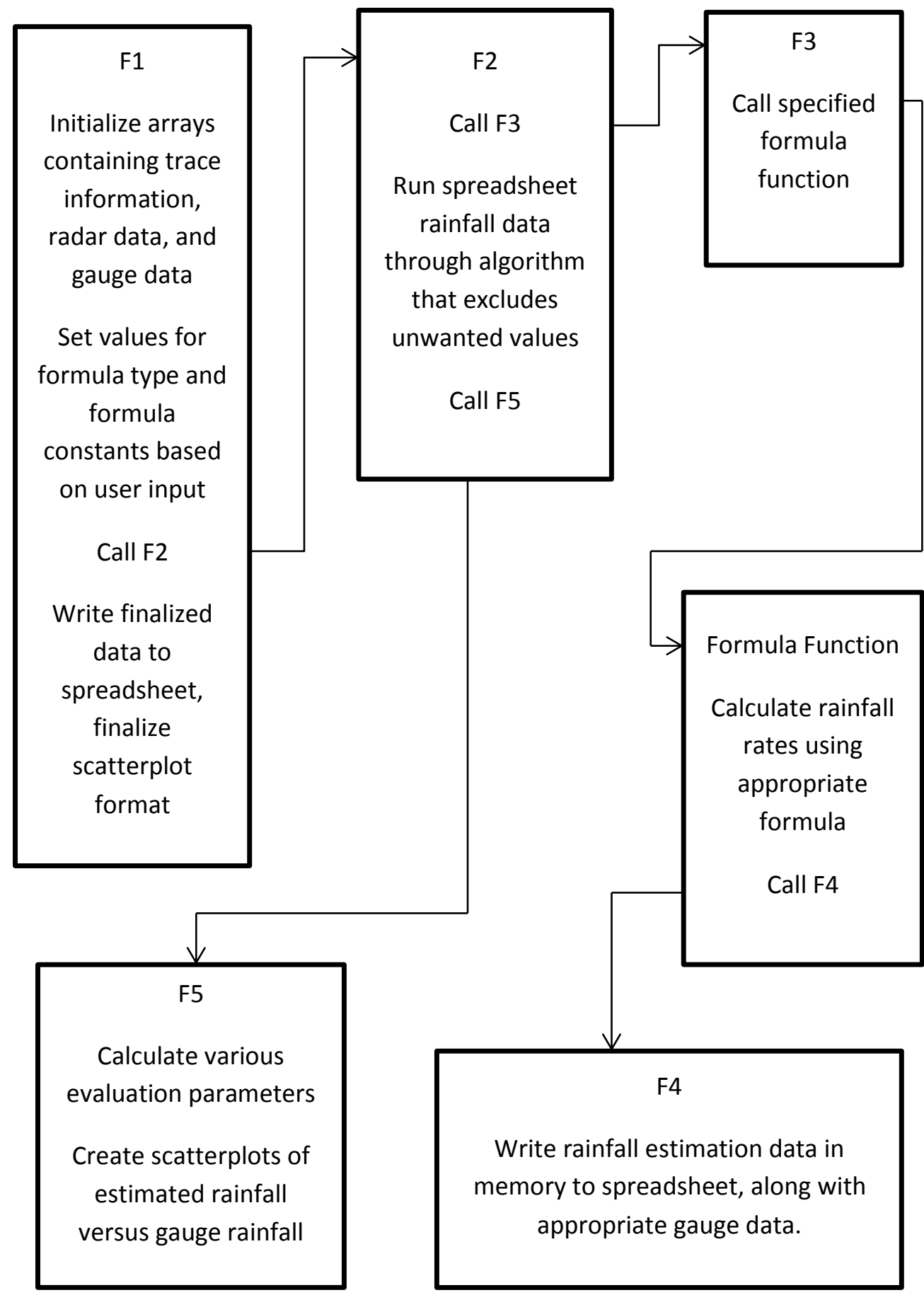

Figure B.1. Program Flowchart. The F1, F2, F3, F4, F5, and Formula Function headers are labels denoting the different routines within the program. 


\section{Appendix C. Trace Information}

\section{C.1 Trace VBA Script}

The tracing of raindrops was done through another VBA script. The following code is applicable for the drift and evaporation trace technique. Appropriate modifications

were made to this script when the other trace techniques were calculated.

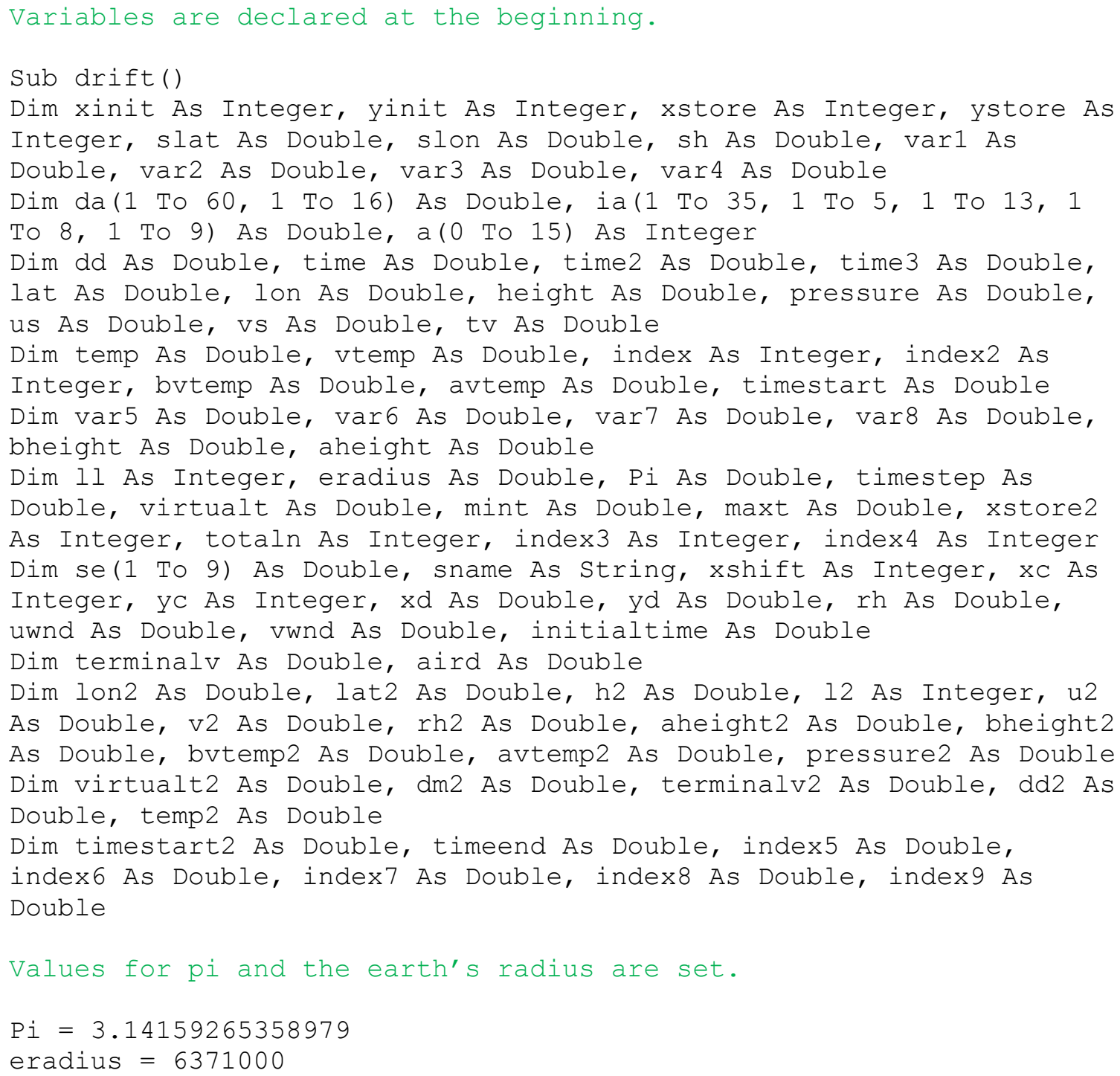


The array a() contains the number of hours of model data stored for each particular event on the spreadsheet.

$a(0)=27$
$a(1)=11$

The se() array contains the elevation of the 9 sites in meters.

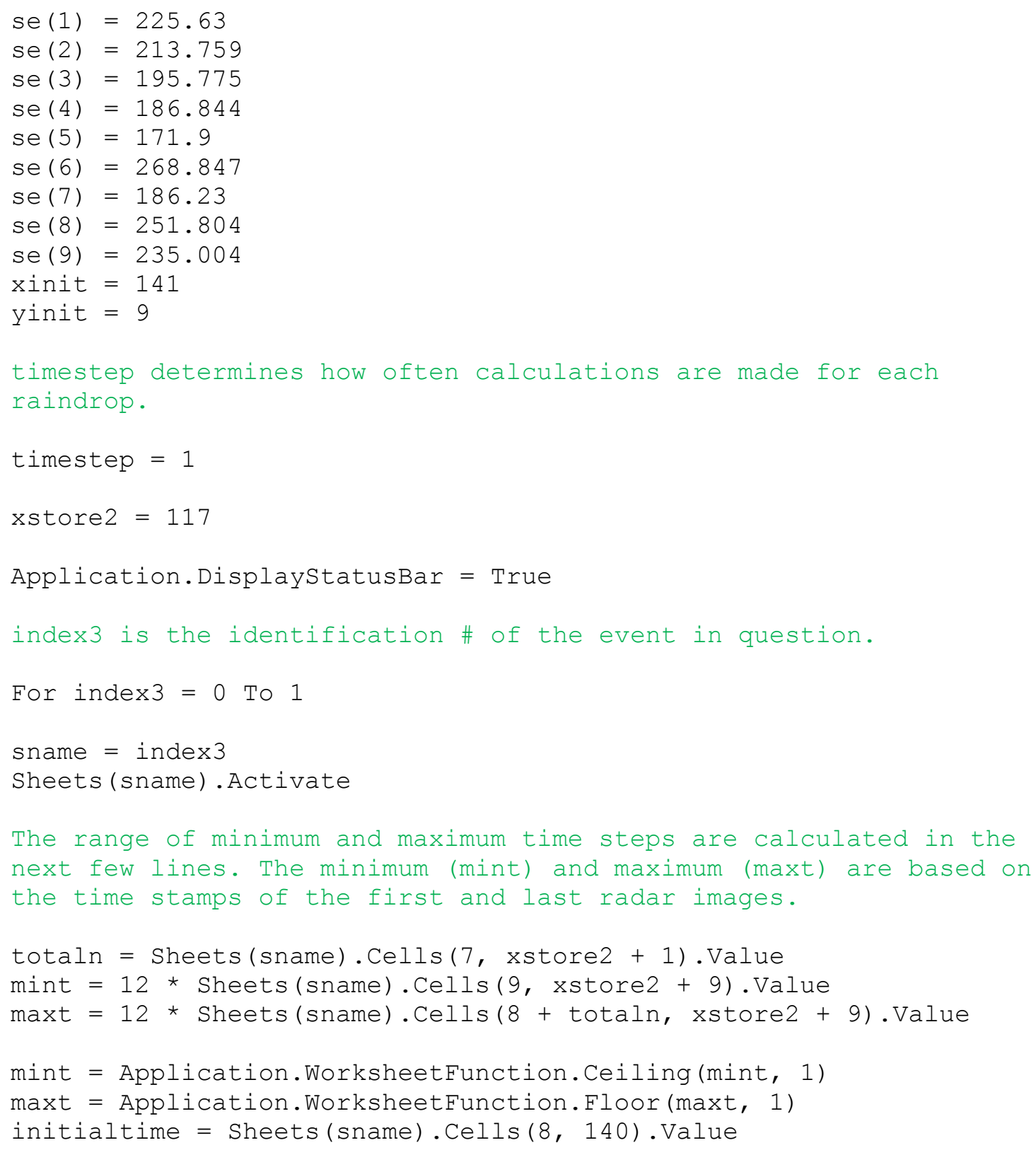

The following "for loops" lift model data stored in the spreadsheet into the program's memory. 


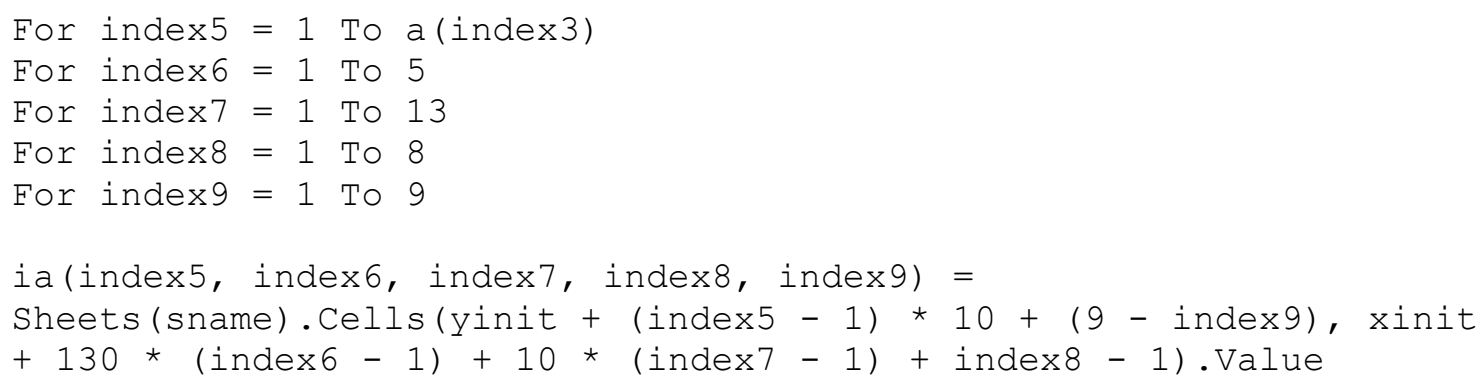




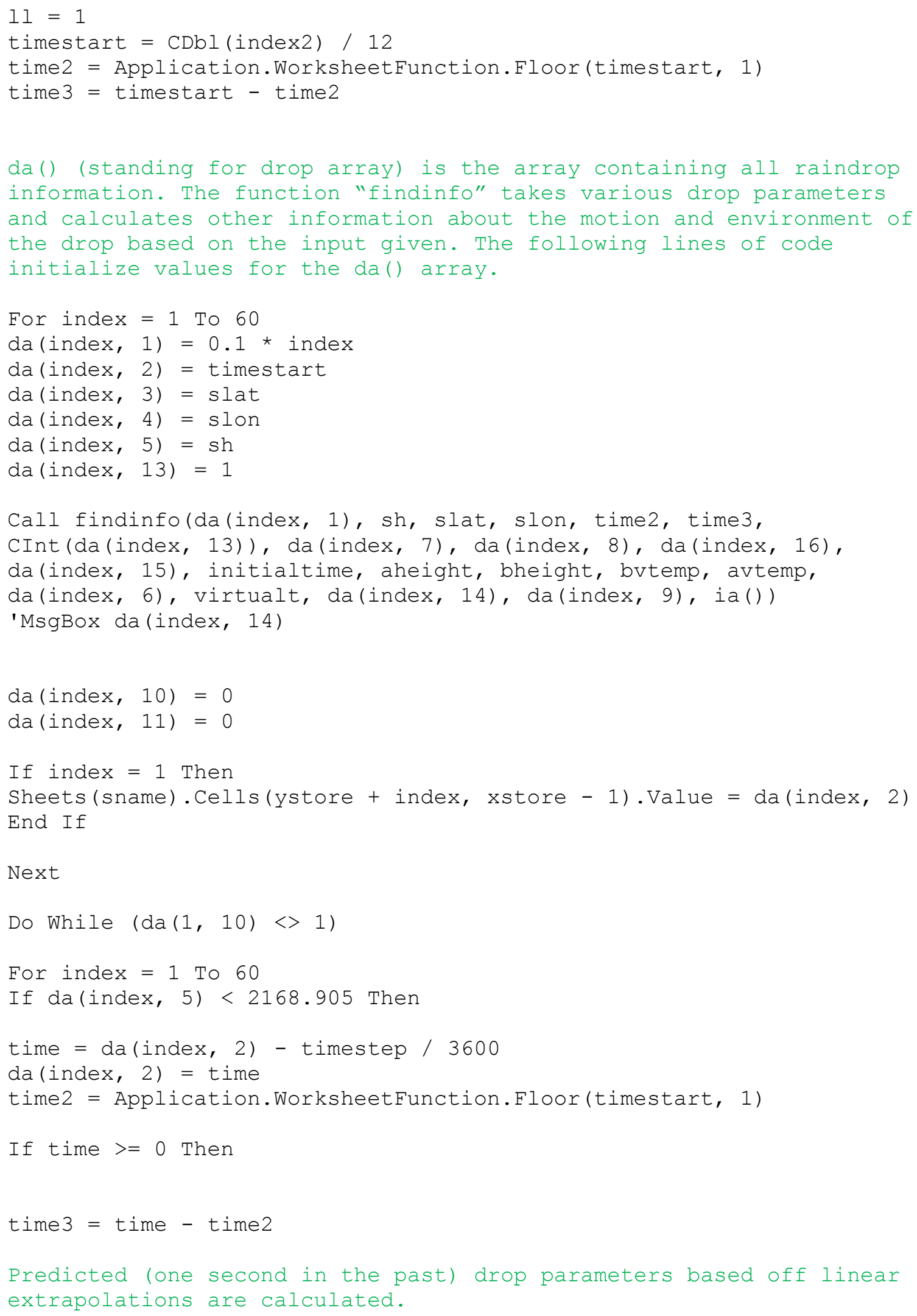




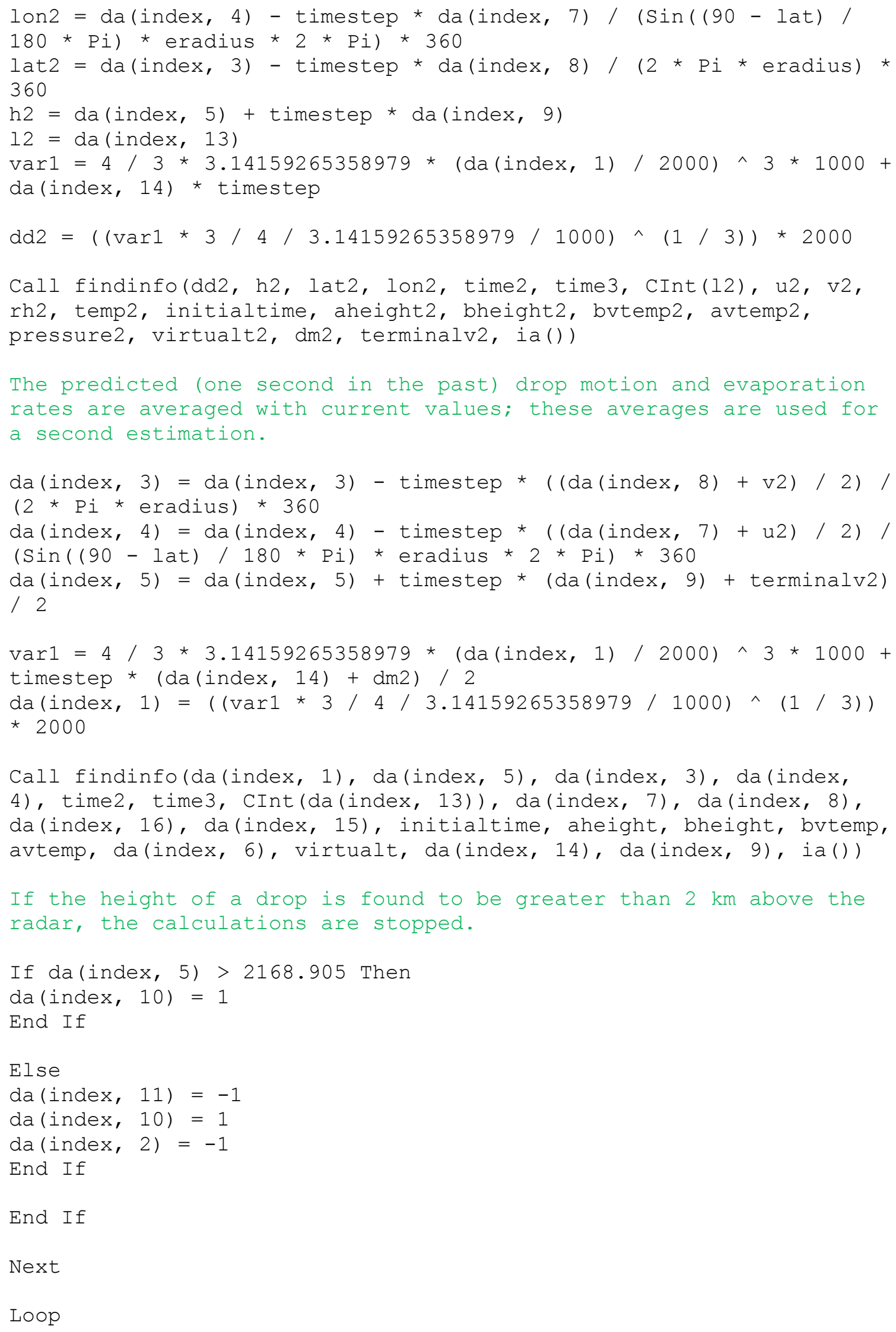




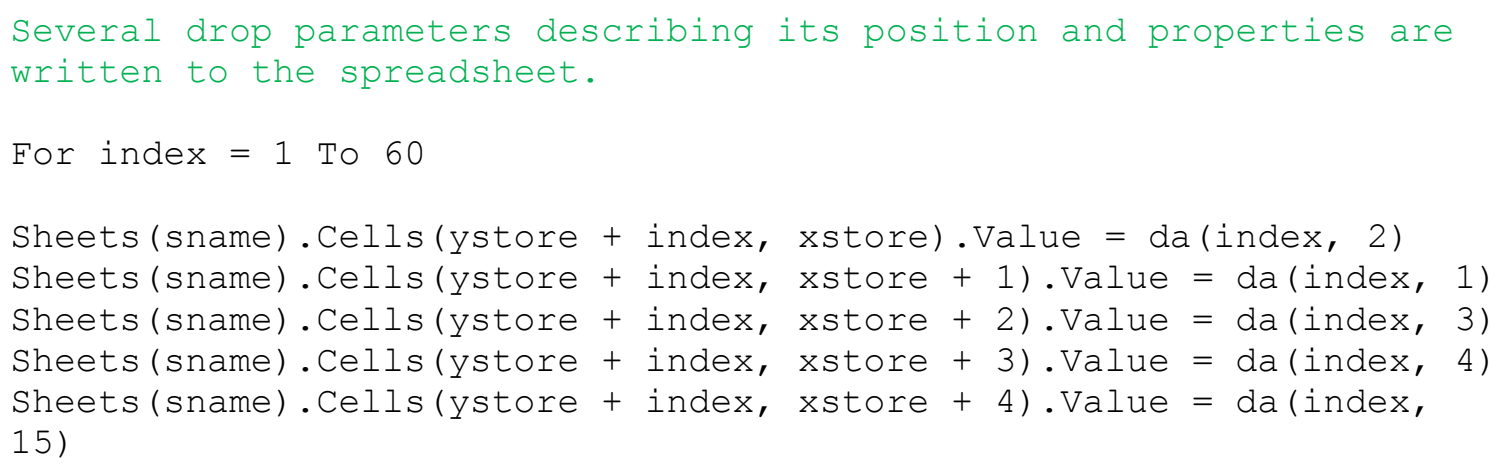

Next

ystore $=$ ystore +61

Next

xstore $=$ xstore +6

Next

Next

End Sub 


\section{C.2 findinfo Script}

The findinfo function was used by the trace program to obtain information about the raindrop in question. The following lines of code describe the findinfo function. Within this function are two more functions called evc() and termvel(). The termvel function determines at what speed the raindrop is falling, while the evc function calculates the evaporation rate. The processes for both of these calculations are described in Chapter 2. The equation used to calculate absolute humidity was obtained from a 1977 NASA Technical Note (Parish and Putnam 1977). The diffusivity of water vapor in air and the kinematic viscosity of air were obtained from a technical report released by researchers from the University of Wisconsin in 1980 (Tracy et al. 1980).

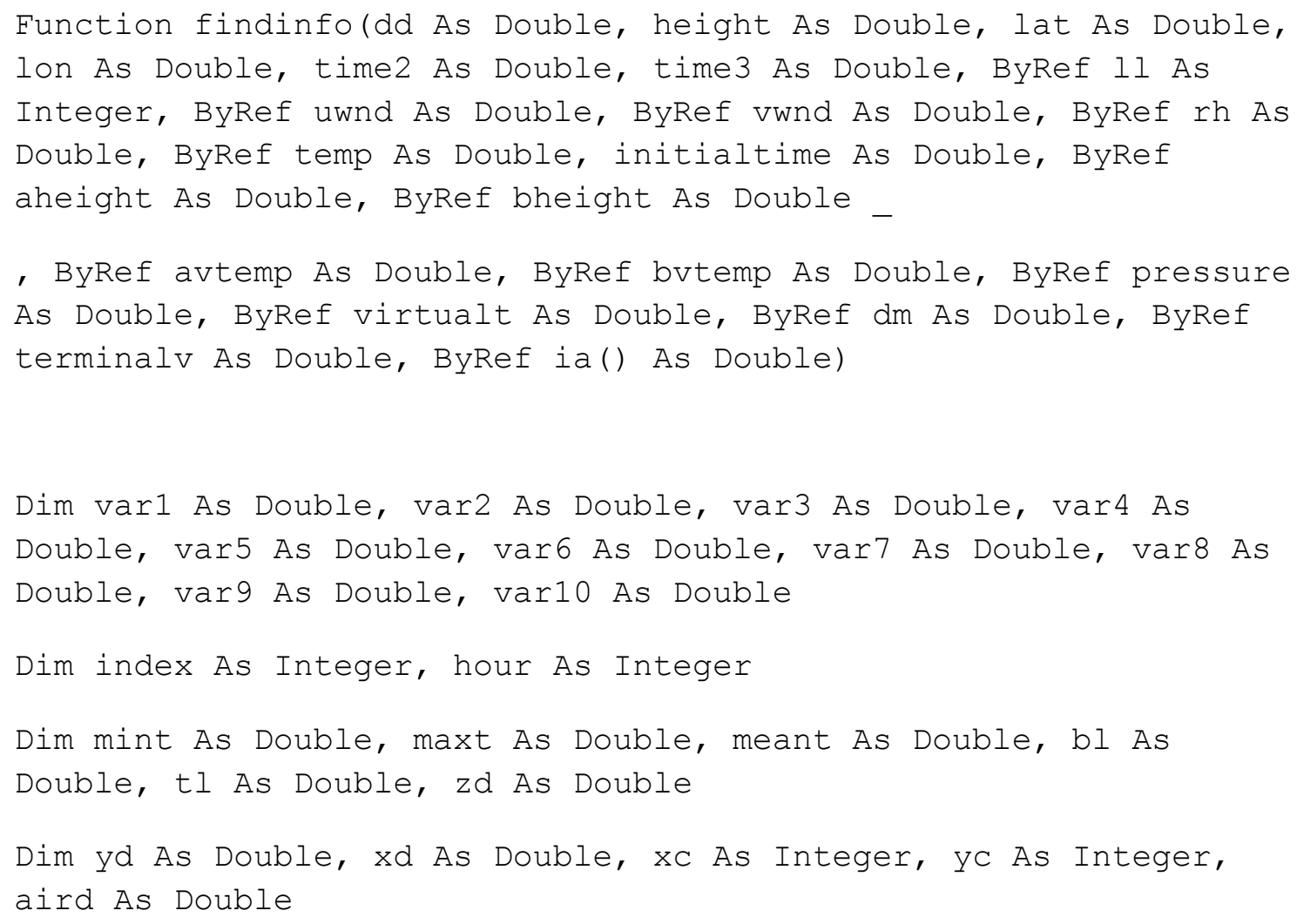




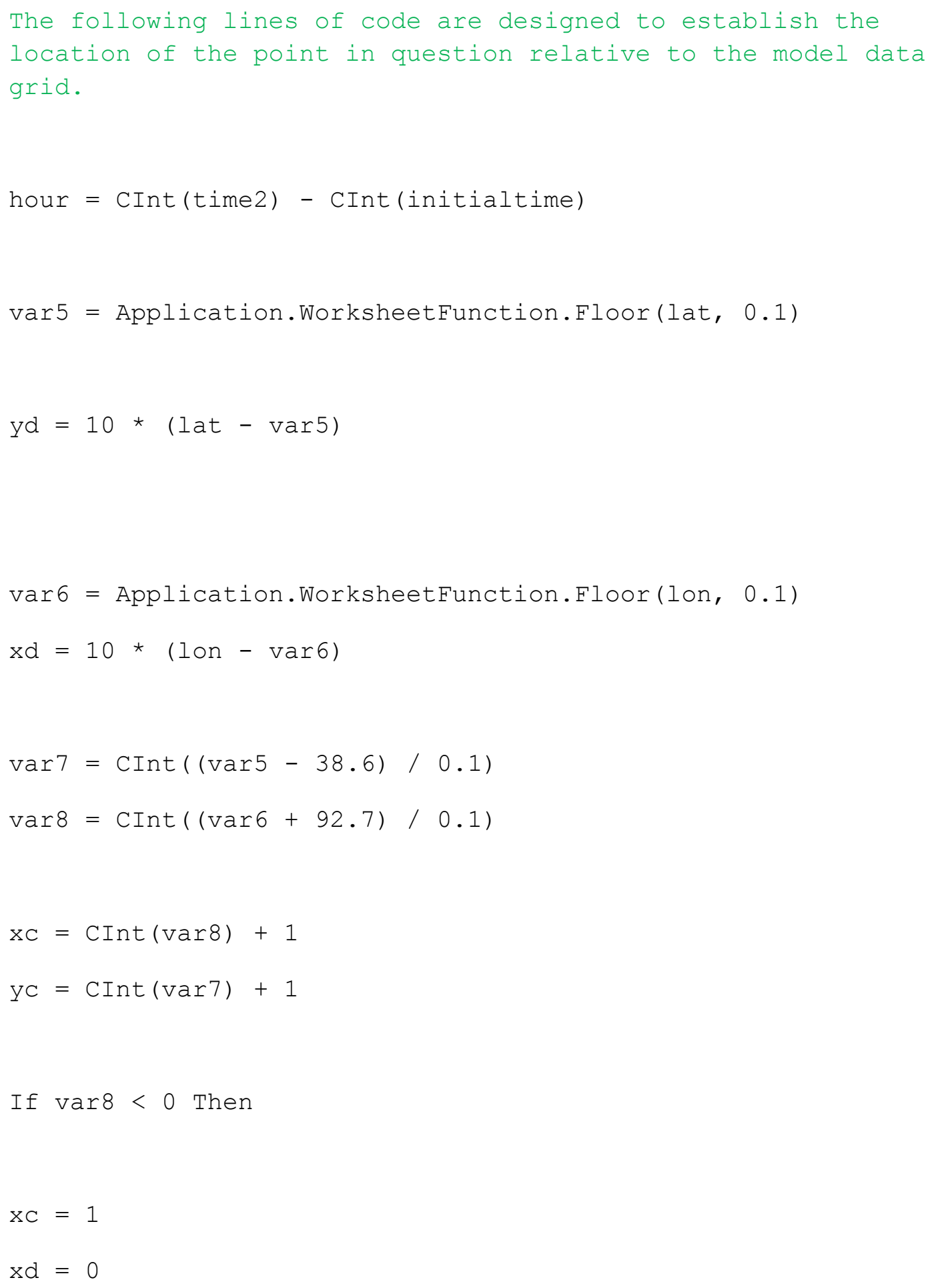




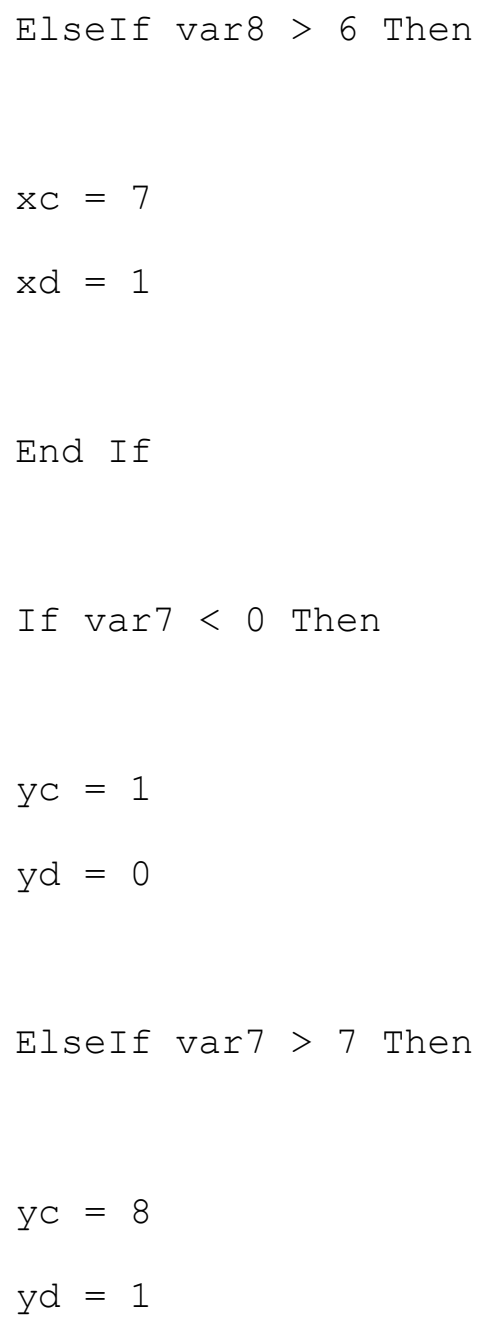

End If

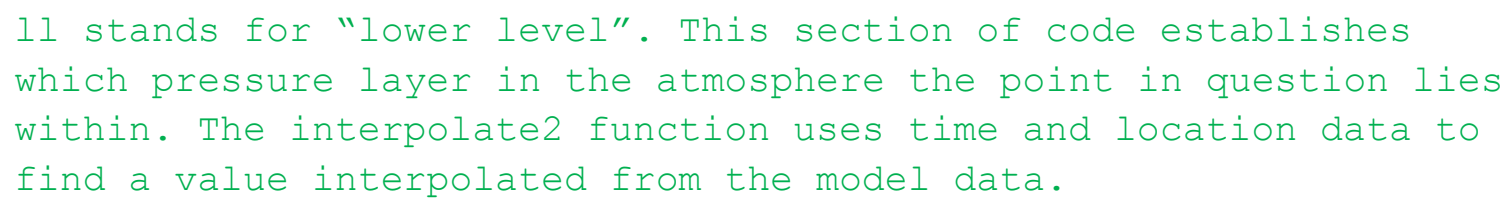




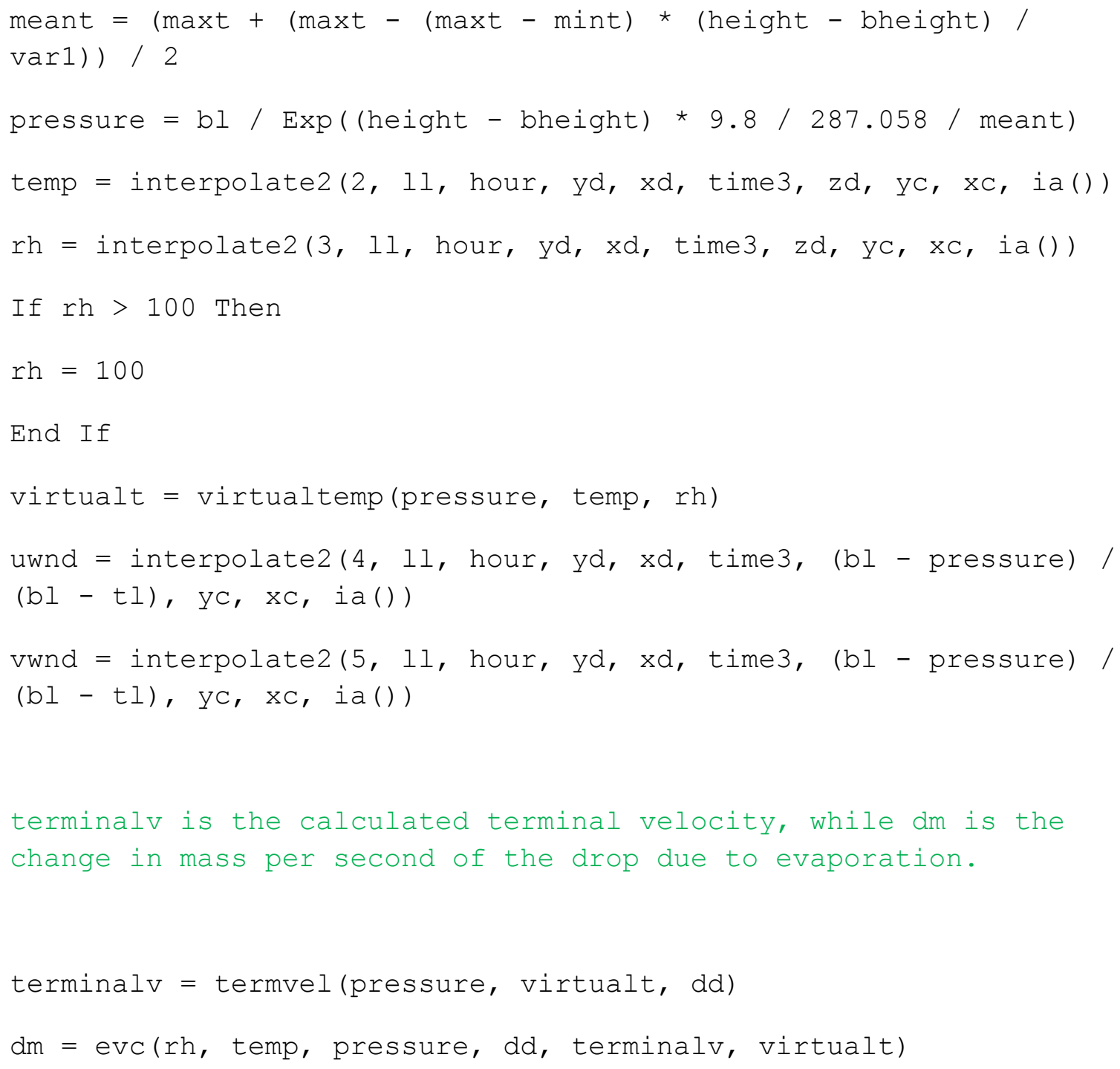

End Function 